\title{
Micrometeorological Data for Energy-Budget Studies Near Rogers Spring, Ash Meadows National Wildlife Refuge, Nye County, Nevada, 1994 \\ 1 ,
}

\section{U.S. GEOLOGICAL SURVEY}

Open-File Report 96-170
RECFIVED

MAY 021996

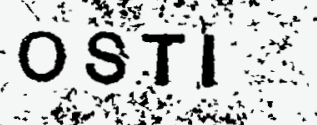

Prepared in cooperation with the U.S. DEPARTMENT OF ENERGY, OFFICE OF ENVIRONMENTAL RESTORATION AND WASTE MANAGEMENT, NEVADA OPERATIONS OFFICE, under Interagency Agreement DE-A108-91NV 11040

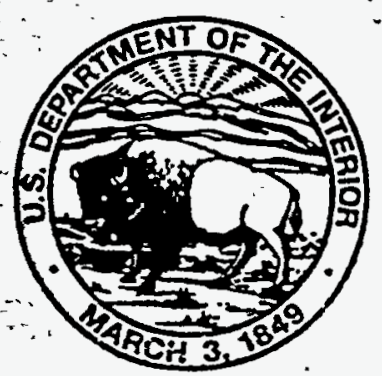




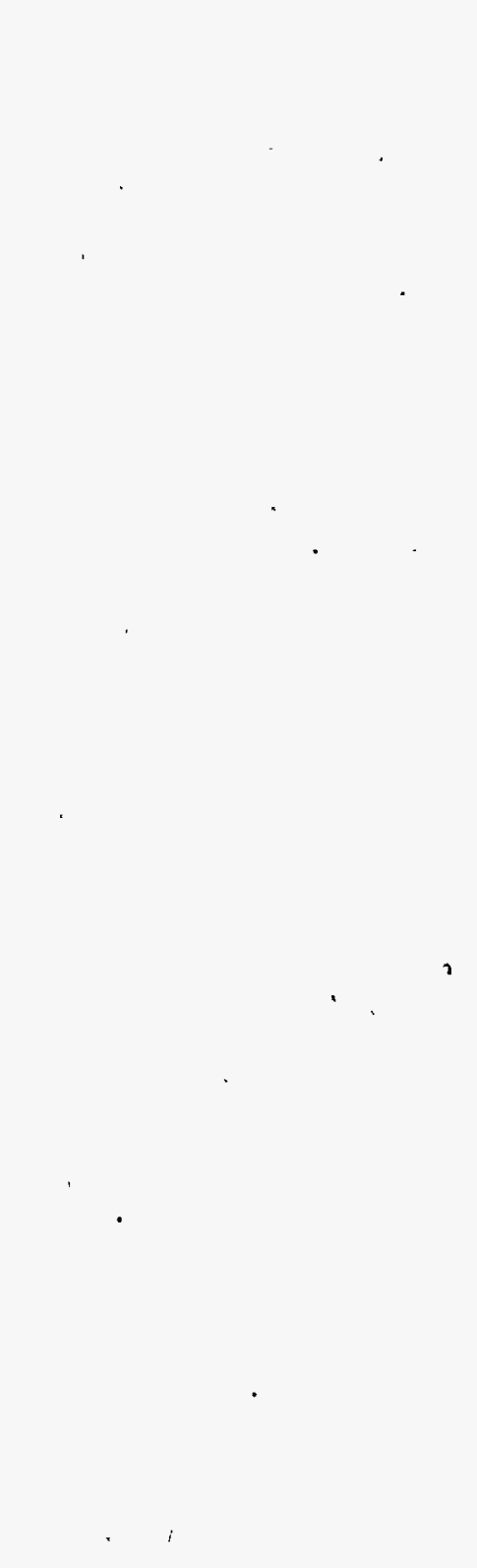




\title{
Micrometeorological Data for Energy-Budget Studies Near Rogers Spring, Ash Meadows National Wildlife Refuge, Nye County, Nevada, 1994
}

\author{
By William D. Nichols and Timothy R. Rapp
}

\section{U.S. GEOLOGICAL SURVEY}

Open-File Report 96-170

Prepared in cooperation with the U.S. DEPARTMENT OF ENERGY, OFFICE OF ENVIRONMENTAL RESTORATION AND WASTE MANAGEMENT NEVADA OPERATIONS OFFICE, under Interagency Agreement DE-Al08-91NV11040

\section{DISCLAIMER}

This report was prepared as an account of work sponsored by an agency of the United States Government. Neither the United States Government nor any agency thereof, nor any of their employees, makes any warranty, express or implied, or assumes any legal liability or responsibility for the accuracy, completeness, or usefulness of any information, apparatus, product, or process disclosed, or represents that its use would not infringe privately owned rights. Reference herein to any specific commercial product, process, or service by trade name, trademark, manufacturer, or otherwise does not necessarily constitute or imply its endorsement, recommendation, or favoring by the United States Government or any agency thereof. The views and opinions of authors expressed herein do not necessarily state or reflect those of the United States Government or any agency thereof.

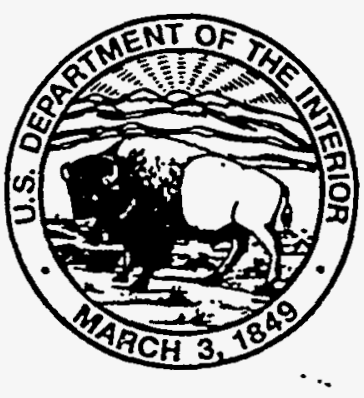

Carson City, Nevada 1996 


\title{
U.S. DEPARTMENT OF THE INTERIOR \\ BRUCE BABBITT, Secretary
}

\author{
U.S. GEOLOGICAL SURVEY \\ GORDON P. EATON, Director
}

Any use of trade names in this publication is for descriptive purposes

only and does not constitute endorsement by the U.S. Government

For additional information

write to:

District Chief

U.S. Geological Survey

333 West Nye Lane, Room 203

Carson City, NV 89706-0866
Copies of this report can be purchased from:

U.S. Geological Survey

Information Services

Box 25286, MS 517

Denver Federal Center

Denver, CO 80225-0046 


\section{CONTENTS}

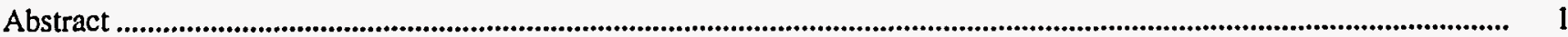

Introduction ...........................................................................................................................................................................

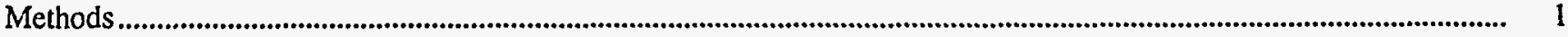

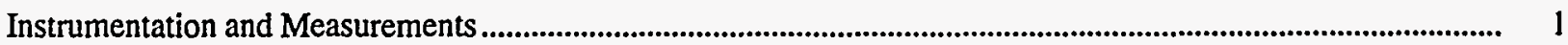

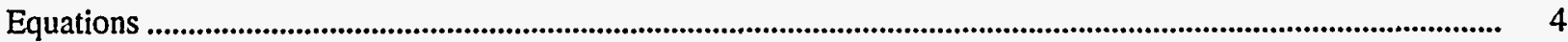

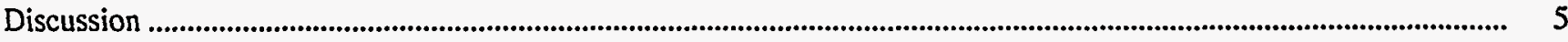

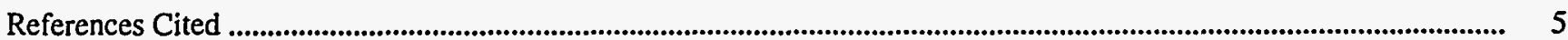

\section{DISKETTE}

(in pocket at back of report)

High-density, double-sided, soft-sectored, 3-1/2-inch diskette containing hourly value files

\section{FIGURES}

1. Map showing location of Death Valley ground-water flow system and three primary subbasins controlling ground-water flow at Nevada Test Site: Ash Meadows, Oasis Valley, and

Alkali Flat-Furnace Creek Ranch

2. Map showing location of Ash Meadows National Wildlife Refuge and Rogers Spring study sites

\section{TABLES}

1. Daily mean net radiation, air temperature, dew-point temperature, vapor pressure, soil heat flux, and soil temperature change, measured by energy-budget station at Rogers Spring Site 1, 1994

2. Daily mean, maximum, and minimum air and dew-point temperatures and vapor pressure, measured by energy-budget station at Rogers Spring Site 1, 1994

3. Daily mean, maximum, and minimum micrometeorological data measured by micromet station at

Rogers Spring Site 1, 1994 ............................................................................................
4. Daily mean net radiation, air temperature, dew-point temperature, vapor pressure, soil heat flux, and soil temperature change measured by energy-budget station at Rogers Spring Site.2, 1994

Daily mean, maximum, and minimum air and dew-point temperatures and vapor pressure measured by energy-budget station at Rogers Spring Site 2, 1994

6. Daily mean, maximum, and minimum micrometeorological data measured by micromet station at Rogers Spring Site 2, 1994 


\begin{tabular}{|c|c|c|}
\hline Multiply & By & To obtain \\
\hline \multicolumn{3}{|l|}{ Area } \\
\hline square hectometer $\left(\mathrm{hm}^{2}\right)$ & 2.471 & acre \\
\hline kilogram per cubic meter $\left(\mathrm{kg} / \mathrm{m}^{3}\right)$ & $6.2428 \times 10^{-2}$ & pound per cubic foot \\
\hline \multicolumn{3}{|l|}{ Energy and Area Time } \\
\hline watt per square meter $\left(\mathrm{W} / \mathrm{m}^{2}\right)$ & $5.2895 \times 10^{-3}$ & $\begin{array}{l}\text { British thermal unit per square } \\
\text { foot per minute }\end{array}$ \\
\hline \multicolumn{3}{|l|}{ Energy and Mass } \\
\hline \multicolumn{3}{|r|}{ - } \\
\hline $\begin{array}{r}\text { centimeter }(\mathrm{cm}) \\
\text { micrometer }(\mu \mathrm{m})\end{array}$ & $\begin{array}{l}2.54 \\
0.3937\end{array}$ & $\begin{array}{l}\text { inch } \\
\text { mil }\end{array}$ \\
\hline $\operatorname{meter}(\mathrm{m})$ & 3.281 & foot \\
\hline kilometer (km) & 0.621 & mile \\
\hline \multicolumn{3}{|l|}{ Specific heat capacity } \\
\hline joules per kilogram per degree kelvin $\left[\mathrm{J} /\left(\mathrm{kg}^{\circ} \mathrm{K}\right)\right]$ & $2.388 \times 10^{-4}$ & $\begin{array}{l}\text { British thermal unit per pound } \\
\text { per degree Fahrenheit }\end{array}$ \\
\hline \multicolumn{3}{|r|}{ 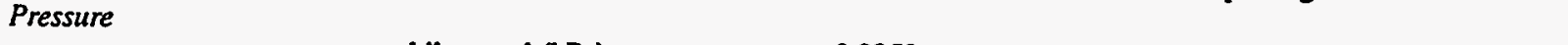 } \\
\hline & 10 & $\begin{array}{l}\text { pound per square inch } \\
\text { millibar }\end{array}$ \\
\hline \multicolumn{3}{|l|}{ Temperature } \\
\hline degree Celsius $\left({ }^{\circ} \mathrm{C}\right)$ & $1.8^{\circ} \mathrm{C}+32$ & degree Fahrenheit \\
\hline degree kelvin $\left({ }^{\circ} \mathrm{K}\right)$ & $\left({ }^{\circ} \mathrm{K}-273.15\right) 1.8+32$ & degree Fahrenheit \\
\hline \multicolumn{3}{|l|}{ Velocity or Rate } \\
\hline meter per second $(\mathrm{m} / \mathrm{s})$ & 2.237 & mile per hour \\
\hline
\end{tabular}

Sea level: In this report, "sea level" refers to the National Geodetic Vertical Datum of 1929 (NGVD of 1929, formerly called "Sea-Level Datum of 1929"), which is derived from a general adjustment of the first-order leveling networks of the United States and Canada.

\section{LIST OF SYMBOLS}

$C_{\mathrm{s}} \quad$ Specific heat capacity of dry soil, $\mathrm{J} / \mathrm{kg}^{\circ} \mathrm{K}$

$C_{\mathrm{w}} \quad$ Specific heat capacity of water, $\mathrm{J} / \mathrm{kg}^{\circ} \mathrm{K}$

$d \quad$ Depth of heat flux transducer, $m$

$D_{\mathrm{b}} \quad$ Bulk density of soil, $\mathrm{kg} / \mathrm{m}^{3}$

$e_{1,2} \quad$ Vapor pressure above canopy reference height, $\mathrm{kPa}$

$G$ Soil heat flux, $\mathrm{W} / \mathrm{m}^{2}$

$H$ Sensible heat above the canopy, $W / \mathrm{m}^{2}$

$L E \quad$ Latent heat flux above the canopy, $W / \mathrm{m}^{2}$

$R n \quad$ Net radiation on soil and canopy, $W / \mathrm{m}^{2}$

$t$ Time sampling interval, $s$

$T_{1,2} \quad$ Air temperature at above-canopy reference height, ${ }^{\circ} \mathrm{C}$

$T^{\mathrm{c}} \quad$ Canopy temperature, ${ }^{\circ} \mathrm{C}$

$T_{\mathrm{s}} \quad$ Soil surface temperature, ${ }^{\circ} \mathrm{C}$

$u \quad$ Windspeed at above-canopy reference height, $\mathrm{m} / \mathrm{s}$

$W \quad$ Soil-water content, gravimetric

$\beta \quad$ Bowen ratio, dimensionless

$\Delta e \quad$ Vapor pressure difference, $\mathrm{kPa}$

$\Delta T \quad$ Air temperature difference, ${ }^{\circ} \mathrm{C}$

$\Delta T_{\mathrm{s}} \quad$ Difference in soil temperature over time, ${ }^{\circ} \mathrm{C}$

$\gamma \quad$ Psychrometric constant, $\mathrm{kPa}{ }^{\circ} \mathrm{K}$ 


\section{Micrometeorological Data for Energy-Budget Studies Near Rogers Spring, Ash Meadows National Wildlife Refuge, Nye County, Nevada, 1994}

\author{
By William D. Nichols and Timothy R. Rapp
}

\section{ABSTRACT}

Micrometeorological data were collected at two sites near Rogers Spring in the Ash Meadows National Wildlife Refuge for use in energybudget studies beginning in 1994. The data collected at each site included net radiation, air temperature at two heights, dew-point temperature at two heights, windspeed at two heights, soil heat flux, and soil temperature in the interval between the land surface and the buried heat-flux plates.

\section{INTRODUCTION}

The Ash Meadows National Wildlife Refuge is in the Mojave Desert in south-central Nye County, Nev., near the border with California (fig. 1). The refuge includes about $8,840 \mathrm{hm}^{2}$ of desert uplands and spring-fed oases and is about $65 \mathrm{~km}$ east of the Death Valley National Monument headquarters at Furnace Creek, Calif., and about $145 \mathrm{~km}$ northwest of Las Vegas, Nev. Altitudes range from 600 to $700 \mathrm{~m}$ above sea level. Springs in the Ash Meadows area are points of ground-water discharge from the Ash Meadows subbasin of the Death Valley ground-water flow system (fig. 1; Waddell and others, 1984; Harrill and others, 1988).

Micrometeorological data collection was begun in 1994 at two sites near Rogers Spring in the Ash Meadows National Wildlife Refuge (fig. 2) for use in energy-budget studies. The energy budget is solved to estimate the amount of ground-water discharged by evapotranspiration, which is related to the discharge of springs throughout the Ash Meadows area. These data were collected by the U.S. Geological Survey, in cooperation with the U.S. Department of Energy Environmental Restoration Program.
The purpose of this report is to describe the instrumentation and procedures used for data collection and to present and describe the data collected in 1994 that are needed to solve the energy-budget equation.

Both sites near Rogers Spring are in the NWl/4 sec. 15, T. 17 S., R. 50 E. Rogers Spring Site 1 is at lat $36^{\circ} 28^{\prime} 56^{\prime \prime} \mathrm{N}$., long $116^{\circ} 19^{\prime} 56^{\prime \prime} \mathrm{W}$, at an altitude of $687.4 \mathrm{~m}$. The land surface at this site is about half bare soil and half scattered patches of dense saltgrass. The saltgrass is about $18 \mathrm{~cm}$ high. The ground-water level at this site was about $0.75 \mathrm{~m}$ below land surface on May 15, 1994. By September 11, 1994, it was $2.19 \mathrm{~m}$ below land surface. Rogers Spring Site 2 is $245 \mathrm{~m}$ northeast of Site 1 at $36^{\circ} 28^{\prime} 55^{\prime \prime}$ N., $116^{\circ} 20^{\prime} 06^{\prime \prime}$ W. and is at an aititude of $686.6 \mathrm{~m}$. The site is in a saltgrass marsh with only about 5 percent exposed bare soil. The saltgrass is about $25 \mathrm{~cm}$ high. Ground water was at land surface in January 1994, about $0.1 \mathrm{~m}$ below land surface on May 15, and about $2.25 \mathrm{~m}$ below land surface by September 11.

\section{METHODS}

\section{Instrumentation and Measurements}

Two separate and complete instrumentation stations, a micromet station and an energy-budget station, were in operation at each site for part of the year, with one station in operation for the entire year at each site. A micromet station measured net radiation, air temperature at two heights, humidity at one height, and windspeed at two heights (tables 3 and 6). An energybudget station, which was in operation beginning March 16, measured net radiation, air temperature.and dew-point temperature at two heights, soil heat flux, and changes in soil temperature (tables $1,2,4$, and 5). 


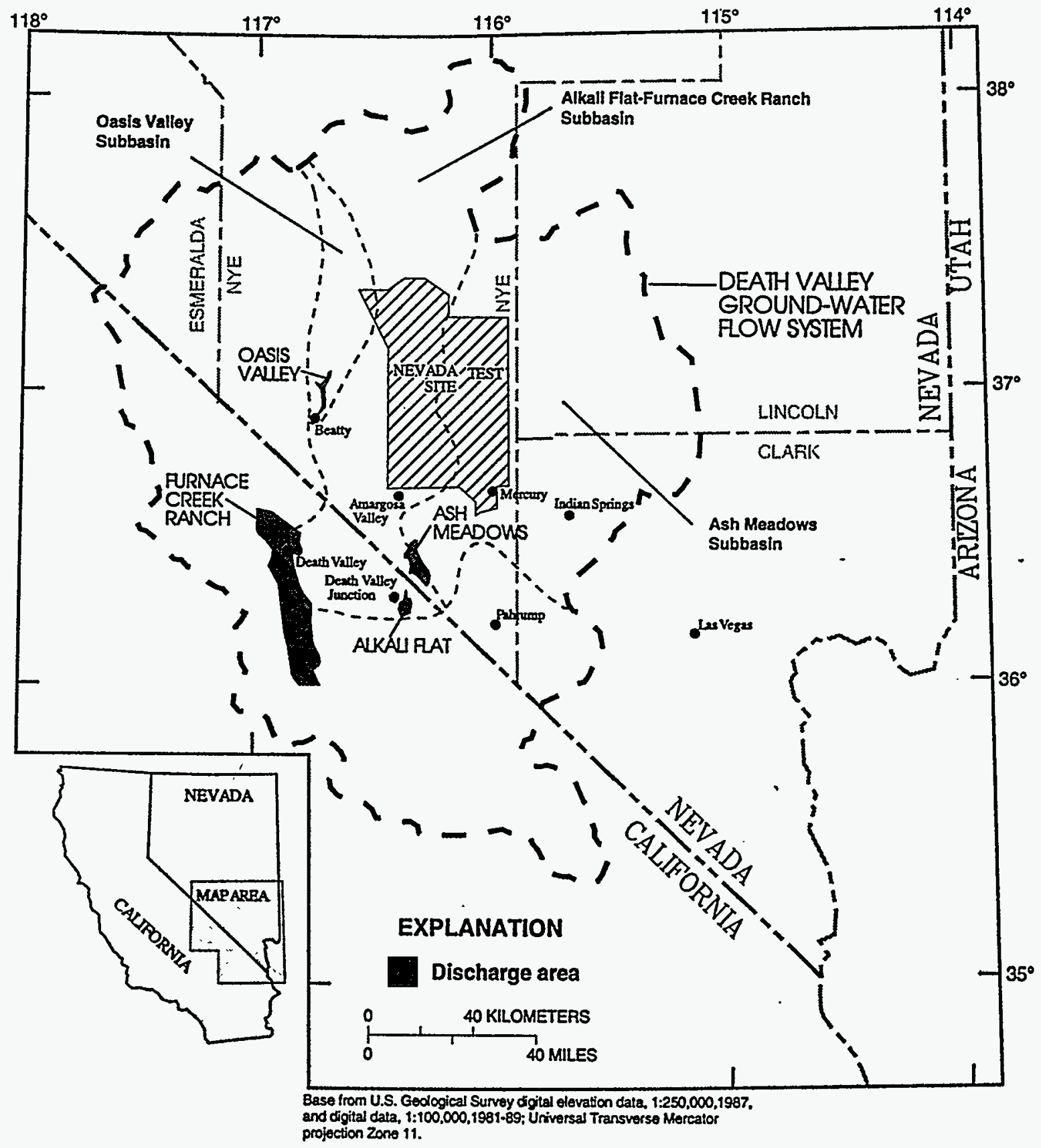

Figure 1. Location of Death Valley ground-water flow system and three primary subbasins controlling groundwater flow at Nevada Test Site (Randell J. Laczniak, U.S. Geological Survey, written commun., 1996): Ash Meadows, Oasis Valley, and Alkali Flat-Furnace Creek Ranch. Major discharge areas are Ash Meadows, Oasis Valley, Alkali Flat, and Furnace Creek Ranch (Death Valley). 


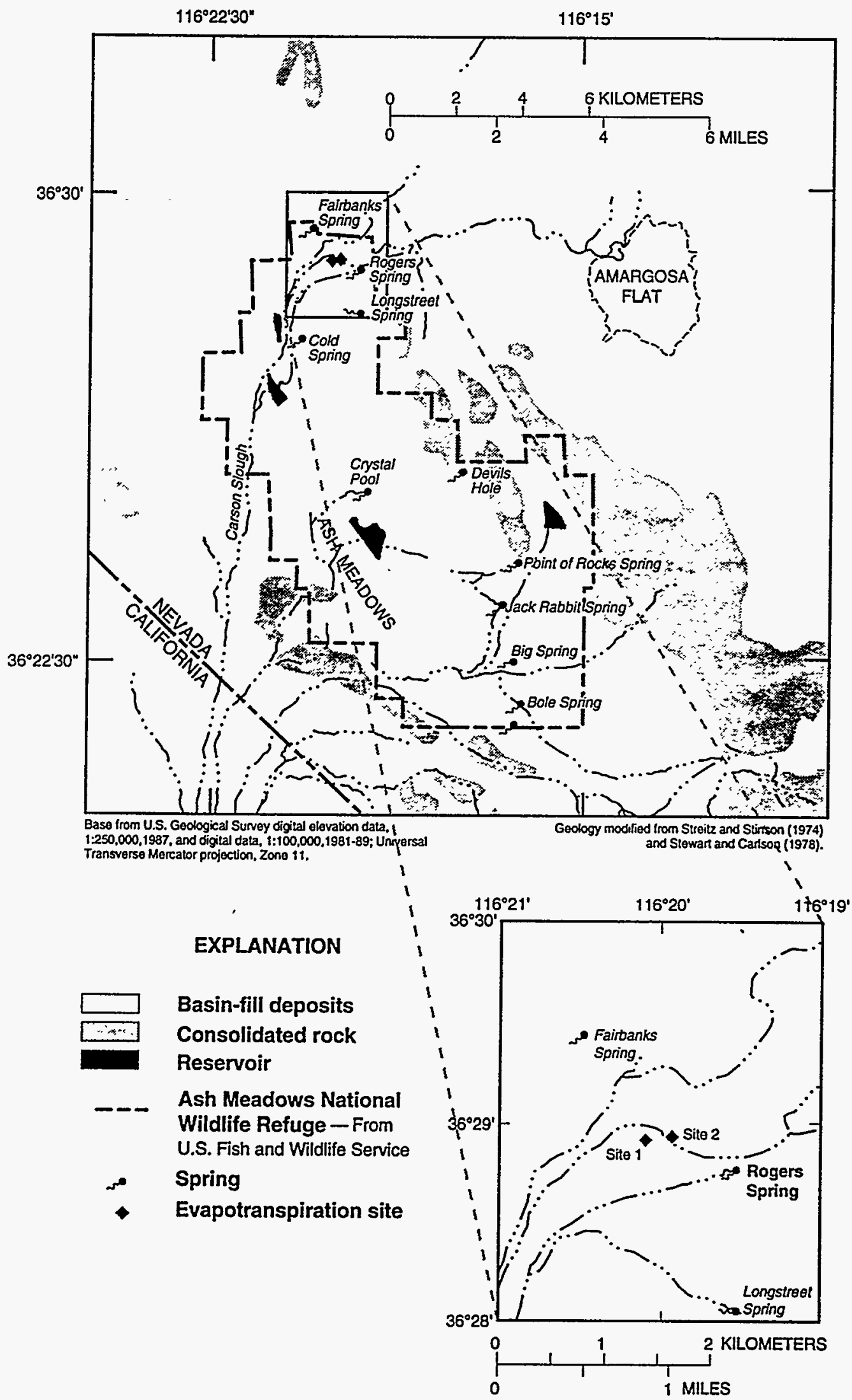

Figure 2. Location of Ash Meadows National Wildlife Refuge and Rogers Spring study sites. 
The latter station was operated only during daylight hours through winter because of night-time freezing temperatures that interfere with the measurement of dew-point temperature. Minor differences occur in some measured variables common to the two stations because of differences in instruments used or atmospheric differences in the vicinity of the station even though the stations are within $5 \mathrm{~m}$ of one another.

Net radiation was measured at both instrument stations at each location with a Radiation Energy Balances Systems net radiometer model Q6.

Vapor-pressure gradients were determined by the energy-budget station at each site by measuring dew-point temperatures at two heights using a Campbell Scientific, Inc. (CSI) single-cooled-mirror hygrometer. The measuring heights at Site 1 were $0.5 \mathrm{~m}$ and $1.5 \mathrm{~m}$ above the top of the canopy and at Site 2 were $0.7 \mathrm{~m}$ and $1.7 \mathrm{~m}$ above the top of the canopy. Air is drawn alternately through intakes at each height and routed to the cooled mirror; a single pump aspirates the system. Problems of measuring vapor pressures that typically are associated with systematic sensor error are avoided by this system by using a single sensor. However, operating limitations of the cooled mirror under conditions of high temperature and low humidity can result in invalid dew-point temperatures and consequently invalid vapor pressures. These conditions occur when ambient temperature approaches $35^{\circ} \mathrm{C}$ and humidity drops below 10 percent.

Mirror contamination may occur in areas of saltgrass and salt marsh. When this happens, measured dew-point temperatures, and hence vapor pressures, are too high, but differences in dew-point temperature measured at the two heights may be approximately correct. Mirror contamination was a problem at Site 1 beginning in late July and continuing into September. More frequent servicing of the equipment minimized the problem after mid-August. No attempt was made to correct dew-point temperature measurements or calculated vapor pressures because of this problem. Air temperature was measured at the same two heights using 76- $\mu \mathrm{m}$ diameter unshielded, non-aspirated chromel-constantan thermocouples.

Soil heat flux was measured with two Radiation Energy Balance Systems heat-flow transducers buried at $0.05 \mathrm{~m}$. Change in soil temperature in the soil layer above each transducer was measured by four thermocouples wired in parallel to provide a spatially averaged soil temperature. Two of the four thermocouples were placed above each transducer, one at a depth of about $0.01 \mathrm{~m}$ and one at a depth of about $0.03 \mathrm{~m}$. The change in soil temperature measured by the thermocouple, together with periodically measured soil-water content and soil bulk density and an estimated value for the specific heat of dry soil, were used to calculate changes in soil heat storage in the interval above the flux transducers. This heat storage was added to the flux measured by the transducer.

Soil heat flux can be a significant component of the energy budget in sparse-canopy rangelands. Both sets of sensors were placed in full sun locations to measure the maximum soil heat flux throughout the day. This maximum value was modified for the area of bare soil at each study site.

Air and dew-point temperatures were sampled at 1-s intervals and averaged for 20 -min periods. These data were collected from March 16, 1994, through the end of the calendar year. Radiation, soil heat flux, and soil temperatures were sampled at 10-s intervals and averaged for $1-\mathrm{hr}$ periods from January 1 to March 15 and for 20-min periods beginning on March 16. Data were collected with CSI 21X microloggers.

Data collected at the micromet station at each site included air temperature, humidity, and windspeed. Air temperature and humidity were measured with a Rotronics temperature-humidity sensor mounted at $0.7 \mathrm{~m}$ above the soil surface at Site 1 and $1.25 \mathrm{~m}$ above the soil surface at Site 2 .

Windspeed was measured using R.M. Young, Inc., photo-chopper anemometers with a threshold of $0.2 \mathrm{~m} / \mathrm{s}$. Windspeed measurements were made at the same heights as those of air and dew-point temperature and relative humidity. Air temperature, humidity, and windspeed were sampled at $10-s$ intervals. Data were averaged for $1-\mathrm{hr}$ intervals from January 1 to March 15 and for 20-min intervals from March 16 to December 31 . The 20 -min data were averaged for $1-\mathrm{hr}$ intervals and hourly data were averaged for daily values. Data were collected with CSI $21 X$ microloggers.

\section{Equations}

Values of net radiation, air-temperature gradient, vapor-pressure gradient, and soil-heat flux are used to solve the energy budget, given by (symbols used in the following equations are given in the table at the front of the report.)

$$
R n=L E+H+G \quad .
$$

using the Bowen-ratio method (Tanner, 1960). 
Net radiation, $R n$, and soil heat flux, $G$, are measured. Latent heat flux, $L E$, and sensible heat flux, $H$, are not measured and equation 1 cannot be solved directly; the Bowen-ratio method (Bowen, 1926) is used. The Bowen ratio is the ratio of sensible to latent heat fluxes and is given by

$$
\beta=\frac{H}{L E}=\gamma \frac{\Delta T}{\Delta e}=\gamma \frac{T_{1}-T_{2}}{e_{1}-e_{2}} .
$$

Latent heat flux is calculated by substituting equation 2 in equation 1 , so that

$$
L E=\frac{R_{n}-G}{1+\beta} .
$$

Then, sensible heat flux is given by

$$
H=\beta L E .
$$

Measured soil heat fiux must be corrected for heat stored in the soil interval above the buried heat flux transducer. This correction is given by

$$
S_{s}=\frac{\Delta T_{s}}{t} d D_{b}\left(C_{s}+W C_{w}\right)
$$

These equations are not solved in this report, but are included to demonstrate one use of the data collected.

\section{DISCUSSION}

Data used to solve the Bowen ratio-energy budget equation were collected during 1994 beginning on March 16. Daily mean values of these data are given in table 1 for site 1 and table 4 for site 2 . Daily values are not appropriate for calculating daily energy budgets; hourly averaged values should be used and the resulting values then averaged to obtain daily components of the energy budget. Hourly averaged values for each site are in files on the diskette included with this report. The disk is in IBM-compatible format for DOS-based computers. A short text file, named readme.txt, describes the variables included in each data file for each site. These data include day, time, net radiation, air temperature, vapor pressure, soil heat flux, change in soil temperature, and windspeed.

Daily mean, maximum, and minimum values for air temperature, dew-point temperature, and vapor pressure at the two sites are given in tables 2 and 5 .

Micrometeorological data collected by the micromet station at each site are in tables 3 and 6 .
These tables give daily mean and maximum values of net radiation, and daily mean, maximum, and minimum values of air temperature, humidity, and windspeed. Hourly averaged values of these data are in files on the diskette included with this report.

Differences in net radiation measured by the net radiometers at Site 1 reflect the differences in land cover beneath each net radiometer. The net radiometer on the permanent station views more bare soil than does the net radiometer on the temporary station. Consequently, the permanent-station net radiometer measures more reflected short-wave radiation and more emitted long-wave radiation than does the other radiometer. The resulting net radiation measured at the permanent station is lower than that measured at the temporary station.

Mean daily air temperatures measured at Site 1 are slightly higher at the permanent station. These temperatures were measured with a Rotronics temperature-humidity sensor; temperatures at the temporary station were measured with fine-wire thermocouples. Typically, the Rotronics measures from 0.5 to $1^{\circ} \mathrm{C}$ higher than the thermocouples, but this depends in part on the accuracy of the thermocouple reference temperature in the CSI 21X data logger. Additionally, the Rotronics sensor is in a nonaspirated radiation shield and is affected by thermal loading during daylight hours.

\section{REFERENCES CITED}

Bowen, I.S., 1926, The ratio of heat loss by conduction and by evaporation from any water surface: Physics Review, v. 27, p. 779-787. .

Harrill, J.R., Gates, J.S., and Thomas, J.M., 1988, Major ground-water flow systems in the Great Basin region of Nevada, Utah, and adjacent states: U.S. Geological Survey Hydrologic Investigations Atlas HA-694-C, 2 sheets, scale $1: 1,000,000$.

Stewart, J.H., and Carlson, J.E., 1978, Geologic map of Nevada: U.S. Geological Survey, 1:500,000 scale.

Streitz, Robert, and Stinson, M.C., 1974, Geologic map of California, Death Valley Sheet: California Division of Mines and Geology, scale 1:250,000.

Tanner, C.B., 1960, Energy balance approach to evapotranspiration from crops: Soil Science Society of America, Proceedings, v. 24, p. 1-9.

Waddell, R.K., Robinson, J.H., and Blankennagel, R.K., 1984, Hydrology of Yucca Mountain and vicinity, Nevada-California-Investigative results through mid1983: U.S. Geological Survey Water Resources Investigations Report 84-4267, 72 p. 
Table 1. Daily mean net radiation, air temperature, dew-point temperature, vapor pressure, soil heat flux, and soil temperature change, measured by energy-budget station at Rogers Spring Site 1, 1994

\begin{tabular}{|c|c|c|c|c|c|c|c|c|c|c|}
\hline \multirow[t]{2}{*}{$\begin{array}{l}\text { Calendar } \\
\text { day }\end{array}$} & \multirow{2}{*}{$\begin{array}{c}\text { Net } \\
\text { radiation, } \\
\text { in watts } \\
\text { per square } \\
\text { meter }\end{array}$} & \multicolumn{2}{|c|}{$\begin{array}{l}\text { Air temperature, } \\
\text { in degrees Celsius }\end{array}$} & \multicolumn{2}{|c|}{$\begin{array}{l}\text { Dewpoint temperature, } \\
\text { in degrees Celsius }\end{array}$} & \multicolumn{2}{|c|}{$\begin{array}{l}\text { Vapor pressure, } \\
\text { in kilopascals }\end{array}$} & \multicolumn{2}{|c|}{$\begin{array}{l}\text { Soil-heat flux, } \\
\text { in watts per } \\
\text { square meter }\end{array}$} & \multirow{2}{*}{$\begin{array}{c}\text { Difference in soil } \\
\text { temperature over } \\
\text { time, in degrees } \\
\text { Celsius }\end{array}$} \\
\hline & & 0.7 meter & 1.7 meters & 0.7 meter & 1.7 meters & 0.7 meter & 1.7 meters & Plate 1 & Plate 2 & \\
\hline $\begin{array}{l}75 \\
76 \\
77 \\
78 \\
79\end{array}$ & $\begin{array}{l}58.8 \\
72.4 \\
80.5 \\
54.1 \\
92.0\end{array}$ & $\begin{array}{l}21.41 \\
18.05 \\
18.58 \\
14.63 \\
11.87\end{array}$ & $\begin{array}{l}21.47 \\
18.32 \\
18.51 \\
14.63 \\
12.23\end{array}$ & $\begin{array}{r}-6.75 \\
-6.97 \\
-3.16 \\
.75 \\
.24\end{array}$ & $\begin{array}{r}-6.91 \\
-6.10 \\
-1.38 \\
3.43 \\
1.16\end{array}$ & $\begin{array}{r}0.4043 \\
.3715 \\
.4939 \\
.6556 \\
.6547\end{array}$ & $\begin{array}{r}0.3961 \\
.3889 \\
.5600 \\
.7880 \\
.6798\end{array}$ & $\begin{array}{r}8.3 \\
8.4 \\
8.2 \\
2.2 \\
-1.2\end{array}$ & $\begin{array}{r}1.1 \\
.4 \\
.8 \\
-1.4 \\
-3.2\end{array}$ & $\begin{array}{r}0.008 \\
.004 \\
.008 \\
-.009 \\
-.011\end{array}$ \\
\hline $\begin{array}{l}80 \\
81 \\
82 \\
83 \\
84\end{array}$ & $\begin{array}{r}85.9 \\
77.7 \\
85.7 \\
65.9 \\
102.2\end{array}$ & $\begin{array}{r}14.10 \\
12.45 \\
9.23 \\
11.86 \\
8.82\end{array}$ & $\begin{array}{r}14.25 \\
12.64 \\
9.31 \\
11.97 \\
8.75\end{array}$ & $\begin{array}{l}-.08 \\
-4.05 \\
-9.64 \\
-5.47 \\
-3.64\end{array}$ & $\begin{array}{r}1.02 \\
-4.47 \\
-10.54 \\
-3.80 \\
-1.10\end{array}$ & $\begin{array}{l}.6440 \\
.4839 \\
.3081 \\
.4168 \\
.4779\end{array}$ & $\begin{array}{l}.6760 \\
.4735 \\
.2772 \\
.4661 \\
.5701\end{array}$ & $\begin{array}{r}2.0 \\
-7.9 \\
-6.4 \\
-.6 \\
-.9\end{array}$ & $\begin{array}{l}-1.6 \\
-5.8 \\
-5.3 \\
-2.6 \\
-1.8\end{array}$ & $\begin{array}{r}.021 \\
-.047 \\
.023 \\
-.002 \\
-.015\end{array}$ \\
\hline $\begin{array}{l}85 \\
86 \\
87 \\
88 \\
89\end{array}$ & $\begin{array}{l}95.9 \\
90.2 \\
84.9 \\
95.8 \\
95.9\end{array}$ & $\begin{array}{l}11.28 \\
14.00 \\
14.63 \\
15.03 \\
17.22\end{array}$ & $\begin{array}{l}11.34 \\
14.57 \\
14.92 \\
15.57 \\
17.66\end{array}$ & $\begin{array}{l}-2.71 \\
-6.05 \\
-6.67 \\
-6.10 \\
-4.84\end{array}$ & $\begin{array}{l}-2.37 \\
-7.24 \\
-6.81 \\
-5.65 \\
-4.96\end{array}$ & $\begin{array}{l}.5273 \\
.4058 \\
.3947 \\
.4109 \\
.4442\end{array}$ & $\begin{array}{l}.5199 \\
.3572 \\
.3706 \\
.4048 \\
.4248\end{array}$ & $\begin{array}{r}-4.5 \\
-1.3 \\
3.4 \\
5.6 \\
7.7\end{array}$ & $\begin{array}{r}-4.2 \\
-2.5 \\
-1.4 \\
-.2 \\
1.2\end{array}$ & $\begin{array}{r}.016 \\
-.005 \\
.020 \\
.012 \\
.002\end{array}$ \\
\hline $\begin{array}{l}90 \\
91 \\
92 \\
93 \\
94\end{array}$ & $\begin{array}{r}101.4 \\
103.3 \\
88.4 \\
53.6 \\
95.4\end{array}$ & $\begin{array}{l}16.52 \\
15.19 \\
17.03 \\
18.86 \\
14.84\end{array}$ & $\begin{array}{l}17.19 \\
15.77 \\
17.50 \\
18.90 \\
14.83\end{array}$ & $\begin{array}{l}-5.15 \\
-6.52 \\
-6.08 \\
-2.94 \\
-5.75\end{array}$ & $\begin{array}{l}-4.69 \\
-6.19 \\
-5.95 \\
-1.59 \\
-6.35\end{array}$ & $\begin{array}{l}.4462 \\
.3971 \\
.4078 \\
.5010 \\
.4181\end{array}$ & $\begin{array}{l}.4408 \\
.3881 \\
.3945 \\
.5478 \\
.3981\end{array}$ & $\begin{array}{r}4.4 \\
-4.4 \\
4.6 \\
1.5 \\
-3.9\end{array}$ & $\begin{array}{r}-.6 \\
-4.7 \\
-.7 \\
-2.2 \\
-4.8\end{array}$ & $\begin{array}{r}-.006 \\
-.004 \\
.014 \\
-.006 \\
-.014\end{array}$ \\
\hline $\begin{array}{l}95 \\
96 \\
97 \\
98 \\
99\end{array}$ & $\begin{array}{r}99.5 \\
66.3 \\
104.9 \\
76.8 \\
81.6\end{array}$ & $\begin{array}{l}12.99 \\
14.70 \\
14.90 \\
14.85 \\
11.50\end{array}$ & $\begin{array}{l}13.25 \\
14.85 \\
15.19 \\
14.73 \\
11.56\end{array}$ & $\begin{array}{l}-9.38 \\
-7.10 \\
-5.43 \\
-4.19 \\
-4.00\end{array}$ & $\begin{array}{l}-9.13 \\
-5.92 \\
-4.44 \\
-2.22 \\
-2.05\end{array}$ & $\begin{array}{l}.3155 \\
.3751 \\
.4205 \\
.4572 \\
.4687\end{array}$ & $\begin{array}{l}.3093 \\
.4046 \\
.4415 \\
.5210 \\
.5302\end{array}$ & $\begin{array}{r}-2.2 \\
.1 \\
3.0 \\
3.2 \\
-2.2\end{array}$ & $\begin{array}{l}-3.6 \\
-1.5 \\
-1.4 \\
-1.7 \\
-3.7\end{array}$ & $\begin{array}{r}.007 \\
.005 \\
.011 \\
-.006 \\
-.015\end{array}$ \\
\hline $\begin{array}{l}100 \\
101 \\
102 \\
103 \\
104\end{array}$ & $\begin{array}{r}99.0 \\
112.8 \\
105.9 \\
112.1 \\
93.8\end{array}$ & $\begin{array}{l}13.84 \\
14.26 \\
15.54 \\
17.36 \\
18.62\end{array}$ & $\begin{array}{l}13.74 \\
14.80 \\
16.11 \\
17.83 \\
19.21\end{array}$ & $\begin{array}{l}-3.81 \\
-3.76 \\
-3.86 \\
-5.07 \\
-3.61\end{array}$ & $\begin{array}{l}-4.12 \\
-2.77 \\
-3.99 \\
-5.65 \\
-2.84\end{array}$ & $\begin{array}{l}.4736 \\
.4918 \\
.4968 \\
.4513 \\
.4996\end{array}$ & $\begin{array}{l}.4527 \\
.5046 \\
.4631 \\
.4058 \\
.4999\end{array}$ & $\begin{array}{r}-5.8 \\
1.9 \\
5.7 \\
8.0 \\
6.4\end{array}$ & $\begin{array}{r}-5.2 \\
-1.8 \\
-.1 \\
1.0 \\
.5\end{array}$ & $\begin{array}{l}.002 \\
.006 \\
.012 \\
.008 \\
.010\end{array}$ \\
\hline
\end{tabular}


Table 1. Daily mean net radiation, air temperature, dew-point temperature, vapor pressure, soil heat flux, and soil temperature change, measured by energy-budget station at Rogers Spring Site 1,1994-Continued

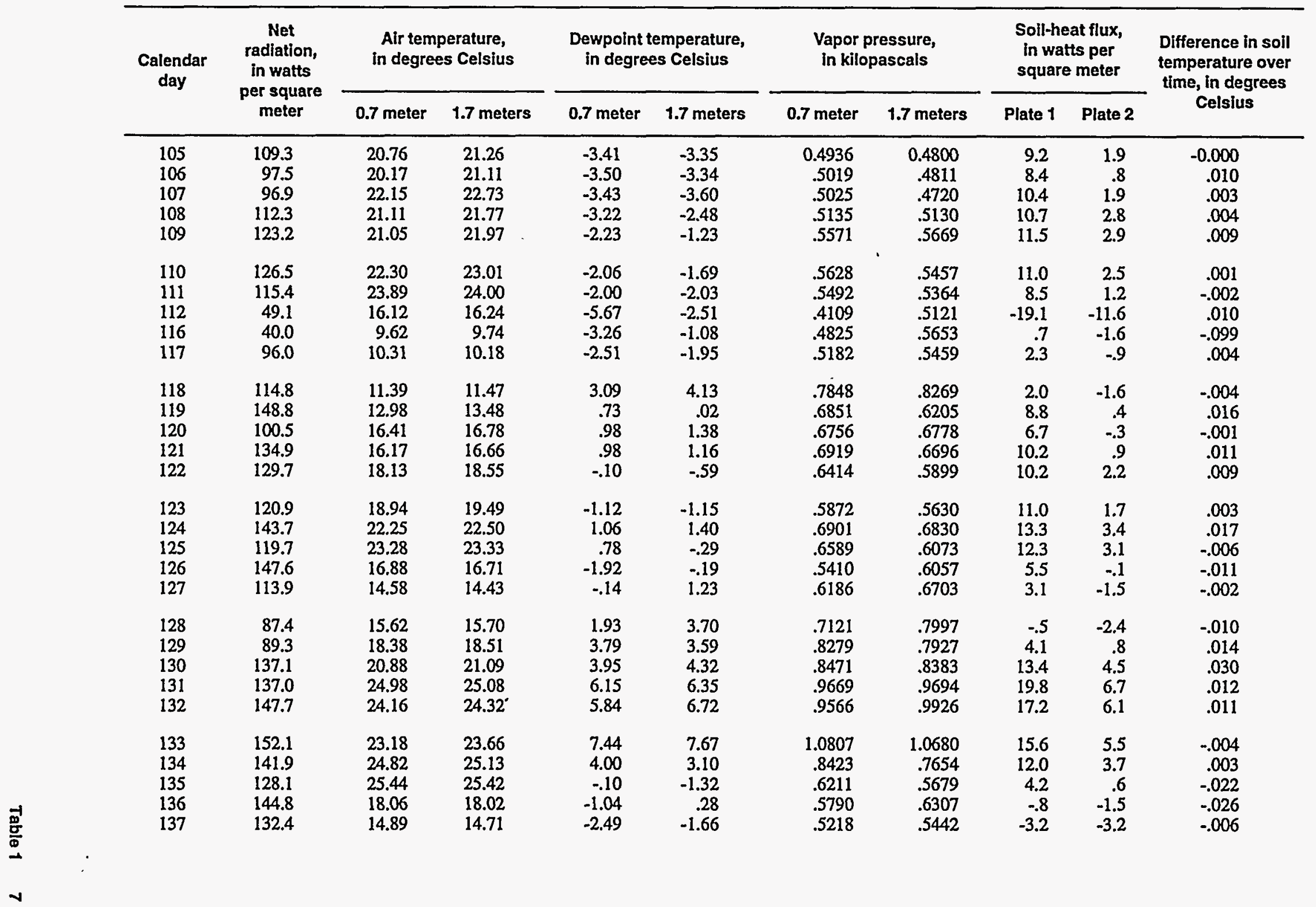


Table 1. Daily mean net radiation, air temperature, dew-point temperature, vapor pressure, soil heat flux, and soil temperature change, measured by energy-budget station at Rogers Spring Site 1, 1994—Continued

\begin{tabular}{|c|c|c|c|c|c|c|c|c|c|c|}
\hline \multirow{2}{*}{$\begin{array}{l}\text { Calendar } \\
\text { day }\end{array}$} & \multirow{2}{*}{$\begin{array}{c}\text { Net } \\
\text { radiation, } \\
\text { in watts } \\
\text { per square } \\
\text { meter }\end{array}$} & \multicolumn{2}{|c|}{$\begin{array}{l}\text { Air temperature, } \\
\text { in degrees Celsius }\end{array}$} & \multicolumn{2}{|c|}{$\begin{array}{l}\text { Dewpoint temperature, } \\
\text { in degrees Celsius }\end{array}$} & \multicolumn{2}{|c|}{$\begin{array}{l}\text { Vapor pressure, } \\
\text { in kilopascals }\end{array}$} & \multicolumn{2}{|c|}{$\begin{array}{l}\text { Soll-heat flux, } \\
\text { in watts per } \\
\text { square meter }\end{array}$} & \multirow{2}{*}{$\begin{array}{l}\text { Difference in soil } \\
\text { temperature over } \\
\text { time, in degrees } \\
\text { Celsius }\end{array}$} \\
\hline & & 0.7 meter & 1.7 meters & 0.7 meter & 1.7 meters & 0.7 meter & 1.7 meters & Plate 1 & Plate 2 & \\
\hline $\begin{array}{l}138 \\
139 \\
140 \\
141 \\
142\end{array}$ & $\begin{array}{l}149.8 \\
142.5 \\
148.2 \\
148.0 \\
148.7\end{array}$ & $\begin{array}{l}14.10 \\
13.78 \\
14.41 \\
17.16 \\
19.43\end{array}$ & $\begin{array}{l}13.89 \\
13.90 \\
14.94 \\
17.92 \\
20.22\end{array}$ & $\begin{array}{l}-1.90 \\
-1.05 \\
-2.91 \\
-5.00 \\
-5.22\end{array}$ & $\begin{array}{r}-0.70 \\
-.01 \\
-2.48 \\
-7.08 \\
-6.16\end{array}$ & $\begin{array}{r}0.5452 \\
.5916 \\
.5390 \\
.4537 \\
.4448\end{array}$ & $\begin{array}{r}0.5832 \\
.6159 \\
.5195 \\
.3621 \\
.3902\end{array}$ & $\begin{array}{l}0 \\
3.1 \\
1.4 \\
2.7 \\
6.1\end{array}$ & $\begin{array}{r}-1.2 \\
-.3 \\
-1.0 \\
-.3 \\
1.4\end{array}$ & $\begin{array}{r}-0.001 \\
-.002 \\
-.001 \\
.005 \\
.012\end{array}$ \\
\hline $\begin{array}{l}143 \\
144 \\
145 \\
146 \\
147\end{array}$ & $\begin{array}{r}153.9 \\
120.0 \\
130.1 \\
148.6 \\
95.3\end{array}$ & $\begin{array}{l}21.08 \\
23.28 \\
24.97 \\
24.53 \\
25.61\end{array}$ & $\begin{array}{l}21.96 \\
23.60 \\
24.94 \\
24.63 \\
25.71\end{array}$ & $\begin{array}{r}-2.19 \\
.62 \\
6.10 \\
8.57 \\
4.16\end{array}$ & $\begin{array}{r}-1.16 \\
.57 \\
6.83 \\
8.97 \\
2.06\end{array}$ & $\begin{array}{r}.5551 \\
.6660 \\
.9633 \\
1.1453 \\
.8322\end{array}$ & $\begin{array}{r}.5691 \\
.6518 \\
.9934 \\
1.1531 \\
.7119\end{array}$ & $\begin{array}{r}10.3 \\
8.3 \\
15.5 \\
15.2 \\
2.4\end{array}$ & $\begin{array}{l}3.2 \\
2.4 \\
6.1 \\
5.6 \\
0\end{array}$ & $\begin{array}{l}.014 \\
.017 \\
.009 \\
.006 \\
-.015\end{array}$ \\
\hline $\begin{array}{l}148 \\
149 \\
150 \\
151 \\
152\end{array}$ & $\begin{array}{l}148.2 \\
149.0 \\
100.0 \\
169.2 \\
162.0\end{array}$ & $\begin{array}{l}25.24 \\
24.87 \\
25.82 \\
21.55 \\
22.89\end{array}$ & $\begin{array}{l}25.56 \\
25.55 \\
26.07 \\
21.90 \\
23.46\end{array}$ & $\begin{array}{r}1.69 \\
-2.42 \\
.40 \\
7.31 \\
5.14\end{array}$ & $\begin{array}{r}-.78 \\
-5.10 \\
1.84 \\
8.79 \\
4.93\end{array}$ & $\begin{array}{r}.7220 \\
.5470 \\
.6524 \\
1.0544 \\
.9223\end{array}$ & $\begin{array}{r}.5973 \\
.4245 \\
.7449 \\
1.1542 \\
.8762\end{array}$ & $\begin{array}{r}11.7 \\
8.6 \\
6.6 \\
14.1 \\
10.9\end{array}$ & $\begin{array}{l}4.5 \\
3.3 \\
1.5 \\
5.4 \\
4.2\end{array}$ & $\begin{array}{r}.001 \\
.005 \\
.012 \\
-.004 \\
.005\end{array}$ \\
\hline $\begin{array}{l}153 \\
154 \\
155 \\
156 \\
157\end{array}$ & $\begin{array}{l}155.2 \\
119.2 \\
149.4 \\
155.2 \\
127.1\end{array}$ & $\begin{array}{l}25.73 \\
25.91 \\
23.86 \\
25.70 \\
23.58\end{array}$ & $\begin{array}{l}26.29 \\
26.50 \\
24.69 \\
25.91 \\
23.58\end{array}$ & $\begin{array}{r}3.17 \\
-.30 \\
-3.75 \\
-.64 \\
1.67\end{array}$ & $\begin{array}{r}1.90 \\
-2.45 \\
-3.62 \\
-.10 \\
2.02\end{array}$ & $\begin{array}{l}.8112 \\
.6362 \\
.4971 \\
.6032 \\
.7148\end{array}$ & $\begin{array}{l}.7097 \\
.5413 \\
.4760 \\
.6119 \\
.7342\end{array}$ & $\begin{array}{r}10.6 \\
2.7 \\
8.0 \\
8.3 \\
2.2\end{array}$ & $\begin{array}{r}4.0 \\
.8 \\
3.6 \\
4.2 \\
1.5\end{array}$ & $\begin{array}{r}.004 \\
-.010 \\
.005 \\
.009 \\
-.016\end{array}$ \\
\hline $\begin{array}{l}158 \\
159 \\
160 \\
161 \\
162\end{array}$ & $\begin{array}{l}155.4 \\
156.2 \\
160.2 \\
162.9 \\
159.8\end{array}$ & $\begin{array}{l}20.77 \\
23.57 \\
23.93 \\
26.29 \\
28.46\end{array}$ & $\begin{array}{l}21.66 \\
24.23 \\
24.83 \\
27.32 \\
29.17\end{array}$ & $\begin{array}{r}-1.66 \\
-1.37 \\
-1.44 \\
-1.18 \\
.88\end{array}$ & $\begin{array}{r}-1.25 \\
-2.16 \\
-1.48 \\
-2.03 \\
1.04\end{array}$ & $\begin{array}{l}.5668 \\
.5754 \\
.5874 \\
.5948 \\
.6884\end{array}$ & $\begin{array}{l}.5607 \\
.5390 \\
.5805 \\
.5340 \\
.6663\end{array}$ & $\begin{array}{r}4.8 \\
5.9 \\
9.0 \\
10.9 \\
12.1\end{array}$ & $\begin{array}{l}2.3 \\
3.4 \\
4.5 \\
5.6 \\
6.4\end{array}$ & $\begin{array}{l}.000 \\
.006 \\
.010 \\
.010 \\
.012\end{array}$ \\
\hline $\begin{array}{l}163 \\
164 \\
165 \\
166 \\
167\end{array}$ & $\begin{array}{l}156.0 \\
139.9 \\
154.6 \\
151.8 \\
154.5\end{array}$ & $\begin{array}{l}30.19 \\
29.90 \\
28.35 \\
25.26 \\
22.94\end{array}$ & $\begin{array}{l}30.41 \\
30.02 \\
28.20 \\
25.17 \\
23.30\end{array}$ & $\begin{array}{r}2.15 \\
1.83 \\
3.80 \\
.37 \\
-2.85\end{array}$ & $\begin{array}{r}2.38 \\
1.40 \\
4.86 \\
.04 \\
-2.81\end{array}$ & $\begin{array}{l}.7365 \\
.7144 \\
.8309 \\
.6460 \\
.5195\end{array}$ & $\begin{array}{l}.7331 \\
.6868 \\
.8822 \\
.6260 \\
.5065\end{array}$ & $\begin{array}{r}13.5 \\
10.3 \\
12.6 \\
5.4 \\
3.6\end{array}$ & $\begin{array}{l}7.3 \\
5.8 \\
7.8 \\
4.4 \\
3.0\end{array}$ & $\begin{array}{r}.008 \\
.004 \\
.001 \\
-.014 \\
-.011\end{array}$ \\
\hline
\end{tabular}


Table 1. Daily mean net radiation, air temperature, dew-point temperature, vapor pressure, soil heat flux, and soil temperature change, measured by energy-budget station at Rogers Spring Site 1, 1994-Continued

\begin{tabular}{|c|c|c|c|c|c|c|c|c|c|c|}
\hline \multirow{2}{*}{$\begin{array}{l}\text { Calendar } \\
\text { day }\end{array}$} & \multirow{2}{*}{$\begin{array}{c}\text { Net } \\
\text { radiation, } \\
\text { in watts } \\
\text { per square } \\
\text { meter }\end{array}$} & \multicolumn{2}{|c|}{$\begin{array}{l}\text { Air temperature, } \\
\text { in degrees Celsius }\end{array}$} & \multicolumn{2}{|c|}{$\begin{array}{l}\text { Dewpoint temperature, } \\
\text { in degrees Celsius }\end{array}$} & \multicolumn{2}{|c|}{$\begin{array}{l}\text { Vapor pressure, } \\
\text { in kilopascals }\end{array}$} & \multicolumn{2}{|c|}{$\begin{array}{l}\text { Soll-heat flux, } \\
\text { in watts per } \\
\text { square meter }\end{array}$} & \multirow{2}{*}{$\begin{array}{l}\text { Difference in soil } \\
\text { temperature over } \\
\text { time, in degrees } \\
\text { Celsius }\end{array}$} \\
\hline & & 0.7 meter & 1.7 meters & 0.7 meter & 1.7 meters & 0.7 meter & 1.7 meters & Plate 1 & Plate 2 & \\
\hline $\begin{array}{l}168 \\
169 \\
170 \\
171 \\
172\end{array}$ & $\begin{array}{l}157.3 \\
162.7 \\
164.7 \\
162.9 \\
159.7\end{array}$ & $\begin{array}{l}26.59 \\
26.48 \\
26.00 \\
27.28 \\
29.95\end{array}$ & $\begin{array}{l}26.93 \\
27.26 \\
26.85 \\
27.73 \\
30.28\end{array}$ & $\begin{array}{r}-2.61 \\
-2.42 \\
-2.13 \\
-.98 \\
4.77\end{array}$ & $\begin{array}{r}-3.48 \\
-1.99 \\
-2.19 \\
-.12 \\
4.95\end{array}$ & $\begin{array}{r}0.5226 \\
.5387 \\
.5596 \\
.6034 \\
.8776\end{array}$ & $\begin{array}{r}0.4800 \\
.5359 \\
.5306 \\
.6230 \\
.8862\end{array}$ & $\begin{array}{r}6.7 \\
8.2 \\
8.5 \\
10.6 \\
15.1\end{array}$ & $\begin{array}{l}4.9 \\
5.5 \\
5.1 \\
6.0 \\
8.0\end{array}$ & $\begin{array}{r}0.012 \\
-.005 \\
.002 \\
.024 \\
.001\end{array}$ \\
\hline $\begin{array}{l}173 \\
174 \\
175 \\
176 \\
177\end{array}$ & $\begin{array}{l}159.2 \\
156.1 \\
163.1 \\
155.2 \\
151.1\end{array}$ & $\begin{array}{l}30.51 \\
30.33 \\
28.68 \\
32.42 \\
34.75\end{array}$ & $\begin{array}{l}30.97 \\
30.88 \\
29.71 \\
32.79 \\
34.82\end{array}$ & $\begin{array}{r}2.56 \\
-.32 \\
.08 \\
1.50 \\
2.70\end{array}$ & $\begin{array}{r}1.75 \\
-1.49 \\
.83 \\
.94 \\
2.37\end{array}$ & $\begin{array}{l}.7626 \\
.6243 \\
.6588 \\
.7026 \\
.7548\end{array}$ & $\begin{array}{l}.7067 \\
.5574 \\
.6559 \\
.6594 \\
.7274\end{array}$ & $\begin{array}{l}11.8 \\
10.8 \\
10.4 \\
13.5 \\
18.2\end{array}$ & $\begin{array}{l}6.7 \\
6.0 \\
5.1 \\
7.1 \\
9.2\end{array}$ & $\begin{array}{l}. .001 \\
.000 \\
.007 \\
.017 \\
.009\end{array}$ \\
\hline $\begin{array}{l}178 \\
179 \\
180 \\
181 \\
182\end{array}$ & $\begin{array}{r}142.5 \\
166.7 \\
163.6 \\
148.6 \\
89.3\end{array}$ & $\begin{array}{l}32.86 \\
29.96 \\
32.75 \\
32.74 \\
32.65\end{array}$ & $\begin{array}{r}-64.25 \\
30.81 \\
33.30 \\
33.30 \\
32.96\end{array}$ & $\begin{array}{l}5.85 \\
5.46 \\
5.13 \\
4.18 \\
6.47\end{array}$ & $\begin{array}{l}7.34 \\
6.53 \\
4.61 \\
3.83 \\
7.43\end{array}$ & $\begin{array}{l}.9509 \\
.9300 \\
.9080 \\
.8457 \\
.9828\end{array}$ & $\begin{array}{r}1.0382 \\
.9840 \\
.8554 \\
.8051 \\
1.0381\end{array}$ & $\begin{array}{r}15.4 \\
14.4 \\
16.0 \\
14.9 \\
8.8\end{array}$ & $\begin{array}{l}7.6 \\
6.9 \\
7.7 \\
7.2 \\
4.0\end{array}$ & $\begin{array}{r}-.004 \\
-.003 \\
.015 \\
.008 \\
-.018\end{array}$ \\
\hline $\begin{array}{l}183 \\
184 \\
185 \\
186 \\
187\end{array}$ & $\begin{array}{l}153.2 \\
158.1 \\
162.4 \\
160.5 \\
163.7\end{array}$ & $\begin{array}{l}31.89 \\
27.71 \\
28.68 \\
28.20 \\
26.42\end{array}$ & $\begin{array}{l}31.92 \\
28.01 \\
28.83 \\
28.31 \\
27.31\end{array}$ & $\begin{array}{l}5.15 \\
2.62 \\
8.10 \\
7.92 \\
1.72\end{array}$ & $\begin{array}{l}4.03 \\
3.70 \\
9.33 \\
8.09 \\
1.66\end{array}$ & $\begin{array}{r}.9106 \\
.7812 \\
1.1142 \\
1.0939 \\
.7474\end{array}$ & $\begin{array}{r}.8398 \\
.8096 \\
1.1830 \\
1.0884 \\
.7409\end{array}$ & $\begin{array}{r}14.0 \\
9.6 \\
11.2 \\
9.8 \\
5.9\end{array}$ & $\begin{array}{l}7.1 \\
4.3 \\
4.9 \\
4.3 \\
1.9\end{array}$ & $\begin{array}{l}.001 \\
-.002 \\
-.001 \\
-.003 \\
-.021\end{array}$ \\
\hline $\begin{array}{l}188 \\
189 \\
190 \\
191 \\
192\end{array}$ & $\begin{array}{l}160.2 \\
164.2 \\
162.1 \\
160.4 \\
163.0\end{array}$ & $\begin{array}{l}28.55 \\
26.81 \\
29.99 \\
31.62 \\
28.63\end{array}$ & $\begin{array}{l}29.13 \\
28.07 \\
30.70 \\
31.91 \\
29.55\end{array}$ & $\begin{array}{r}-.51 \\
-.03 \\
.61 \\
1.81 \\
2.20\end{array}$ & $\begin{array}{l}-.25 \\
-.38 \\
.11 \\
1.99 \\
2.98\end{array}$ & $\begin{array}{l}.6085 \\
.6431 \\
.6702 \\
.7152 \\
.7477\end{array}$ & $\begin{array}{l}.6182 \\
.6104 \\
.6215 \\
.7100 \\
.7623\end{array}$ & $\begin{array}{r}8.9 \\
8.3 \\
9.8 \\
12.9 \\
10.0\end{array}$ & $\begin{array}{l}4.3 \\
3.4 \\
4.3 \\
6.3 \\
4.6\end{array}$ & $\begin{array}{r}.005 \\
.003 \\
.016 \\
.003 \\
. .008\end{array}$ \\
\hline $\begin{array}{l}193 \\
194 \\
195 \\
196 \\
197\end{array}$ & $\begin{array}{l}160.6 \\
161.0 \\
153.9 \\
163.4 \\
149.2\end{array}$ & $\begin{array}{l}29.02 \\
29.50 \\
30.20 \\
30.24 \\
30.14\end{array}$ & $\begin{array}{l}29.78 \\
30.14 \\
30.73 \\
30.92 \\
30.70\end{array}$ & $\begin{array}{l}3.28 \\
2.81 \\
3.76 \\
5.21 \\
8.43\end{array}$ & $\begin{array}{l}3.68 \\
2.86 \\
4.29 \\
5.84 \\
8.38\end{array}$ & $\begin{array}{r}.8062 \\
.7765 \\
.8355 \\
.9193 \\
1.1700\end{array}$ & $\begin{array}{r}.7973 \\
.7562 \\
.8389 \\
.9311 \\
1.1527\end{array}$ & $\begin{array}{r}10.3 \\
10.0 \\
9.8 \\
10.2 \\
9.3\end{array}$ & $\begin{array}{l}4.8 \\
4.8 \\
4.3 \\
4.7 \\
4.2\end{array}$ & $\begin{array}{r}.012 \\
-.013 \\
.008 \\
-.002 \\
.011\end{array}$ \\
\hline
\end{tabular}


Table 1. Daily mean net radiation, air temperature, dew-point temperature, vapor pressure, soil heat flux, and soil temperature change, measured by energy-budget station at Rogers Spring Site 1, 1994-Continued

\begin{tabular}{|c|c|c|c|c|c|c|c|c|c|c|}
\hline \multirow{2}{*}{$\begin{array}{l}\text { Calendar } \\
\text { day }\end{array}$} & \multirow{2}{*}{$\begin{array}{c}\text { Net } \\
\text { radiation, } \\
\text { in watts } \\
\text { per square } \\
\text { meter }\end{array}$} & \multicolumn{2}{|c|}{$\begin{array}{l}\text { Air temperature, } \\
\text { in degrees Celsius }\end{array}$} & \multicolumn{2}{|c|}{$\begin{array}{l}\text { Dewpoint temperature, } \\
\text { in degrees Celsius }\end{array}$} & \multicolumn{2}{|c|}{$\begin{array}{l}\text { Vapor pressure, } \\
\text { in kilopascals }\end{array}$} & \multicolumn{2}{|c|}{$\begin{array}{l}\text { Soil-heat flux, } \\
\text { in watts per } \\
\text { square meter }\end{array}$} & \multirow{2}{*}{$\begin{array}{c}\text { Difference in soil } \\
\text { temperature over } \\
\text { time, in degrees } \\
\text { Celsius }\end{array}$} \\
\hline & & 0.7 meter & 1.7 meters & 0.7 meter & 1.7 meters & 0.7 meter & 1.7 meters & Plate 1 & Plate 2 & \\
\hline $\begin{array}{l}198 \\
199 \\
200 \\
201 \\
202\end{array}$ & $\begin{array}{l}138.9 \\
150.0 \\
152.0 \\
143.2 \\
158.9\end{array}$ & $\begin{array}{l}30.97 \\
31.32 \\
28.66 \\
27.88 \\
29.10\end{array}$ & $\begin{array}{l}31.37 \\
31.25 \\
28.70 \\
17.91 \\
29.44\end{array}$ & $\begin{array}{l}19.01 \\
23.93 \\
25.11 \\
14.51 \\
11.33\end{array}$ & $\begin{array}{l}19.00 \\
23.86 \\
25.05 \\
14.23 \\
11.63\end{array}$ & $\begin{array}{l}2.2376 \\
2.9812 \\
3.2043 \\
1.7208 \\
1.3820\end{array}$ & $\begin{array}{l}2.2349 \\
2.9675 \\
3.1917 \\
1.6793 \\
1.3808\end{array}$ & $\begin{array}{r}14.4 \\
12.7 \\
11.3 \\
5.6 \\
8.8\end{array}$ & $\begin{array}{l}6.3 \\
6.1 \\
5.3 \\
2.0 \\
3.7\end{array}$ & $\begin{array}{r}0.006 \\
.014 \\
-.018 \\
-.009 \\
-.000\end{array}$ \\
\hline $\begin{array}{l}203 \\
204 \\
205 \\
206 \\
207\end{array}$ & $\begin{array}{l}167.8 \\
172.4 \\
163.2 \\
158.0 \\
153.5\end{array}$ & $\begin{array}{l}31.02 \\
30.27 \\
30.88 \\
29.98 \\
28.53\end{array}$ & $\begin{array}{l}31.24 \\
30.77 \\
31.26 \\
30.51 \\
29.49\end{array}$ & $\begin{array}{r}8.84 \\
8.02 \\
11.24 \\
24.45 \\
25.44\end{array}$ & $\begin{array}{r}8.16 \\
7.89 \\
10.51 \\
24.33 \\
25.38\end{array}$ & $\begin{array}{l}1.1565 \\
1.1011 \\
1.4437 \\
3.1463 \\
3.4194\end{array}$ & $\begin{array}{l}1.0868 \\
1.0667 \\
1.3718 \\
3.1218 \\
3.4030\end{array}$ & $\begin{array}{r}11.6 \\
12.2 \\
11.4 \\
9.6 \\
8.9\end{array}$ & $\begin{array}{l}5.1 \\
5.2 \\
4.8 \\
3.8 \\
3.5\end{array}$ & $\begin{array}{r}.010 \\
-.001 \\
-.010 \\
.011 \\
-.009\end{array}$ \\
\hline $\begin{array}{l}208 \\
209 \\
210 \\
211 \\
212\end{array}$ & $\begin{array}{l}163.6 \\
160.9 \\
140.5 \\
162.7 \\
160.5\end{array}$ & $\begin{array}{l}30.21 \\
31.14 \\
32.34 \\
32.68 \\
31.48\end{array}$ & $\begin{array}{l}30.81 \\
32.00 \\
32.67 \\
32.72 \\
31.70\end{array}$ & $\begin{array}{r}26.90 \\
28.57 \\
18.95 \\
9.01 \\
4.64\end{array}$ & $\begin{array}{r}26.81 \\
28.52 \\
19.43 \\
8.60 \\
4.11\end{array}$ & $\begin{array}{r}3.7638 \\
4.0603 \\
2.5437 \\
1.1681 \\
.8247\end{array}$ & $\begin{array}{r}3.7411 \\
4.0450 \\
2.5882 \\
1.1322 \\
.8247\end{array}$ & $\begin{array}{l}10.8 \\
12.0 \\
11.5 \\
14.6 \\
10.3\end{array}$ & $\begin{array}{l}4.3 \\
4.9 \\
5.0 \\
6.3 \\
4.2\end{array}$ & $\begin{array}{r}.014 \\
.003 \\
.014 \\
-.006 \\
-.008\end{array}$ \\
\hline $\begin{array}{l}213 \\
214 \\
215 \\
216 \\
217\end{array}$ & $\begin{array}{l}159.4 \\
155.8 \\
161.0 \\
160.8 \\
155.6\end{array}$ & $\begin{array}{l}32.05 \\
30.97 \\
27.27 \\
26.77 \\
30.60\end{array}$ & $\begin{array}{l}32.23 \\
31.22 \\
28.25 \\
27.54 \\
31.13\end{array}$ & $\begin{array}{r}2.91 \\
.10 \\
-2.15 \\
-3.39 \\
-.71\end{array}$ & $\begin{array}{r}2.31 \\
-1.05 \\
-1.15 \\
-3.54 \\
.29\end{array}$ & $\begin{array}{l}.7724 \\
.6355 \\
.5441 \\
.5190 \\
.6171\end{array}$ & $\begin{array}{l}.7260 \\
.5740 \\
.5803 \\
.4820 \\
.6366\end{array}$ & $\begin{array}{r}11.3 \\
9.0 \\
6.0 \\
4.6 \\
9.0\end{array}$ & $\begin{array}{l}4.7 \\
3.7 \\
2.0 \\
1.3 \\
2.9\end{array}$ & $\begin{array}{r}-.004 \\
-.004 \\
-.016 \\
-.009 \\
.032\end{array}$ \\
\hline $\begin{array}{l}218 \\
219 \\
220 \\
221 \\
222\end{array}$ & $\begin{array}{l}154.3 \\
149.6 \\
110.3 \\
147.2 \\
140.9\end{array}$ & $\begin{array}{l}33.27 \\
32.35 \\
31.77 \\
31.02 \\
30.30\end{array}$ & $\begin{array}{l}33.60 \\
32.85 \\
31.74 \\
30.82 \\
30.54\end{array}$ & $\begin{array}{r}3.61 \\
5.18 \\
7.64 \\
13.03 \\
14.63\end{array}$ & $\begin{array}{r}4.09 \\
5.53 \\
8.92 \\
14.12 \\
15.10\end{array}$ & $\begin{array}{r}.8184 \\
.9107 \\
1.0660 \\
1.5191 \\
1.6972\end{array}$ & $\begin{array}{r}.8271 \\
.9098 \\
1.1669 \\
1.6172 \\
1.7286\end{array}$ & $\begin{array}{r}13.7 \\
13.2 \\
8.5 \\
12.2 \\
9.3\end{array}$ & $\begin{array}{l}5.9 \\
5.4 \\
3.6 \\
5.2 \\
3.9\end{array}$ & $\begin{array}{r}.009 \\
.015 \\
-.007 \\
.010 \\
-.019\end{array}$ \\
\hline $\begin{array}{l}223 \\
224 \\
225 \\
226 \\
227\end{array}$ & $\begin{array}{l}160.4 \\
150.7 \\
149.3 \\
161.5 \\
159.7\end{array}$ & $\begin{array}{l}31.45 \\
32.64 \\
30.79 \\
29.45 \\
29.89\end{array}$ & $\begin{array}{l}31.78 \\
32.63 \\
30.94 \\
29.97 \\
30.81\end{array}$ & $\begin{array}{l}15.29 \\
26.68 \\
28.22 \\
26.38 \\
16.42\end{array}$ & $\begin{array}{l}15.08 \\
26.63 \\
28.22 \\
26.37 \\
15.89\end{array}$ & $\begin{array}{l}1.7691 \\
3.5335 \\
3.8434 \\
3.5169 \\
2.1122\end{array}$ & $\begin{array}{l}1.7521 \\
3.5236 \\
3.8412 \\
3.5121 \\
2.0411\end{array}$ & $\begin{array}{r}10.9 \\
9.3 \\
11.0 \\
7.6 \\
6.7\end{array}$ & $\begin{array}{l}4.3 \\
3.4 \\
4.1 \\
2.6 \\
2.4\end{array}$ & $\begin{array}{r}.004 \\
.019 \\
-.015 \\
-.004 \\
-.004\end{array}$ \\
\hline
\end{tabular}


Table 1. Daily mean net radiation, air temperature, dew-point temperature, vapor pressure, soil heat flux, and soil temperature change, measured by energy-budget station at Rogers Spring Site 1, 1994-Continued

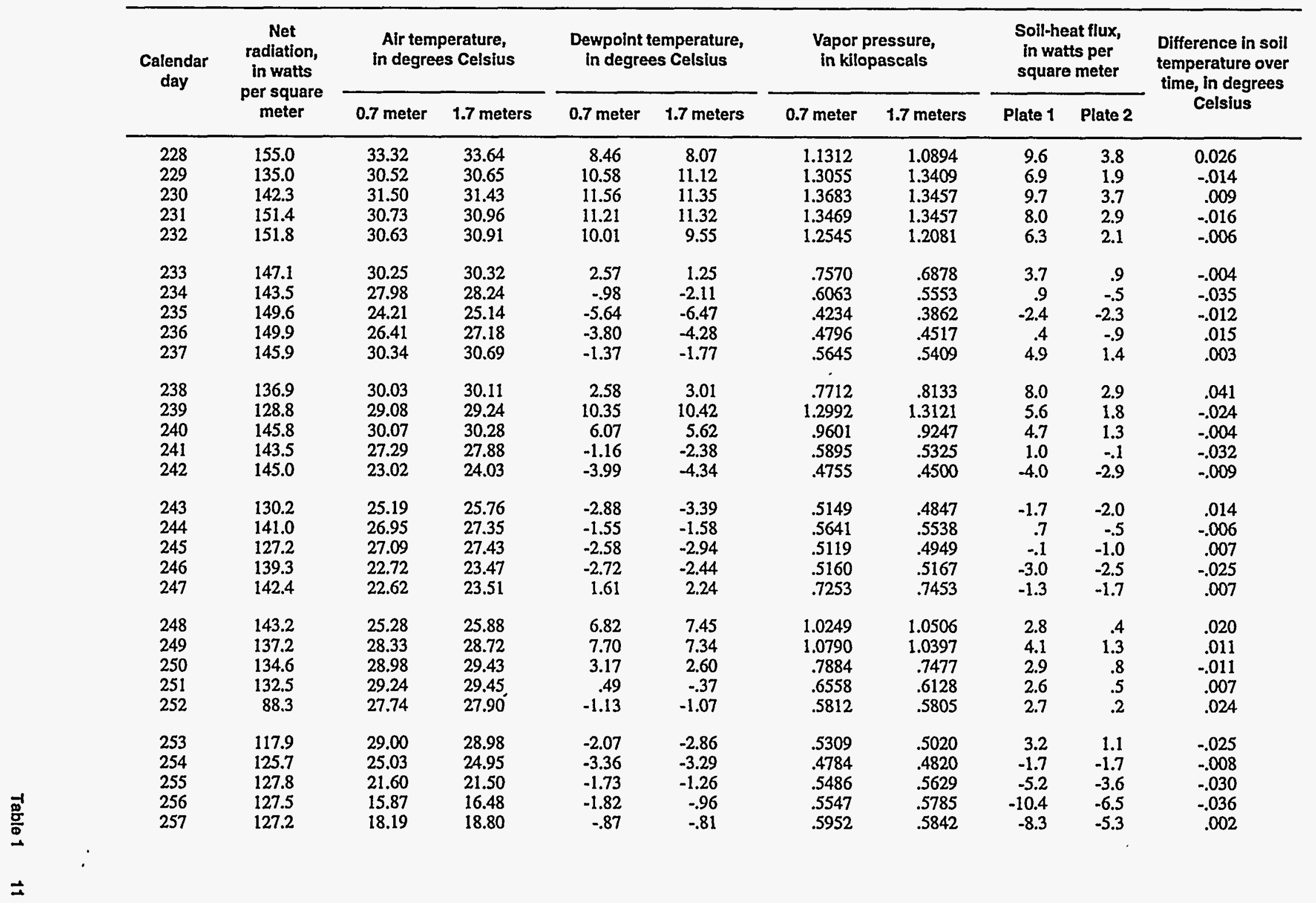


Table 1. Daily mean net radiation, air temperature, dew-point temperature, vapor pressure, soil heat flux, and soil temperature change, measured by energy-budget station at Rogers Spring Site 1, 1994-Continued

\begin{tabular}{|c|c|c|c|c|c|c|c|c|c|c|}
\hline \multirow[t]{2}{*}{$\begin{array}{l}\text { Calendar } \\
\text { day }\end{array}$} & \multirow{2}{*}{$\begin{array}{c}\text { Net } \\
\text { radiation, } \\
\text { in watts } \\
\text { per square } \\
\text { meter }\end{array}$} & \multicolumn{2}{|c|}{$\begin{array}{l}\text { Air temperature, } \\
\text { in degrees Celsius }\end{array}$} & \multicolumn{2}{|c|}{$\begin{array}{l}\text { Dewpoint temperature, } \\
\text { in degrees Celsius }\end{array}$} & \multicolumn{2}{|c|}{$\begin{array}{l}\text { Vapor pressure, } \\
\text { in kilopascals }\end{array}$} & \multicolumn{2}{|c|}{$\begin{array}{l}\text { Soll-heat flux, } \\
\text { in watts per } \\
\text { square meter }\end{array}$} & \multirow{2}{*}{$\begin{array}{c}\text { Difference in soil } \\
\text { temperature over } \\
\text { time, in degrees } \\
\text { Celsius }\end{array}$} \\
\hline & & 0.7 meter & 1.7 meters & 0.7 meter & 1.7 meters & 0.7 meter & 1.7 meters & Plate 1 & Plate 2 & \\
\hline $\begin{array}{l}258 \\
259 \\
260 \\
261 \\
262\end{array}$ & $\begin{array}{r}128.8 \\
106.7 \\
103.1 \\
97.9 \\
87.2\end{array}$ & $\begin{array}{l}20.38 \\
20.51 \\
20.79 \\
21.28 \\
21.24\end{array}$ & $\begin{array}{l}21.22 \\
21.37 \\
21.32 \\
21.95 \\
21.51\end{array}$ & $\begin{array}{r}-2.28 \\
-2.06 \\
-1.95 \\
.95 \\
4.81\end{array}$ & $\begin{array}{l}-2.67 \\
-1.94 \\
-2.03 \\
1.21 \\
5.57\end{array}$ & $\begin{array}{r}0.5377 \\
.5563 \\
.5504 \\
.6756 \\
.8907\end{array}$ & $\begin{array}{r}0.5101 \\
.5467 \\
.5407 \\
.6791 \\
.9314\end{array}$ & $\begin{array}{l}-5.9 \\
-3.9 \\
-3.5 \\
-1.9 \\
-7.1\end{array}$ & $\begin{array}{l}-3.9 \\
-3.2 \\
-2.7 \\
-2.0 \\
-4.7\end{array}$ & $\begin{array}{r}0.006 \\
.003 \\
.004 \\
.002 \\
-.001\end{array}$ \\
\hline $\begin{array}{l}263 \\
264 \\
265 \\
266 \\
267\end{array}$ & $\begin{array}{r}119.1 \\
123.8 \\
117.5 \\
120.4 \\
93.8\end{array}$ & $\begin{array}{l}23.34 \\
22.34 \\
21.35 \\
21.66 \\
23.82\end{array}$ & $\begin{array}{l}23.29 \\
23.06 \\
22.07 \\
22.24 \\
24.33\end{array}$ & $\begin{array}{r}6.92 \\
3.76 \\
1.90 \\
.42 \\
5.87\end{array}$ & $\begin{array}{r}7.03 \\
3.85 \\
1.73 \\
.19 \\
6.40\end{array}$ & $\begin{array}{r}1.0167 \\
.8192 \\
.7258 \\
.6522 \\
.9507\end{array}$ & $\begin{array}{r}1.0116 \\
.8137 \\
.7015 \\
.6296 \\
.9745\end{array}$ & $\begin{array}{r}-.4 \\
-3.1 \\
-3.6 \\
-2.6 \\
.8\end{array}$ & $\begin{array}{r}-1.2 \\
-2.2 \\
-2.6 \\
-2.1 \\
-.9\end{array}$ & $\begin{array}{r}.016 \\
-.016 \\
-.002 \\
.015 \\
-.004\end{array}$ \\
\hline $\begin{array}{l}268 \\
269 \\
270 \\
271 \\
272\end{array}$ & $\begin{array}{r}120.3 \\
111.8 \\
108.1 \\
68.7 \\
47.0\end{array}$ & $\begin{array}{l}20.53 \\
20.56 \\
22.77 \\
24.72 \\
22.09\end{array}$ & $\begin{array}{l}21.43 \\
21.17 \\
23.24 \\
25.11 \\
22.31\end{array}$ & $\begin{array}{r}2.59 \\
.15 \\
2.12 \\
7.28 \\
8.19\end{array}$ & $\begin{array}{r}2.52 \\
.21 \\
2.37 \\
7.81 \\
8.39\end{array}$ & $\begin{array}{l}.7678 \\
.6407 \\
.7433 \\
1.0422 \\
1.1306\end{array}$ & $\begin{array}{r}.7461 \\
.6399 \\
.7525 \\
1.0701 \\
1.1531\end{array}$ & $\begin{array}{r}-4.2 \\
-5.1 \\
-1.3 \\
1.8 \\
-5.4\end{array}$ & $\begin{array}{r}-2.9 \\
-3.4 \\
-1.6 \\
-.4 \\
-3.8\end{array}$ & $\begin{array}{r}-.015 \\
.004 \\
.021 \\
.014 \\
-.046\end{array}$ \\
\hline $\begin{array}{l}273 \\
274 \\
275 \\
276 \\
277\end{array}$ & $\begin{array}{r}87.7 \\
77.3 \\
66.1 \\
-.8 \\
43.8\end{array}$ & $\begin{array}{l}18.04 \\
17.68 \\
20.58 \\
18.52 \\
18.24\end{array}$ & $\begin{array}{l}18.62 \\
18.35 \\
21.01 \\
18.99 \\
18.16\end{array}$ & $\begin{array}{r}2.35 \\
2.12 \\
2.59 \\
.27 \\
4.12\end{array}$ & $\begin{array}{r}2.56 \\
2.42 \\
2.32 \\
.91 \\
4.77\end{array}$ & $\begin{array}{l}.7475 \\
.7461 \\
.7596 \\
.6305 \\
.8246\end{array}$ & $\begin{array}{l}.7464 \\
.7434 \\
.7302 \\
.6594 \\
.8606\end{array}$ & $\begin{array}{l}-9.4 \\
-7.8 \\
-4.7 \\
-9.3 \\
-4.6\end{array}$ & $\begin{array}{l}-5.7 \\
-4.8 \\
-3.1 \\
-6.1 \\
-3.4\end{array}$ & $\begin{array}{r}-.022 \\
.014 \\
.019 \\
-.020 \\
.004\end{array}$ \\
\hline $\begin{array}{l}278 \\
279 \\
280 \\
281 \\
282\end{array}$ & $\begin{array}{l}65.2 \\
72.0 \\
61.1 \\
56.1 \\
63.9\end{array}$ & $\begin{array}{l}13.97 \\
13.47 \\
17.36 \\
18.13 \\
15.13\end{array}$ & $\begin{array}{l}14.50 \\
13.98 \\
17.82 \\
18.79 \\
15.93\end{array}$ & $\begin{array}{r}8.18 \\
15.27 \\
4.62 \\
-1.48 \\
-4.83\end{array}$ & $\begin{array}{r}8.33 \\
15.27 \\
4.44 \\
-1.52 \\
-4.46\end{array}$ & $\begin{array}{r}1.2045 \\
2.0753 \\
1.0078 \\
.5668 \\
.4366\end{array}$ & $\begin{array}{r}1.2110 \\
2.0747 \\
1.0027 \\
.5532 \\
.4454\end{array}$ & $\begin{array}{r}-10.2 \\
-12.5 \\
-9.4 \\
-7.0 \\
-9.8\end{array}$ & $\begin{array}{l}-6.1 \\
-7.6 \\
-5.8 \\
-4.2 \\
-5.6\end{array}$ & $\begin{array}{r}-.051 \\
.011 \\
.009 \\
-.018 \\
.002\end{array}$ \\
\hline $\begin{array}{l}283 \\
284 \\
285 \\
286 \\
287\end{array}$ & $\begin{array}{l}63.0 \\
52.4 \\
53.9 \\
50.3 \\
34.4\end{array}$ & $\begin{array}{l}16.08 \\
18.14 \\
19.18 \\
14.83 \\
16.36\end{array}$ & $\begin{array}{l}17.23 \\
18.93 \\
19.50 \\
15.29 \\
16.44\end{array}$ & $\begin{array}{r}-5.37 \\
-5.32 \\
-6.87 \\
-3.01 \\
1.86\end{array}$ & $\begin{array}{r}-5.11 \\
-4.84 \\
-6.66 \\
-2.91 \\
2.54\end{array}$ & $\begin{array}{l}.4172 \\
.4166 \\
.3687 \\
.5133 \\
.7075\end{array}$ & $\begin{array}{l}.4219 \\
.4290 \\
.3743 \\
.5122 \\
.7376\end{array}$ & $\begin{array}{r}-8.6 \\
-6.1 \\
-3.9 \\
-10.9 \\
-5.4\end{array}$ & $\begin{array}{l}-5.1 \\
-3.7 \\
-2.6 \\
-6.5 \\
-3.9\end{array}$ & $\begin{array}{r}.001 \\
-.007 \\
.035 \\
-.022 \\
-.000\end{array}$ \\
\hline
\end{tabular}


Table 1. Daily mean net radiation, air temperature, dew-point temperature, vapor pressure, soil heat flux, and soil temperature change, measured by energy-budget station at Rogers Spring Site 1, 1994-Continued

\begin{tabular}{|c|c|c|c|c|c|c|c|c|c|c|}
\hline \multirow{2}{*}{$\begin{array}{l}\text { Calendar } \\
\text { day }\end{array}$} & \multirow{2}{*}{$\begin{array}{c}\text { Net } \\
\text { radiation, } \\
\text { in watts } \\
\text { per square } \\
\text { meter }\end{array}$} & \multicolumn{2}{|c|}{$\begin{array}{l}\text { Air temperature, } \\
\text { in degrees Celsius }\end{array}$} & \multicolumn{2}{|c|}{$\begin{array}{l}\text { Dewpoint temperature, } \\
\text { in degrees Celsius }\end{array}$} & \multicolumn{2}{|c|}{$\begin{array}{l}\text { Vapor pressure, } \\
\text { in kilopascals }\end{array}$} & \multicolumn{2}{|c|}{$\begin{array}{l}\text { Soil-heat flux, } \\
\text { in watts per } \\
\text { square meter }\end{array}$} & \multirow{2}{*}{$\begin{array}{c}\text { Difference in soil } \\
\text { temperature over } \\
\text { time, in degrees } \\
\text { Celsius }\end{array}$} \\
\hline & & 0.7 meter & 1.7 meters & 0.7 meter & 1.7 meters & 0.7 meter & 1.7 meters & Plate 1 & Plate 2 & \\
\hline $\begin{array}{l}288 \\
289 \\
290 \\
291 \\
292\end{array}$ & $\begin{array}{l}50.1 \\
49.1 \\
54.7 \\
55.6 \\
52.6\end{array}$ & $\begin{array}{r}12.48 \\
10.65 \\
9.39 \\
10.27 \\
10.66\end{array}$ & $\begin{array}{r}12.46 \\
10.74 \\
9.92 \\
11.00 \\
11.58\end{array}$ & $\begin{array}{l}-1.99 \\
-4.55 \\
-3.70 \\
-4.69 \\
-5.37\end{array}$ & $\begin{array}{l}-1.96 \\
-4.53 \\
-3.70 \\
-4.69 \\
-5.37\end{array}$ & $\begin{array}{r}0.5390 \\
.4471 \\
.4846 \\
.4493 \\
.4354\end{array}$ & $\begin{array}{r}0.5381 \\
.4409 \\
.4778 \\
.4404 \\
.4200\end{array}$ & $\begin{array}{l}-13.2 \\
-15.4 \\
-14.5 \\
-12.9 \\
-12.6\end{array}$ & $\begin{array}{l}-8.1 \\
-8.7 \\
-8.1 \\
-7.3 \\
-7.1\end{array}$ & $\begin{array}{r}-0.042 \\
.007 \\
-.022 \\
-.001 \\
-.002\end{array}$ \\
\hline $\begin{array}{l}293 \\
294 \\
295 \\
296 \\
297\end{array}$ & $\begin{array}{l}50.0 \\
50.5 \\
35.1 \\
46.5 \\
50.8\end{array}$ & $\begin{array}{l}10.93 \\
10.86 \\
11.13 \\
13.16 \\
14.51\end{array}$ & $\begin{array}{l}11.62 \\
11.82 \\
12.00 \\
13.87 \\
15.21\end{array}$ & $\begin{array}{l}-5.83 \\
-6.39 \\
-6.36 \\
-5.54 \\
-3.18\end{array}$ & $\begin{array}{l}-5.64 \\
-6.34 \\
-6.25 \\
-5.63 \\
-2.76\end{array}$ & $\begin{array}{l}.4172 \\
.4072 \\
.4025 \\
.4249 \\
.4995\end{array}$ & $\begin{array}{l}.4131 \\
.3937 \\
.3966 \\
.4123 \\
.5068\end{array}$ & $\begin{array}{r}-12.1 \\
-12.0 \\
-10.8 \\
-8.2 \\
-5.8\end{array}$ & $\begin{array}{l}-6.8 \\
-6.7 \\
-6.4 \\
-4.9 \\
-3.5\end{array}$ & $\begin{array}{r}.001 \\
-.002 \\
.001 \\
.030 \\
-.011\end{array}$ \\
\hline $\begin{array}{l}298 \\
299 \\
300 \\
301 \\
302\end{array}$ & $\begin{array}{l}47.9 \\
43.8 \\
40.3 \\
41.9 \\
37.3\end{array}$ & $\begin{array}{l}13.44 \\
13.19 \\
13.37 \\
12.08 \\
13.12\end{array}$ & $\begin{array}{l}14.41 \\
14.04 \\
14.38 \\
13.19 \\
13.82\end{array}$ & $\begin{array}{l}-3.45 \\
-3.35 \\
-3.57 \\
-4.27 \\
-4.58\end{array}$ & $\begin{array}{l}-2.74 \\
-3.04 \\
-3.13 \\
-4.00 \\
-4.55\end{array}$ & $\begin{array}{l}.5120 \\
.5094 \\
.5002 \\
.4769 \\
.4659\end{array}$ & $\begin{array}{l}.5120 \\
.5059 \\
.5024 \\
.4705 \\
.4568\end{array}$ & $\begin{array}{r}-7.9 \\
-8.2 \\
-7.9 \\
-9.9 \\
-10.0\end{array}$ & $\begin{array}{l}-4.9 \\
-5.1 \\
-5.1 \\
-6.0 \\
-6.2\end{array}$ & $\begin{array}{r}-.004 \\
-.003 \\
-.002 \\
-.011 \\
.012\end{array}$ \\
\hline $\begin{array}{l}303 \\
304 \\
305 \\
306 \\
307\end{array}$ & $\begin{array}{r}27.3 \\
35.9 \\
22.2 \\
7.8 \\
20.7\end{array}$ & $\begin{array}{r}12.72 \\
9.48 \\
14.30 \\
13.42 \\
6.51\end{array}$ & $\begin{array}{r}13.67 \\
10.51 \\
14.49 \\
13.59 \\
6.49\end{array}$ & $\begin{array}{r}-7.43 \\
-12.03 \\
-9.91 \\
-3.48 \\
-7.22\end{array}$ & $\begin{array}{r}-8.15 \\
-12.74 \\
-9.90 \\
-2.93 \\
-7.26\end{array}$ & $\begin{array}{l}.3685 \\
.2884 \\
.3151 \\
.4754 \\
.3657\end{array}$ & $\begin{array}{l}.3431 \\
.2661 \\
.3120 \\
.4947 \\
.3638\end{array}$ & $\begin{array}{r}-11.3 \\
-13.7 \\
-4.8 \\
-6.3 \\
-17.9\end{array}$ & $\begin{array}{r}-6.6 \\
-7.9 \\
-3.8 \\
-4.5 \\
-10.4\end{array}$ & $\begin{array}{r}-.036 \\
-.002 \\
.069 \\
-.037 \\
-.058\end{array}$ \\
\hline $\begin{array}{l}308 \\
309 \\
310 \\
311 \\
312\end{array}$ & $\begin{array}{r}28.6 \\
40.1 \\
30.4 \\
9.0 \\
39.6\end{array}$ & $\begin{array}{r}3.25 \\
6.95 \\
10.98 \\
9.06 \\
9.13\end{array}$ & $\begin{array}{r}3.88 \\
7.40 \\
11.56 \\
9.83 \\
9.83^{\circ}\end{array}$ & $\begin{array}{r}-12.22 \\
-11.65 \\
-8.70 \\
-9.94 \\
-5.19\end{array}$ & $\begin{array}{r}-12.37 \\
-11.79 \\
-8.46 \\
-9.71 \\
-4.44\end{array}$ & $\begin{array}{l}.2503 \\
.2604 \\
.3293 \\
.2962 \\
.4417\end{array}$ & $\begin{array}{l}.2422 \\
.2532 \\
.3291 \\
.2996 \\
.4637\end{array}$ & $\begin{array}{r}-19.5 \\
-13.0 \\
-9.2 \\
-11.4 \\
-9.2\end{array}$ & $\begin{array}{r}-11.5 \\
-8.0 \\
-6.0 \\
-7.3 \\
-6.1\end{array}$ & $\begin{array}{r}-.010 \\
.014 \\
.024 \\
-.027 \\
.021\end{array}$ \\
\hline $\begin{array}{l}313 \\
314 \\
315 \\
316 \\
317\end{array}$ & $\begin{array}{r}29.0 \\
3.8 \\
31.7 \\
46.6 \\
10.8\end{array}$ & $\begin{array}{r}11.57 \\
9.11 \\
3.93 \\
3.31 \\
6.25\end{array}$ & $\begin{array}{r}11.84 \\
9.28 \\
4.34 \\
3.96 \\
6.27\end{array}$ & $\begin{array}{l}-4.62 \\
-3.30 \\
-2.99 \\
-3.80 \\
-5.53\end{array}$ & $\begin{array}{l}-4.00 \\
-2.70 \\
-2.32 \\
-3.51 \\
-6.26\end{array}$ & $\begin{array}{l}.4436 \\
.4906 \\
.5138 \\
.4945 \\
.4134\end{array}$ & $\begin{array}{l}.4639 \\
.5191 \\
.5343 \\
.5001 \\
.3883\end{array}$ & $\begin{array}{r}-7.1 \\
-9.9 \\
-16.6 \\
-16.7 \\
-15.7\end{array}$ & $\begin{array}{r}-5.1 \\
-6.6 \\
-10.6 \\
-10.3 \\
-9.6\end{array}$ & $\begin{array}{r}.024 \\
-.040 \\
-.009 \\
.002 \\
-.018\end{array}$ \\
\hline $\begin{array}{l}318 \\
319 \\
320\end{array}$ & $\begin{array}{l}15.1 \\
27.0 \\
25.6\end{array}$ & $\begin{array}{l}3.75 \\
3.45 \\
8.08\end{array}$ & $\begin{array}{l}4.25 \\
4.05 \\
8.13\end{array}$ & $\begin{array}{r}-9.23 \\
1.68 \\
10.53\end{array}$ & $\begin{array}{r}-9.41 \\
2.25 \\
10.54\end{array}$ & $\begin{array}{r}.3161 \\
1.0048 \\
1.3289\end{array}$ & $\begin{array}{r}.3058 \\
1.0124 \\
1.3299\end{array}$ & $\begin{array}{r}-18.4 \\
-14.9 \\
-7.4\end{array}$ & $\begin{array}{r}-10.8 \\
-9.5 \\
-5.4\end{array}$ & $\begin{array}{r}-.033 \\
.051 \\
-.025\end{array}$ \\
\hline
\end{tabular}


Table 2. Daily mean, maximum, and minimum air and dew-point temperatures and vapor pressure measured by energybudget station at Rogers Spring Site 1, 1994

\begin{tabular}{|c|c|c|c|c|c|c|c|c|c|}
\hline \multirow{2}{*}{$\begin{array}{l}\text { Calendar } \\
\text { day }\end{array}$} & \multicolumn{3}{|c|}{$\begin{array}{c}\text { Alr temperature at } \\
0.7 \text { meters, in degrees Celsius }\end{array}$} & \multicolumn{3}{|c|}{$\begin{array}{l}\text { Dew-polnt temperature at } \\
0.7 \text { meter, in degrees Celsius }\end{array}$} & \multicolumn{3}{|c|}{$\begin{array}{l}\text { Vapor pressure at } 0.7 \text { meter, } \\
\text { in kilopascals }\end{array}$} \\
\hline & Mean & Maximum & Minimum & Mean & Maximum & Minimum & Mean & Maximum & Minimum \\
\hline $\begin{array}{l}75 \\
76 \\
77 \\
78 \\
79 \\
80\end{array}$ & $\begin{array}{l}21.41 \\
18.05 \\
18.58 \\
14.63 \\
11.87 \\
14.10\end{array}$ & $\begin{array}{l}26.74 \\
24.68 \\
23.51 \\
18.97 \\
20.31 \\
22.17\end{array}$ & $\begin{array}{r}17.10 \\
9.83 \\
13.99 \\
8.62 \\
5.39 \\
2.01\end{array}$ & $\begin{array}{r}-6.75 \\
-6.97 \\
-3.16 \\
.75 \\
.24 \\
-.08\end{array}$ & $\begin{array}{r}1.10 \\
-2.52 \\
.92 \\
4.67 \\
6.86 \\
6.04\end{array}$ & $\begin{array}{r}-10.37 \\
-10.83 \\
-9.07 \\
-2.97 \\
-6.91 \\
-8.22\end{array}$ & $\begin{array}{r}0.4043 \\
.3715 \\
.4939 \\
.6556 \\
.6547 \\
.6440\end{array}$ & $\begin{array}{r}1.1655 \\
.5082 \\
.6528 \\
.8520 \\
.9917 \\
.9369\end{array}$ & $\begin{array}{r}0.2785 \\
.2684 \\
.3082 \\
.4910 \\
.3646 \\
.3302\end{array}$ \\
\hline $\begin{array}{l}81 \\
82 \\
83 \\
84 \\
85\end{array}$ & $\begin{array}{r}12.45 \\
9.23 \\
11.86 \\
8.82 \\
11.28\end{array}$ & $\begin{array}{l}17.00 \\
16.48 \\
17.70 \\
14.92 \\
20.68\end{array}$ & $\begin{array}{r}.93 \\
-1.40 \\
7.42 \\
4.34 \\
-.54\end{array}$ & $\begin{array}{l}-4.05 \\
-9.64 \\
-5.47 \\
-3.64 \\
-2.71\end{array}$ & $\begin{array}{r}2.09 \\
-4.30 \\
-.26 \\
1.42 \\
3.89\end{array}$ & $\begin{array}{r}-14.98 \\
-16.26 \\
-9.23 \\
-7.09 \\
-8.72\end{array}$ & $\begin{array}{l}.4839 \\
.3081 \\
.4168 \\
.4779 \\
.5273\end{array}$ & $\begin{array}{l}.7102 \\
.4461 \\
.5996 \\
.6769 \\
.8064\end{array}$ & $\begin{array}{l}.1919 \\
.1726 \\
.3061 \\
.3594 \\
.3168\end{array}$ \\
\hline $\begin{array}{l}86 \\
87 \\
88 \\
89 \\
90\end{array}$ & $\begin{array}{l}14.00 \\
14.63 \\
15.03 \\
17.22 \\
16.52\end{array}$ & $\begin{array}{l}24.45 \\
24.91 \\
26.03 \\
27.11 \\
27.76\end{array}$ & $\begin{array}{r}3.56 \\
.29 \\
2.62 \\
7.08 \\
2.74\end{array}$ & $\begin{array}{l}-6.05 \\
-6.67 \\
-6.10 \\
-4.84 \\
-5.15\end{array}$ & $\begin{array}{r}-.93 \\
-.23 \\
.47 \\
.69 \\
.69\end{array}$ & $\begin{array}{r}-12.94 \\
-13.63 \\
-12.71 \\
-9.17 \\
-11.29\end{array}$ & $\begin{array}{l}.4058 \\
.3947 \\
.4109 \\
.4442 \\
.4462\end{array}$ & $\begin{array}{l}.5711 \\
.6006 \\
.6323 \\
.6424 \\
.7144\end{array}$ & $\begin{array}{l}.2267 \\
.2146 \\
.2310 \\
.3060 \\
.2588\end{array}$ \\
\hline $\begin{array}{l}91 \\
92 \\
93 \\
94 \\
95\end{array}$ & $\begin{array}{l}15.19 \\
17.03 \\
18.86 \\
14.84 \\
12.99\end{array}$ & $\begin{array}{l}25.71 \\
25.88 \\
24.48 \\
23.05 \\
21.56\end{array}$ & $\begin{array}{r}.60 \\
4.97 \\
11.35 \\
4.40 \\
3.24\end{array}$ & $\begin{array}{l}-6.52 \\
-6.08 \\
-2.94 \\
-5.75 \\
-9.38\end{array}$ & $\begin{array}{r}.03 \\
-.07 \\
1.34 \\
.68 \\
-3.79\end{array}$ & $\begin{array}{r}-12.81 \\
-11.64 \\
-7.37 \\
-12.34 \\
-15.58\end{array}$ & $\begin{array}{l}.3971 \\
.4078 \\
.5010 \\
.4181 \\
.3155\end{array}$ & $\begin{array}{l}.6121 \\
.6076 \\
.6730 \\
.6414 \\
.4620\end{array}$ & $\begin{array}{l}.2288 \\
.2515 \\
.3517 \\
.2377 \\
.1824\end{array}$ \\
\hline $\begin{array}{r}96 \\
97 \\
98 \\
99 \\
100\end{array}$ & $\begin{array}{l}14.70 \\
14.90 \\
14.85 \\
11.50 \\
13.84\end{array}$ & $\begin{array}{l}22.84 \\
22.71 \\
19.97 \\
17.85 \\
21.39\end{array}$ & $\begin{array}{l}3.85 \\
2.31 \\
9.58 \\
5.46 \\
5.83\end{array}$ & $\begin{array}{l}-7.10 \\
-5.43 \\
-4.19 \\
-4.00 \\
-3.81\end{array}$ & $\begin{array}{r}-2.21 \\
.45 \\
.42 \\
1.62 \\
1.04\end{array}$ & $\begin{array}{r}-14.05 \\
-10.27 \\
-7.71 \\
-8.35 \\
-8.32\end{array}$ & $\begin{array}{l}.3751 \\
.4205 \\
.4572 \\
.4687 \\
.4736\end{array}$ & $\begin{array}{l}.5197 \\
.6312 \\
.6299 \\
.6865 \\
.6585\end{array}$ & $\begin{array}{l}.2071 \\
.2806 \\
.3446 \\
.3262 \\
.3269\end{array}$ \\
\hline $\begin{array}{l}101 \\
102 \\
103 \\
104 \\
105\end{array}$ & $\begin{array}{l}14.26 \\
15.54 \\
17.36 \\
18.62 \\
20.76\end{array}$ & $\begin{array}{l}24.23 \\
26.44 \\
28.59 \\
29.56 \\
29.81\end{array}$ & $\begin{array}{l}2.79 \\
3.79 \\
2.92 \\
4.96 \\
9.95\end{array}$ & $\begin{array}{l}-3.76 \\
-3.86 \\
-5.07 \\
-3.61 \\
-3.41\end{array}$ & $\begin{array}{l}2.97 \\
4.02 \\
2.21 \\
3.42 \\
1.98\end{array}$ & $\begin{array}{r}-10.08 \\
-10.06 \\
-12.44 \\
-11.50 \\
-9.25\end{array}$ & $\begin{array}{l}.4918 \\
.4968 \\
.4513 \\
.4996 \\
.4936\end{array}$ & $\begin{array}{l}.7561 \\
.8142 \\
.7165 \\
.7803 \\
.7043\end{array}$ & $\begin{array}{l}.2849 \\
.2854 \\
.2363 \\
.2548 \\
.3041\end{array}$ \\
\hline $\begin{array}{l}106 \\
107 \\
108 \\
109 \\
110\end{array}$ & $\begin{array}{l}20.17 \\
22.15 \\
21.11 \\
21.05 \\
22.30\end{array}$ & $\begin{array}{l}31.95 \\
32.98 \\
33.20 \\
33.78 \\
34.57\end{array}$ & $\begin{array}{r}7.26 \\
11.99 \\
10.01 \\
8.22 \\
9.28\end{array}$ & $\begin{array}{l}-3.50 \\
-3.43 \\
-3.22 \\
-2.23 \\
-2.06\end{array}$ & $\begin{array}{l}3.64 \\
3.62 \\
3.96 \\
5.36 \\
5.75\end{array}$ & $\begin{array}{l}-9.71 \\
-8.80 \\
-9.10 \\
-9.21 \\
-8.37\end{array}$ & $\begin{array}{l}.5019 \\
.5025 \\
.5135 \\
.5571 \\
.5628\end{array}$ & $\begin{array}{l}.7928 \\
.7914 \\
.8107 \\
.8939 \\
.9185\end{array}$ & $\begin{array}{l}.2935 \\
.3146 \\
.3085 \\
.3050 \\
.3254\end{array}$ \\
\hline $\begin{array}{l}111 \\
112 \\
116 \\
117 \\
118\end{array}$ & $\begin{array}{r}23.89 \\
16.12 \\
9.62 \\
10.31 \\
11.39\end{array}$ & $\begin{array}{l}31.05 \\
21.88 \\
17.59 \\
16.27 \\
17.43\end{array}$ & $\begin{array}{r}16.35 \\
11.98 \\
5.36 \\
5.56 \\
5.75\end{array}$ & $\begin{array}{r}-2.00 \\
-5.67 \\
-3.26 \\
-2.51 \\
3.09\end{array}$ & $\begin{array}{r}3.88 \\
.34 \\
-.56 \\
2.19 \\
9.27\end{array}$ & $\begin{array}{l}-6.25 \\
-8.50 \\
-5.04 \\
-6.14 \\
-1.89\end{array}$ & $\begin{array}{l}.5492 \\
.4109 \\
.4825 \\
.5182 \\
.7848\end{array}$ & $\begin{array}{r}.8062 \\
.6261 \\
.5865 \\
.7152 \\
1.1684\end{array}$ & $\begin{array}{l}.3840 \\
.3222 \\
.4207 \\
.4009 \\
.5319\end{array}$ \\
\hline $\begin{array}{l}119 \\
120 \\
121 \\
122 \\
123\end{array}$ & $\begin{array}{l}12.98 \\
16.41 \\
16.17 \\
18.13 \\
18.94\end{array}$ & $\begin{array}{l}22.95 \\
23.43 \\
26.13 \\
28.22 \\
28.41\end{array}$ & $\begin{array}{l}1.49 \\
8.65 \\
6.96 \\
5.90 \\
7.74\end{array}$ & $\begin{array}{r}.73 \\
.98 \\
.98 \\
-.10 \\
-1.12\end{array}$ & $\begin{array}{l}7.52 \\
6.10 \\
7.74 \\
7.00 \\
4.39\end{array}$ & $\begin{array}{l}-5.70 \\
-3.49 \\
-4.90 \\
-6.00 \\
-7.55\end{array}$ & $\begin{array}{l}.6851 \\
.6756 \\
.6919 \\
.6414 \\
.5872\end{array}$ & $\begin{array}{r}1.0376 \\
.9412 \\
1.0531 \\
1.0012 \\
.8357\end{array}$ & $\begin{array}{l}.3996 \\
.4721 \\
.4250 \\
.3908 \\
.3469\end{array}$ \\
\hline $\begin{array}{l}124 \\
125 \\
126 \\
127 \\
128\end{array}$ & $\begin{array}{l}22.25 \\
23.28 \\
16.88 \\
14.58 \\
15.62\end{array}$ & $\begin{array}{l}30.96 \\
29.78 \\
22.69 \\
20.18 \\
21.04\end{array}$ & $\begin{array}{r}9.69 \\
17.49 \\
11.64 \\
7.86 \\
9.55\end{array}$ & $\begin{array}{r}1.06 \\
.78 \\
-1.92 \\
-.14 \\
1.93\end{array}$ & $\begin{array}{l}6.66 \\
4.49 \\
2.02 \\
4.45 \\
6.13\end{array}$ & $\begin{array}{l}-6.49 \\
-4.88 \\
-6.07 \\
-4.74 \\
-1.99\end{array}$ & $\begin{array}{l}.6901 \\
.6589 \\
.5410 \\
.6186 \\
.7121\end{array}$ & $\begin{array}{l}.9780 \\
.8414 \\
.7064 \\
.8389 \\
.9428\end{array}$ & $\begin{array}{r}.3764 \\
.4255 \\
.3886 \\
. .4300 \\
.5279\end{array}$ \\
\hline
\end{tabular}


Table 2. Daily mean, maximum, and minimum air and dew-point temperature and vapor pressure measured by energybudget station at Rogers Spring Slte 1,1994-Continued

\begin{tabular}{|c|c|c|c|c|c|c|c|c|c|}
\hline \multirow{2}{*}{$\begin{array}{l}\text { Calendar } \\
\text { day }\end{array}$} & \multicolumn{3}{|c|}{$\begin{array}{c}\text { Alr temperature at } \\
0.7 \text { meter, in degrees Celsius }\end{array}$} & \multicolumn{3}{|c|}{$\begin{array}{l}\text { Dew-point temperature at } \\
0.7 \text { meter, in degrees Celsius }\end{array}$} & \multicolumn{3}{|c|}{$\begin{array}{l}\text { Vapor pressure at } 0.7 \text { meter, } \\
\text { in kilopascals }\end{array}$} \\
\hline & Mean & Maximum & Minimum & Mean & Maximum & Minimum & Mean & Maximum & Minimum \\
\hline $\begin{array}{l}129 \\
130 \\
131 \\
132 \\
133\end{array}$ & $\begin{array}{l}18.38 \\
20.88 \\
24.98 \\
24.16 \\
23.18\end{array}$ & $\begin{array}{l}28.13 \\
30.57 \\
33.63 \\
32.87 \\
33.10\end{array}$ & $\begin{array}{r}7.32 \\
7.48 \\
14.10 \\
11.13 \\
11.12\end{array}$ & $\begin{array}{l}3.79 \\
3.95 \\
6.15 \\
5.84 \\
7.44\end{array}$ & $\begin{array}{r}9.27 \\
9.68 \\
11.26 \\
10.46 \\
13.91\end{array}$ & $\begin{array}{r}-1.88 \\
-3.95 \\
1.66 \\
-1.47 \\
.32\end{array}$ & $\begin{array}{r}0.8279 \\
.8471 \\
.9669 \\
.9566 \\
1.0807\end{array}$ & $\begin{array}{l}1.1684 \\
1.2010 \\
1.3346 \\
1.2651 \\
1.5886\end{array}$ & $\begin{array}{r}0.5324 \\
.4563 \\
.6886 \\
.5486 \\
.6253\end{array}$ \\
\hline $\begin{array}{l}134 \\
135 \\
136 \\
137 \\
138\end{array}$ & $\begin{array}{l}24.82 \\
25.44 \\
18.06 \\
14.89 \\
14.10\end{array}$ & $\begin{array}{l}34.24 \\
31.22 \\
24.04 \\
21.10 \\
20.54\end{array}$ & $\begin{array}{r}9.11 \\
20.04 \\
8.31 \\
8.40 \\
7.49\end{array}$ & $\begin{array}{r}4.00 \\
-.10 \\
-1.04 \\
-2.49 \\
-1.90\end{array}$ & $\begin{array}{r}9.40 \\
\times 4.21 \\
3.04 \\
1.89 \\
2.69\end{array}$ & $\begin{array}{l}-2.53 \\
-5.45 \\
-7.12 \\
-6.80 \\
-6.34\end{array}$ & $\begin{array}{l}.8423 \\
.6211 \\
.5790 \\
.5218 \\
.5452\end{array}$ & $\begin{array}{r}1.1785 \\
.8251 \\
.7596 \\
.7001 \\
.7410\end{array}$ & $\begin{array}{l}.5073 \\
.4075 \\
.3585 \\
.3675 \\
.3808\end{array}$ \\
\hline $\begin{array}{l}139 \\
140 \\
141 \\
142 \\
143\end{array}$ & $\begin{array}{l}13.78 \\
14.41 \\
17.16 \\
19.43 \\
21.08\end{array}$ & $\begin{array}{l}22.21 \\
25.44 \\
29.57 \\
31.49 \\
33.10\end{array}$ & $\begin{array}{l}6.25 \\
2.01 \\
2.22 \\
5.33 \\
7.75\end{array}$ & $\begin{array}{l}-1.05 \\
-2.91 \\
-5.00 \\
-5.22 \\
-2.19\end{array}$ & $\begin{array}{l}4.66 \\
4.69 \\
2.08 \\
1.44 \\
4.15\end{array}$ & $\begin{array}{r}-7.42 \\
-11.67 \\
-11.83 \\
-12.67 \\
-9.95\end{array}$ & $\begin{array}{l}.5916 \\
.5390 \\
.4537 \\
.4448 \\
.5551\end{array}$ & $\begin{array}{l}.8517 \\
.8532 \\
.7095 \\
.6808 \\
.8225\end{array}$ & $\begin{array}{l}.3503 \\
.2546 \\
.2478 \\
.2314 \\
.2876\end{array}$ \\
\hline $\begin{array}{l}144 \\
145 \\
146 \\
147 \\
148\end{array}$ & $\begin{array}{l}23.28 \\
24.97 \\
24.53 \\
25.61 \\
25.24\end{array}$ & $\begin{array}{l}33.69 \\
32.78 \\
32.00 \\
30.92 \\
33.70\end{array}$ & $\begin{array}{r}9.14 \\
15.86 \\
12.59 \\
18.69 \\
16.60\end{array}$ & $\begin{array}{r}.62 \\
6.10 \\
8.57 \\
4.16 \\
1.69\end{array}$ & $\begin{array}{r}5.18 \\
10.96 \\
13.43 \\
7.87 \\
7.04\end{array}$ & $\begin{array}{r}-6.92 \\
1.04 \\
2.64 \\
.09 \\
-6.75\end{array}$ & $\begin{array}{r}.6660 \\
.9633 \\
1.1453 \\
.8322 \\
.7220\end{array}$ & $\begin{array}{r}.8839 \\
1.3084 \\
1.5390 \\
1.0624 \\
1.0038\end{array}$ & $\begin{array}{l}.3641 \\
.6587 \\
.7386 \\
.6152 \\
.3693\end{array}$ \\
\hline $\begin{array}{l}149 \\
150 \\
151 \\
152 \\
153\end{array}$ & $\begin{array}{l}24.87 \\
25.82 \\
21.55 \\
22.89 \\
25.73\end{array}$ & $\begin{array}{l}35.80 \\
35.36 \\
30.86 \\
34.26 \\
36.95\end{array}$ & $\begin{array}{r}9.17 \\
14.28 \\
12.75 \\
10.69 \\
10.14\end{array}$ & $\begin{array}{r}-2.42 \\
.40 \\
7.31 \\
5.14 \\
3.17\end{array}$ & $\begin{array}{r}4.48 \\
4.22 \\
11.94 \\
11.33 \\
9.88\end{array}$ & $\begin{array}{r}-10.12 \\
-7.15 \\
1.07 \\
-.87 \\
-3.31\end{array}$ & $\begin{array}{r}.5470 \\
.6524 \\
1.0544 \\
.9223 \\
.8112\end{array}$ & $\begin{array}{r}.8408 \\
.8258 \\
1.3964 \\
1.3403 \\
1.2176\end{array}$ & $\begin{array}{l}.2838 \\
.3580 \\
.6597 \\
.5733 \\
.4793\end{array}$ \\
\hline $\begin{array}{l}154 \\
155 \\
156 \\
157 \\
158\end{array}$ & $\begin{array}{l}25.91 \\
23.86 \\
25.70 \\
23.58 \\
20.77\end{array}$ & $\begin{array}{l}34.83 \\
34.14 \\
33.95 \\
31.17 \\
31.70\end{array}$ & $\begin{array}{r}10.92 \\
7.67 \\
13.55 \\
17.47 \\
7.09\end{array}$ & $\begin{array}{r}-.30 \\
-3.75 \\
-.64 \\
1.67 \\
-1.66\end{array}$ & $\begin{array}{l}6.40 \\
2.48 \\
4.16 \\
7.09 \\
3.87\end{array}$ & $\begin{array}{r}-7.42 \\
-13.31 \\
-7.15 \\
-6.09 \\
-7.92\end{array}$ & $\begin{array}{l}.6362 \\
.4971 \\
.6032 \\
.7148 \\
.5668\end{array}$ & $\begin{array}{r}.9606 \\
.7298 \\
.8222 \\
1.0071 \\
.8058\end{array}$ & $\begin{array}{l}.3502 \\
.2198 \\
.3579 \\
.3880 \\
.3371\end{array}$ \\
\hline $\begin{array}{l}159 \\
160 \\
161 \\
162 \\
163\end{array}$ & $\begin{array}{l}23.57 \\
23.93 \\
26.29 \\
28.46 \\
30.19\end{array}$ & $\begin{array}{l}34.47 \\
37.90 \\
40.07 \\
39.75 \\
38.19\end{array}$ & $\begin{array}{r}12.93 \\
8.18 \\
9.76 \\
13.05 \\
20.99\end{array}$ & $\begin{array}{r}-1.37 \\
-1.44 \\
-1.18 \\
.88 \\
2.15\end{array}$ & $\begin{array}{l}3.40 \\
5.08 \\
4.84 \\
7.47 \\
7.15\end{array}$ & $\begin{array}{l}-7.44 \\
-8.94 \\
-8.89 \\
-6.44 \\
-3.25\end{array}$ & $\begin{array}{l}.5754 \\
.5874 \\
.5948 \\
.6884 \\
.7365\end{array}$ & $\begin{array}{r}.7790 \\
1.0581 \\
.8622 \\
1.0338 \\
1.0118\end{array}$ & $\begin{array}{l}.3497 \\
.3112 \\
.3126 \\
.3779 \\
.4810\end{array}$ \\
\hline $\begin{array}{l}164 \\
165 \\
166 \\
167 \\
168\end{array}$ & $\begin{array}{l}29.90 \\
28.35 \\
25.26 \\
22.94 \\
26.59\end{array}$ & $\begin{array}{l}36.55 \\
35.55 \\
32.64 \\
31.87 \\
35.07\end{array}$ & $\begin{array}{l}21.38 \\
21.90 \\
17.85 \\
10.43 \\
14.00\end{array}$ & $\begin{array}{r}1.83 \\
3.80 \\
.37 \\
-2.85 \\
-2.61\end{array}$ & $\begin{array}{l}6.60 \\
9.33 \\
5.23 \\
2.53 \\
2.64\end{array}$ & $\begin{array}{l}-2.75 \\
-2.06 \\
-6.18 \\
-9.37 \\
-7.56\end{array}$ & $\begin{array}{l}.7144 \\
.8309 \\
.6460 \\
.5195 \\
.5226\end{array}$ & $\begin{array}{r}.9738 \\
1.1733 \\
.8860 \\
.7328 \\
.7387\end{array}$ & $\begin{array}{l}.4992 \\
.5253 \\
.3855 \\
.3010 \\
.3466\end{array}$ \\
\hline $\begin{array}{l}169 \\
170 \\
171 \\
172 \\
173\end{array}$ & $\begin{array}{l}26.48 \\
26.00 \\
27.28 \\
29.95 \\
30.51\end{array}$ & $\begin{array}{l}35.91 \\
37.33 \\
37.80 \\
38.07 \\
38.65\end{array}$ & $\begin{array}{r}15.50 \\
9.17 \\
9.38 \\
19.89 \\
18.07\end{array}$ & $\begin{array}{r}-2.42 \\
-2.13 \\
-.98 \\
4.77 \\
2.56\end{array}$ & $\begin{array}{l}3.76 \\
4.68 \\
4.28 \\
9.47 \\
7.68\end{array}$ & $\begin{array}{r}-8.70 \\
-9.45 \\
-10.10 \\
-.90 \\
-3.66\end{array}$ & $\begin{array}{l}.5387 \\
.5596 \\
.6034 \\
.8776 \\
.7626\end{array}$ & $\begin{array}{r}.7993 \\
.8529 \\
.8288 \\
1.1838 \\
1.0489\end{array}$ & $\begin{array}{l}.3173 \\
.2992 \\
.2841 \\
.5722 \\
.4663\end{array}$ \\
\hline $\begin{array}{l}174 \\
175 \\
176 \\
177 \\
178\end{array}$ & $\begin{array}{l}30.33 \\
28.68 \\
32.42 \\
34.75 \\
32.86\end{array}$ & $\begin{array}{l}40.16 \\
41.05 \\
41.29 \\
42.55 \\
41.66\end{array}$ & $\begin{array}{r}15.60 \\
9.84 \\
16.22 \\
27.78 \\
22.09\end{array}$ & $\begin{array}{r}-.32 \\
.08 \\
1.50 \\
2.70 \\
5.85\end{array}$ & $\begin{array}{r}5.55 \\
6.25 \\
5.84 \\
6.49 \\
10.02\end{array}$ & $\begin{array}{r}-7.39 \\
-9.36 \\
-5.63 \\
-1.01 \\
-.22\end{array}$ & $\begin{array}{l}.6243 \\
.6588 \\
.7026 \\
.7548 \\
.9509\end{array}$ & $\begin{array}{r}.9056 \\
.9510 \\
.9243 \\
.9667 \\
1.2290\end{array}$ & $\begin{array}{l}.3516 \\
.3013 \\
.4019 \\
.5672 \\
.6012\end{array}$ \\
\hline
\end{tabular}


Table 2. Daily mean, maximum, and minimum air and dew-point temperature and vapor pressure measured by energybudget station at Rogers Spring Slte 1, 1994-Continued

\begin{tabular}{|c|c|c|c|c|c|c|c|c|c|}
\hline \multirow{2}{*}{$\begin{array}{l}\text { Calendar } \\
\text { day }\end{array}$} & \multicolumn{3}{|c|}{$\begin{array}{c}\text { Air temperature at } \\
0.7 \text { meter, in degrees Celsius }\end{array}$} & \multicolumn{3}{|c|}{$\begin{array}{l}\text { Dew-point temperature at } \\
0.7 \text { meter, in degrees Celslus }\end{array}$} & \multicolumn{3}{|c|}{$\begin{array}{c}\text { Vapor pressure at } 0.7 \text { meter, } \\
\text { In kilopascals }\end{array}$} \\
\hline & Mean & Maximum & Minimum & Mean & Maximum & Minimum & Mean & Maximum & Minimum \\
\hline $\begin{array}{l}179 \\
180 \\
181 \\
182 \\
183\end{array}$ & $\begin{array}{l}29.96 \\
32.75 \\
32.74 \\
32.65 \\
31.89\end{array}$ & $\begin{array}{l}43.10 \\
43.30 \\
43.41 \\
38.63 \\
39.66\end{array}$ & $\begin{array}{l}16.22 \\
17.97 \\
17.86 \\
24.79 \\
21.01\end{array}$ & $\begin{array}{l}5.46 \\
5.13 \\
4.18 \\
6.47 \\
5.15\end{array}$ & $\begin{array}{r}12.09 \\
10.65 \\
8.79 \\
10.35 \\
10.41\end{array}$ & $\begin{array}{r}0.88 \\
-.28 \\
-1.78 \\
1.66 \\
-1.74\end{array}$ & $\begin{array}{r}0.9300 \\
.9080 \\
.8457 \\
.9828 \\
.9106\end{array}$ & $\begin{array}{l}1.4433 \\
1.2812 \\
1.1308 \\
1.2565 \\
1.2610\end{array}$ & $\begin{array}{r}0.6510 \\
.5987 \\
.5361 \\
.6883 \\
.5379\end{array}$ \\
\hline $\begin{array}{l}184 \\
185 \\
186 \\
187 \\
188\end{array}$ & $\begin{array}{l}27.71 \\
28.68 \\
28.20 \\
26.42 \\
28.55\end{array}$ & $\begin{array}{l}37.46 \\
36.60 \\
36.16 \\
38.00 \\
38.22\end{array}$ & $\begin{array}{l}10.69 \\
18.62 \\
17.17 \\
12.50 \\
16.66\end{array}$ & $\begin{array}{l}2.62 \\
8.10 \\
7.92 \\
1.72 \\
-.51\end{array}$ & $\begin{array}{r}8.49 \\
13.04 \\
12.39 \\
10.72 \\
3.88\end{array}$ & $\begin{array}{r}-7.17 \\
2.01 \\
2.35 \\
-8.98 \\
-8.33\end{array}$ & $\begin{array}{r}.7812 \\
1.1142 \\
1.0939 \\
.7474 \\
.6085\end{array}$ & $\begin{array}{r}1.1081 \\
1.5009 \\
1.4377 \\
1.2875 \\
.8062\end{array}$ & $\begin{array}{l}.3571 \\
.7061 \\
.7233 \\
.3104 \\
.3264\end{array}$ \\
\hline $\begin{array}{l}189 \\
190 \\
191 \\
192 \\
193\end{array}$ & $\begin{array}{l}26.81 \\
29.99 \\
31.62 \\
28.63 \\
29.02\end{array}$ & $\begin{array}{l}40.51 \\
40.69 \\
39.38 \\
39.14 \\
40.72\end{array}$ & $\begin{array}{l}11.44 \\
12.65 \\
21.32 \\
14.15 \\
12.32\end{array}$ & $\begin{array}{r}-.03 \\
.61 \\
1.81 \\
2.20 \\
3.28\end{array}$ & $\begin{array}{l}5.91 \\
6.81 \\
6.44 \\
7.70 \\
8.29\end{array}$ & $\begin{array}{l}-7.34 \\
-6.08 \\
-3.69 \\
-4.50 \\
-3.51\end{array}$ & $\begin{array}{l}.6431 \\
.6702 \\
.7152 \\
.7477 \\
.8062\end{array}$ & $\begin{array}{r}.9289 \\
.9883 \\
.9637 \\
1.0504 \\
1.0934\end{array}$ & $\begin{array}{l}.3526 \\
.3883 \\
.4652 \\
.4376 \\
.4715\end{array}$ \\
\hline $\begin{array}{l}194 \\
195 \\
196 \\
197 \\
198\end{array}$ & $\begin{array}{l}29.50 \\
30.20 \\
30.24 \\
30.14 \\
30.97\end{array}$ & $\begin{array}{l}40.23 \\
40.06 \\
39.26 \\
38.81 \\
39.28\end{array}$ & $\begin{array}{l}17.74 \\
14.25 \\
18.18 \\
17.25 \\
22.45\end{array}$ & $\begin{array}{r}2.81 \\
3.76 \\
5.21 \\
8.43 \\
19.01\end{array}$ & $\begin{array}{r}8.24 \\
9.91 \\
10.58 \\
15.45 \\
22.33\end{array}$ & $\begin{array}{r}-4.27 \\
-3.09 \\
-1.10 \\
-.95 \\
13.76\end{array}$ & $\begin{array}{r}.7765 \\
.8355 \\
.9193 \\
1.1700 \\
2.2376\end{array}$ & $\begin{array}{l}1.0899 \\
1.2193 \\
1.2756 \\
1.7541 \\
2.6961\end{array}$ & $\begin{array}{r}.4454 \\
.4875 \\
.5635 \\
.5698 \\
1.5725\end{array}$ \\
\hline $\begin{array}{l}199 \\
200 \\
201 \\
202 \\
203\end{array}$ & $\begin{array}{l}31.32 \\
28.66 \\
27.88 \\
29.10 \\
31.02\end{array}$ & $\begin{array}{l}37.45 \\
36.49 \\
36.96 \\
37.66 \\
38.82\end{array}$ & $\begin{array}{l}23.69 \\
20.54 \\
18.13 \\
17.59 \\
21.74\end{array}$ & $\begin{array}{r}23.93 \\
25.11 \\
14.51 \\
11.33 \\
8.84\end{array}$ & $\begin{array}{l}26.05 \\
27.91 \\
24.49 \\
16.49 \\
13.30\end{array}$ & $\begin{array}{r}21.80 \\
21.13 \\
7.65 \\
5.90 \\
4.77\end{array}$ & $\begin{array}{l}2.9812 \\
3.2043 \\
1.7208 \\
1.3820 \\
1.1565\end{array}$ & $\begin{array}{l}3.3713 \\
3.7596 \\
3.0715 \\
1.8743 \\
1.5267\end{array}$ & $\begin{array}{r}2.6102 \\
2.5059 \\
1.0467 \\
.9279 \\
.8579\end{array}$ \\
\hline $\begin{array}{l}204 \\
205 \\
206 \\
207 \\
208\end{array}$ & $\begin{array}{l}30.27 \\
30.88 \\
29.98 \\
28.53 \\
30.21\end{array}$ & $\begin{array}{l}39.27 \\
39.50 \\
40.53 \\
41.16 \\
41.62\end{array}$ & $\begin{array}{l}16.47 \\
21.61 \\
14.63 \\
14.00 \\
13.96\end{array}$ & $\begin{array}{r}8.02 \\
11.24 \\
24.45 \\
25.44 \\
26.90\end{array}$ & $\begin{array}{l}12.75 \\
24.12 \\
29.61 \\
32.13 \\
33.63\end{array}$ & $\begin{array}{r}3.53 \\
3.53 \\
15.18 \\
14.39 \\
14.27\end{array}$ & $\begin{array}{l}1.1011 \\
1.4437 \\
3.1463 \\
3.4194 \\
3.7638\end{array}$ & $\begin{array}{l}1.4725 \\
3.0061 \\
4.1489 \\
4.7891 \\
5.2114\end{array}$ & $\begin{array}{r}.7866 \\
.7867 \\
1.7242 \\
1.6397 \\
1.6253\end{array}$ \\
\hline $\begin{array}{l}209 \\
210 \\
211 \\
212 \\
213\end{array}$ & $\begin{array}{l}31.14 \\
32.34 \\
32.68 \\
31.48 \\
32.05\end{array}$ & $\begin{array}{l}41.53 \\
40.03 \\
39.34 \\
39.68 \\
39.98\end{array}$ & $\begin{array}{l}18.14 \\
20.99 \\
26.61 \\
22.76 \\
21.71\end{array}$ & $\begin{array}{r}28.57 \\
18.95 \\
9.01 \\
4.64 \\
2.91\end{array}$ & $\begin{array}{r}34.68 \\
32.89 \\
12.60 \\
9.43 \\
7.46\end{array}$ & $\begin{array}{r}19.61 \\
7.99 \\
3.24 \\
-.32 \\
-2.36\end{array}$ & $\begin{array}{r}4.0603 \\
2.5437 \\
1.1681 \\
.8727 \\
.7724\end{array}$ & $\begin{array}{l}5.5250 \\
4.9986 \\
1.4583 \\
1.1809 \\
1.0334\end{array}$ & $\begin{array}{r}2.2817 \\
1.0714 \\
.7707 \\
.5967 \\
.5139\end{array}$ \\
\hline $\begin{array}{l}214 \\
215 \\
216 \\
217 \\
218\end{array}$ & $\begin{array}{l}30.97 \\
27.27 \\
26.77 \\
30.60 \\
33.27\end{array}$ & $\begin{array}{l}40.19 \\
40.53 \\
40.37 \\
41.84 \\
43.58\end{array}$ & $\begin{array}{r}18.42 \\
11.39 \\
9.91 \\
13.66 \\
18.88\end{array}$ & $\begin{array}{r}.10 \\
-2.15 \\
-3.39 \\
-.71 \\
3.61\end{array}$ & $\begin{array}{l}5.42 \\
3.50 \\
4.65 \\
5.20 \\
8.97\end{array}$ & $\begin{array}{r}-4.93 \\
-7.54 \\
-10.83 \\
-10.27 \\
-3.87\end{array}$ & $\begin{array}{l}.6355 \\
.5441 \\
.5190 \\
.6171 \\
.8184\end{array}$ & $\begin{array}{r}.8977 \\
.7851 \\
.8510 \\
.8839 \\
1.1451\end{array}$ & $\begin{array}{l}.4237 \\
.3472 \\
.2681 \\
.2803 \\
.4590\end{array}$ \\
\hline $\begin{array}{l}219 \\
220 \\
221 \\
222 \\
223\end{array}$ & $\begin{array}{l}32.35 \\
31.77 \\
31.02 \\
30.30 \\
31.45\end{array}$ & $\begin{array}{l}41.50 \\
36.52 \\
37.46 \\
38.30 \\
40.38\end{array}$ & $\begin{array}{l}16.98 \\
27.40 \\
25.20 \\
21.76 \\
21.89\end{array}$ & $\begin{array}{r}5.18 \\
7.64 \\
13.03 \\
14.63 \\
15.29\end{array}$ & $\begin{array}{r}9.96 \\
11.64 \\
16.76 \\
19.26 \\
22.86\end{array}$ & $\begin{array}{r}-1.76 \\
3.18 \\
9.06 \\
9.33 \\
11.21\end{array}$ & $\begin{array}{r}.9107 \\
1.0660 \\
1.5191 \\
1.6972 \\
1.7691\end{array}$ & $\begin{array}{l}1.2235 \\
1.3690 \\
1.9069 \\
2.2319 \\
2.7843\end{array}$ & $\begin{array}{r}.5368 \\
.7675 \\
1.1520 \\
1.1731 \\
1.3298\end{array}$ \\
\hline $\begin{array}{l}224 \\
225 \\
226 \\
227 \\
228\end{array}$ & $\begin{array}{l}32.64 \\
30.79 \\
29.45 \\
29.89 \\
33.32\end{array}$ & $\begin{array}{l}39.18 \\
39.31 \\
40.03 \\
42.16 \\
42.18\end{array}$ & $\begin{array}{l}25.53 \\
24.30 \\
18.91 \\
16.79 \\
21.12\end{array}$ & $\begin{array}{r}26.68 \\
28.22 \\
26.38 \\
16.42 \\
8.46\end{array}$ & $\begin{array}{l}29.66 \\
30.30 \\
31.59 \\
29.58 \\
12.85\end{array}$ & $\begin{array}{r}22.96 \\
25.16 \\
19.85 \\
4.01 \\
2.53\end{array}$ & $\begin{array}{l}3.5335 \\
3.8434 \\
3.5169 \\
2.1122 \\
1.1312\end{array}$ & $\begin{array}{l}4.1616 \\
4.3163 \\
4.6461 \\
4.1416 \\
1.4821\end{array}$ & $\begin{array}{r}2.8014 \\
3.1965 \\
2.3157 \\
.8133 \\
.7326\end{array}$ \\
\hline
\end{tabular}


Table 2. Daily mean, maximum, and minimum air and dew-point temperature and vapor pressure measured by energybudget station at Rogers Spring Site 1, 1994-Continued

\begin{tabular}{|c|c|c|c|c|c|c|c|c|c|}
\hline \multirow{2}{*}{$\begin{array}{l}\text { Calendar } \\
\text { day }\end{array}$} & \multicolumn{3}{|c|}{$\begin{array}{c}\text { Alr temperature at } \\
0.7 \text { meter, in degrees Celsius }\end{array}$} & \multicolumn{3}{|c|}{$\begin{array}{l}\text { Dew-point temperature at } \\
0.7 \text { meter, in degrees Celsius }\end{array}$} & \multicolumn{3}{|c|}{$\begin{array}{l}\text { Vapor pressure at } 0.7 \text { meter, } \\
\text { in kilopascals }\end{array}$} \\
\hline & Mean & Maximum & Minimum & Mean & Maximum & Minimum & Mean & Maximum & Minimum \\
\hline $\begin{array}{l}229 \\
230 \\
231 \\
232 \\
233\end{array}$ & $\begin{array}{l}30.52 \\
31.50 \\
30.73 \\
30.63 \\
30.25\end{array}$ & $\begin{array}{l}37.60 \\
37.72 \\
38.28 \\
39.26 \\
37.46\end{array}$ & $\begin{array}{l}22.45 \\
24.46 \\
20.38 \\
17.81 \\
21.71\end{array}$ & $\begin{array}{r}10.58 \\
11.56 \\
11.21 \\
10.01 \\
2.57\end{array}$ & $\begin{array}{r}14.93 \\
15.04 \\
14.97 \\
14.83 \\
7.49\end{array}$ & $\begin{array}{r}4.41 \\
9.54 \\
7.80 \\
4.05 \\
-3.91\end{array}$ & $\begin{array}{l}1.3055 \\
1.3683 \\
1.3469 \\
1.2545 \\
.7570\end{array}$ & $\begin{array}{l}1.8074 \\
1.7091 \\
1.7010 \\
1.6855 \\
1.0356\end{array}$ & $\begin{array}{r}0.8368 \\
1.1897 \\
1.0579 \\
.8156 \\
.4576\end{array}$ \\
\hline $\begin{array}{l}234 \\
235 \\
236 \\
237 \\
238\end{array}$ & $\begin{array}{l}27.98 \\
24.21 \\
26.41 \\
30.34 \\
30.03\end{array}$ & $\begin{array}{l}36.10 \\
37.43 \\
39.81 \\
39.18 \\
39.13\end{array}$ & $\begin{array}{l}20.24 \\
10.01 \\
10.11 \\
19.72 \\
17.35\end{array}$ & $\begin{array}{r}-.98 \\
-5.64 \\
-3.80 \\
-1.37 \\
2.58\end{array}$ & $\begin{array}{l}6.06 \\
1.43 \\
1.82 \\
2.75 \\
9.58\end{array}$ & $\begin{array}{r}-10.24 \\
-11.63 \\
-9.75 \\
-5.90 \\
-4.82\end{array}$ & $\begin{array}{l}.6063 \\
.4234 \\
.4796 \\
.5645 \\
.7712\end{array}$ & $\begin{array}{r}1.0858 \\
.6776 \\
.6965 \\
.7443 \\
1.1934\end{array}$ & $\begin{array}{l}.2811 \\
.2515 \\
.2920 \\
.3938 \\
.4274\end{array}$ \\
\hline $\begin{array}{l}239 \\
240 \\
241 \\
242 \\
243\end{array}$ & $\begin{array}{l}29.08 \\
30.07 \\
27.29 \\
23.02 \\
25.19\end{array}$ & $\begin{array}{l}36.95 \\
38.54 \\
38.08 \\
37.46 \\
36.31\end{array}$ & $\begin{array}{r}23.20 \\
20.63 \\
12.08 \\
7.52 \\
9.08\end{array}$ & $\begin{array}{r}10.35 \\
6.07 \\
-1.16 \\
-3.99 \\
-2.88\end{array}$ & $\begin{array}{r}16.59 \\
10.09 \\
4.59 \\
2.49 \\
3.84\end{array}$ & $\begin{array}{r}3.72 \\
.54 \\
-9.52 \\
-9.51 \\
-7.99\end{array}$ & $\begin{array}{r}1.2992 \\
.9601 \\
.5895 \\
.4755 \\
.5149\end{array}$ & $\begin{array}{r}1.8870 \\
1.2346 \\
.9530 \\
.7303 \\
.8040\end{array}$ & $\begin{array}{l}.7972 \\
.6350 \\
.2975 \\
.2977 \\
.3353\end{array}$ \\
\hline $\begin{array}{l}244 \\
245 \\
246 \\
247 \\
248\end{array}$ & $\begin{array}{l}26.95 \\
27.09 \\
22.72 \\
22.62 \\
25.28\end{array}$ & $\begin{array}{l}35.57 \\
34.97 \\
34.30 \\
36.01 \\
37.27\end{array}$ & $\begin{array}{r}16.33 \\
13.74 \\
9.28 \\
8.04 \\
12.93\end{array}$ & $\begin{array}{r}-1.55 \\
-2.58 \\
-2.72 \\
1.61 \\
6.82\end{array}$ & $\begin{array}{r}4.35 \\
1.27 \\
2.25 \\
8.62 \\
12.12\end{array}$ & $\begin{array}{l}-6.45 \\
-5.92 \\
-8.54 \\
-5.69 \\
0\end{array}$ & $\begin{array}{r}.5641 \\
.5119 \\
.5160 \\
.7253 \\
1.0249\end{array}$ & $\begin{array}{r}.8330 \\
.6698 \\
.7182 \\
1.1181 \\
1.4130\end{array}$ & $\begin{array}{l}.3774 \\
.3932 \\
.3213 \\
.4001 \\
.6106\end{array}$ \\
\hline $\begin{array}{l}249 \\
250 \\
251 \\
252 \\
253\end{array}$ & $\begin{array}{l}28.33 \\
28.98 \\
29.24 \\
27.74 \\
29.00\end{array}$ & $\begin{array}{l}37.86 \\
38.15 \\
37.07 \\
34.82 \\
34.84\end{array}$ & $\begin{array}{l}15.99 \\
20.08 \\
17.80 \\
13.39 \\
23.96\end{array}$ & $\begin{array}{r}7.70 \\
3.17 \\
.49 \\
-1.13 \\
-2.07\end{array}$ & $\begin{array}{r}13.55 \\
8.92 \\
6.30 \\
2.05 \\
1.02\end{array}$ & $\begin{array}{l}3.31 \\
-2.58 \\
-5.66 \\
-8.06 \\
-5.83\end{array}$ & $\begin{array}{r}1.0790 \\
.7884 \\
.6558 \\
.5812 \\
.5309\end{array}$ & $\begin{array}{r}1.5514 \\
1.1413 \\
.9540 \\
.7082 \\
.6576\end{array}$ & $\begin{array}{l}.7744 \\
.5053 \\
.4009 \\
.3334 \\
.3957\end{array}$ \\
\hline $\begin{array}{l}254 \\
255 \\
256 \\
257 \\
258\end{array}$ & $\begin{array}{l}25.03 \\
21.60 \\
15.87 \\
18.19 \\
20.38\end{array}$ & $\begin{array}{l}31.39 \\
27.01 \\
25.80 \\
29.92 \\
33.25\end{array}$ & $\begin{array}{r}19.07 \\
16.04 \\
5.68 \\
8.48 \\
6.86\end{array}$ & $\begin{array}{r}-3.36 \\
-1.73 \\
-1.82 \\
-.87 \\
-2.28\end{array}$ & $\begin{array}{r}-2.16 \\
3.67 \\
3.81 \\
4.95 \\
3.39\end{array}$ & $\begin{array}{l}-5.24 \\
-4.65 \\
-7.22 \\
-6.16 \\
-7.90\end{array}$ & $\begin{array}{l}.4784 \\
.5486 \\
.5547 \\
.5952 \\
.5377\end{array}$ & $\begin{array}{l}.5215 \\
.8928 \\
.8021 \\
.8691 \\
.7785\end{array}$ & $\begin{array}{l}.4141 \\
.4330 \\
.3559 \\
.3859 \\
.3377\end{array}$ \\
\hline $\begin{array}{l}259 \\
260 \\
261 \\
262 \\
263\end{array}$ & $\begin{array}{l}20.51 \\
20.79 \\
21.28 \\
21.24 \\
23.34\end{array}$ & $\begin{array}{l}33.14 \\
33.97 \\
34.49 \\
30.07 \\
32.18\end{array}$ & $\begin{array}{r}6.05 \\
8.41 \\
9.75 \\
12.28 \\
14.35\end{array}$ & $\begin{array}{r}-2.06 \\
-1.95 \\
.95 \\
4.81 \\
6.92\end{array}$ & $\begin{array}{r}3.46 \\
3.27 \\
6.24 \\
10.87 \\
11.82\end{array}$ & $\begin{array}{r}-7.44 \\
-8.02 \\
-4.61 \\
-1.33 \\
3.58\end{array}$ & $\begin{array}{r}.5563 \\
.5504 \\
.6756 \\
.8907 \\
1.0167\end{array}$ & $\begin{array}{r}1.0909 \\
.7719 \\
.9501 \\
1.3007 \\
1.3850\end{array}$ & $\begin{array}{l}.3499 \\
.3343 \\
.4342 \\
.5543 \\
.7895\end{array}$ \\
\hline $\begin{array}{l}264 \\
265 \\
266 \\
267 \\
268\end{array}$ & $\begin{array}{l}22.34 \\
21.35 \\
21.66 \\
23.82 \\
20.53\end{array}$ & $\begin{array}{l}33.58 \\
34.74 \\
35.18 \\
32.50 \\
33.92\end{array}$ & $\begin{array}{r}12.84 \\
8.00 \\
7.42 \\
14.66 \\
9.22\end{array}$ & $\begin{array}{r}3.76 \\
1.90 \\
.42 \\
5.87 \\
2.59\end{array}$ & $\begin{array}{r}8.81 \\
7.90 \\
5.96 \\
11.95 \\
9.13\end{array}$ & $\begin{array}{r}-1.15 \\
-2.78 \\
-5.54 \\
1.05 \\
-2.83\end{array}$ & $\begin{array}{l}.8192 \\
.7258 \\
.6522 \\
.9507 \\
.7678\end{array}$ & $\begin{array}{r}1.1341 \\
1.0646 \\
.9319 \\
1.3975 \\
1.1575\end{array}$ & $\begin{array}{l}.5618 \\
.4979 \\
.4047 \\
.6594 \\
.4964\end{array}$ \\
\hline $\begin{array}{l}269 \\
270 \\
271 \\
272 \\
273\end{array}$ & $\begin{array}{l}20.56 \\
22.77 \\
24.72 \\
22.09 \\
18.04\end{array}$ & $\begin{array}{l}34.98 \\
35.18 \\
32.62 \\
30.35 \\
28.00\end{array}$ & $\begin{array}{r}7.50 \\
7.98 \\
12.15 \\
15.06 \\
8.03\end{array}$ & $\begin{array}{l}.15 \\
2.12 \\
7.28 \\
8.19 \\
2.35\end{array}$ & $\begin{array}{r}5.57 \\
7.10 \\
11.61 \\
14.12 \\
8.17\end{array}$ & $\begin{array}{r}-5.32 \\
-5.58 \\
1.06 \\
.94 \\
-4.04\end{array}$ & $\begin{array}{r}.6407 \\
.7433 \\
1.0422 \\
1.1306 \\
.7475\end{array}$ & $\begin{array}{r}.9072 \\
1.0083 \\
1.3657 \\
1.6100 \\
1.0849\end{array}$ & $\begin{array}{l}.4114 \\
.4034 \\
.6595 \\
.6539 \\
.4532\end{array}$ \\
\hline $\begin{array}{l}274 \\
275 \\
276 \\
277 \\
278\end{array}$ & $\begin{array}{l}17.68 \\
20.58 \\
18.52 \\
18.24 \\
13.97\end{array}$ & $\begin{array}{l}29.52 \\
31.39 \\
22.97 \\
21.53 \\
21.06\end{array}$ & $\begin{array}{r}5.90 \\
7.64 \\
9.89 \\
15.31 \\
3.78\end{array}$ & $\begin{array}{r}2.12 \\
2.59 \\
.27 \\
4.12 \\
8.18\end{array}$ & $\begin{array}{r}8.31 \\
8.28 \\
2.39 \\
6.85 \\
26.90\end{array}$ & $\begin{array}{l}-4.18 \\
-2.67 \\
-3.65 \\
1.49 \\
2.91\end{array}$ & $\begin{array}{r}.7461 \\
.7596 \\
.6305 \\
.8246 \\
1.2045\end{array}$ & $\begin{array}{r}1.0950 \\
1.0930 \\
.7258 \\
.9909 \\
3.5453\end{array}$ & $\begin{array}{l}.4485 \\
.5020 \\
.4669 \\
.6802 \\
.7528\end{array}$ \\
\hline
\end{tabular}


Table 2. Daily mean, maximum, and minimum air and dew-point temperature and vapor pressure measured by energybudget station at Rogers Spring Slte 1, 1994-Continued

\begin{tabular}{|c|c|c|c|c|c|c|c|c|c|}
\hline \multirow{2}{*}{$\begin{array}{l}\text { Calendar } \\
\text { day }\end{array}$} & \multicolumn{3}{|c|}{$\begin{array}{c}\text { Air temperature at } \\
0.7 \text { meter, in degrees Celsius }\end{array}$} & \multicolumn{3}{|c|}{$\begin{array}{c}\text { Dew-point temperature at } \\
0.7 \text { meter, in degrees Celsius }\end{array}$} & \multicolumn{3}{|c|}{$\begin{array}{l}\text { Vapor pressure at } 0.7 \text { meter, } \\
\text { in kilopascals }\end{array}$} \\
\hline & Mean & Maximum & Minimum & Mean & Maximum & Minimum & Mean & Maximum & Minimum \\
\hline $\begin{array}{l}279 \\
280 \\
281 \\
282 \\
283\end{array}$ & $\begin{array}{l}13.47 \\
17.36 \\
18.13 \\
15.13 \\
16.08\end{array}$ & $\begin{array}{l}24.08 \\
27.41 \\
28.22 \\
29.48 \\
29.36\end{array}$ & $\begin{array}{l}0.71 \\
5.36 \\
6.61 \\
2.23 \\
3.36\end{array}$ & $\begin{array}{r}15.27 \\
4.62 \\
-1.48 \\
-4.83 \\
-5.37\end{array}$ & $\begin{array}{r}30.07 \\
24.18 \\
3.43 \\
-1.39 \\
-1.96\end{array}$ & $\begin{array}{r}1.44 \\
-4.81 \\
-7.61 \\
-9.37 \\
-9.33\end{array}$ & $\begin{array}{r}2.0753 \\
1.0078 \\
.5668 \\
.4366 \\
.4172\end{array}$ & $\begin{array}{r}4.2596 \\
3.0195 \\
.7810 \\
.5525 \\
.5290\end{array}$ & $\begin{array}{r}0.6779 \\
.4278 \\
.3453 \\
.3014 \\
.3018\end{array}$ \\
\hline $\begin{array}{l}284 \\
285 \\
286 \\
287 \\
288\end{array}$ & $\begin{array}{l}18.14 \\
19.18 \\
14.83 \\
16.36 \\
12.48\end{array}$ & $\begin{array}{l}28.51 \\
27.66 \\
22.96 \\
21.61 \\
17.80\end{array}$ & $\begin{array}{r}5.32 \\
4.59 \\
3.94 \\
11.20 \\
5.07\end{array}$ & $\begin{array}{r}-5.32 \\
-6.87 \\
-3.01 \\
1.86 \\
-1.99\end{array}$ & $\begin{array}{r}-2.78 \\
-3.45 \\
2.91 \\
5.70 \\
1.44\end{array}$ & $\begin{array}{r}-10.15 \\
-9.16 \\
-9.05 \\
-1.08 \\
-7.75\end{array}$ & $\begin{array}{l}.4166 \\
.3687 \\
.5133 \\
.7075 \\
.5390\end{array}$ & $\begin{array}{l}.4978 \\
.4738 \\
.7527 \\
.9156 \\
.6776\end{array}$ & $\begin{array}{l}.2829 \\
.3062 \\
.3085 \\
.5647 \\
.3415\end{array}$ \\
\hline $\begin{array}{l}289 \\
290 \\
291 \\
292 \\
293\end{array}$ & $\begin{array}{r}10.65 \\
9.39 \\
10.27 \\
10.66 \\
10.93\end{array}$ & $\begin{array}{l}19.00 \\
19.79 \\
22.24 \\
23.65 \\
24.20\end{array}$ & $\begin{array}{r}1.60 \\
-.19 \\
-.64 \\
.09 \\
-.06\end{array}$ & $\begin{array}{l}-4.55 \\
-3.89 \\
-4.78 \\
-5.31 \\
-5.83\end{array}$ & $\begin{array}{r}-.07 \\
3.06 \\
1.88 \\
1.73 \\
.82\end{array}$ & $\begin{array}{r}-9.35 \\
-10.47 \\
-9.53 \\
-10.53 \\
-11.37\end{array}$ & $\begin{array}{l}.4471 \\
.4846 \\
.4493 \\
.4354 \\
.4172\end{array}$ & $\begin{array}{l}.6076 \\
.7608 \\
.6993 \\
.6919 \\
.6483\end{array}$ & $\begin{array}{l}.3018 \\
.2759 \\
.2974 \\
.2747 \\
.2569\end{array}$ \\
\hline $\begin{array}{l}294 \\
295 \\
296 \\
297 \\
298\end{array}$ & $\begin{array}{l}10.86 \\
11.13 \\
13.16 \\
14.51 \\
13.44\end{array}$ & $\begin{array}{l}25.82 \\
24.87 \\
25.30 \\
25.90 \\
26.77\end{array}$ & $\begin{array}{r}-2.36 \\
-.56 \\
-1.01 \\
4.43 \\
3.13\end{array}$ & $\begin{array}{l}-6.39 \\
-6.36 \\
-5.54 \\
-3.18 \\
-3.45\end{array}$ & $\begin{array}{r}1.26 \\
.22 \\
.32 \\
1.96 \\
3.39\end{array}$ & $\begin{array}{r}-12.18 \\
-11.93 \\
-12.49 \\
-8.82 \\
-8.36\end{array}$ & $\begin{array}{l}.4072 \\
.4025 \\
.4249 \\
.4995 \\
.5120\end{array}$ & $\begin{array}{l}.6690 \\
.6208 \\
.6250 \\
.7032 \\
.9288\end{array}$ & $\begin{array}{l}.2407 \\
.2457 \\
.2348 \\
.3142 \\
.3257\end{array}$ \\
\hline $\begin{array}{l}299 \\
300 \\
301 \\
302 \\
303\end{array}$ & $\begin{array}{l}13.19 \\
13.37 \\
12.08 \\
13.12 \\
12.72\end{array}$ & $\begin{array}{l}26.71 \\
26.45 \\
25.84 \\
25.32 \\
22.55\end{array}$ & $\begin{array}{r}1.95 \\
3.00 \\
2.00 \\
.98 \\
2.07\end{array}$ & $\begin{array}{l}-3.35 \\
-3.57 \\
-4.27 \\
-4.58 \\
-7.43\end{array}$ & $\begin{array}{r}4.31 \\
4.25 \\
3.54 \\
2.86 \\
-1.45\end{array}$ & $\begin{array}{r}-9.60 \\
-9.71 \\
-10.11 \\
-10.73 \\
-15.64\end{array}$ & $\begin{array}{l}.5094 \\
.5002 \\
.4769 \\
.4659 \\
.3685\end{array}$ & $\begin{array}{l}.8306 \\
.8276 \\
.7871 \\
.7499 \\
.5494\end{array}$ & $\begin{array}{l}.2955 \\
.2930 \\
.2840 \\
.2702 \\
.1814\end{array}$ \\
\hline $\begin{array}{l}304 \\
305 \\
306 \\
307 \\
308\end{array}$ & $\begin{array}{r}9.48 \\
14.30 \\
13.42 \\
6.51 \\
3.25\end{array}$ & $\begin{array}{l}24.20 \\
25.43 \\
17.93 \\
11.54 \\
12.04\end{array}$ & $\begin{array}{r}-2.79 \\
-.60 \\
7.28 \\
.90 \\
-3.55\end{array}$ & $\begin{array}{r}-12.03 \\
-9.91 \\
-3.48 \\
-7.22 \\
-12.22\end{array}$ & $\begin{array}{r}2.76 \\
-3.73 \\
-.93 \\
-3.70 \\
-6.36\end{array}$ & $\begin{array}{r}-17.64 \\
-18.86 \\
-6.41 \\
-13.12 \\
-16.47\end{array}$ & $\begin{array}{l}.2884 \\
.3151 \\
.4754 \\
.3657 \\
.2503\end{array}$ & $\begin{array}{r}1.0301 \\
.4639 \\
.5711 \\
.4649 \\
.3800\end{array}$ & $\begin{array}{l}.1535 \\
.1383 \\
.3788 \\
.2232 \\
.1692\end{array}$ \\
\hline $\begin{array}{l}309 \\
310 \\
311 \\
312 \\
313\end{array}$ & $\begin{array}{r}6.95 \\
10.98 \\
9.06 \\
9.13 \\
11.57\end{array}$ & $\begin{array}{l}17.47 \\
22.07 \\
18.44 \\
18.07 \\
19.42\end{array}$ & $\begin{array}{r}-2.62 \\
-.81 \\
.34 \\
1.65 \\
1.06\end{array}$ & $\begin{array}{r}-11.65 \\
-8.70 \\
-9.94 \\
-5.19 \\
-4.62\end{array}$ & $\begin{array}{r}-6.62 \\
-3.55 \\
-4.86 \\
1.65 \\
-.49\end{array}$ & $\begin{array}{r}-16.06 \\
-14.25 \\
-15.07 \\
-12.86 \\
-8.09\end{array}$ & $\begin{array}{l}.2604 \\
.3293 \\
.2962 \\
.4417 \\
.4436\end{array}$ & $\begin{array}{l}.3728 \\
.4700 \\
.4260 \\
.6882 \\
.5912\end{array}$ & $\begin{array}{l}.1751 \\
.2034 \\
.1902 \\
.2279 \\
.3328\end{array}$ \\
\hline $\begin{array}{l}314 \\
315 \\
316 \\
317 \\
318\end{array}$ & $\begin{array}{l}9.11 \\
3.93 \\
3.31 \\
6.25 \\
3.75\end{array}$ & $\begin{array}{l}14.05 \\
11.98 \\
13.25 \\
12.15 \\
12.36\end{array}$ & $\begin{array}{r}.98 \\
-6.35 \\
-6.59 \\
1.96 \\
-6.58\end{array}$ & $\begin{array}{l}-3.30 \\
-2.99 \\
-3.80 \\
-5.53 \\
-9.23\end{array}$ & $\begin{array}{r}1.70 \\
3.72 \\
4.13 \\
-1.25 \\
-3.98\end{array}$ & $\begin{array}{r}-6.24 \\
-10.55 \\
-10.47 \\
-9.85 \\
-16.23\end{array}$ & $\begin{array}{l}.4906 \\
.5138 \\
.4945 \\
.4134 \\
.3161\end{array}$ & $\begin{array}{l}.7128 \\
.7971 \\
.8204 \\
.5576 \\
.4553\end{array}$ & $\begin{array}{l}.3915 \\
.2743 \\
.2760 \\
.2899 \\
.1727\end{array}$ \\
\hline $\begin{array}{l}319 \\
320\end{array}$ & $\begin{array}{l}3.45 \\
8.08\end{array}$ & $\begin{array}{l}14.07 \\
13.06\end{array}$ & $\begin{array}{r}-11.59 \\
-2.80\end{array}$ & $\begin{array}{r}1.68 \\
10.53\end{array}$ & $\begin{array}{l}18.38 \\
18.07\end{array}$ & $\begin{array}{r}-19.29 \\
-.05\end{array}$ & $\begin{array}{l}1.0048 \\
1.3289\end{array}$ & $\begin{array}{l}2.1133 \\
2.0716\end{array}$ & $\begin{array}{l}.1334 \\
.6102\end{array}$ \\
\hline
\end{tabular}


Table 3. Daily mean, maximum, and minimum micrometeorological data measured by micromet station at Rogers Spring Site 1, 1994 [-, no data available]

\begin{tabular}{|c|c|c|c|c|c|c|c|c|c|c|c|c|}
\hline & \multirow{2}{*}{$\begin{array}{l}\text { Calendar } \\
\text { day }\end{array}$} & \multicolumn{2}{|c|}{$\begin{array}{l}\text { Net radiation, } \\
\text { in watts per } \\
\text { square meter }\end{array}$} & \multicolumn{3}{|c|}{$\begin{array}{l}\text { Air temperature at } 0.7 \text { meter, } \\
\text { in degrees Celsius }\end{array}$} & \multicolumn{3}{|c|}{$\begin{array}{l}\text { Relative humidity at } 0.7 \text { meter, } \\
\text { in percent }\end{array}$} & \multicolumn{3}{|c|}{$\begin{array}{l}\text { Windspeed at } 0.7 \text { meter, } \\
\text { in meters per second }\end{array}$} \\
\hline & & Mean & Maximum & Mean & Maximum & Minimum & Mean & Maximum & Minimum & Mean & Maximum & Minimum \\
\hline & $\begin{array}{l}1 \\
2 \\
3 \\
4 \\
5\end{array}$ & $\begin{array}{r}2.0 \\
10.9 \\
14.1 \\
15.1 \\
3.1\end{array}$ & $\begin{array}{l}134.7 \\
189.0 \\
185.6 \\
166.0 \\
182.5\end{array}$ & $\begin{array}{r}4.05 \\
5.75 \\
5.53 \\
7.54 \\
10.12\end{array}$ & $\begin{array}{l}13.93 \\
18.36 \\
18.59 \\
16.71 \\
20.30\end{array}$ & $\begin{array}{r}-2.54 \\
-4.28 \\
-4.20 \\
.06 \\
-.10\end{array}$ & $\begin{array}{l}53.5 \\
50.7 \\
49.4 \\
46.5 \\
35.8\end{array}$ & $\begin{array}{l}72.6 \\
80.9 \\
76.6 \\
71.0 \\
73.9\end{array}$ & $\begin{array}{l}25.7 \\
19.9 \\
18.3 \\
21.0 \\
15.7\end{array}$ & $\begin{array}{r}0.74 \\
.90 \\
.77 \\
.66 \\
1.73\end{array}$ & $\begin{array}{l}1.21 \\
1.59 \\
1.09 \\
1.22 \\
3.62\end{array}$ & $\begin{array}{r}0.39 \\
.35 \\
.33 \\
.34 \\
.46\end{array}$ \\
\hline & $\begin{array}{r}6 \\
7 \\
8 \\
9 \\
10\end{array}$ & $\begin{array}{r}-6.1 \\
6.7 \\
8.0 \\
-1.0 \\
6.7\end{array}$ & $\begin{array}{l}189.6 \\
165.9 \\
191.2 \\
146.6 \\
204.5\end{array}$ & $\begin{array}{r}6.72 \\
3.04 \\
.89 \\
3.02 \\
6.58\end{array}$ & $\begin{array}{l}13.82 \\
11.95 \\
13.09 \\
15.35 \\
15.42\end{array}$ & $\begin{array}{r}.76 \\
-5.78 \\
-6.89 \\
-7.06 \\
-4.32\end{array}$ & $\begin{array}{l}32.8 \\
45.9 \\
49.4 \\
47.6 \\
46.3\end{array}$ & $\begin{array}{l}50.4 \\
74.9 \\
76.1 \\
68.6 \\
76.6\end{array}$ & $\begin{array}{l}17.7 \\
19.6 \\
17.3 \\
24.6 \\
23.0\end{array}$ & $\begin{array}{r}1.83 \\
.80 \\
.80 \\
1.09 \\
1.58\end{array}$ & $\begin{array}{l}3.13 \\
1.45 \\
1.42 \\
2.58 \\
3.41\end{array}$ & $\begin{array}{r}1.01 \\
.34 \\
.32 \\
.49 \\
.52\end{array}$ \\
\hline & $\begin{array}{l}11 \\
12 \\
13 \\
14 \\
15\end{array}$ & $\begin{array}{r}5.1 \\
6.5 \\
13.4 \\
5.4 \\
16.6\end{array}$ & $\begin{array}{l}189.0 \\
196.6 \\
190.7 \\
172.7 \\
211.3\end{array}$ & $\begin{array}{r}4.97 \\
8.99 \\
10.46 \\
12.01 \\
8.26\end{array}$ & $\begin{array}{l}15.98 \\
18.76 \\
21.78 \\
20.81 \\
20.39\end{array}$ & $\begin{array}{r}-6.47 \\
.61 \\
-1.09 \\
4.64 \\
.56\end{array}$ & $\begin{array}{l}48.4 \\
37.3 \\
41.9 \\
44.0 \\
52.6\end{array}$ & $\begin{array}{l}81.0 \\
62.5 \\
65.2 \\
63.4 \\
81.4\end{array}$ & $\begin{array}{l}20.8 \\
16.6 \\
22.6 \\
23.4 \\
23.1\end{array}$ & $\begin{array}{r}1.07 \\
1.40 \\
1.57 \\
1.56 \\
.79\end{array}$ & $\begin{array}{l}2.94 \\
2.76 \\
3.28 \\
2.46 \\
1.36\end{array}$ & $\begin{array}{l}.29 \\
.61 \\
.57 \\
.40 \\
.36\end{array}$ \\
\hline & $\begin{array}{l}16 \\
17 \\
18 \\
19 \\
20\end{array}$ & $\begin{array}{r}9.0 \\
13.4 \\
10.6 \\
13.0 \\
14.2\end{array}$ & $\begin{array}{l}203.7 \\
207.1 \\
203.5 \\
201.9 \\
207.4\end{array}$ & $\begin{array}{r}8.30 \\
10.14 \\
10.76 \\
7.12 \\
6.72\end{array}$ & $\begin{array}{l}21.99 \\
22.44 \\
24.86 \\
22.26 \\
21.72\end{array}$ & $\begin{array}{r}-2.15 \\
1.98 \\
-.71 \\
-2.88 \\
-3.61\end{array}$ & $\begin{array}{l}40.7 \\
32.0 \\
32.8 \\
36.7 \\
37.5\end{array}$ & $\begin{array}{l}74.1 \\
56.5 \\
63.3 \\
68.2 \\
64.6\end{array}$ & $\begin{array}{r}12.4 \\
13.7 \\
9.0 \\
11.1 \\
12.1\end{array}$ & $\begin{array}{r}1.27 \\
1.33 \\
1.13 \\
.88 \\
.87\end{array}$ & $\begin{array}{l}2.25 \\
2.82 \\
2.05 \\
1.35 \\
1.19\end{array}$ & $\begin{array}{l}.60 \\
.59 \\
.54 \\
.33 \\
.39\end{array}$ \\
\hline & $\begin{array}{l}21 \\
22 \\
23 \\
24 \\
25\end{array}$ & $\begin{array}{r}20.8 \\
15.0 \\
-9.4 \\
13.8 \\
18.4\end{array}$ & $\begin{array}{r}216.0 \\
207.7 \\
79.0 \\
223.6 \\
281.6\end{array}$ & $\begin{array}{r}7.65 \\
8.06 \\
9.65 \\
11.95 \\
6.01\end{array}$ & $\begin{array}{l}21.81 \\
21.54 \\
17.75 \\
18.51 \\
10.21\end{array}$ & $\begin{array}{r}-3.35 \\
.17 \\
-1.82 \\
2.43 \\
-.12\end{array}$ & $\begin{array}{l}37.0 \\
37.2 \\
45.3 \\
50.1 \\
80.2\end{array}$ & $\begin{array}{l}61.6 \\
57.8 \\
67.1 \\
85.2 \\
94.3\end{array}$ & $\begin{array}{l}12.2 \\
12.7 \\
22.1 \\
22.3 \\
48.0\end{array}$ & $\begin{array}{r}.71 \\
.72 \\
2.11 \\
2.56 \\
1.97\end{array}$ & $\begin{array}{l}1.03 \\
1.30 \\
4.87 \\
4.33 \\
3.33\end{array}$ & $\begin{array}{l}.45 \\
.33 \\
.31 \\
.37 \\
.41\end{array}$ \\
\hline$\vec{m}$ & $\begin{array}{l}26 \\
27 \\
28 \\
29 \\
30\end{array}$ & $\begin{array}{l}49.3 \\
29.1 \\
18.1 \\
17.7 \\
20.1\end{array}$ & $\begin{array}{l}294.4 \\
231.6 \\
253.1 \\
255.9 \\
297.3\end{array}$ & $\begin{array}{l}3.83 \\
5.79 \\
6.76 \\
7.79 \\
7.85\end{array}$ & $\begin{array}{l}14.41 \\
12.92 \\
14.35 \\
16.44 \\
17.28\end{array}$ & $\begin{array}{r}-3.58 \\
-.68 \\
.87 \\
1.98 \\
-2.76\end{array}$ & $\begin{array}{l}70.0 \\
54.3 \\
48.0 \\
40.2 \\
37.7\end{array}$ & $\begin{array}{l}95.2 \\
84.4 \\
65.1 \\
59.4 \\
72.3\end{array}$ & $\begin{array}{l}27.9 \\
27.8 \\
28.6 \\
20.4 \\
20.3\end{array}$ & $\begin{array}{l}1.31 \\
1.47 \\
2.00 \\
2.28 \\
2.35\end{array}$ & $\begin{array}{l}4.01 \\
2.59 \\
3.21 \\
4.01 \\
4.55\end{array}$ & $\begin{array}{l}.36 \\
.40 \\
.96 \\
.45 \\
.72\end{array}$ \\
\hline $\begin{array}{l}\frac{0}{\sigma} \\
\omega \\
\vec{\sigma}\end{array}$ & $\begin{array}{l}31 \\
32 \\
33 \\
34 \\
35\end{array}$ & $\begin{array}{r}10.4 \\
-.5 \\
27.8 \\
25.0 \\
25.8\end{array}$ & $\begin{array}{l}262.4 \\
177.8 \\
238.9 \\
224.2 \\
183.8\end{array}$ & $\begin{array}{l}4.40 \\
3.07 \\
2.86 \\
6.25 \\
5.18\end{array}$ & $\begin{array}{r}10.01 \\
10.37 \\
12.20 \\
14.48 \\
8.54\end{array}$ & $\begin{array}{r}.81 \\
-1.67 \\
-9.07 \\
-3.82 \\
.88\end{array}$ & $\begin{array}{l}29.4 \\
30.7 \\
39.9 \\
39.7 \\
86.6\end{array}$ & $\begin{array}{l}40.5 \\
47.3 \\
80.1 \\
91.5 \\
95.2\end{array}$ & $\begin{array}{l}16.0 \\
14.0 \\
11.6 \\
12.4 \\
70.9\end{array}$ & $\begin{array}{r}2.40 \\
1.39 \\
.75 \\
1.01 \\
.89\end{array}$ & $\begin{array}{l}4.05 \\
3.05 \\
1.36 \\
2.54 \\
2.17\end{array}$ & $\begin{array}{l}.85 \\
.46 \\
.21 \\
.27 \\
.21\end{array}$ \\
\hline
\end{tabular}


Table 3. Daily mean, maximum, and minimum micrometeorological data measured by micromet station at Rogers Spring Site 1, 1994-Continued

\begin{tabular}{|c|c|c|c|c|c|c|c|c|c|c|c|}
\hline \multirow{2}{*}{$\begin{array}{l}\text { Calendar } \\
\text { day }\end{array}$} & \multicolumn{2}{|c|}{$\begin{array}{l}\text { Net radiation, } \\
\text { in watts per } \\
\text { square meter }\end{array}$} & \multicolumn{3}{|c|}{$\begin{array}{l}\text { Air temperature at } 0.7 \text { meter, } \\
\text { in degrees Celsius }\end{array}$} & \multicolumn{3}{|c|}{$\begin{array}{l}\text { Relative humidity at } 0.7 \text { meter, } \\
\text { in percent }\end{array}$} & \multicolumn{3}{|c|}{$\begin{array}{l}\text { Windspeed at } 0.7 \text { meter, } \\
\text { in meters per second }\end{array}$} \\
\hline & Mean & Maximum & Mean & Maximum & Minimum & Mean & Maximum & Minimum & Mean & Maximum & Minimum \\
\hline $\begin{array}{l}36 \\
37 \\
38 \\
39 \\
40\end{array}$ & $\begin{array}{l}42.1 \\
37.5 \\
29.2 \\
38.3 \\
42.8\end{array}$ & $\begin{array}{l}273.8 \\
249.2 \\
191.2 \\
341.7 \\
344.0\end{array}$ & $\begin{array}{r}6.01 \\
9.29 \\
10.01 \\
9.78 \\
7.42\end{array}$ & $\begin{array}{l}11.90 \\
14.62 \\
12.92 \\
15.16 \\
16.16\end{array}$ & $\begin{array}{r}-1.10 \\
4.89 \\
7.63 \\
4.98 \\
.16\end{array}$ & $\begin{array}{l}77.1 \\
67.9 \\
79.8 \\
67.3 \\
54.9\end{array}$ & $\begin{array}{l}96.2 \\
91.1 \\
91.5 \\
87.7 \\
87.0\end{array}$ & $\begin{array}{l}47.9 \\
44.3 \\
57.6 \\
43.1 \\
18.9\end{array}$ & $\begin{array}{l}0.67 \\
1.01 \\
2.61 \\
2.50 \\
1.45\end{array}$ & $\begin{array}{l}1.46 \\
2.37 \\
5.08 \\
4.77 \\
2.72\end{array}$ & $\begin{array}{r}0.24 \\
.24 \\
.57 \\
.47 \\
.47\end{array}$ \\
\hline $\begin{array}{l}41 \\
42 \\
43 \\
44 \\
45\end{array}$ & $\begin{array}{l}46.7 \\
33.6 \\
30.6 \\
33.4 \\
44.0\end{array}$ & $\begin{array}{l}345.2 \\
328.3 \\
330.6 \\
281.2 \\
333.9\end{array}$ & $\begin{array}{l}9.47 \\
7.63 \\
4.35 \\
2.70 \\
5.70\end{array}$ & $\begin{array}{l}18.94 \\
11.90 \\
13.40 \\
14.68 \\
18.34\end{array}$ & $\begin{array}{r}-1.25 \\
.54 \\
-3.62 \\
-8.58 \\
-3.81\end{array}$ & $\begin{array}{l}51.5 \\
34.7 \\
34.5 \\
51.3 \\
46.8\end{array}$ & $\begin{array}{l}91.5 \\
58.1 \\
68.4 \\
88.5 \\
81.0\end{array}$ & $\begin{array}{r}18.3 \\
20.5 \\
14.0 \\
15.0 \\
9.6\end{array}$ & $\begin{array}{r}2.51 \\
3.88 \\
1.77 \\
.88 \\
.81\end{array}$ & $\begin{array}{l}4.76 \\
6.61 \\
3.56 \\
2.12 \\
1.30\end{array}$ & $\begin{array}{r}.41 \\
1.17 \\
.42 \\
.32 \\
.33\end{array}$ \\
\hline $\begin{array}{l}46 \\
47 \\
48 \\
49 \\
50\end{array}$ & $\begin{array}{l}23.2 \\
30.0 \\
12.6 \\
39.3 \\
59.5\end{array}$ & $\begin{array}{l}295.3 \\
218.8 \\
229.9 \\
396.7 \\
386.3\end{array}$ & $\begin{array}{r}4.94 \\
10.20 \\
11.53 \\
8.29 \\
6.76\end{array}$ & $\begin{array}{l}16.97 \\
18.09 \\
14.83 \\
13.96 \\
12.79\end{array}$ & $\begin{array}{r}-6.23 \\
1.69 \\
6.74 \\
4.56 \\
-.88\end{array}$ & $\begin{array}{l}49.3 \\
40.7 \\
49.2 \\
43.1 \\
51.4\end{array}$ & $\begin{array}{l}83.8 \\
70.1 \\
77.7 \\
60.0 \\
83.2\end{array}$ & $\begin{array}{l}16.2 \\
18.5 \\
28.7 \\
21.9 \\
31.6\end{array}$ & $\begin{array}{r}.70 \\
1.09 \\
3.69 \\
3.16 \\
1.82\end{array}$ & $\begin{array}{l}1.29 \\
2.25 \\
6.33 \\
4.37 \\
3.06\end{array}$ & $\begin{array}{r}.29 \\
.37 \\
.80 \\
1.61 \\
.62\end{array}$ \\
\hline $\begin{array}{l}51 \\
52 \\
53 \\
54 \\
55\end{array}$ & $\begin{array}{l}27.6 \\
56.0 \\
59.8 \\
43.8 \\
52.4\end{array}$ & $\begin{array}{l}283.4 \\
366.4 \\
356.8 \\
325.9 \\
329.4\end{array}$ & $\begin{array}{l}7.82 \\
4.93 \\
5.63 \\
5.83 \\
6.97\end{array}$ & $\begin{array}{l}11.30 \\
14.04 \\
15.00 \\
15.27 \\
19.80\end{array}$ & $\begin{array}{r}.27 \\
-3.38 \\
-4.72 \\
-3.24 \\
-4.15\end{array}$ & $\begin{array}{l}54.9 \\
65.3 \\
51.0 \\
40.5 \\
45.3\end{array}$ & $\begin{array}{l}85.4 \\
92.8 \\
91.3 \\
64.6 \\
77.8\end{array}$ & $\begin{array}{l}43.4 \\
32.3 \\
21.8 \\
19.3 \\
15.1\end{array}$ & $\begin{array}{r}2.65 \\
.94 \\
1.99 \\
1.02 \\
.81\end{array}$ & $\begin{array}{l}5.21 \\
2.01 \\
4.80 \\
1.64 \\
1.23\end{array}$ & $\begin{array}{l}.26 \\
.29 \\
.35 \\
.29 \\
.33\end{array}$ \\
\hline $\begin{array}{l}56 \\
57 \\
58 \\
59 \\
60\end{array}$ & $\begin{array}{l}52.4 \\
34.7 \\
47.5 \\
38.4 \\
44.1\end{array}$ & $\begin{array}{l}328.8 \\
293.8 \\
315.0 \\
317.5 \\
311.4\end{array}$ & $\begin{array}{r}9.85 \\
14.87 \\
12.82 \\
14.12 \\
14.80\end{array}$ & $\begin{array}{l}22.79 \\
22.25 \\
21.92 \\
21.75 \\
24.05\end{array}$ & $\begin{array}{r}-2.29 \\
6.86 \\
.44 \\
3.58 \\
4.45\end{array}$ & $\begin{array}{l}40.0 \\
30.3 \\
44.2 \\
30.8 \\
34.2\end{array}$ & $\begin{array}{l}73.3 \\
43.4 \\
82.2 \\
60.0 \\
64.3\end{array}$ & $\begin{array}{l}13.0 \\
21.9 \\
24.3 \\
14.6 \\
16.6\end{array}$ & $\begin{array}{l}1.07 \\
1.97 \\
1.35 \\
1.97 \\
1.84\end{array}$ & $\begin{array}{l}2.70 \\
4.63 \\
2.72 \\
3.06 \\
3.58\end{array}$ & $\begin{array}{l}.50 \\
.52 \\
.32 \\
.59 \\
.36\end{array}$ \\
\hline $\begin{array}{l}61 \\
62 \\
63 \\
64 \\
65\end{array}$ & $\begin{array}{l}54.1 \\
54.8 \\
52.8 \\
30.2 \\
30.8\end{array}$ & $\begin{array}{l}318.5 \\
321.0 \\
312.9 \\
275.6 \\
329.7\end{array}$ & $\begin{array}{l}12.89 \\
13.50 \\
14.07 \\
14.80 \\
15.88\end{array}$ & $\begin{array}{l}26.30 \\
26.85 \\
26.65 \\
22.73 \\
23.57\end{array}$ & $\begin{array}{r}.73 \\
2.48 \\
2.09 \\
3.55 \\
8.90\end{array}$ & $\begin{array}{l}44.1 \\
40.7 \\
38.5 \\
38.7 \\
44.3\end{array}$ & $\begin{array}{l}78.5 \\
67.4 \\
69.9 \\
67.5 \\
64.1\end{array}$ & $\begin{array}{l}15.7 \\
14.2 \\
14.0 \\
22.1 \\
25.0\end{array}$ & $\begin{array}{r}.84 \\
.95 \\
.96 \\
1.64 \\
1.63\end{array}$ & $\begin{array}{l}1.82 \\
1.67 \\
1.81 \\
3.38 \\
4.94\end{array}$ & $\begin{array}{l}.42 \\
.27 \\
.31 \\
.35 \\
.46\end{array}$ \\
\hline $\begin{array}{l}66 \\
67 \\
68 \\
69 \\
70\end{array}$ & $\begin{array}{l}42.7 \\
51.5 \\
51.2 \\
42.7 \\
12.6\end{array}$ & $\begin{array}{l}348.8 \\
320.9 \\
305.2 \\
289.8 \\
200.3\end{array}$ & $\begin{array}{l}16.00 \\
13.33 \\
12.39 \\
16.52 \\
14.21\end{array}$ & $\begin{array}{l}24.33 \\
24.81 \\
24.61 \\
25.32 \\
19.43\end{array}$ & $\begin{array}{r}8.58 \\
2.31 \\
.81 \\
5.14 \\
7.55\end{array}$ & $\begin{array}{l}42.8 \\
38.1 \\
34.3 \\
27.3 \\
36.0\end{array}$ & $\begin{array}{l}62.3 \\
77.8 \\
67.5 \\
49.2 \\
53.5\end{array}$ & \begin{tabular}{r|}
19.6 \\
9.8 \\
8.7 \\
11.4 \\
23.2
\end{tabular} & $\begin{array}{r}1.75 \\
1.26 \\
.93 \\
2.19 \\
2.30\end{array}$ & $\begin{array}{l}2.84 \\
2.51 \\
1.57 \\
4.65 \\
3.98\end{array}$ & $\begin{array}{l}.35 \\
.58 \\
.30 \\
.47 \\
.59\end{array}$ \\
\hline
\end{tabular}


Table 3. Daily mean, maximum, and minimum micrometeorological data measured by micromet station at Rogers Spring Site 1, 1994-Continued

\begin{tabular}{|c|c|c|c|c|c|c|c|c|c|c|c|}
\hline \multirow[t]{2}{*}{$\begin{array}{l}\text { Calendar } \\
\text { day }\end{array}$} & \multicolumn{2}{|c|}{$\begin{array}{l}\text { Net radiation, } \\
\text { in watts per } \\
\text { square meter }\end{array}$} & \multicolumn{3}{|c|}{$\begin{array}{l}\text { Air temperature at } 0.7 \text { meter, } \\
\text { in degrees Celsius }\end{array}$} & \multicolumn{3}{|c|}{$\begin{array}{l}\text { Relative humidity at } 0.7 \text { meter, } \\
\text { in percent }\end{array}$} & \multicolumn{3}{|c|}{$\begin{array}{l}\text { Windspeed at } 0.7 \text { meter, } \\
\text { in meters per second }\end{array}$} \\
\hline & Mean & Maximum & Mean & Maximum & Minimum & Mean & Maximum & Minimum & Mean & Maximum & Minimum \\
\hline $\begin{array}{l}71 \\
72 \\
73 \\
74 \\
75\end{array}$ & $\begin{array}{l}53.3 \\
50.4 \\
60.0 \\
53.3 \\
44.6\end{array}$ & $\begin{array}{l}337.4 \\
329.5 \\
325.1 \\
311.0 \\
334.2\end{array}$ & $\begin{array}{l}16.79 \\
17.02 \\
15.46 \\
18.37 \\
22.82\end{array}$ & $\begin{array}{l}24.32 \\
27.30 \\
29.39 \\
30.43 \\
28.06\end{array}$ & $\begin{array}{r}9.44 \\
5.87 \\
1.13 \\
4.12 \\
18.55\end{array}$ & $\begin{array}{l}25.8 \\
20.7 \\
28.0 \\
23.6 \\
12.5\end{array}$ & $\begin{array}{l}39.7 \\
38.8 \\
60.4 \\
53.1 \\
19.2\end{array}$ & $\begin{array}{r}13.9 \\
6.0 \\
8.7 \\
7.7 \\
7.2\end{array}$ & $\begin{array}{r}3.07 \\
1.86 \\
.95 \\
1.65 \\
4.01\end{array}$ & $\begin{array}{l}5.01 \\
3.24 \\
1.54 \\
3.91 \\
5.63\end{array}$ & $\begin{array}{r}1.62 \\
.44 \\
.42 \\
.35 \\
1.98\end{array}$ \\
\hline $\begin{array}{l}76 \\
77 \\
78 \\
79 \\
80\end{array}$ & $\begin{array}{l}56.9 \\
64.9 \\
41.8 \\
74.5 \\
68.1\end{array}$ & $\begin{array}{l}320.2 \\
356.4 \\
337.3 \\
354.1 \\
361.3\end{array}$ & $\begin{array}{l}19.70 \\
20.07 \\
16.18 \\
13.68 \\
15.76\end{array}$ & $\begin{array}{l}26.29 \\
25.02 \\
20.56 \\
22.12 \\
23.75\end{array}$ & $\begin{array}{r}10.88 \\
15.33 \\
10.33 \\
6.99 \\
3.59\end{array}$ & $\begin{array}{l}19.7 \\
27.9 \\
50.4 \\
54.0 \\
43.9\end{array}$ & $\begin{array}{l}47.7 \\
50.2 \\
74.1 \\
91.1 \\
70.5\end{array}$ & $\begin{array}{r}9.1 \\
15.9 \\
39.6 \\
22.8 \\
28.0\end{array}$ & $\begin{array}{l}1.47 \\
4.19 \\
3.79 \\
1.04 \\
2.45\end{array}$ & $\begin{array}{l}3.50 \\
6.56 \\
6.43 \\
2.02 \\
4.56\end{array}$ & $\begin{array}{r}.24 \\
1.02 \\
.70 \\
.24 \\
.46\end{array}$ \\
\hline $\begin{array}{l}81 \\
82 \\
83 \\
84 \\
85\end{array}$ & $\begin{array}{l}58.7 \\
66.6 \\
50.9 \\
99.2 \\
92.2\end{array}$ & $\begin{array}{l}363.9 \\
352.5 \\
309.0 \\
528.2 \\
463.8\end{array}$ & $\begin{array}{l}14.03 \\
10.79 \\
13.40 \\
10.34 \\
12.89\end{array}$ & $\begin{array}{l}18.62 \\
18.01 \\
19.25 \\
16.40 \\
22.12\end{array}$ & $\begin{array}{r}2.86 \\
.11 \\
8.85 \\
5.86 \\
1.39\end{array}$ & $\begin{array}{l}29.7 \\
22.8 \\
36.3 \\
57.6 \\
44.9\end{array}$ & $\begin{array}{l}49.1 \\
51.6 \\
51.9 \\
90.7 \\
85.7\end{array}$ & $\begin{array}{l}12.1 \\
10.0 \\
19.0 \\
25.0 \\
17.4\end{array}$ & $\begin{array}{l}4.51 \\
1.57 \\
1.96 \\
2.18 \\
1.59\end{array}$ & $\begin{array}{l}8.53 \\
2.57 \\
4.76 \\
4.12 \\
3.35\end{array}$ & $\begin{array}{l}.66 \\
.45 \\
.64 \\
.63 \\
.45\end{array}$ \\
\hline $\begin{array}{l}86 \\
87 \\
88 \\
89 \\
90\end{array}$ & $\begin{array}{l}78.0 \\
71.8 \\
80.3 \\
80.0 \\
84.6\end{array}$ & $\begin{array}{l}406.7 \\
397.2 \\
377.2 \\
388.2 \\
407.5\end{array}$ & $\begin{array}{l}15.57 \\
16.26 \\
16.66 \\
18.77 \\
18.12\end{array}$ & $\begin{array}{l}25.73 \\
26.46 \\
27.47 \\
28.56 \\
28.99\end{array}$ & $\begin{array}{l}5.23 \\
1.87 \\
4.41 \\
8.45 \\
4.84\end{array}$ & $\begin{array}{l}24.8 \\
27.0 \\
28.4 \\
25.9 \\
27.5\end{array}$ & $\begin{array}{l}52.5 \\
62.2 \\
59.3 \\
51.7 \\
60.4\end{array}$ & $\begin{array}{r}7.3 \\
11.3 \\
10.1 \\
9.9 \\
10.5\end{array}$ & $\begin{array}{l}1.69 \\
1.80 \\
1.04 \\
1.18 \\
1.85\end{array}$ & $\begin{array}{l}3.32 \\
4.03 \\
1.99 \\
2.07 \\
4.36\end{array}$ & $\begin{array}{l}.57 \\
.47 \\
.35 \\
.44 \\
.32\end{array}$ \\
\hline $\begin{array}{l}91 \\
92 \\
93 \\
94 \\
95\end{array}$ & $\begin{array}{l}84.4 \\
73.3 \\
44.2 \\
77.3 \\
79.5\end{array}$ & $\begin{array}{l}403.9 \\
373.4 \\
337.5 \\
402.6 \\
386.9\end{array}$ & $\begin{array}{l}16.72 \\
18.56 \\
20.32 \\
16.22 \\
14.47\end{array}$ & $\begin{array}{l}26.94 \\
27.38 \\
25.79 \\
24.20 \\
23.02\end{array}$ & $\begin{array}{r}1.99 \\
6.21 \\
12.99 \\
5.85 \\
4.87\end{array}$ & $\begin{array}{l}29.7 \\
24.1 \\
26.3 \\
26.7 \\
21.9\end{array}$ & $\begin{array}{l}75.3 \\
56.0 \\
47.6 \\
64.1 \\
35.8\end{array}$ & $\begin{array}{r}9.7 \\
10.1 \\
16.2 \\
5.9 \\
9.2\end{array}$ & $\begin{array}{l}1.96 \\
1.17 \\
3.20 \\
2.56 \\
1.25\end{array}$ & $\begin{array}{l}4.85 \\
2.44 \\
5.75 \\
4.84 \\
2.44\end{array}$ & $\begin{array}{l}.28 \\
.32 \\
.84 \\
.63 \\
.39\end{array}$ \\
\hline $\begin{array}{r}96 \\
97 \\
98 \\
99 \\
100\end{array}$ & $\begin{array}{l}53.1 \\
83.9 \\
59.7 \\
62.7 \\
78.7\end{array}$ & $\begin{array}{l}390.9 \\
396.1 \\
382.3 \\
398.2 \\
422.7\end{array}$ & $\begin{array}{l}16.11 \\
16.38 \\
16.21 \\
12.95 \\
15.18\end{array}$ & $\begin{array}{l}24.22 \\
24.11 \\
21.51 \\
19.34 \\
22.66\end{array}$ & $\begin{array}{r}4.96 \\
4.05 \\
10.91 \\
6.96 \\
7.29\end{array}$ & $\begin{array}{l}25.4 \\
30.9 \\
33.5 \\
42.6 \\
30.7\end{array}$ & $\begin{array}{l}49.1 \\
68.6 \\
49.2 \\
60.2 \\
53.1\end{array}$ & $\begin{array}{l}13.9 \\
12.8 \\
22.0 \\
26.6 \\
13.1\end{array}$ & $\begin{array}{l}1.72 \\
1.25 \\
2.48 \\
1.57 \\
3.17\end{array}$ & $\begin{array}{l}3.42 \\
1.80 \\
4.21 \\
2.92 \\
4.90\end{array}$ & $\begin{array}{r}.44 \\
.43 \\
.95 \\
.56 \\
1.66\end{array}$ \\
\hline $\begin{array}{l}101 \\
102 \\
103 \\
104 \\
105\end{array}$ & $\begin{array}{l}88.9 \\
83.0 \\
88.4 \\
74.2 \\
84.6\end{array}$ & $\begin{array}{l}401.0 \\
368.1 \\
400.3 \\
356.4 \\
391.2\end{array}$ & $\begin{array}{l}15.81 \\
17.13 \\
18.92 \\
20.14 \\
22.29\end{array}$ & $\begin{array}{l}25.58 \\
27.94 \\
29.80 \\
30.73 \\
31.10\end{array}$ & $\begin{array}{r}4.45 \\
5.30 \\
4.30 \\
6.57 \\
11.42\end{array}$ & $\begin{array}{l}35.1 \\
30.8 \\
24.1 \\
29.5 \\
22.1\end{array}$ & $\begin{array}{l}61.8 \\
58.5 \\
58.0 \\
69.5 \\
51.8\end{array}$ & $\begin{array}{r}17.1 \\
12.5 \\
9.3 \\
10.3 \\
10.3\end{array}$ & $\begin{array}{l}1.32 \\
1.03 \\
1.36 \\
1.48 \\
1.74\end{array}$ & $\begin{array}{l}2.87 \\
2.06 \\
2.50 \\
3.61 \\
3.65\end{array}$ & $\begin{array}{l}.36 \\
.39 \\
.36 \\
.27 \\
.35\end{array}$ \\
\hline
\end{tabular}


Table 3. Daily mean, maximum, and minimum micrometeorological data measured by micromet station at Rogers Spring Site 1 , 1994-Continued

\begin{tabular}{|c|c|c|c|c|c|c|c|c|c|c|c|}
\hline \multirow{2}{*}{$\begin{array}{l}\text { Calendar } \\
\text { day }\end{array}$} & \multicolumn{2}{|c|}{$\begin{array}{l}\text { Net radiation, } \\
\text { in watts per } \\
\text { square meter }\end{array}$} & \multicolumn{3}{|c|}{$\begin{array}{l}\text { Air temperature at } 0.7 \text { meter, } \\
\text { in degrees Celsius }\end{array}$} & \multicolumn{3}{|c|}{$\begin{array}{l}\text { Relative humidity at } 0.7 \text { meter, } \\
\text { in percent }\end{array}$} & \multicolumn{3}{|c|}{$\begin{array}{l}\text { Windspeed at } 0.7 \text { meter, } \\
\text { in meters per second }\end{array}$} \\
\hline & Mean & Maximum & Mean & Maximum & Minimum & Mean & Maximum & Minimum & Mean & Maximum & Minimum \\
\hline $\begin{array}{l}106 \\
107 \\
108 \\
109 \\
110\end{array}$ & $\begin{array}{l}75.0 \\
75.2 \\
88.5 \\
96.4 \\
99.7\end{array}$ & $\begin{array}{l}346.4 \\
362.0 \\
403.8 \\
398.0 \\
407.0\end{array}$ & $\begin{array}{l}21.79 \\
23.65 \\
22.62 \\
22.70 \\
23.83\end{array}$ & $\begin{array}{l}33.32 \\
34.25 \\
34.42 \\
35.10 \\
35.75\end{array}$ & $\begin{array}{r}8.77 \\
13.29 \\
11.76 \\
9.73 \\
10.89\end{array}$ & $\begin{array}{l}25.7 \\
20.5 \\
25.3 \\
27.4 \\
24.8\end{array}$ & $\begin{array}{l}46.8 \\
38.7 \\
45.9 \\
50.5 \\
52.6\end{array}$ & $\begin{array}{r}8.6 \\
8.4 \\
9.1 \\
10.7 \\
8.1\end{array}$ & $\begin{array}{l}1.06 \\
1.55 \\
1.12 \\
1.09 \\
1.50\end{array}$ & $\begin{array}{l}2.28 \\
3.86 \\
2.56 \\
2.40 \\
3.90\end{array}$ & $\begin{array}{r}0.26 \\
.46 \\
.47 \\
.21 \\
.42\end{array}$ \\
\hline $\begin{array}{l}111 \\
112 \\
113 \\
114 \\
115\end{array}$ & $\begin{array}{l}90.9 \\
86.7 \\
75.0 \\
72.2 \\
73.1\end{array}$ & $\begin{array}{l}422.9 \\
406.1 \\
377.2 \\
381.4 \\
415.0\end{array}$ & $\begin{array}{l}25.27 \\
21.72 \\
18.62 \\
11.63 \\
12.75\end{array}$ & $\begin{array}{l}32.26 \\
27.91 \\
24.16 \\
17.14 \\
20.00\end{array}$ & $\begin{array}{r}17.91 \\
13.52 \\
12.04 \\
5.01 \\
6.39\end{array}$ & $\begin{array}{l}18.2 \\
20.9 \\
25.2 \\
43.2 \\
40.4\end{array}$ & $\begin{array}{l}28.2 \\
40.2 \\
44.2 \\
75.2 \\
59.1\end{array}$ & $\begin{array}{r}7.1 \\
11.7 \\
11.4 \\
24.8 \\
19.2\end{array}$ & $\begin{array}{l}3.34 \\
3.06 \\
5.88 \\
2.93 \\
3.35\end{array}$ & $\begin{array}{l}5.90 \\
5.35 \\
7.68 \\
5.81 \\
5.46\end{array}$ & $\begin{array}{r}.90 \\
.84 \\
4.13 \\
.21 \\
1.17\end{array}$ \\
\hline $\begin{array}{l}116 \\
117 \\
118 \\
119 \\
120\end{array}$ & $\begin{array}{r}72.9 \\
92.3 \\
109.9 \\
132.3 \\
82.4\end{array}$ & $\begin{array}{l}428.9 \\
512.3 \\
535.0 \\
503.3 \\
408.2\end{array}$ & $\begin{array}{l}10.63 \\
11.60 \\
12.86 \\
14.64 \\
17.99\end{array}$ & $\begin{array}{l}19.21 \\
17.67 \\
18.84 \\
24.30 \\
24.85\end{array}$ & $\begin{array}{r}3.72 \\
6.93 \\
7.08 \\
3.04 \\
10.32\end{array}$ & $\begin{array}{l}57.0 \\
74.3 \\
64.7 \\
49.0 \\
39.3\end{array}$ & $\begin{array}{l}90.5 \\
96.4 \\
95.3 \\
93.3 \\
72.8\end{array}$ & $\begin{array}{l}22.8 \\
43.7 \\
33.2 \\
16.4 \\
22.8\end{array}$ & $\begin{array}{l}1.34 \\
1.23 \\
1.16 \\
1.04 \\
1.71\end{array}$ & $\begin{array}{l}3.47 \\
2.43 \\
3.46 \\
2.43 \\
3.95\end{array}$ & $\begin{array}{l}.21 \\
.36 \\
.21 \\
.28 \\
.38\end{array}$ \\
\hline $\begin{array}{l}121 \\
122 \\
123 \\
124 \\
125\end{array}$ & $\begin{array}{r}105.5 \\
101.4 \\
91.9 \\
112.5 \\
92.1\end{array}$ & $\begin{array}{l}434.1 \\
395.5 \\
417.4 \\
438.6 \\
415.5\end{array}$ & $\begin{array}{l}17.85 \\
19.70 \\
20.55 \\
23.72 \\
24.67\end{array}$ & $\begin{array}{l}27.68 \\
29.38 \\
29.83 \\
32.20 \\
30.97\end{array}$ & $\begin{array}{r}8.46 \\
7.52 \\
9.09 \\
11.27 \\
18.86\end{array}$ & $\begin{array}{l}41.5 \\
33.5 \\
29.7 \\
27.4 \\
20.8\end{array}$ & $\begin{array}{l}71.9 \\
73.5 \\
63.1 \\
49.5 \\
33.7\end{array}$ & $\begin{array}{l}16.7 \\
13.3 \\
12.9 \\
14.6 \\
11.4\end{array}$ & $\begin{array}{l}1.10 \\
1.39 \\
1.29 \\
2.42 \\
3.32\end{array}$ & $\begin{array}{l}2.46 \\
3.30 \\
2.14 \\
5.09 \\
4.50\end{array}$ & $\begin{array}{l}.34 \\
.29 \\
.41 \\
.52 \\
.87\end{array}$ \\
\hline $\begin{array}{l}126 \\
127 \\
128 \\
129 \\
130\end{array}$ & $\begin{array}{r}114.3 \\
88.9 \\
72.1 \\
72.0 \\
107.1\end{array}$ & $\begin{array}{l}446.1 \\
419.9 \\
353.5 \\
341.6 \\
434.8\end{array}$ & $\begin{array}{l}18.23 \\
15.96 \\
17.05 \\
19.85 \\
22.47\end{array}$ & $\begin{array}{l}24.04 \\
21.62 \\
22.58 \\
29.53 \\
31.88\end{array}$ & $\begin{array}{r}13.04 \\
9.20 \\
11.18 \\
8.64 \\
8.85\end{array}$ & $\begin{array}{l}34.0 \\
42.6 \\
47.1 \\
41.4 \\
37.1\end{array}$ & $\begin{array}{l}53.5 \\
65.4 \\
74.2 \\
77.6 \\
72.6\end{array}$ & $\begin{array}{l}19.0 \\
25.4 \\
27.8 \\
17.5 \\
18.1\end{array}$ & $\begin{array}{l}3.53 \\
2.15 \\
2.02 \\
1.53 \\
1.22\end{array}$ & $\begin{array}{l}5.49 \\
3.30 \\
3.22 \\
3.83 \\
2.00\end{array}$ & $\begin{array}{r}1.58 \\
.91 \\
.22 \\
.63 \\
.35\end{array}$ \\
\hline $\begin{array}{l}131 \\
132 \\
133 \\
134 \\
135\end{array}$ & $\begin{array}{l}106.3 \\
116.4 \\
119.8 \\
112.4 \\
102.3\end{array}$ & $\begin{array}{l}429.2 \\
426.1 \\
431.2 \\
431.5 \\
462.9\end{array}$ & $\begin{array}{l}26.60 \\
25.67 \\
24.80 \\
26.27 \\
26.76\end{array}$ & $\begin{array}{l}35.12 \\
34.09 \\
34.41 \\
35.51 \\
32.39\end{array}$ & $\begin{array}{l}15.53 \\
12.66 \\
12.54 \\
10.76 \\
21.37\end{array}$ & $\begin{array}{l}32.6 \\
35.9 \\
41.9 \\
28.7 \\
16.5\end{array}$ & $\begin{array}{l}62.1 \\
66.3 \\
86.0 \\
70.7 \\
26.0\end{array}$ & $\begin{array}{r}16.1 \\
20.5 \\
18.4 \\
12.3 \\
6.5\end{array}$ & $\begin{array}{l}1.58 \\
2.03 \\
1.07 \\
2.25 \\
5.62\end{array}$ & $\begin{array}{l}3.48 \\
3.89 \\
2.13 \\
4.83 \\
7.51\end{array}$ & $\begin{array}{r}.64 \\
.43 \\
.39 \\
.30 \\
2.44\end{array}$ \\
\hline $\begin{array}{l}136 \\
137 \\
138 \\
139 \\
140\end{array}$ & $\begin{array}{l}115.7 \\
105.2 \\
119.0 \\
109.2 \\
111.9\end{array}$ & $\begin{array}{l}466.7 \\
461.5 \\
460.0 \\
421.5 \\
423.6\end{array}$ & $\begin{array}{l}19.40 \\
16.22 \\
15.41 \\
15.29 \\
16.06\end{array}$ & $\begin{array}{l}25.27 \\
22.24 \\
21.75 \\
23.62 \\
26.75\end{array}$ & $\begin{array}{l}9.57 \\
9.98 \\
9.00 \\
7.79 \\
3.52\end{array}$ & $\begin{array}{l}32.4 \\
33.1 \\
38.6 \\
43.5 \\
37.6\end{array}$ & $\begin{array}{l}59.9 \\
50.9 \\
58.2 \\
70.0 \\
74.3\end{array}$ & $\begin{array}{l}13.1 \\
21.0 \\
20.4 \\
19.5 \\
11.0\end{array}$ & $\begin{array}{l}4.79 \\
3.41 \\
3.08 \\
1.48 \\
0.96\end{array}$ & $\begin{array}{l}7.04 \\
4.69 \\
4.61 \\
2.64 \\
2.07\end{array}$ & $\begin{array}{r}.63 \\
1.35 \\
1.15 \\
.31 \\
.20\end{array}$ \\
\hline
\end{tabular}


Table 3. Daily mean, maximum, and minimum micrometeorological data measured by micromet station at Rogers Spring Site 1, 1994-Continued

\begin{tabular}{|c|c|c|c|c|c|c|c|c|c|c|c|}
\hline $\begin{array}{c}\text { Calendar } \\
\text { day }\end{array}$ & \multicolumn{2}{|c|}{$\begin{array}{l}\text { Net radiation, } \\
\text { in watts per } \\
\text { square meter }\end{array}$} & \multicolumn{3}{|c|}{$\begin{array}{l}\text { Air temperature at } 0.7 \text { meter, } \\
\text { in degrees Celsius }\end{array}$} & \multicolumn{3}{|c|}{$\begin{array}{l}\text { Relative humidity at } 0.7 \text { meter, } \\
\text { in percent }\end{array}$} & \multicolumn{3}{|c|}{$\begin{array}{l}\text { Windspeed at } 0.7 \text { meter, } \\
\text { in meters per second }\end{array}$} \\
\hline & Mean & Maximum & Mean & Maximum & Minimum & Mean & Maximum & Minimum & Mean & Maximum & Minimum \\
\hline $\begin{array}{l}141 \\
142 \\
143 \\
161 \\
162\end{array}$ & $\begin{array}{l}111.5 \\
112.6 \\
116.3 \\
125.8 \\
125.8\end{array}$ & $\begin{array}{l}422.1 \\
424.1 \\
429.3 \\
427.4 \\
438.0\end{array}$ & $\begin{array}{l}18.73 \\
20.96 \\
22.72 \\
27.88 \\
29.98\end{array}$ & $\begin{array}{l}30.56 \\
32.56 \\
34.39 \\
41.05 \\
40.74\end{array}$ & $\begin{array}{r}3.80 \\
6.97 \\
9.22 \\
11.30 \\
14.65\end{array}$ & $\begin{array}{l}23.1 \\
21.7 \\
29.0 \\
19.6 \\
19.5\end{array}$ & $\begin{array}{l}51.0 \\
44.3 \\
57.3 \\
43.3 \\
52.8\end{array}$ & $\begin{array}{l}6.5 \\
7.5 \\
9.2 \\
5.0 \\
7.8\end{array}$ & $\begin{array}{l}1.35 \\
1.46 \\
1.11 \\
1.12 \\
1.62\end{array}$ & $\begin{array}{l}3.18 \\
3.24 \\
2.43 \\
2.27 \\
3.28\end{array}$ & $\begin{array}{r}0.37 \\
.46 \\
.40 \\
.38 \\
.41\end{array}$ \\
\hline $\begin{array}{l}163 \\
164 \\
165 \\
166 \\
167\end{array}$ & $\begin{array}{l}123.7 \\
112.7 \\
125.1 \\
123.2 \\
123.3\end{array}$ & $\begin{array}{l}452.9 \\
468.6 \\
455.4 \\
465.9 \\
456.2\end{array}$ & $\begin{array}{l}31.68 \\
31.27 \\
29.75 \\
26.61 \\
24.35\end{array}$ & $\begin{array}{l}39.24 \\
37.61 \\
36.74 \\
33.68 \\
32.93\end{array}$ & $\begin{array}{l}23.14 \\
23.01 \\
23.49 \\
19.59 \\
12.37\end{array}$ & $\begin{array}{l}16.9 \\
16.2 \\
22.0 \\
19.1 \\
19.4\end{array}$ & $\begin{array}{l}36.3 \\
33.1 \\
35.3 \\
36.0 \\
45.1\end{array}$ & $\begin{array}{r}9.0 \\
5.7 \\
13.4 \\
7.7 \\
6.4\end{array}$ & $\begin{array}{l}2.05 \\
3.15 \\
3.73 \\
3.90 \\
2.22\end{array}$ & $\begin{array}{l}3.81 \\
4.87 \\
5.02 \\
5.73 \\
4.19\end{array}$ & $\begin{array}{r}.49 \\
.58 \\
2.20 \\
1.29 \\
.44\end{array}$ \\
\hline $\begin{array}{l}168 \\
169 \\
170 \\
171 \\
172\end{array}$ & $\begin{array}{l}127.1 \\
130.2 \\
130.7 \\
130.7 \\
127.3\end{array}$ & $\begin{array}{l}465.1 \\
464.8 \\
448.0 \\
457.5 \\
449.1\end{array}$ & $\begin{array}{l}27.95 \\
27.98 \\
27.55 \\
28.67 \\
31.47\end{array}$ & $\begin{array}{l}35.99 \\
36.90 \\
38.24 \\
38.70 \\
39.14\end{array}$ & $\begin{array}{l}15.49 \\
17.66 \\
11.17 \\
10.92 \\
21.88\end{array}$ & $\begin{array}{l}14.0 \\
16.3 \\
18.3 \\
19.8 \\
20.7\end{array}$ & $\begin{array}{l}37.1 \\
31.0 \\
53.7 \\
56.4 \\
45.1\end{array}$ & $\begin{array}{l}4.5 \\
6.4 \\
5.3 \\
6.6 \\
7.4\end{array}$ & $\begin{array}{l}2.73 \\
2.08 \\
1.61 \\
1.97 \\
1.87\end{array}$ & $\begin{array}{l}4.97 \\
4.34 \\
3.90 \\
3.60 \\
3.00\end{array}$ & $\begin{array}{l}.92 \\
.45 \\
.42 \\
.40 \\
.81\end{array}$ \\
\hline $\begin{array}{l}173 \\
174 \\
175 \\
176 \\
177\end{array}$ & $\begin{array}{l}128.0 \\
124.5 \\
128.5 \\
125.9 \\
121.8\end{array}$ & $\begin{array}{l}458.7 \\
454.5 \\
435.6 \\
458.2 \\
451.7\end{array}$ & $\begin{array}{l}31.96 \\
31.84 \\
30.25 \\
33.87 \\
36.16\end{array}$ & $\begin{array}{l}39.75 \\
41.12 \\
42.01 \\
42.33 \\
43.69\end{array}$ & $\begin{array}{l}20.00 \\
18.74 \\
11.68 \\
17.49 \\
29.40\end{array}$ & $\begin{array}{r}14.0 \\
11.6 \\
19.4 \\
11.4 \\
9.3\end{array}$ & $\begin{array}{l}32.9 \\
39.5 \\
62.2 \\
35.9 \\
15.1\end{array}$ & $\begin{array}{l}8.5 \\
3.5 \\
5.5 \\
4.5 \\
5.4\end{array}$ & $\begin{array}{l}2.15 \\
2.04 \\
1.37 \\
2.91 \\
3.39\end{array}$ & $\begin{array}{l}3.94 \\
3.69 \\
2.91 \\
4.54 \\
5.59\end{array}$ & $\begin{array}{r}.66 \\
.42 \\
.28 \\
.67 \\
1.70\end{array}$ \\
\hline $\begin{array}{l}178 \\
188 \\
189 \\
190 \\
191\end{array}$ & $\begin{array}{l}113.0 \\
129.3 \\
130.6 \\
133.0 \\
133.6\end{array}$ & $\begin{array}{l}438.4 \\
446.5 \\
437.9 \\
459.1 \\
466.2\end{array}$ & $\begin{array}{l}34.65 \\
30.16 \\
28.67 \\
31.55 \\
33.13\end{array}$ & $\begin{array}{l}43.32 \\
39.61 \\
41.91 \\
41.78 \\
40.54\end{array}$ & $\begin{array}{l}24.13 \\
18.47 \\
13.65 \\
14.76 \\
22.40\end{array}$ & $\begin{array}{l}18.0 \\
17.2 \\
21.7 \\
15.9 \\
13.5\end{array}$ & $\begin{array}{l}36.5 \\
57.3 \\
45.7 \\
52.2 \\
26.3\end{array}$ & $\begin{array}{l}8.8 \\
5.5 \\
4.5 \\
4.8 \\
6.4\end{array}$ & $\begin{array}{r}1.56 \\
1.61 \\
.84 \\
1.81 \\
2.56\end{array}$ & $\begin{array}{l}3.77 \\
4.63 \\
1.62 \\
3.61 \\
4.31\end{array}$ & $\begin{array}{l}.41 \\
.24 \\
.32 \\
.42 \\
.64\end{array}$ \\
\hline $\begin{array}{l}192 \\
193 \\
194 \\
195 \\
196\end{array}$ & $\begin{array}{l}132.5 \\
130.4 \\
132.0 \\
127.6 \\
135.8\end{array}$ & $\begin{array}{l}443.3 \\
436.1 \\
445.7 \\
467.9 \\
457.7\end{array}$ & $\begin{array}{l}30.35 \\
30.65 \\
31.16 \\
31.81 \\
31.85\end{array}$ & $\begin{array}{l}40.32 \\
42.07 \\
41.47 \\
41.44 \\
40.60\end{array}$ & $\begin{array}{l}15.49 \\
14.10 \\
19.61 \\
17.05 \\
19.78\end{array}$ & $\begin{array}{l}19.3 \\
21.9 \\
16.9 \\
17.2 \\
17.6\end{array}$ & $\begin{array}{l}48.6 \\
59.0 \\
36.6 \\
51.1 \\
35.9\end{array}$ & $\begin{array}{l}6.8 \\
6.7 \\
4.1 \\
5.7 \\
7.6\end{array}$ & $\begin{array}{l}1.37 \\
1.29 \\
1.66 \\
1.86 \\
1.97\end{array}$ & $\begin{array}{l}2.56 \\
2.59 \\
3.67 \\
4.22 \\
3.71\end{array}$ & $\begin{array}{l}.47 \\
.41 \\
.38 \\
.34 \\
.48\end{array}$ \\
\hline $\begin{array}{l}197 \\
198 \\
199 \\
200 \\
201\end{array}$ & $\begin{array}{l}124.2 \\
113.3 \\
126.3 \\
125.6 \\
135.5\end{array}$ & $\begin{array}{l}461.1 \\
454.8 \\
429.4 \\
430.8 \\
460.9\end{array}$ & $\begin{array}{l}31.80 \\
32.80 \\
32.93 \\
30.39 \\
29.52\end{array}$ & $\begin{array}{l}40.35 \\
40.84 \\
38.88 \\
38.03 \\
38.39\end{array}$ & $\begin{array}{l}18.28 \\
24.08 \\
25.18 \\
21.88 \\
19.65\end{array}$ & $\begin{array}{l}14.8 \\
21.5 \\
24.0 \\
36.3 \\
43.0\end{array}$ & $\begin{array}{l}37.0 \\
44.9 \\
38.2 \\
70.3 \\
83.3\end{array}$ & $\begin{array}{r}3.4 \\
10.5 \\
15.1 \\
17.8 \\
18.5\end{array}$ & $\begin{array}{l}1.98 \\
1.34 \\
2.96 \\
1.95 \\
1.57\end{array}$ & $\begin{array}{l}3.46 \\
2.61 \\
7.77 \\
4.97 \\
2.97\end{array}$ & $\begin{array}{l}.49 \\
.35 \\
.53 \\
.25 \\
.67\end{array}$ \\
\hline
\end{tabular}


Table 3. Daily mean, maximum, and minimum micrometeorological data measured by micromet station at Rogers Spring Site 1, 1994-Continued

\begin{tabular}{|c|c|c|c|c|c|c|c|c|c|c|c|}
\hline \multirow{2}{*}{$\begin{array}{l}\text { Calendar } \\
\text { day }\end{array}$} & \multicolumn{2}{|c|}{$\begin{array}{l}\text { Net radiation, } \\
\text { in watts per } \\
\text { square meter }\end{array}$} & \multicolumn{3}{|c|}{$\begin{array}{l}\text { Air temperature at } 0.7 \text { meter, } \\
\text { in degrees Celsius }\end{array}$} & \multicolumn{3}{|c|}{$\begin{array}{l}\text { Relative humidity at } 0.7 \text { meter, } \\
\text { in percent }\end{array}$} & \multicolumn{3}{|c|}{$\begin{array}{l}\text { Windspeed at } 0.7 \text { meter, } \\
\text { in meters per second }\end{array}$} \\
\hline & Mean & Maximum & Mean & Maximum & Minimum & Mean & Maximum & Minimum & Mean & Maximum & Minimum \\
\hline $\begin{array}{l}202 \\
203 \\
204 \\
205 \\
206\end{array}$ & $\begin{array}{l}144.2 \\
152.7 \\
156.0 \\
148.1 \\
142.2\end{array}$ & $\begin{array}{l}484.4 \\
510.3 \\
509.6 \\
505.7 \\
493.1\end{array}$ & $\begin{array}{l}30.77 \\
32.54 \\
31.93 \\
32.47 \\
31.75\end{array}$ & $\begin{array}{l}38.83 \\
40.07 \\
40.38 \\
40.66 \\
42.08\end{array}$ & $\begin{array}{l}19.52 \\
23.62 \\
19.16 \\
23.29 \\
16.77\end{array}$ & $\begin{array}{l}34.0 \\
22.3 \\
23.3 \\
17.7 \\
14.6\end{array}$ & $\begin{array}{l}66.2 \\
39.1 \\
55.3 \\
38.4 \\
40.5\end{array}$ & $\begin{array}{r}18.1 \\
11.2 \\
11.0 \\
8.2 \\
3.9\end{array}$ & $\begin{array}{l}1.61 \\
1.92 \\
1.71 \\
1.78 \\
1.43\end{array}$ & $\begin{array}{l}3.85 \\
3.60 \\
4.10 \\
3.14 \\
2.67\end{array}$ & $\begin{array}{r}0.48 \\
.68 \\
.26 \\
.65 \\
.56\end{array}$ \\
\hline $\begin{array}{l}207 \\
208 \\
209 \\
210 \\
211\end{array}$ & $\begin{array}{l}137.5 \\
147.5 \\
145.0 \\
128.9 \\
149.9\end{array}$ & $\begin{array}{l}494.2 \\
492.0 \\
498.2 \\
487.1 \\
511.1\end{array}$ & $\begin{array}{l}30.43 \\
31.98 \\
32.97 \\
33.96 \\
34.15\end{array}$ & $\begin{array}{l}42.81 \\
43.09 \\
43.04 \\
41.25 \\
40.46\end{array}$ & $\begin{array}{l}15.86 \\
15.42 \\
20.47 \\
23.03 \\
28.06\end{array}$ & $\begin{array}{l}20.3 \\
19.9 \\
20.0 \\
24.3 \\
21.7\end{array}$ & $\begin{array}{l}48.8 \\
59.1 \\
43.6 \\
41.2 \\
36.9\end{array}$ & $\begin{array}{r}5.8 \\
6.0 \\
8.8 \\
14.7 \\
13.3\end{array}$ & $\begin{array}{l}1.25 \\
1.50 \\
1.51 \\
2.11 \\
2.47\end{array}$ & $\begin{array}{l}3.00 \\
3.48 \\
3.77 \\
3.82 \\
4.27\end{array}$ & $\begin{array}{l}.42 \\
.42 \\
.27 \\
.47 \\
.92\end{array}$ \\
\hline $\begin{array}{l}212 \\
213 \\
214 \\
215 \\
216\end{array}$ & $\begin{array}{l}148.2 \\
147.8 \\
143.3 \\
145.7 \\
146.6\end{array}$ & $\begin{array}{l}516.5 \\
517.2 \\
512.6 \\
491.5 \\
502.5\end{array}$ & $\begin{array}{l}32.95 \\
33.51 \\
32.39 \\
28.85 \\
28.30\end{array}$ & $\begin{array}{l}40.74 \\
41.06 \\
41.05 \\
41.38 \\
41.27\end{array}$ & $\begin{array}{l}24.35 \\
23.01 \\
19.98 \\
13.30 \\
11.29\end{array}$ & $\begin{array}{l}17.4 \\
14.1 \\
11.8 \\
18.6 \\
15.8\end{array}$ & $\begin{array}{l}30.6 \\
29.2 \\
28.5 \\
51.2 \\
49.9\end{array}$ & $\begin{array}{l}8.5 \\
7.5 \\
3.7 \\
3.5 \\
3.3\end{array}$ & $\begin{array}{l}2.14 \\
2.34 \\
1.83 \\
1.38 \\
1.49\end{array}$ & $\begin{array}{l}3.99 \\
4.01 \\
3.33 \\
3.91 \\
3.49\end{array}$ & $\begin{array}{l}.82 \\
.71 \\
.64 \\
.39 \\
.43\end{array}$ \\
\hline $\begin{array}{l}217 \\
218 \\
219 \\
220 \\
221\end{array}$ & $\begin{array}{l}144.5 \\
140.9 \\
137.7 \\
103.7 \\
135.9\end{array}$ & $\begin{array}{l}520.9 \\
487.0 \\
495.2 \\
445.9 \\
513.4\end{array}$ & $\begin{array}{l}32.00 \\
34.88 \\
33.96 \\
33.25 \\
32.52\end{array}$ & $\begin{array}{l}42.99 \\
44.74 \\
43.03 \\
37.80 \\
38.77\end{array}$ & $\begin{array}{l}15.39 \\
19.78 \\
18.11 \\
29.29 \\
27.01\end{array}$ & $\begin{array}{l}14.6 \\
15.5 \\
19.6 \\
22.0 \\
32.4\end{array}$ & $\begin{array}{l}35.8 \\
39.3 \\
51.4 \\
33.7 \\
42.7\end{array}$ & $\begin{array}{r}5.4 \\
7.7 \\
8.5 \\
15.6 \\
20.8\end{array}$ & $\begin{array}{l}2.16 \\
1.56 \\
2.15 \\
3.74 \\
3.08\end{array}$ & $\begin{array}{l}4.72 \\
2.78 \\
4.71 \\
5.90 \\
4.08\end{array}$ & $\begin{array}{r}.44 \\
.43 \\
.38 \\
1.76 \\
1.91\end{array}$ \\
\hline $\begin{array}{l}222 \\
223 \\
224 \\
225 \\
226\end{array}$ & $\begin{array}{l}128.0 \\
146.1 \\
140.2 \\
135.1 \\
146.4\end{array}$ & $\begin{array}{l}499.5 \\
497.5 \\
519.4 \\
492.3 \\
489.6\end{array}$ & $\begin{array}{l}32.02 \\
33.18 \\
34.25 \\
32.53 \\
31.34\end{array}$ & $\begin{array}{l}39.63 \\
41.87 \\
40.51 \\
40.90 \\
41.65\end{array}$ & $\begin{array}{l}23.54 \\
24.11 \\
27.73 \\
26.38 \\
20.57\end{array}$ & $\begin{array}{l}35.8 \\
29.7 \\
26.6 \\
34.0 \\
36.5\end{array}$ & $\begin{array}{l}64.8 \\
49.0 \\
44.2 \\
45.9 \\
66.5\end{array}$ & $\begin{array}{l}17.8 \\
15.2 \\
17.2 \\
19.1 \\
14.8\end{array}$ & $\begin{array}{r}1.51 \\
1.43 \\
2.59 \\
1.62 \\
.88\end{array}$ & $\begin{array}{l}2.76 \\
2.52 \\
4.85 \\
3.10 \\
1.80\end{array}$ & $\begin{array}{l}.53 \\
.29 \\
.64 \\
.50 \\
.22\end{array}$ \\
\hline $\begin{array}{l}227 \\
228 \\
229 \\
230 \\
231\end{array}$ & $\begin{array}{l}145.0 \\
140.3 \\
108.9 \\
128.2 \\
134.2\end{array}$ & $\begin{array}{l}485.7 \\
488.5 \\
484.1 \\
472.7 \\
455.2\end{array}$ & $\begin{array}{l}31.74 \\
34.87 \\
32.06 \\
32.98 \\
32.30\end{array}$ & $\begin{array}{l}43.57 \\
43.42 \\
38.84 \\
39.09 \\
39.58\end{array}$ & $\begin{array}{l}18.75 \\
21.82 \\
23.84 \\
25.77 \\
21.99\end{array}$ & $\begin{array}{l}31.4 \\
21.1 \\
29.0 \\
28.7 \\
30.6\end{array}$ & $\begin{array}{l}63.3 \\
42.8 \\
38.9 \\
48.8 \\
56.8\end{array}$ & $\begin{array}{r}9.6 \\
9.6 \\
18.4 \\
16.2 \\
17.6\end{array}$ & $\begin{array}{r}.84 \\
2.21 \\
2.84 \\
2.21 \\
1.85\end{array}$ & $\begin{array}{l}1.60 \\
4.23 \\
6.23 \\
3.60 \\
3.69\end{array}$ & $\begin{array}{l}.21 \\
.40 \\
.62 \\
.47 \\
.48\end{array}$ \\
\hline $\begin{array}{l}232 \\
233 \\
234 \\
235 \\
236\end{array}$ & $\begin{array}{l}137.5 \\
121.0 \\
130.5 \\
136.7 \\
135.3\end{array}$ & $\begin{array}{l}476.3 \\
468.4 \\
492.7 \\
486.8 \\
473.6\end{array}$ & $\begin{array}{l}32.17 \\
31.67 \\
29.38 \\
25.77 \\
27.81\end{array}$ & $\begin{array}{l}40.45 \\
38.56 \\
37.00 \\
38.24 \\
40.54\end{array}$ & $\begin{array}{l}19.74 \\
23.75 \\
22.20 \\
11.40 \\
11.66\end{array}$ & $\begin{array}{l}28.0 \\
13.5 \\
12.8 \\
14.4 \\
15.5\end{array}$ & $\begin{array}{l}63.4 \\
27.5 \\
26.5 \\
31.5 \\
43.5\end{array}$ & $\begin{array}{r}13.7 \\
3.5 \\
3.3 \\
3.2 \\
3.3\end{array}$ & $\begin{array}{l}1.93 \\
3.35 \\
2.50 \\
1.53 \\
1.63\end{array}$ & $\begin{array}{l}4.05 \\
5.28 \\
3.89 \\
3.53 \\
3.57\end{array}$ & $\begin{array}{r}.47 \\
1.17 \\
.76 \\
.40 \\
.47\end{array}$ \\
\hline
\end{tabular}


Table 3. Daily mean, maximum, and minimum micrometeorological data measured by micromet station at Rogers Spring Site 1, 1994-Continued

\begin{tabular}{|c|c|c|c|c|c|c|c|c|c|c|c|c|}
\hline & \multirow{2}{*}{$\begin{array}{l}\text { Calendar } \\
\text { day }\end{array}$} & \multicolumn{2}{|c|}{$\begin{array}{l}\text { Net radiation, } \\
\text { in watts per } \\
\text { square meter }\end{array}$} & \multicolumn{3}{|c|}{$\begin{array}{l}\text { Air temperature at } 0.7 \text { meter, } \\
\text { in degrees Celsius }\end{array}$} & \multicolumn{3}{|c|}{$\begin{array}{l}\text { Relative humidity at } 0.7 \text { meter, } \\
\text { in percent }\end{array}$} & \multicolumn{3}{|c|}{$\begin{array}{l}\text { Windspeed at } 0.7 \text { meter, } \\
\text { in meters per second }\end{array}$} \\
\hline & & Mean & Maximum & Mean & Maximum & Minimum & Mean & Maximum & Minimum & Mean & Maximum & Minimum \\
\hline & $\begin{array}{l}237 \\
238 \\
239 \\
240 \\
241\end{array}$ & $\begin{array}{l}128.5 \\
126.4 \\
118.5 \\
131.7 \\
131.2\end{array}$ & $\begin{array}{l}469.3 \\
489.1 \\
479.1 \\
479.4 \\
478.0\end{array}$ & $\begin{array}{l}31.86 \\
31.48 \\
30.68 \\
31.65 \\
28.87\end{array}$ & $\begin{array}{l}40.22 \\
40.12 \\
38.13 \\
39.71 \\
38.92\end{array}$ & $\begin{array}{l}21.06 \\
19.29 \\
24.70 \\
21.94 \\
14.30\end{array}$ & $\begin{array}{l}10.2 \\
18.0 \\
31.9 \\
18.9 \\
14.8\end{array}$ & $\begin{array}{l}23.3 \\
35.5 \\
63.1 \\
34.4 \\
39.1\end{array}$ & $\begin{array}{r}4.9 \\
5.0 \\
12.2 \\
11.5 \\
3.2\end{array}$ & $\begin{array}{l}2.46 \\
2.50 \\
1.90 \\
2.04 \\
1.72\end{array}$ & $\begin{array}{l}4.92 \\
4.60 \\
3.44 \\
4.00 \\
3.19\end{array}$ & $\begin{array}{r}0.46 \\
.49 \\
.79 \\
.67 \\
.41\end{array}$ \\
\hline & $\begin{array}{l}242 \\
243 \\
244 \\
245 \\
246\end{array}$ & $\begin{array}{l}131.1 \\
115.6 \\
122.9 \\
106.7 \\
128.5\end{array}$ & $\begin{array}{l}479.8 \\
459.1 \\
462.4 \\
448.2 \\
475.3\end{array}$ & $\begin{array}{l}24.57 \\
26.72 \\
28.38 \\
28.53 \\
24.41\end{array}$ & $\begin{array}{l}38.34 \\
37.58 \\
36.68 \\
36.09 \\
35.44\end{array}$ & $\begin{array}{r}9.12 \\
10.62 \\
16.88 \\
16.05 \\
11.23\end{array}$ & $\begin{array}{l}20.7 \\
16.4 \\
14.7 \\
12.6 \\
19.6\end{array}$ & $\begin{array}{l}53.3 \\
51.4 \\
31.9 \\
33.1 \\
40.7\end{array}$ & $\begin{array}{l}3.4 \\
4.2 \\
6.0 \\
3.3 \\
6.2\end{array}$ & $\begin{array}{l}1.36 \\
1.91 \\
2.52 \\
3.22 \\
1.43\end{array}$ & $\begin{array}{l}3.53 \\
4.11 \\
4.37 \\
5.21 \\
2.93\end{array}$ & $\begin{array}{l}.36 \\
.36 \\
.61 \\
.56 \\
.49\end{array}$ \\
\hline & $\begin{array}{l}247 \\
248 \\
249 \\
250 \\
251\end{array}$ & $\begin{array}{l}131.4 \\
131.5 \\
128.2 \\
124.0 \\
113.2\end{array}$ & $\begin{array}{l}466.9 \\
466.8 \\
470.8 \\
466.0 \\
438.4\end{array}$ & $\begin{array}{l}24.36 \\
26.90 \\
29.94 \\
30.56 \\
30.71\end{array}$ & $\begin{array}{l}37.25 \\
38.61 \\
39.06 \\
39.24 \\
38.16\end{array}$ & $\begin{array}{r}9.64 \\
14.34 \\
17.33 \\
21.48 \\
19.21\end{array}$ & $\begin{array}{l}28.7 \\
34.4 \\
27.9 \\
18.2 \\
13.5\end{array}$ & $\begin{array}{l}54.9 \\
61.9 \\
60.0 \\
34.5 \\
33.5\end{array}$ & $\begin{array}{r}12.2 \\
14.7 \\
10.8 \\
6.2 \\
3.8\end{array}$ & $\begin{array}{l}1.06 \\
1.06 \\
1.48 \\
1.81 \\
2.85\end{array}$ & $\begin{array}{l}2.61 \\
2.67 \\
3.17 \\
3.49 \\
5.28\end{array}$ & $\begin{array}{l}.30 \\
.31 \\
.40 \\
.48 \\
.62\end{array}$ \\
\hline & $\begin{array}{l}252 \\
253 \\
254 \\
255 \\
256\end{array}$ & $\begin{array}{r}81.3 \\
82.4 \\
99.0 \\
104.3 \\
115.8\end{array}$ & $\begin{array}{l}418.2 \\
409.7 \\
409.9 \\
455.9 \\
453.6\end{array}$ & $\begin{array}{l}29.18 \\
30.37 \\
26.43 \\
23.04 \\
17.61\end{array}$ & $\begin{array}{l}36.22 \\
35.90 \\
32.56 \\
28.25 \\
27.34\end{array}$ & $\begin{array}{r}16.05 \\
25.47 \\
20.66 \\
17.56 \\
7.50\end{array}$ & $\begin{array}{r}14.0 \\
9.8 \\
13.2 \\
21.6 \\
36.9\end{array}$ & $\begin{array}{l}37.4 \\
16.1 \\
25.0 \\
33.8 \\
63.2\end{array}$ & $\begin{array}{r}5.9 \\
3.3 \\
5.6 \\
9.5 \\
14.9\end{array}$ & $\begin{array}{l}2.37 \\
5.04 \\
4.61 \\
3.98 \\
1.28\end{array}$ & $\begin{array}{l}4.79 \\
6.47 \\
6.26 \\
5.90 \\
2.16\end{array}$ & $\begin{array}{r}.34 \\
3.09 \\
3.16 \\
1.93 \\
.46\end{array}$ \\
\hline & $\begin{array}{l}257 \\
258 \\
259 \\
260 \\
261\end{array}$ & $\begin{array}{r}117.0 \\
115.1 \\
97.8 \\
92.8 \\
89.8\end{array}$ & $\begin{array}{l}451.5 \\
449.7 \\
452.2 \\
397.2 \\
397.4\end{array}$ & $\begin{array}{l}19.87 \\
22.06 \\
22.15 \\
22.33 \\
22.98\end{array}$ & $\begin{array}{l}31.23 \\
34.54 \\
34.62 \\
35.16 \\
36.06\end{array}$ & $\begin{array}{r}9.39 \\
8.77 \\
7.75 \\
10.48 \\
11.28\end{array}$ & $\begin{array}{l}31.3 \\
24.2 \\
27.6 \\
24.0 \\
30.0\end{array}$ & $\begin{array}{l}57.3 \\
45.7 \\
57.2 \\
40.4 \\
54.1\end{array}$ & $\begin{array}{r}11.4 \\
7.4 \\
7.9 \\
7.8 \\
11.0\end{array}$ & $\begin{array}{r}1.03 \\
1.14 \\
.92 \\
1.24 \\
.82\end{array}$ & $\begin{array}{l}1.54 \\
3.17 \\
2.49 \\
3.79 \\
1.71\end{array}$ & $\begin{array}{l}.36 \\
.38 \\
.20 \\
.36 \\
.34\end{array}$ \\
\hline & $\begin{array}{l}262 \\
263 \\
264 \\
265 \\
266\end{array}$ & $\begin{array}{r}82.3 \\
107.3 \\
114.5 \\
109.0 \\
112.3\end{array}$ & $\begin{array}{l}468.3 \\
468.1 \\
441.9 \\
436.2 \\
432.0\end{array}$ & $\begin{array}{l}22.70 \\
24.88 \\
26.12 \\
26.31 \\
26.60\end{array}$ & $\begin{array}{l}31.25 \\
33.59 \\
37.10 \\
38.99 \\
42.87\end{array}$ & $\begin{array}{l}13.66 \\
15.87 \\
13.67 \\
11.31 \\
12.42\end{array}$ & $\begin{array}{l}38.8 \\
36.5 \\
36.1 \\
34.4 \\
30.0\end{array}$ & $\begin{array}{l}65.1 \\
61.3 \\
57.7 \\
64.9 \\
53.5\end{array}$ & $\begin{array}{l}24.1 \\
20.4 \\
13.8 \\
13.7 \\
12.9\end{array}$ & $\begin{array}{r}1.81 \\
1.60 \\
1.06 \\
.91 \\
.97\end{array}$ & $\begin{array}{l}3.65 \\
2.58 \\
2.35 \\
1.72 \\
1.81\end{array}$ & $\begin{array}{l}.40 \\
.75 \\
.31 \\
.44 \\
.29\end{array}$ \\
\hline & $\begin{array}{l}267 \\
268 \\
269 \\
270 \\
271\end{array}$ & $\begin{array}{r}86.2 \\
111.0 \\
103.2 \\
99.7 \\
62.7\end{array}$ & $\begin{array}{l}403.9 \\
434.4 \\
419.9 \\
413.9 \\
281.0\end{array}$ & $\begin{array}{l}29.69 \\
26.74 \\
26.20 \\
30.08 \\
30.50\end{array}$ & $\begin{array}{l}39.50 \\
41.63 \\
40.93 \\
45.18 \\
37.47\end{array}$ & $\begin{array}{l}19.61 \\
14.74 \\
12.67 \\
13.72 \\
19.21\end{array}$ & $\begin{array}{l}37.9 \\
38.4 \\
32.7 \\
34.3 \\
39.2\end{array}$ & $\begin{array}{l}59.6 \\
64.5 \\
55.3 \\
55.8 \\
68.2\end{array}$ & $\begin{array}{l}21.5 \\
16.0 \\
12.1 \\
16.1 \\
24.1\end{array}$ & $\begin{array}{r}1.35 \\
1.07 \\
.91 \\
1.37 \\
1.48\end{array}$ & $\begin{array}{l}3.33 \\
2.70 \\
1.75 \\
3.72 \\
3.17\end{array}$ & $\begin{array}{l}.44 \\
.23 \\
.38 \\
.46 \\
.39\end{array}$ \\
\hline
\end{tabular}


今)

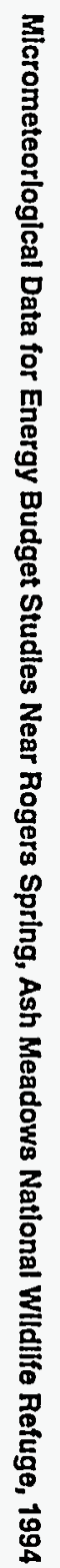

Table 3. Daily mean, maximum, and minimum micrometeorological data measured by micromet station at Rogers Spring Site 1, 1994-Continued

\begin{tabular}{|c|c|c|c|c|c|c|c|c|c|c|c|}
\hline \multirow[t]{2}{*}{$\begin{array}{l}\text { Calendar } \\
\text { day }\end{array}$} & \multicolumn{2}{|c|}{$\begin{array}{l}\text { Net radiation, } \\
\text { in watts per } \\
\text { square meter }\end{array}$} & \multicolumn{3}{|c|}{$\begin{array}{l}\text { Air temperature at } 0.7 \text { meter, } \\
\text { in degrees Celsius }\end{array}$} & \multicolumn{3}{|c|}{$\begin{array}{l}\text { Relative humidity at } 0.7 \text { meter, } \\
\text { in percent }\end{array}$} & \multicolumn{3}{|c|}{$\begin{array}{l}\text { Windspeed at } 0.7 \text { meter, } \\
\text { in meters per second }\end{array}$} \\
\hline & Mean & Maximum & Mean & Maximum & Minimum & Mean & Maximum & Minimum & Mean & Maximum & Minimum \\
\hline $\begin{array}{l}272 \\
273 \\
274 \\
275 \\
276\end{array}$ & $\begin{array}{l}51.2 \\
18.5 \\
27.5 \\
39.2 \\
57.8\end{array}$ & $\begin{array}{l}251.1 \\
206.4 \\
224.1 \\
281.2 \\
369.8\end{array}$ & $\begin{array}{l}28.07 \\
21.55 \\
21.74 \\
20.16 \\
16.40\end{array}$ & $\begin{array}{l}34.56 \\
27.78 \\
29.05 \\
23.74 \\
24.29\end{array}$ & $\begin{array}{r}20.28 \\
16.69 \\
16.15 \\
16.94 \\
6.62\end{array}$ & $\begin{array}{l}49.8 \\
56.0 \\
31.2 \\
42.2 \\
52.0\end{array}$ & $\begin{array}{l}89.2 \\
62.9 \\
46.6 \\
54.1 \\
73.8\end{array}$ & $\begin{array}{l}14.7 \\
39.7 \\
13.8 \\
30.9 \\
30.6\end{array}$ & $\begin{array}{l}2.57 \\
1.74 \\
1.54 \\
3.85 \\
2.33\end{array}$ & $\begin{array}{l}5.72 \\
2.76 \\
3.58 \\
5.14 \\
4.89\end{array}$ & $\begin{array}{r}0.58 \\
.84 \\
.24 \\
2.42 \\
.33\end{array}$ \\
\hline $\begin{array}{l}277 \\
278 \\
279 \\
280 \\
281\end{array}$ & $\begin{array}{l}39.2 \\
57.8 \\
62.7 \\
54.4 \\
48.9\end{array}$ & $\begin{array}{l}281.2 \\
369.8 \\
363.3 \\
372.8 \\
352.6\end{array}$ & $\begin{array}{l}20.16 \\
16.40 \\
17.34 \\
21.12 \\
20.05\end{array}$ & $\begin{array}{l}23.74 \\
24.29 \\
28.41 \\
28.80 \\
29.63\end{array}$ & $\begin{array}{r}16.94 \\
6.62 \\
3.67 \\
10.13 \\
8.97\end{array}$ & $\begin{array}{l}42.2 \\
52.0 \\
45.6 \\
32.0 \\
28.3\end{array}$ & $\begin{array}{l}54.1 \\
73.8 \\
78.4 \\
66.7 \\
52.1\end{array}$ & $\begin{array}{l}30.9 \\
30.6 \\
20.8 \\
12.7 \\
12.6\end{array}$ & $\begin{array}{r}3.85 \\
2.33 \\
.90 \\
1.57 \\
1.45\end{array}$ & $\begin{array}{l}5.14 \\
4.89 \\
1.56 \\
2.73 \\
2.98\end{array}$ & $\begin{array}{r}2.42 \\
.33 \\
.44 \\
.61 \\
.45\end{array}$ \\
\hline $\begin{array}{l}282 \\
283 \\
284 \\
285 \\
286\end{array}$ & $\begin{array}{l}56.0 \\
- \\
-- \\
- \\
--\end{array}$ & $\begin{array}{c}349.9 \\
-- \\
-- \\
-- \\
--\end{array}$ & $\begin{array}{l}17.64 \\
- \\
- \\
-- \\
--\end{array}$ & $\begin{array}{l}31.39 \\
-- \\
-- \\
-- \\
--\end{array}$ & $\begin{array}{l}4.72 \\
-- \\
-- \\
- \\
-\end{array}$ & $\begin{array}{l}33.1 \\
- \\
- \\
- \\
-\end{array}$ & $\begin{array}{l}62.7 \\
- \\
- \\
- \\
--\end{array}$ & $\begin{array}{l}10.1 \\
-- \\
-- \\
-- \\
--\end{array}$ & $\begin{array}{l}.80 \\
-- \\
- \\
-- \\
--\end{array}$ & $\begin{array}{l}1.82 \\
-- \\
- \\
- \\
-\end{array}$ & $\begin{array}{l}.27 \\
\ldots \\
-- \\
-- \\
--\end{array}$ \\
\hline $\begin{array}{l}287 \\
288 \\
289 \\
290 \\
291\end{array}$ & $\begin{array}{c}29.8 \\
44.4 \\
43.1 \\
-- \\
--\end{array}$ & $\begin{array}{c}294.6 \\
321.4 \\
334.1 \\
-- \\
--\end{array}$ & $\begin{array}{l}19.53 \\
14.49 \\
27.87 \\
-- \\
--\end{array}$ & $\begin{array}{l}25.17 \\
20.26 \\
59.15 \\
-- \\
--\end{array}$ & $\begin{array}{l}14.41 \\
7.24 \\
3.75 \\
-- \\
--\end{array}$ & $\begin{array}{l}41.3 \\
36.8 \\
51.0 \\
-- \\
--\end{array}$ & $\begin{array}{c}56.4 \\
59.3 \\
65.9 \\
-- \\
--\end{array}$ & $\begin{array}{l}31.3 \\
20.6 \\
31.0 \\
-- \\
-\end{array}$ & $\begin{array}{l}1.80 \\
2.50 \\
1.50 \\
-. \\
-\end{array}$ & $\begin{array}{l}3.16 \\
4.46 \\
2.69 \\
-- \\
--\end{array}$ & $\begin{array}{l}.57 \\
.49 \\
.26 \\
-- \\
--\end{array}$ \\
\hline $\begin{array}{l}292 \\
293 \\
294 \\
295 \\
296\end{array}$ & $\begin{array}{l}46.0 \\
43.2 \\
43.9 \\
29.5 \\
40.8\end{array}$ & $\begin{array}{l}319.3 \\
305.7 \\
307.4 \\
247.4 \\
249.8\end{array}$ & $\begin{array}{l}12.56 \\
12.85 \\
12.80 \\
13.08 \\
14.97\end{array}$ & $\begin{array}{l}25.38 \\
26.07 \\
27.48 \\
26.91 \\
26.90\end{array}$ & $\begin{array}{r}2.29 \\
1.64 \\
.27 \\
1.41 \\
1.16\end{array}$ & $\begin{array}{l}34.0 \\
33.0 \\
32.7 \\
31.5 \\
28.4\end{array}$ & $\begin{array}{l}53.2 \\
53.5 \\
63.8 \\
57.9 \\
61.4\end{array}$ & $\begin{array}{l}14.0 \\
14.5 \\
11.7 \\
11.3 \\
12.3\end{array}$ & $\begin{array}{l}.75 \\
.66 \\
.62 \\
.54 \\
.78\end{array}$ & $\begin{array}{l}1.62 \\
1.19 \\
1.24 \\
1.32 \\
1.77\end{array}$ & $\begin{array}{l}.09 \\
.07 \\
.07 \\
.07 \\
.19\end{array}$ \\
\hline $\begin{array}{l}297 \\
298 \\
299 \\
300 \\
301\end{array}$ & $\begin{array}{l}44.5 \\
42.6 \\
39.0 \\
35.5 \\
37.3\end{array}$ & $\begin{array}{l}295.7 \\
297.7 \\
291.1 \\
318.7 \\
291.3\end{array}$ & $\begin{array}{l}16.35 \\
15.39 \\
15.07 \\
15.23 \\
14.02\end{array}$ & $\begin{array}{l}27.50 \\
29.03 \\
28.34 \\
28.33 \\
27.54\end{array}$ & $\begin{array}{l}5.33 \\
5.09 \\
4.36 \\
4.45 \\
3.56\end{array}$ & $\begin{array}{l}31.8 \\
37.1 \\
35.8 \\
34.7 \\
36.4\end{array}$ & $\begin{array}{l}54.5 \\
56.1 \\
55.2 \\
52.9 \\
63.2\end{array}$ & $\begin{array}{l}14.0 \\
15.3 \\
15.0 \\
16.1 \\
14.4\end{array}$ & $\begin{array}{l}.73 \\
.60 \\
.66 \\
.69 \\
.67\end{array}$ & $\begin{array}{l}1.50 \\
1.41 \\
1.16 \\
1.88 \\
1.34\end{array}$ & $\begin{array}{l}.09 \\
.07 \\
.07 \\
.15 \\
.07\end{array}$ \\
\hline $\begin{array}{l}302 \\
303 \\
304 \\
305 \\
306\end{array}$ & $\begin{array}{r}33.5 \\
23.3 \\
30.9 \\
19.7 \\
5.5\end{array}$ & $\begin{array}{l}291.5 \\
292.7 \\
276.9 \\
272.1 \\
145.1\end{array}$ & $\begin{array}{l}14.84 \\
14.59 \\
11.44 \\
15.85 \\
15.02\end{array}$ & $\begin{array}{l}26.79 \\
24.26 \\
25.93 \\
26.92 \\
19.43\end{array}$ & $\begin{array}{r}2.61 \\
4.14 \\
-1.04 \\
1.36 \\
8.78\end{array}$ & $\begin{array}{l}31.8 \\
21.2 \\
20.6 \\
17.4 \\
38.6\end{array}$ & $\begin{array}{l}55.5 \\
32.5 \\
51.9 \\
29.4 \\
56.0\end{array}$ & $\begin{array}{r}16.9 \\
9.9 \\
3.3 \\
8.0 \\
25.6\end{array}$ & $\begin{array}{r}1.20 \\
1.47 \\
.61 \\
2.51 \\
3.15\end{array}$ & $\begin{array}{l}2.52 \\
2.74 \\
1.37 \\
5.23 \\
4.91\end{array}$ & $\begin{array}{l}.32 \\
.44 \\
.07 \\
.35 \\
.23\end{array}$ \\
\hline
\end{tabular}


Table 3. Daily mean, maximum, and minimum micrometeorological data measured by micromet station at Rogers Spring Site 1, 1994 - Continued

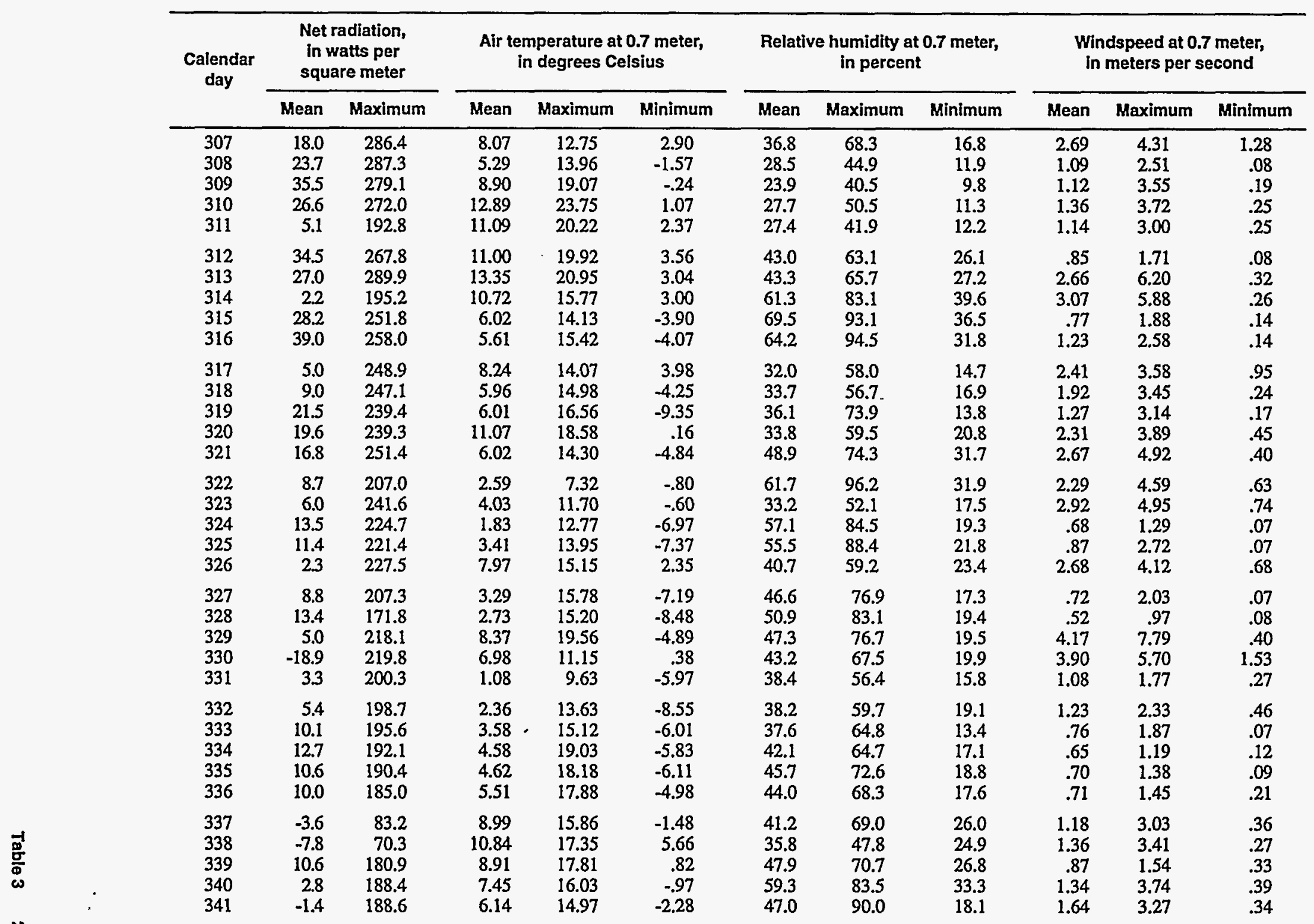


Table 3. Daily mean, maximum, and minimum micrometeorological data measured by micromet station at Rogers Spring Site 1, 1994-Continued

\begin{tabular}{|c|c|c|c|c|c|c|c|c|c|c|c|}
\hline \multirow{2}{*}{$\begin{array}{l}\text { Calendar } \\
\text { day }\end{array}$} & \multicolumn{2}{|c|}{$\begin{array}{l}\text { Net radiation, } \\
\text { in watts per } \\
\text { square meter }\end{array}$} & \multicolumn{3}{|c|}{$\begin{array}{l}\text { Air temperature at } 0.7 \text { meter, } \\
\text { in degrees Celsius }\end{array}$} & \multicolumn{3}{|c|}{$\begin{array}{l}\text { Relative humidity at } 0.7 \text { meter, } \\
\text { in percent }\end{array}$} & \multicolumn{3}{|c|}{$\begin{array}{l}\text { Windspeed at } 0.7 \text { meter, } \\
\text { in meters per second }\end{array}$} \\
\hline & Mean & Maximum & Mean & Maximum & Minimum & Mean & Maximum & Minimum & Mean & Maximum & Minimum \\
\hline $\begin{array}{l}342 \\
343 \\
344 \\
345 \\
346\end{array}$ & $\begin{array}{r}-8.7 \\
-0.4 \\
-6.4 \\
1.2 \\
10.2\end{array}$ & $\begin{array}{l}184.5 \\
179.5 \\
133.7 \\
131.3 \\
182.2\end{array}$ & $\begin{array}{r}4.12 \\
.03 \\
-1.36 \\
-1.13 \\
7.27\end{array}$ & $\begin{array}{r}10.93 \\
13.12 \\
9.93 \\
9.72 \\
16.11\end{array}$ & $\begin{array}{r}-6.70 \\
-9.74 \\
-10.60 \\
-10.42 \\
-4.03\end{array}$ & $\begin{array}{l}26.8 \\
32.8 \\
40.0 \\
39.3 \\
30.5\end{array}$ & $\begin{array}{l}48.8 \\
57.4 \\
68.5 \\
64.0 \\
52.8\end{array}$ & $\begin{array}{r}11.3 \\
8.8 \\
17.8 \\
17.0 \\
15.3\end{array}$ & $\begin{array}{r}2.39 \\
1.10 \\
.81 \\
.74 \\
2.69\end{array}$ & $\begin{array}{l}4.04 \\
2.62 \\
1.54 \\
1.39 \\
5.12\end{array}$ & $\begin{array}{r}0.23 \\
.26 \\
.30 \\
.33 \\
.33\end{array}$ \\
\hline $\begin{array}{l}347 \\
348 \\
349 \\
350 \\
351\end{array}$ & $\begin{array}{r}-11.0 \\
-3.6 \\
5.5 \\
7.6 \\
7.6\end{array}$ & $\begin{array}{l}125.7 \\
124.1 \\
171.2 \\
171.7 \\
175.5\end{array}$ & $\begin{array}{r}6.44 \\
.79 \\
1.18 \\
2.20 \\
4.06\end{array}$ & $\begin{array}{l}12.03 \\
10.39 \\
12.90 \\
15.54 \\
18.55\end{array}$ & $\begin{array}{l}-3.94 \\
-7.85 \\
-7.83 \\
-7.73 \\
-5.89\end{array}$ & $\begin{array}{l}64.8 \\
63.9 \\
57.7 \\
53.3 \\
49.7\end{array}$ & $\begin{array}{l}91.6 \\
91.0 \\
82.0 \\
77.8 \\
76.0\end{array}$ & $\begin{array}{l}38.5 \\
31.7 \\
28.1 \\
24.5 \\
22.2\end{array}$ & $\begin{array}{r}2.04 \\
.68 \\
.66 \\
.71 \\
.80\end{array}$ & $\begin{array}{l}5.11 \\
1.43 \\
1.23 \\
1.52 \\
1.20\end{array}$ & $\begin{array}{l}.20 \\
.18 \\
.12 \\
.23 \\
.43\end{array}$ \\
\hline $\begin{array}{l}352 \\
353 \\
354 \\
355 \\
356\end{array}$ & $\begin{array}{r}6.4 \\
3.1 \\
2.0 \\
9.3 \\
5\end{array}$ & $\begin{array}{r}172.9 \\
169.0 \\
165.6 \\
165.2 \\
81.2\end{array}$ & $\begin{array}{r}3.41 \\
5.24 \\
4.65 \\
5.22 \\
10.22\end{array}$ & $\begin{array}{l}17.11 \\
18.87 \\
19.79 \\
20.39 \\
18.33\end{array}$ & $\begin{array}{r}-6.28 \\
-5.10 \\
-7.12 \\
-6.53 \\
.05\end{array}$ & $\begin{array}{l}50.5 \\
43.3 \\
41.4 \\
39.0 \\
31.3\end{array}$ & $\begin{array}{l}73.8 \\
75.7 \\
68.6 \\
69.1 \\
51.5\end{array}$ & $\begin{array}{l}22.7 \\
13.9 \\
14.4 \\
12.3 \\
14.4\end{array}$ & $\begin{array}{r}.84 \\
.81 \\
.80 \\
.68 \\
1.28\end{array}$ & $\begin{array}{r}1.34 \\
1.25 \\
1.18 \\
.96 \\
3.60\end{array}$ & $\begin{array}{l}.34 \\
.23 \\
.52 \\
.23 \\
.31\end{array}$ \\
\hline $\begin{array}{l}357 \\
358 \\
359 \\
360 \\
361\end{array}$ & $\begin{array}{r}12.3 \\
6.2 \\
64.1 \\
13.6 \\
18.3\end{array}$ & $\begin{array}{r}135.5 \\
73.8 \\
265.2 \\
267.6 \\
232.9\end{array}$ & $\begin{array}{r}12.81 \\
10.81 \\
10.58 \\
10.72 \\
7.90\end{array}$ & $\begin{array}{l}16.30 \\
12.59 \\
14.79 \\
18.01 \\
16.71\end{array}$ & $\begin{array}{r}10.82 \\
9.35 \\
7.76 \\
6.09 \\
-.32\end{array}$ & $\begin{array}{l}53.5 \\
87.1 \\
78.7 \\
59.6 \\
72.4\end{array}$ & $\begin{array}{l}68.3 \\
97.8 \\
98.6 \\
74.3 \\
94.2\end{array}$ & $\begin{array}{l}35.8 \\
69.5 \\
60.4 \\
35.8 \\
40.0\end{array}$ & $\begin{array}{r}1.44 \\
1.80 \\
2.01 \\
2.18 \\
.75\end{array}$ & $\begin{array}{l}4.17 \\
3.82 \\
3.58 \\
3.66 \\
1.74\end{array}$ & $\begin{array}{l}.27 \\
.36 \\
.55 \\
.68 \\
.23\end{array}$ \\
\hline $\begin{array}{l}362 \\
363 \\
364 \\
365\end{array}$ & $\begin{array}{l}28.2 \\
11.4 \\
11.4 \\
29.3\end{array}$ & $\begin{array}{l}236.7 \\
151.5 \\
246.0 \\
243.5\end{array}$ & $\begin{array}{l}9.53 \\
7.01 \\
5.82 \\
2.87\end{array}$ & $\begin{array}{l}16.85 \\
10.51 \\
13.47 \\
11.61\end{array}$ & $\begin{array}{r}3.72 \\
5.07 \\
-2.26 \\
-5.01\end{array}$ & $\begin{array}{l}70.7 \\
83.3 \\
63.0 \\
76.3\end{array}$ & $\begin{array}{l}94.0 \\
95.2 \\
93.8 \\
93.4\end{array}$ & $\begin{array}{l}39.9 \\
68.6 \\
29.4 \\
44.5\end{array}$ & $\begin{array}{r}2.28 \\
1.10 \\
1.73 \\
.49\end{array}$ & $\begin{array}{r}4.84 \\
2.84 \\
3.11 \\
.84\end{array}$ & $\begin{array}{l}.31 \\
.29 \\
.24 \\
.20\end{array}$ \\
\hline
\end{tabular}


Table 4. Daily mean net radiation, air temperature, dew-point temperature, vapor pressure, soil heat flux, and soil temperature change measured by energy-budget station at Rogers Spring Site 2, 1994

$[-$, no data available $]$

\begin{tabular}{|c|c|c|c|c|c|c|c|c|c|c|c|}
\hline \multirow[t]{2}{*}{$\begin{array}{l}\text { Calendar } \\
\text { day }\end{array}$} & \multirow{2}{*}{$\begin{array}{l}\text { Net radiation, } \\
\text { in watts per } \\
\text { square meter - }\end{array}$} & \multicolumn{2}{|c|}{$\begin{array}{l}\text { Air temperature, } \\
\text { in degrees Celsius }\end{array}$} & \multicolumn{3}{|c|}{$\begin{array}{l}\text { Dew-point temperature, } \\
\text { in degrees Celsius }\end{array}$} & \multicolumn{2}{|c|}{$\begin{array}{l}\text { Vapor pressure, } \\
\text { in kilopascals }\end{array}$} & \multicolumn{2}{|c|}{$\begin{array}{l}\text { Soil heat flux, } \\
\text { in watts per } \\
\text { square meter }\end{array}$} & \multirow{2}{*}{$\begin{array}{l}\text { Difference in soil } \\
\text { temperature over } \\
\text { time, in degress } \\
\text { Celsius }\end{array}$} \\
\hline & & 1.25 meters & 2.25 meters & 1.25 meters & 2.25 meters & & 1.25 meters & 2.25 meters & Plate 1 & Plate 2 & \\
\hline $\begin{array}{l}75 \\
76 \\
77 \\
78 \\
79\end{array}$ & $\begin{array}{r}75.2 \\
91.5 \\
97.2 \\
65.9 \\
109.4\end{array}$ & $\begin{array}{l}22.32 \\
19.12 \\
19.47 \\
15.69 \\
13.23\end{array}$ & $\begin{array}{l}22.38 \\
19.32 \\
19.44 \\
15.69 \\
13.45\end{array}$ & $\begin{array}{l}-6.19 \\
-6.14 \\
-3.16 \\
-2.41 \\
-3.36\end{array}$ & $\begin{array}{l}-6.27 \\
-6.12 \\
-2.95 \\
-2.35 \\
-3.16\end{array}$ & & $\begin{array}{r}0.3877 \\
.3973 \\
.4910 \\
.5161 \\
.4822\end{array}$ & $\begin{array}{r}0.3851 \\
.3961 \\
.4980 \\
.5188 \\
.4895\end{array}$ & $\begin{array}{r}6.9 \\
5.5 \\
6.3 \\
3.8 \\
-2.2\end{array}$ & $\begin{array}{r}14.0 \\
12.6 \\
14.4 \\
8.6 \\
.8\end{array}$ & $\begin{array}{r}0.003 \\
.003 \\
.007 \\
-.005 \\
-.017\end{array}$ \\
\hline $\begin{array}{l}80 \\
81 \\
82 \\
83 \\
84\end{array}$ & $\begin{array}{r}102.0 \\
95.3 \\
103.7 \\
80.1 \\
112.2\end{array}$ & $\begin{array}{r}15.28 \\
13.35 \\
10.28 \\
12.89 \\
9.74\end{array}$ & $\begin{array}{r}15.37 \\
13.52 \\
10.29 \\
12.97 \\
9.72\end{array}$ & $\begin{array}{r}-.33 \\
-3.14 \\
-8.12 \\
-4.89 \\
-3.91\end{array}$ & $\begin{array}{l}-.28 \\
-3.29 \\
-8.35 \\
-4.70 \\
-3.57\end{array}$ & $\cdot$ & $\begin{array}{l}.6495 \\
.5147 \\
.3485 \\
.4336 \\
.4671\end{array}$ & $\begin{array}{l}.6482 \\
.5105 \\
.3399 \\
.4385 \\
.4775\end{array}$ & $\begin{array}{r}1.6 \\
-7.3 \\
-5.5 \\
.5 \\
-4.2\end{array}$ & $\begin{array}{r}5.9 \\
-8.6 \\
-5.2 \\
3.0 \\
-2.4\end{array}$ & $\begin{array}{r}.025 \\
-.052 \\
.026 \\
.001 \\
. .025\end{array}$ \\
\hline $\begin{array}{l}85 \\
86 \\
87 \\
88 \\
89\end{array}$ & $\begin{array}{l}109.1 \\
106.5 \\
100.5 \\
112.2 \\
111.7\end{array}$ & $\begin{array}{l}12.36 \\
15.12 \\
15.77 \\
16.19 \\
18.34\end{array}$ & $\begin{array}{l}12.36 \\
15.49 \\
15.98 \\
16.61 \\
18.67\end{array}$ & $\begin{array}{l}-2.59 \\
-4.67 \\
-5.34 \\
-5.31 \\
-3.97\end{array}$ & $\begin{array}{l}-2.53 \\
-5.02 \\
-5.49 \\
-5.40 \\
-4.09\end{array}$ & & $\begin{array}{l}.5351 \\
.4635 \\
.4425 \\
.4452 \\
.4814\end{array}$ & $\begin{array}{l}.5321 \\
.4466 \\
.4325 \\
.4370 \\
.4723\end{array}$ & $\begin{array}{r}-8.4 \\
-.3 \\
1.3 \\
4.3 \\
6.6\end{array}$ & $\begin{array}{r}-5.5 \\
2.8 \\
6.5 \\
10.2 \\
13.1\end{array}$ & $\begin{array}{l}.016 \\
.003 \\
.022 \\
.014 \\
.003\end{array}$ \\
\hline $\begin{array}{l}90 \\
91 \\
92 \\
93 \\
94\end{array}$ & $\begin{array}{r}117.3 \\
121.6 \\
105.2 \\
66.8 \\
114.0\end{array}$ & $\begin{array}{l}17.67 \\
16.28 \\
18.02 \\
19.81 \\
15.71\end{array}$ & $\begin{array}{l}18.13 \\
16.74 \\
18.36 \\
19.84 \\
15.67\end{array}$ & $\begin{array}{l}-4.42 \\
-5.61 \\
-4.87 \\
-2.10 \\
-4.78\end{array}$ & $\begin{array}{l}-4.51 \\
-5.73 \\
-4.96 \\
-2.00 \\
-4.88\end{array}$ & & $\begin{array}{l}.4751 \\
.4286 \\
.4495 \\
.5320 \\
.4450\end{array}$ & $\begin{array}{l}.4686 \\
.4207 \\
.4423 \\
.5347 \\
.4393\end{array}$ & $\begin{array}{r}2.7 \\
-4.9 \\
3.8 \\
-.2 \\
-8.3\end{array}$ & $\begin{array}{r}7.1 \\
-3.9 \\
8.4 \\
2.6 \\
-8.5\end{array}$ & $\begin{array}{r}-.007 \\
-.009 \\
.020 \\
-.012 \\
-.029\end{array}$ \\
\hline $\begin{array}{r}95 \\
96 \\
117 \\
118 \\
119\end{array}$ & $\begin{array}{r}119.1 \\
80.2 \\
108.3 \\
120.4 \\
161.4\end{array}$ & $\begin{array}{l}13.88 \\
15.58 \\
10.92 \\
12.28 \\
14.08\end{array}$ & $\begin{array}{l}14.08 \\
15.69 \\
10.85 \\
12.37 \\
14.46\end{array}$ & $\begin{array}{l}-8.09 \\
-6.40 \\
11.82 \\
13.21 \\
14.57\end{array}$ & $\begin{array}{l}-8.14 \\
-6.31 \\
11.82 \\
13.21 \\
14.56\end{array}$ & & $\begin{array}{r}.3513 \\
.3972 \\
1.4410 \\
1.6185 \\
1.9305\end{array}$ & $\begin{array}{r}.3470 \\
.3982 \\
1.4408 \\
1.6189 \\
1.9301\end{array}$ & $\begin{array}{r}-2.7 \\
2.4 \\
1.2 \\
-.7 \\
1.1\end{array}$ & $\begin{array}{r}-1.3 \\
4.7 \\
4.6 \\
.8 \\
6.9\end{array}$ & $\begin{array}{r}.022 \\
.010 \\
.010 \\
-.006 \\
.014\end{array}$ \\
\hline $\begin{array}{l}120 \\
121 \\
122 \\
123 \\
124\end{array}$ & $\begin{array}{l}110.7 \\
146.8 \\
140.4 \\
132.9 \\
157.2\end{array}$ & $\begin{array}{l}17.17 \\
17.20 \\
19.22 \\
19.88 \\
23.10\end{array}$ & $\begin{array}{l}17.49 \\
17.58 \\
19.48 \\
20.31 \\
23.30\end{array}$ & $\begin{array}{l}17.51 \\
17.65 \\
19.50 \\
20.06 \\
22.73\end{array}$ & $\begin{array}{l}17.51 \\
17.65 \\
19.50 \\
20.06 \\
22.71\end{array}$ & & $\begin{array}{l}2.1671 \\
2.3218 \\
2.6015 \\
2.6460 \\
3.1029\end{array}$ & $\begin{array}{l}2.1673 \\
2.3218 \\
2.6012 \\
2.6462 \\
3.1014\end{array}$ & $\begin{array}{l}2.5 \\
4.9 \\
7.8 \\
5.8 \\
8.3\end{array}$ & $\begin{array}{r}7.5 \\
13.8 \\
16.7 \\
14.7 \\
17.9\end{array}$ & $\begin{array}{l}.001 \\
.012 \\
.012 \\
.003 \\
.018\end{array}$ \\
\hline
\end{tabular}




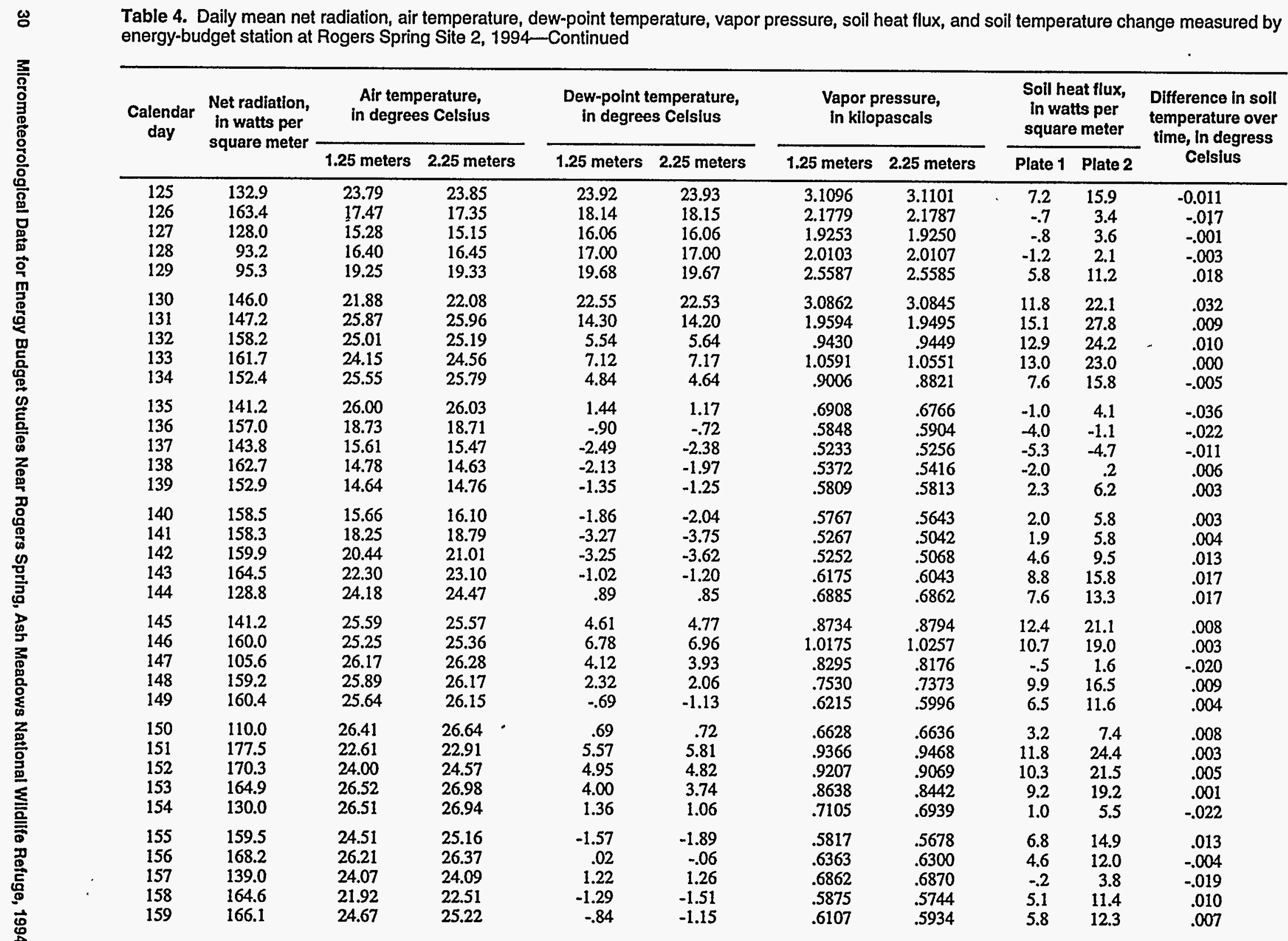


Table 4. Daily mean net radiation, air temperature, dew-point temperature, vapor pressure, soil heat flux, and soil temperature change measured by energy-budget station at Rogers Spring Site 2, 1994-Continued

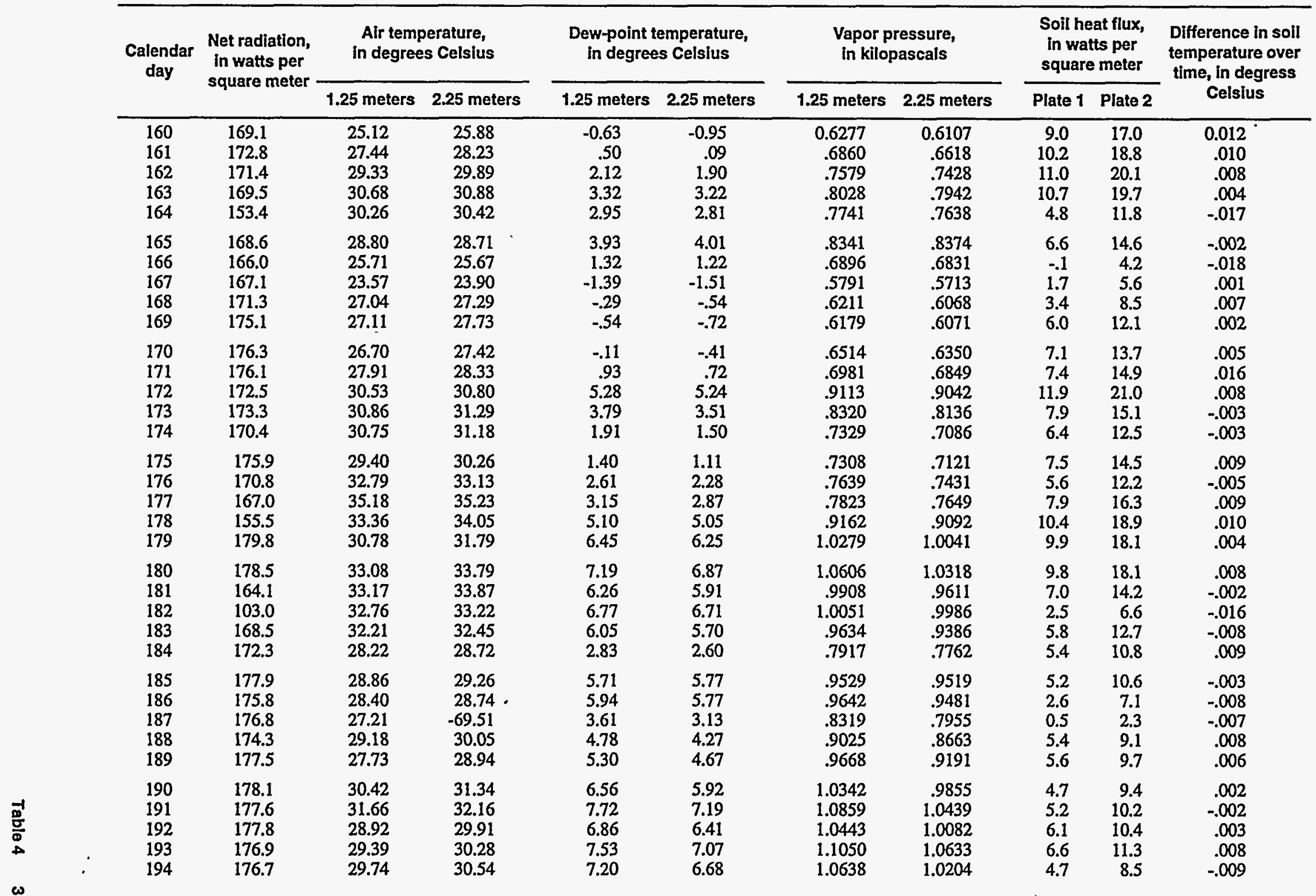


w Table 4. Daily mean net radiation, air temperature, dew-point temperature, vapor pressure, soil heat flux, and soil temperature change measured by energy-budget station at Rogers Spring Site 2, 1994-Continued

\begin{tabular}{|c|c|c|c|c|c|c|c|c|c|c|}
\hline \multirow[t]{2}{*}{$\begin{array}{l}\text { Calendar } \\
\text { day }\end{array}$} & \multirow{2}{*}{$\begin{array}{l}\text { Net radiation, } \\
\text { in watts per } \\
\text { square meter }\end{array}$} & \multicolumn{2}{|c|}{$\begin{array}{l}\text { Air temperature, } \\
\text { in degrees Celsius }\end{array}$} & \multicolumn{2}{|c|}{$\begin{array}{l}\text { Dew-point temperature, } \\
\text { in degrees Celsius }\end{array}$} & \multicolumn{2}{|c|}{$\begin{array}{l}\text { Vapor pressure, } \\
\text { in kilopascals }\end{array}$} & \multicolumn{2}{|c|}{$\begin{array}{l}\text { Soll heat flux, } \\
\text { in watts per } \\
\text { square meter }\end{array}$} & \multirow{2}{*}{$\begin{array}{c}\text { Difference in soil } \\
\text { temperature over } \\
\text { time, in degress } \\
\text { Celsius }\end{array}$} \\
\hline & & 1.25 meters & 2.25 meters & 1.25 meters & 2.25 meters & 1.25 meters & 2.25 meters & Plate 1 & Plate 2 & \\
\hline $\begin{array}{l}195 \\
196 \\
197 \\
198 \\
199\end{array}$ & $\begin{array}{l}170.5 \\
179.3 \\
165.0 \\
154.1 \\
165.3\end{array}$ & $\begin{array}{l}30.32 \\
30.47 \\
30.48 \\
31.30 \\
31.59\end{array}$ & $\begin{array}{l}31.10 \\
31.29 \\
31.16 \\
32.06 \\
31.77\end{array}$ & $\begin{array}{r}6.99 \\
7.41 \\
6.68 \\
9.15 \\
11.10\end{array}$ & $\begin{array}{r}6.54 \\
7.01 \\
6.15 \\
8.81 \\
10.90\end{array}$ & $\begin{array}{l}1.0537 \\
1.0785 \\
1.0102 \\
1.1885 \\
1.3409\end{array}$ & $\begin{array}{r}1.0172 \\
1.0448 \\
.9697 \\
1.1580 \\
1.3210\end{array}$ & $\begin{array}{l}3.9 \\
5.2 \\
4.3 \\
9.1 \\
7.8\end{array}$ & $\begin{array}{r}7.5 \\
9.0 \\
7.3 \\
15.1 \\
13.2\end{array}$ & $\begin{array}{r}0.001 \\
.000 \\
.003 \\
.016 \\
-.001\end{array}$ \\
\hline $\begin{array}{l}200 \\
201 \\
202 \\
203 \\
204\end{array}$ & $\begin{array}{l}165.9 \\
157.0 \\
169.5 \\
184.4 \\
188.2\end{array}$ & $\begin{array}{l}29.06 \\
28.32 \\
29.48 \\
30.96 \\
30.43\end{array}$ & $\begin{array}{l}29.34 \\
28.57 \\
30.08 \\
31.54 \\
31.23\end{array}$ & $\begin{array}{r}11.95 \\
13.36 \\
12.59 \\
11.11 \\
9.88\end{array}$ & $\begin{array}{r}11.93 \\
13.34 \\
12.47 \\
10.79 \\
9.57\end{array}$ & $\begin{array}{l}1.4250 \\
1.5702 \\
1.5052 \\
1.3559 \\
1.2609\end{array}$ & $\begin{array}{l}1.4188 \\
1.5608 \\
1.4881 \\
1.3230 \\
1.2296\end{array}$ & $\begin{array}{l}8.3 \\
7.7 \\
7.1 \\
8.2 \\
7.5\end{array}$ & $\begin{array}{l}13.0 \\
14.7 \\
13.9 \\
15.7 \\
14.2\end{array}$ & $\begin{array}{r}.000 \\
.003 \\
-.000 \\
.006 \\
.000\end{array}$ \\
\hline $\begin{array}{l}205 \\
206 \\
207 \\
208 \\
209\end{array}$ & $\begin{array}{l}180.0 \\
175.0 \\
168.8 \\
180.6 \\
177.9\end{array}$ & $\begin{array}{l}31.06 \\
30.27 \\
29.12 \\
30.71 \\
31.45\end{array}$ & $\begin{array}{l}31.74 \\
31.15 \\
30.42 \\
31.60 \\
32.59\end{array}$ & $\begin{array}{l}8.84 \\
6.08 \\
5.60 \\
6.43 \\
7.35\end{array}$ & $\begin{array}{l}8.47 \\
5.57 \\
5.18 \\
6.04 \\
7.11\end{array}$ & $\begin{array}{r}1.1807 \\
.9895 \\
.9641 \\
1.0174 \\
1.0703\end{array}$ & $\begin{array}{r}1.1481 \\
.9505 \\
.9317 \\
.9849 \\
1.0487\end{array}$ & $\begin{array}{l}6.8 \\
6.3 \\
5.7 \\
7.7 \\
8.6\end{array}$ & $\begin{array}{l}12.6 \\
11.7 \\
10.4 \\
13.7 \\
15.0\end{array}$ & $\begin{array}{r}-.007 \\
.007 \\
-.003 \\
.009 \\
.005\end{array}$ \\
\hline $\begin{array}{l}210 \\
211 \\
212 \\
213 \\
214\end{array}$ & $\begin{array}{l}156.6 \\
179.2 \\
177.9 \\
176.9 \\
173.6\end{array}$ & $\begin{array}{l}32.37 \\
32.64 \\
31.39 \\
32.03 \\
30.92\end{array}$ & $\begin{array}{l}32.98 \\
33.01 \\
31.90 \\
32.51 \\
31.51\end{array}$ & $\begin{array}{l}9.41 \\
9.75 \\
6.51 \\
5.26 \\
2.91\end{array}$ & $\begin{array}{l}9.41 \\
9.67 \\
6.31 \\
5.01 \\
2.56\end{array}$ & $\begin{array}{r}1.2155 \\
1.2269 \\
.9969 \\
.9128 \\
.7785\end{array}$ & $\begin{array}{r}1.2076 \\
1.2181 \\
.9793 \\
.8942 \\
.7562\end{array}$ & $\begin{array}{l}8.4 \\
9.9 \\
6.5 \\
7.6 \\
6.4\end{array}$ & $\begin{array}{l}14.8 \\
17.2 \\
11.5 \\
13.0 \\
10.7\end{array}$ & $\begin{array}{r}.010 \\
-.003 \\
-.008 \\
-.002 \\
.000\end{array}$ \\
\hline $\begin{array}{l}215 \\
216 \\
217 \\
218 \\
219\end{array}$ & $\begin{array}{l}177.4 \\
176.6 \\
171.4 \\
172.6 \\
165.1\end{array}$ & $\begin{array}{l}27.84 \\
27.33 \\
30.78 \\
33.29 \\
32.51\end{array}$ & $\begin{array}{l}29.02 \\
28.32 \\
31.63 \\
34.04 \\
33.32\end{array}$ & $\begin{array}{r}.45 \\
-.91 \\
.19 \\
4.25 \\
5.53\end{array}$ & $\begin{array}{r}.10 \\
-1.30 \\
.06 \\
4.19 \\
5.44\end{array}$ & $\begin{array}{l}.6775 \\
.6260 \\
.6602 \\
.8596 \\
.9391\end{array}$ & $\begin{array}{l}.6504 \\
.6052 \\
.6515 \\
.8524 \\
.9287\end{array}$ & $\begin{array}{r}3.7 \\
3.3 \\
-2.9 \\
9.0 \\
7.7\end{array}$ & $\begin{array}{r}6.6 \\
5.8 \\
-2.6 \\
7.6 \\
6.1\end{array}$ & $\begin{array}{l}-.014 \\
-.005 \\
.003 \\
.019 \\
.004\end{array}$ \\
\hline $\begin{array}{l}220 \\
221 \\
222 \\
223 \\
224\end{array}$ & $\begin{array}{l}117.3 \\
156.5 \\
154.4 \\
176.6 \\
160.1\end{array}$ & $\begin{array}{l}32.03 \\
31.33 \\
30.65 \\
31.81 \\
32.86\end{array}$ & $\begin{array}{l}32.23 \\
31.42 \\
31.21 \\
32.47 \\
33.14\end{array}$ & $\begin{array}{r}6.53 \\
10.66 \\
12.12 \\
11.82 \\
11.81\end{array}$ & $\begin{array}{r}6.73 \\
10.94 \\
12.27 \\
11.79 \\
11.83\end{array}$ & $\begin{array}{l}.9817 \\
1.3023 \\
1.4429 \\
1.4201 \\
1.4052\end{array}$ & $\begin{array}{r}.9960 \\
1.3242 \\
1.4525 \\
1.4116 \\
1.4042\end{array}$ & $\begin{array}{r}4.3 \\
14.4 \\
12.8 \\
14.8 \\
13.5 \\
\end{array}$ & $\begin{array}{r}3.7 \\
11.7 \\
9.9 \\
11.5 \\
10.5\end{array}$ & $\begin{array}{r}-.001 \\
.016 \\
-.005 \\
.008 \\
.009\end{array}$ \\
\hline $\begin{array}{l}225 \\
226 \\
227 \\
228 \\
229\end{array}$ & $\begin{array}{l}162.8 \\
176.3 \\
175.4 \\
170.0 \\
146.5\end{array}$ & $\begin{array}{l}31.32 \\
29.92 \\
30.25 \\
33.33 \\
30.66\end{array}$ & $\begin{array}{l}31.76 \\
30.92 \\
31.57 \\
33.93 \\
31.04\end{array}$ & $\begin{array}{r}12.55 \\
12.08 \\
9.99 \\
9.49 \\
9.76\end{array}$ & $\begin{array}{r}12.66 \\
12.07 \\
9.82 \\
9.31 \\
9.88\end{array}$ & $\begin{array}{l}1.4794 \\
1.4602 \\
1.2697 \\
1.2220 \\
1.2346\end{array}$ & $\begin{array}{l}1.4858 \\
1.4500 \\
1.2527 \\
1.2020 \\
1.2409\end{array}$ & $\begin{array}{r}14.4 \\
11.6 \\
10.7 \\
12.6 \\
8.1\end{array}$ & $\begin{array}{r}10.7 \\
8.6 \\
7.9 \\
9.8 \\
5.8\end{array}$ & $\begin{array}{r}-.004 \\
-.000 \\
-.002 \\
.014 \\
-.014\end{array}$ \\
\hline
\end{tabular}


Table 4. Daily mean net radiation, air temperature, dew-point temperature, vapor pressure, soil heat flux, and soil temperature change measured by energy-budget station at Rogers Spring Site 2, 1994-Continued

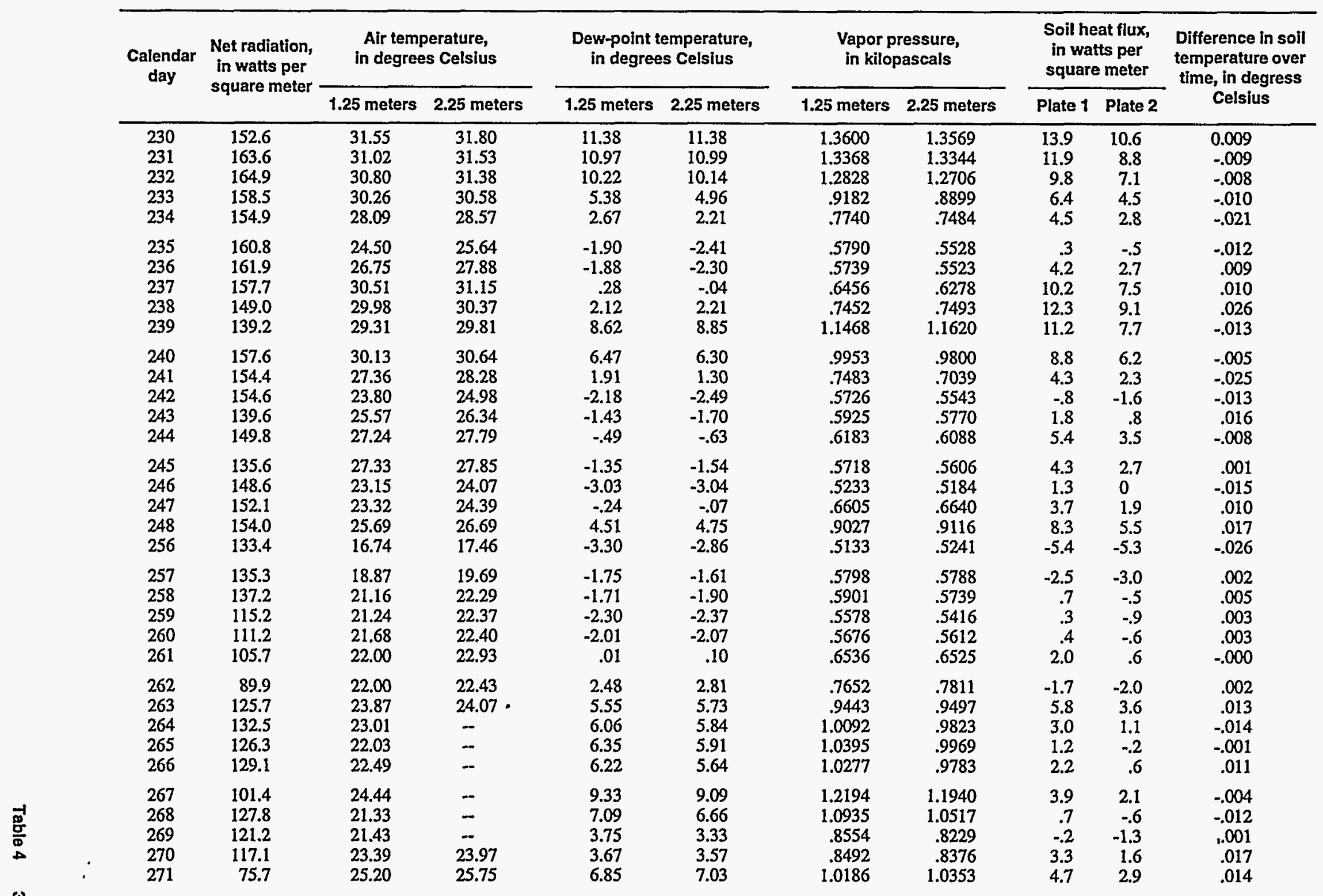


Table 4. Daily mean net radiation, air temperature, dew-point temperature, vapor pressure, soil heat flux, and soil temperature change measured by energy-budget station at Rogers Spring Site 2, 1994-Continued

\begin{tabular}{|c|c|c|c|c|c|c|c|c|c|c|}
\hline \multirow[t]{2}{*}{$\begin{array}{l}\text { Calendar } \\
\text { day }\end{array}$} & \multirow{2}{*}{$\begin{array}{l}\text { Net radiation, } \\
\text { in watts per } \\
\text { square meter }\end{array}$} & \multicolumn{2}{|c|}{$\begin{array}{l}\text { Air temperature, } \\
\text { in degrees Celsius }\end{array}$} & \multicolumn{2}{|c|}{$\begin{array}{l}\text { Dew-point temperature, } \\
\text { in degrees Celsius }\end{array}$} & \multicolumn{2}{|c|}{$\begin{array}{l}\text { Vapor pressure, } \\
\text { in kilopascals }\end{array}$} & \multicolumn{2}{|c|}{$\begin{array}{l}\text { Soil heat flux, } \\
\text { in watts per } \\
\text { square meter }\end{array}$} & \multirow{2}{*}{$\begin{array}{c}\text { Difference in soil } \\
\text { temperature over } \\
\text { time, in degress } \\
\text { Celsius }\end{array}$} \\
\hline & & 1.25 meters & 2.25 meters & 1.25 meters & 2.25 meters & 1.25 meters & 2.25 meters & Plate 1 & Plate 2 & \\
\hline $\begin{array}{l}272 \\
273 \\
274 \\
275 \\
276\end{array}$ & $\begin{array}{r}69.2 \\
92.7 \\
86.7 \\
76.3 \\
4.6\end{array}$ & $\begin{array}{l}22.59 \\
18.77 \\
18.47 \\
21.38 \\
19.08\end{array}$ & $\begin{array}{l}22.90 \\
19.35 \\
19.37 \\
21.92 \\
19.68\end{array}$ & $\begin{array}{r}6.91 \\
2.57 \\
2.23 \\
3.60 \\
.14\end{array}$ & $\begin{array}{r}7.19 \\
2.58 \\
2.23 \\
3.44 \\
.25\end{array}$ & $\begin{array}{l}1.0263 \\
.7743 \\
.7796 \\
.8395 \\
.6251\end{array}$ & $\begin{array}{r}1.0440 \\
.7688 \\
.7711 \\
.8227 \\
.6266\end{array}$ & $\begin{array}{r}-1.4 \\
-3.2 \\
-2.3 \\
1.0 \\
-6.6\end{array}$ & $\begin{array}{r}-1.8 \\
-3.5 \\
-2.8 \\
-.2 \\
-5.8\end{array}$ & $\begin{array}{r}-0.039 \\
-.015 \\
.010 \\
.011 \\
-.021\end{array}$ \\
\hline $\begin{array}{l}277 \\
278 \\
279 \\
280 \\
281\end{array}$ & $\begin{array}{l}47.6 \\
70.7 \\
79.9 \\
69.7 \\
63.8\end{array}$ & $\begin{array}{l}18.81 \\
14.75 \\
14.57 \\
18.22 \\
19.21\end{array}$ & $\begin{array}{l}18.82 \\
15.22 \\
15.09 \\
18.77 \\
19.88\end{array}$ & $\begin{array}{l}2.60 \\
1.52 \\
-.17 \\
-.86 \\
-.20\end{array}$ & $\begin{array}{l}2.90 \\
1.81 \\
-.06 \\
-.75 \\
-.34\end{array}$ & $\begin{array}{l}.7427 \\
.7076 \\
.6600 \\
.6107 \\
.6379\end{array}$ & $\begin{array}{l}.7578 \\
.7184 \\
.6551 \\
.6058 \\
.6266\end{array}$ & $\begin{array}{r}-1.9 \\
-6.1 \\
-6.9 \\
-3.5 \\
-.5\end{array}$ & $\begin{array}{l}-2.0 \\
-5.5 \\
-6.3 \\
-3.5 \\
-1.3\end{array}$ & $\begin{array}{l}.001 \\
.037 \\
.006 \\
.011 \\
.008\end{array}$ \\
\hline $\begin{array}{l}282 \\
283 \\
284 \\
285 \\
286\end{array}$ & $\begin{array}{l}72.5 \\
70.7 \\
62.3 \\
60.4 \\
57.2\end{array}$ & $\begin{array}{l}16.28 \\
17.16 \\
18.65 \\
19.66 \\
15.70\end{array}$ & $\begin{array}{l}17.17 \\
18.19 \\
19.56 \\
20.06 \\
16.16\end{array}$ & $\begin{array}{l}-2.75 \\
-2.93 \\
-1.43 \\
-1.13 \\
-1.68\end{array}$ & $\begin{array}{l}-2.87 \\
-3.00 \\
-1.51 \\
-1.17 \\
-1.68\end{array}$ & $\begin{array}{l}.5578 \\
.5420 \\
.5868 \\
.5965 \\
.5731\end{array}$ & $\begin{array}{l}.5449 \\
.5334 \\
.5788 \\
.5913 \\
.5687\end{array}$ & $\begin{array}{r}-5.1 \\
-4.4 \\
-1.9 \\
-.1 \\
-6.0\end{array}$ & $\begin{array}{r}-5.0 \\
-4.3 \\
-2.4 \\
-.7 \\
-5.5\end{array}$ & $\begin{array}{r}-0.003 \\
-.002 \\
-.004 \\
.029 \\
.020\end{array}$ \\
\hline $\begin{array}{l}287 \\
288 \\
289 \\
290 \\
291\end{array}$ & $\begin{array}{l}40.2 \\
53.1 \\
55.6 \\
61.1 \\
61.9\end{array}$ & $\begin{array}{l}17.07 \\
13.16 \\
11.52 \\
10.50 \\
11.48\end{array}$ & $\begin{array}{l}17.20 \\
13.21 \\
11.66 \\
11.03 \\
12.14\end{array}$ & $\begin{array}{r}.83 \\
-2.49 \\
-4.41 \\
-3.45 \\
-6.73\end{array}$ & $\begin{array}{r}1.11 \\
-2.45 \\
-4.39 \\
-3.41 \\
-6.78\end{array}$ & $\begin{array}{l}.6633 \\
.5220 \\
.4611 \\
.5120 \\
.3841\end{array}$ & $\begin{array}{l}.6744 \\
.5226 \\
.4585 \\
.5090 \\
.3832\end{array}$ & $\begin{array}{r}-2.4 \\
-9.3 \\
-10.9 \\
-10.7 \\
-9.7\end{array}$ & $\begin{array}{l}-2.5 \\
-7.9 \\
-9.1 \\
-9.2 \\
-8.4\end{array}$ & $\begin{array}{r}.002 \\
-.037 \\
.007 \\
-.018 \\
.000\end{array}$ \\
\hline $\begin{array}{l}292 \\
293 \\
294 \\
295 \\
296\end{array}$ & $\begin{array}{l}59.1 \\
56.8 \\
57.0 \\
41.4 \\
53.3\end{array}$ & $\begin{array}{l}11.90 \\
12.17 \\
12.17 \\
12.39 \\
14.27\end{array}$ & $\begin{array}{l}12.72 \\
12.92 \\
13.04 \\
13.26 \\
14.92\end{array}$ & $\begin{array}{l}-7.52 \\
-3.80 \\
-7.41 \\
-8.36 \\
-8.22\end{array}$ & $\begin{array}{l}-7.17 \\
-3.92 \\
-7.29 \\
-8.23 \\
-8.22\end{array}$ & $\begin{array}{l}.3651 \\
.4977 \\
.3732 \\
.3438 \\
.3471\end{array}$ & $\begin{array}{l}.3734 \\
.4892 \\
.3748 \\
.3460 \\
.3460\end{array}$ & $\begin{array}{l}-8.9 \\
-8.1 \\
-7.6 \\
-7.8 \\
-4.4\end{array}$ & $\begin{array}{l}-7.9 \\
-7.4 \\
-7.3 \\
-7.2 \\
-4.2\end{array}$ & $\begin{array}{r}.001 \\
.000 \\
.000 \\
.002 \\
.021\end{array}$ \\
\hline $\begin{array}{l}297 \\
298 \\
299 \\
300 \\
301\end{array}$ & $\begin{array}{l}56.8 \\
54.3 \\
50.4 \\
46.5 \\
48.4\end{array}$ & $\begin{array}{l}15.77 \\
14.57 \\
14.38 \\
14.47 \\
13.20\end{array}$ & $\begin{array}{l}16.52 \\
15.42 \\
15.15 \\
15.35 \\
14.15\end{array}$ & $\begin{array}{l}-7.96 \\
-2.75 \\
-2.43 \\
-3.14 \\
-3.66\end{array}$ & $\begin{array}{l}-7.82 \\
-2.80 \\
-2.45 \\
-3.11 \\
-3.67\end{array}$ & $\begin{array}{l}.3437 \\
.5739 \\
.5598 \\
.5265 \\
.5083\end{array}$ & $\begin{array}{l}.3469 \\
.5670 \\
.5527 \\
.5231 \\
.5026\end{array}$ & $\begin{array}{l}-1.8 \\
-5.3 \\
-6.1 \\
-6.6 \\
-8.2\end{array}$ & $\begin{array}{l}-1.9 \\
-4.6 \\
-5.2 \\
-5.6 \\
-6.8\end{array}$ & $\begin{array}{l}-.004 \\
-.006 \\
-.003 \\
-.004 \\
-.009\end{array}$ \\
\hline $\begin{array}{l}302 \\
303 \\
304 \\
305 \\
306\end{array}$ & $\begin{array}{l}43.6 \\
33.5 \\
44.1 \\
29.1 \\
11.6\end{array}$ & $\begin{array}{l}14.18 \\
13.50 \\
10.72 \\
15.31 \\
14.13\end{array}$ & $\begin{array}{l}14.84 \\
14.30 \\
11.63 \\
15.52 \\
14.36\end{array}$ & $\begin{array}{l}-3.92 \\
-5.88 \\
-9.54 \\
-7.63 \\
-4.03\end{array}$ & $\begin{array}{l}-3.95 \\
-6.18 \\
-9.97 \\
-7.83 \\
-3.71\end{array}$ & $\begin{array}{l}.4981 \\
.4207 \\
.3277 \\
.3772 \\
.4580\end{array}$ & $\begin{array}{l}.4929 \\
.4094 \\
.3131 \\
.3700 \\
.4688\end{array}$ & $\begin{array}{r}-8.6 \\
-10.8 \\
-14.2 \\
-7.8 \\
-7.3\end{array}$ & $\begin{array}{r}-6.9 \\
-8.8 \\
-11.7 \\
-6.0 \\
-6.0\end{array}$ & $\begin{array}{r}.001 \\
. .025 \\
-.005 \\
.031 \\
. .019\end{array}$ \\
\hline
\end{tabular}


Table 4. Daily mean net radiation, air temperature, dew-point temperature, vapor pressure, soil heat flux, and soil temperature change measured by energy-budget station at Rogers Spring Site 2, 1994-Continued

\begin{tabular}{|c|c|c|c|c|c|c|c|c|c|c|}
\hline \multirow{2}{*}{$\begin{array}{l}\text { Calendar } \\
\text { day }\end{array}$} & \multirow{2}{*}{$\begin{array}{l}\text { Net radiation, } \\
\text { in watts per } \\
\text { square meter }\end{array}$} & \multicolumn{2}{|c|}{$\begin{array}{l}\text { Air temperature, } \\
\text { in degrees Celsius }\end{array}$} & \multicolumn{2}{|c|}{$\begin{array}{l}\text { Dew-point temperature, } \\
\text { in degrees Celsius }\end{array}$} & \multicolumn{2}{|c|}{$\begin{array}{l}\text { Vapor pressure, } \\
\text { in kilopascals }\end{array}$} & \multicolumn{2}{|c|}{$\begin{array}{l}\text { Soil heat flux, } \\
\text { in watts per } \\
\text { square meter }\end{array}$} & \multirow{2}{*}{$\begin{array}{c}\text { Difference in soil } \\
\text { temperature over } \\
\text { time, in degress } \\
\text { Celslus }\end{array}$} \\
\hline & & 1.25 meters & 2.25 meters & 1.25 meters & 2.25 meters & 1.25 meters & 2.25 meters & Plate 1 & Plate 2 & \\
\hline $\begin{array}{l}307 \\
308 \\
309 \\
310 \\
311\end{array}$ & $\begin{array}{l}25.0 \\
34.0 \\
46.9 \\
37.8 \\
13.7\end{array}$ & $\begin{array}{r}7.36 \\
4.60 \\
8.26 \\
12.31 \\
10.32\end{array}$ & $\begin{array}{r}7.37 \\
5.10 \\
8.65 \\
12.77 \\
10.96\end{array}$ & $\begin{array}{r}-7.37 \\
-11.10 \\
-9.85 \\
-7.64 \\
-8.83\end{array}$ & $\begin{array}{r}-7.24 \\
-11.19 \\
-9.96 \\
-7.60 \\
-8.82\end{array}$ & $\begin{array}{r}0.3594 \\
.2766 \\
.3050 \\
.3628 \\
.3248\end{array}$ & $\begin{array}{r}0.3628 \\
.2729 \\
.3011 \\
.3618 \\
.3242\end{array}$ & $\begin{array}{r}-19.8 \\
-21.3 \\
-13.6 \\
-8.8 \\
-11.7\end{array}$ & $\begin{array}{r}-16.4 \\
-17.7 \\
-11.3 \\
-7.2 \\
-9.8\end{array}$ & $\begin{array}{r}-0.038 \\
-.008 \\
.008 \\
.014 \\
-.015\end{array}$ \\
\hline $\begin{array}{l}312 \\
313 \\
314 \\
315 \\
316\end{array}$ & $\begin{array}{r}45.5 \\
34.1 \\
6.5 \\
35.5 \\
49.7\end{array}$ & $\begin{array}{r}10.29 \\
12.64 \\
10.03 \\
5.44 \\
5.07\end{array}$ & $\begin{array}{r}10.88 \\
12.88 \\
10.18 \\
5.62 \\
5.38\end{array}$ & $\begin{array}{l}-4.86 \\
-2.01 \\
-1.95 \\
-4.06 \\
-4.24\end{array}$ & $\begin{array}{l}-4.64 \\
-1.80 \\
-1.52 \\
-3.72 \\
-4.03\end{array}$ & $\begin{array}{l}.4601 \\
.5516 \\
.5360 \\
.4745 \\
.4766\end{array}$ & $\begin{array}{l}.4635 \\
.5571 \\
.5523 \\
.4841 \\
.4819\end{array}$ & $\begin{array}{r}-8.2 \\
-6.9 \\
-9.4 \\
-15.2 \\
-16.3\end{array}$ & $\begin{array}{r}-6.9 \\
-5.6 \\
-7.8 \\
-12.8 \\
-13.6\end{array}$ & $\begin{array}{r}.012 \\
.014 \\
-.022 \\
-.003 \\
-.005\end{array}$ \\
\hline $\begin{array}{l}317 \\
318 \\
319 \\
320\end{array}$ & $\begin{array}{l}15.4 \\
19.0 \\
32.5 \\
30.3\end{array}$ & $\begin{array}{l}7.45 \\
5.17 \\
5.23 \\
9.31\end{array}$ & $\begin{array}{l}7.50 \\
5.50 \\
5.53 \\
9.35\end{array}$ & $\begin{array}{r}-4.60 \\
-7.70 \\
2.58 \\
10.24\end{array}$ & $\begin{array}{r}-4.87 \\
-7.94 \\
2.69 \\
10.26\end{array}$ & $\begin{array}{r}.4452 \\
.3603 \\
1.0106 \\
1.3057\end{array}$ & $\begin{array}{r}.4346 \\
.3511 \\
1.0118 \\
1.3067\end{array}$ & $\begin{array}{r}-15.7 \\
-18.1 \\
-16.2 \\
-6.1\end{array}$ & $\begin{array}{r}-12.9 \\
-14.8 \\
-13.3 \\
-5.0\end{array}$ & $\begin{array}{r}-.013 \\
-.019 \\
.021 \\
-.007\end{array}$ \\
\hline
\end{tabular}


Table 5. Daily mean, maximum, and minimum air and dew-point temperatures and vapor pressure measured by energy-budget station at Rogers Spring Site 2, 1994

$[-$, no data available $]$

\begin{tabular}{|c|c|c|c|c|c|c|c|c|c|}
\hline \multirow{2}{*}{$\begin{array}{c}\text { Calendar } \\
\text { day }\end{array}$} & \multicolumn{3}{|c|}{$\begin{array}{c}\text { Air temperature at } 1.25 \text { meters, } \\
\text { in degrees Celsius }\end{array}$} & \multicolumn{3}{|c|}{$\begin{array}{l}\text { Dew-point temperature at } 1.25 \\
\text { meters, in degrees Celsius }\end{array}$} & \multicolumn{3}{|c|}{$\begin{array}{l}\text { Vapor pressure at } 1.25 \text { moters, } \\
\text { in kilopascals }\end{array}$} \\
\hline & Mean & Maximum & Minimum & Mean & Maximum & Minimum & Mean & Maximum & Minimum \\
\hline $\begin{array}{l}75 \\
76 \\
77 \\
78 \\
79\end{array}$ & $\begin{array}{l}22.32 \\
19.12 \\
19.47 \\
15.69 \\
13.23\end{array}$ & $\begin{array}{l}27.43 \\
25.55 \\
24.32 \\
19.87 \\
21.26\end{array}$ & $\begin{array}{r}18.57 \\
11.18 \\
14.95 \\
9.77 \\
6.44\end{array}$ & $\begin{array}{l}-6.19 \\
-6.14 \\
-3.16 \\
-2.41 \\
-3.36\end{array}$ & $\begin{array}{r}-3.95 \\
-1.21 \\
-.30 \\
-.63 \\
-1.36\end{array}$ & $\begin{array}{r}-9.27 \\
-10.60 \\
-8.88 \\
-4.53 \\
-7.06\end{array}$ & $\begin{array}{r}0.3877 \\
.3973 \\
.4910 \\
.5161 \\
.4822\end{array}$ & $\begin{array}{r}0.4563 \\
.5593 \\
.5978 \\
.5836 \\
.5530\end{array}$ & $\begin{array}{r}0.3034 \\
.2731 \\
.3128 \\
.4460 \\
.3603\end{array}$ \\
\hline $\begin{array}{l}80 \\
81 \\
82 \\
83 \\
84\end{array}$ & $\begin{array}{r}15.28 \\
13.35 \\
10.28 \\
12.89 \\
9.74\end{array}$ & $\begin{array}{l}22.90 \\
17.90 \\
17.27 \\
18.72 \\
15.84\end{array}$ & $\begin{array}{r}3.64 \\
2.29 \\
.19 \\
8.83 \\
5.14\end{array}$ & $\begin{array}{l}-.33 \\
-3.14 \\
-8.12 \\
-4.89 \\
-3.91\end{array}$ & $\begin{array}{r}7.99 \\
2.84 \\
-2.34 \\
.01 \\
.93\end{array}$ & $\begin{array}{r}-8.97 \\
-14.03 \\
-14.93 \\
-7.76 \\
-7.08\end{array}$ & $\begin{array}{l}.6495 \\
.5147 \\
.3485 \\
.4336 \\
.4671\end{array}$ & $\begin{array}{r}1.0714 \\
.7488 \\
.5149 \\
.6117 \\
.6536\end{array}$ & $\begin{array}{l}.3106 \\
.2071 \\
.1924 \\
.3413 \\
.3599\end{array}$ \\
\hline $\begin{array}{l}85 \\
86 \\
87 \\
88 \\
89\end{array}$ & $\begin{array}{l}12.36 \\
15.12 \\
15.77 \\
16.19 \\
18.34\end{array}$ & $\begin{array}{l}21.87 \\
25.37 \\
25.62 \\
26.82 \\
27.84\end{array}$ & $\begin{array}{r}.61 \\
4.81 \\
1.78 \\
3.98 \\
8.29\end{array}$ & $\begin{array}{l}-2.59 \\
-4.67 \\
-5.34 \\
-5.31 \\
-3.97\end{array}$ & $\begin{array}{l}4.41 \\
2.34 \\
2.22 \\
2.40 \\
2.71\end{array}$ & $\begin{array}{r}-9.04 \\
-12.64 \\
-13.38 \\
-13.28 \\
-9.63\end{array}$ & $\begin{array}{l}.5351 \\
.4635 \\
.4425 \\
.4452 \\
.4814\end{array}$ & $\begin{array}{l}.8369 \\
.7226 \\
.7167 \\
.7261 \\
.7421\end{array}$ & $\begin{array}{l}.3090 \\
.2320 \\
.2185 \\
.2203 \\
.2950\end{array}$ \\
\hline $\begin{array}{l}90 \\
91 \\
92 \\
93 \\
94\end{array}$ & $\begin{array}{l}17.67 \\
16.28 \\
18.02 \\
19.81 \\
15.71\end{array}$ & $\begin{array}{l}28.25 \\
26.70 \\
26.80 \\
25.62 \\
23.82\end{array}$ & $\begin{array}{r}4.85 \\
2.20 \\
6.31 \\
12.00 \\
5.22\end{array}$ & $\begin{array}{l}-4.42 \\
-5.61 \\
-4.87 \\
-2.10 \\
-4.78\end{array}$ & $\begin{array}{r}3.09 \\
.89 \\
1.52 \\
1.89 \\
1.23\end{array}$ & $\begin{array}{r}-11.25 \\
-12.62 \\
-11.54 \\
-5.65 \\
-10.63\end{array}$ & $\begin{array}{l}.4751 \\
.4286 \\
.4495 \\
.5320 \\
.4450\end{array}$ & $\begin{array}{l}.7621 \\
.6514 \\
.6815 \\
.7001 \\
.6674\end{array}$ & $\begin{array}{l}.2595 \\
.2324 \\
.2535 \\
.4012 \\
.2725\end{array}$ \\
\hline $\begin{array}{r}95 \\
96 \\
117 \\
118 \\
119\end{array}$ & $\begin{array}{l}13.88 \\
15.58 \\
10.92 \\
12.28 \\
14.08\end{array}$ & $\begin{array}{l}22.37 \\
23.62 \\
16.82 \\
18.29 \\
23.51\end{array}$ & $\begin{array}{l}3.72 \\
5.06 \\
6.23 \\
6.75 \\
3.09\end{array}$ & $\begin{array}{l}-8.09 \\
-6.40 \\
11.82 \\
13.21 \\
14.57\end{array}$ & $\begin{array}{r}-1.92 \\
-.76 \\
20.77 \\
22.53 \\
27.72\end{array}$ & $\begin{array}{r}-15.15 \\
-13.07 \\
6.40 \\
6.37 \\
1.64\end{array}$ & $\begin{array}{r}.3513 \\
.3972 \\
1.4410 \\
1.6185 \\
1.9305\end{array}$ & $\begin{array}{r}.5308 \\
.5781 \\
2.4520 \\
2.7305 \\
3.7183\end{array}$ & $\begin{array}{l}.1889 \\
.2240 \\
.9605 \\
.9586 \\
.6878\end{array}$ \\
\hline $\begin{array}{l}120 \\
121 \\
122 \\
123 \\
124\end{array}$ & $\begin{array}{l}17.17 \\
17.20 \\
19.22 \\
19.88 \\
23.10\end{array}$ & $\begin{array}{l}24.06 \\
26.72 \\
28.48 \\
28.88 \\
31.31\end{array}$ & $\begin{array}{r}9.45 \\
8.13 \\
7.25 \\
8.98 \\
11.34\end{array}$ & $\begin{array}{l}17.51 \\
17.65 \\
19.50 \\
20.06 \\
22.73\end{array}$ & $\begin{array}{l}27.35 \\
30.91 \\
31.83 \\
32.22 \\
33.74\end{array}$ & $\begin{array}{l}8.92 \\
6.16 \\
7.05 \\
7.28 \\
9.77\end{array}$ & $\begin{array}{l}2.1671 \\
2.3218 \\
2.6015 \\
2.6460 \\
3.1029\end{array}$ & $\begin{array}{l}3.6382 \\
4.4692 \\
4.7092 \\
4.8156 \\
5.2447\end{array}$ & $\begin{array}{r}1.1408 \\
.9449 \\
1.0054 \\
1.0215 \\
1.2081\end{array}$ \\
\hline $\begin{array}{l}125 \\
126 \\
127 \\
128 \\
129\end{array}$ & $\begin{array}{l}23.79 \\
17.47 \\
15.28 \\
16.40 \\
19.25\end{array}$ & $\begin{array}{l}29.98 \\
22.92 \\
20.59 \\
21.78 \\
28.57\end{array}$ & $\begin{array}{r}18.08 \\
12.41 \\
8.63 \\
10.65 \\
8.64\end{array}$ & $\begin{array}{l}23.92 \\
18.14 \\
16.06 \\
17.00 \\
19.68\end{array}$ & $\begin{array}{l}32.54 \\
26.24 \\
24.98 \\
25.18 \\
32.79\end{array}$ & $\begin{array}{r}17.83 \\
11.10 \\
7.49 \\
10.21 \\
7.20\end{array}$ & $\begin{array}{l}3.1096 \\
2.1779 \\
1.9253 \\
2.0103 \\
2.5587\end{array}$ & $\begin{array}{l}4.9019 \\
3.4082 \\
3.1624 \\
3.2012 \\
4.9713\end{array}$ & $\begin{array}{l}2.0419 \\
1.3208 \\
1.0353 \\
1.2452 \\
1.0151\end{array}$ \\
\hline $\begin{array}{l}130 \\
131 \\
132 \\
133 \\
134\end{array}$ & $\begin{array}{l}21.88 \\
25.87 \\
25.01 \\
24.15 \\
25.55\end{array}$ & $\begin{array}{l}31.06 \\
34.45 \\
32.86 \\
33.63 \\
34.49\end{array}$ & $\begin{array}{r}8.72 \\
15.74 \\
12.08 \\
12.58 \\
10.93\end{array}$ & $\begin{array}{r}22.55 \\
14.30 \\
5.54 \\
7.12 \\
4.84\end{array}$ & $\begin{array}{l}34.90 \\
33.86 \\
10.87 \\
13.36 \\
10.70\end{array}$ & $\begin{array}{r}7.50 \\
1.58 \\
-2.42 \\
.69 \\
-2.87\end{array}$ & $\begin{array}{r}3.0862 \\
1.9594 \\
.9430 \\
1.0591 \\
.9006\end{array}$ & $\begin{array}{l}5.5931 \\
5.2814 \\
1.3001 \\
1.5320 \\
1.2855\end{array}$ & $\begin{array}{r}1.0359 \\
.6848 \\
.5114 \\
.6423 \\
.4947\end{array}$ \\
\hline $\begin{array}{l}135 \\
136 \\
137 \\
138 \\
139\end{array}$ & $\begin{array}{l}26.00 \\
18.73 \\
15.61 \\
14.78 \\
14.64\end{array}$ & $\begin{array}{l}31.36 \\
24.40 \\
21.48 \\
20.86 \\
22.89\end{array}$ & $\begin{array}{r}20.76 \\
9.56 \\
10.16 \\
8.55 \\
7.27\end{array}$ & $\begin{array}{r}1.44 \\
-.90 \\
-2.49 \\
-2.13 \\
-1.35\end{array}$ & $\begin{array}{l}5.41 \\
3.06 \\
2.19 \\
2.81 \\
4.72\end{array}$ & $\begin{array}{l}-3.33 \\
-7.28 \\
-7.17 \\
-7.10 \\
-7.48\end{array}$ & $\begin{array}{l}.6908 \\
.5848 \\
.5233 \\
.5372 \\
.5809\end{array}$ & $\begin{array}{l}.8969 \\
.7607 \\
.7153 \\
.7474 \\
.8552\end{array}$ & $\begin{array}{l}.4779 \\
.3541 \\
.3573 \\
.3592 \\
.3487\end{array}$ \\
\hline $\begin{array}{l}140 \\
141 \\
142 \\
143 \\
144\end{array}$ & $\begin{array}{l}15.66 \\
18.25 \\
20.44 \\
22.30 \\
24.18\end{array}$ & $\begin{array}{l}25.95 \\
29.78 \\
31.61 \\
33.55 \\
33.89\end{array}$ & $\begin{array}{r}4.23 \\
4.33 \\
6.65 \\
9.47 \\
10.66\end{array}$ & $\begin{array}{r}-1.86 \\
-3.27 \\
-3.25 \\
-1.02 \\
.89\end{array}$ & $\begin{array}{l}5.65 \\
4.65 \\
4.26 \\
6.29 \\
6.55\end{array}$ & $\begin{array}{r}-9.55 \\
-12.03 \\
-13.11 \\
-10.30 \\
-7.09\end{array}$ & $\begin{array}{l}.5767 \\
.5267 \\
.5252 \\
.6175 \\
.6885\end{array}$ & $\begin{array}{l}.9120 \\
.8508 \\
.8278 \\
.9536 \\
.9709\end{array}$ & $\begin{array}{l}.2968 \\
.2436 \\
.2248 \\
.2796 \\
.3594\end{array}$ \\
\hline
\end{tabular}


Table 5. Daily mean, maximum, and minimum air and dew-point temperatures and vapor pressure, measured by energy-budget station at Rogers Spring Site 2, 1994-Continued

\begin{tabular}{|c|c|c|c|c|c|c|c|c|c|}
\hline \multirow{2}{*}{$\begin{array}{c}\text { Calendar } \\
\text { day }\end{array}$} & \multicolumn{3}{|c|}{$\begin{array}{c}\text { Air temperature at } 1.25 \text { meters, } \\
\text { In degrees Celsius }\end{array}$} & \multicolumn{3}{|c|}{$\begin{array}{l}\text { Dew-point temperature at } 1.25 \\
\text { meters, in degrees Celsius }\end{array}$} & \multicolumn{3}{|c|}{$\begin{array}{c}\text { Vapor pressure at } 1.25 \text { moters, } \\
\text { in kllopascals }\end{array}$} \\
\hline & Mean & Maximum & Minimum & Mean & Maximum & Minimum & Mean & Maximum & Minimum \\
\hline $\begin{array}{l}145 \\
146 \\
147 \\
148 \\
149\end{array}$ & $\begin{array}{l}25.59 \\
25.25 \\
26.17 \\
25.89 \\
25.64\end{array}$ & $\begin{array}{l}32.89 \\
32.11 \\
31.10 \\
33.75 \\
35.57\end{array}$ & $\begin{array}{l}16.95 \\
13.95 \\
19.93 \\
17.10 \\
10.76\end{array}$ & $\begin{array}{l}4.61 \\
6.78 \\
4.12 \\
2.32 \\
-.69\end{array}$ & $\begin{array}{r}9.68 \\
11.84 \\
7.51 \\
8.10 \\
6.31\end{array}$ & $\begin{array}{r}-1.00 \\
-.20 \\
.54 \\
-4.57 \\
-9.72\end{array}$ & $\begin{array}{r}0.8734 \\
1.0175 \\
.8295 \\
.7530 \\
.6215\end{array}$ & $\begin{array}{r}1.2010 \\
1.3863 \\
1.0371 \\
1.0793 \\
.9548\end{array}$ & $\begin{array}{r}0.5680 \\
.6022 \\
.6352 \\
.4360 \\
.2927\end{array}$ \\
\hline $\begin{array}{l}150 \\
151 \\
152 \\
153 \\
154\end{array}$ & $\begin{array}{l}26.41 \\
22.61 \\
24.00 \\
26.52 \\
26.51\end{array}$ & $\begin{array}{l}35.19 \\
31.33 \\
34.79 \\
36.64 \\
34.62\end{array}$ & $\begin{array}{l}15.14 \\
15.01 \\
11.90 \\
12.10 \\
12.89\end{array}$ & $\begin{array}{r}.69 \\
5.57 \\
4.95 \\
4.00 \\
1.36\end{array}$ & $\begin{array}{r}5.19 \\
10.67 \\
11.53 \\
10.88 \\
7.74\end{array}$ & $\begin{array}{r}-6.49 \\
.18 \\
-2.49 \\
-3.95 \\
-6.34\end{array}$ & $\begin{array}{l}.6628 \\
.9366 \\
.9207 \\
.8638 \\
.7105\end{array}$ & $\begin{array}{r}.8837 \\
1.2832 \\
1.3584 \\
1.3014 \\
1.0533\end{array}$ & $\begin{array}{l}.3767 \\
.6189 \\
.5089 \\
.4562 \\
.3808\end{array}$ \\
\hline $\begin{array}{l}155 \\
156 \\
157 \\
158 \\
159\end{array}$ & $\begin{array}{l}24.51 \\
26.21 \\
24.07 \\
21.92 \\
24.67\end{array}$ & $\begin{array}{l}34.32 \\
33.87 \\
31.14 \\
32.18 \\
35.06\end{array}$ & $\begin{array}{r}9.79 \\
15.21 \\
18.26 \\
8.97 \\
14.48\end{array}$ & $\begin{array}{r}-1.57 \\
.02 \\
1.22 \\
-1.29 \\
-.84\end{array}$ & $\begin{array}{l}5.20 \\
4.89 \\
6.09 \\
4.79 \\
5.31\end{array}$ & $\begin{array}{r}-11.21 \\
-7.07 \\
-5.44 \\
-8.69 \\
-7.77\end{array}$ & $\begin{array}{l}.5817 \\
.6363 \\
.6862 \\
.5875 \\
.6107\end{array}$ & $\begin{array}{l}.8844 \\
.8652 \\
.9402 \\
.8593 \\
.8910\end{array}$ & $\begin{array}{l}.2602 \\
.3599 \\
.4078 \\
.3199 \\
.3411\end{array}$ \\
\hline $\begin{array}{l}160 \\
161 \\
162 \\
163 \\
164\end{array}$ & $\begin{array}{l}25.12 \\
27.44 \\
29.33 \\
30.68 \\
30.26\end{array}$ & $\begin{array}{l}38.23 \\
39.89 \\
39.54 \\
38.16 \\
36.44\end{array}$ & $\begin{array}{r}9.42 \\
11.73 \\
14.58 \\
21.40 \\
21.53\end{array}$ & $\begin{array}{r}-.63 \\
.50 \\
2.12 \\
3.32 \\
2.95\end{array}$ & $\begin{array}{l}6.02 \\
7.85 \\
8.90 \\
8.51 \\
7.40\end{array}$ & $\begin{array}{l}-9.67 \\
-8.70 \\
-6.33 \\
-3.11 \\
-1.72\end{array}$ & $\begin{array}{l}.6277 \\
.6860 \\
.7579 \\
.8028 \\
.7741\end{array}$ & $\begin{array}{r}.9361 \\
1.0615 \\
1.1395 \\
1.1095 \\
1.0289\end{array}$ & $\begin{array}{l}.2940 \\
.3171 \\
.3808 \\
.4859 \\
.5386\end{array}$ \\
\hline $\begin{array}{l}165 \\
166 \\
167 \\
168 \\
169\end{array}$ & $\begin{array}{l}28.80 \\
25.71 \\
23.57 \\
27.04 \\
27.11\end{array}$ & $\begin{array}{l}35.59 \\
32.56 \\
31.71 \\
34.90 \\
35.75\end{array}$ & $\begin{array}{l}22.72 \\
19.07 \\
12.69 \\
14.33 \\
18.03\end{array}$ & $\begin{array}{r}3.93 \\
1.32 \\
-1.39 \\
-.29 \\
-.54\end{array}$ & $\begin{array}{l}8.95 \\
6.02 \\
4.67 \\
4.79 \\
5.08\end{array}$ & $\begin{array}{l}-1.53 \\
-4.71 \\
-8.59 \\
-6.84 \\
-7.42\end{array}$ & $\begin{array}{l}.8341 \\
.6896 \\
.5791 \\
.6211 \\
.6179\end{array}$ & $\begin{array}{r}1.1433 \\
.9361 \\
.8519 \\
.8593 \\
.8765\end{array}$ & $\begin{array}{l}.5460 \\
.4309 \\
.3199 \\
.3664 \\
.3503\end{array}$ \\
\hline $\begin{array}{l}170 \\
171 \\
172 \\
173 \\
174\end{array}$ & $\begin{array}{l}26: 70 \\
27.91 \\
30.53 \\
30.86 \\
30.75\end{array}$ & $\begin{array}{l}37.19 \\
37.47 \\
38.03 \\
38.37 \\
39.82\end{array}$ & $\begin{array}{l}10.66 \\
11.48 \\
21.62 \\
18.08 \\
16.16\end{array}$ & $\begin{array}{r}-.11 \\
.93 \\
5.28 \\
3.79 \\
1.91\end{array}$ & $\begin{array}{l}6.77 \\
6.89 \\
9.56 \\
9.11 \\
7.43\end{array}$ & $\begin{array}{r}-8.96 \\
-8.81 \\
.93 \\
-2.97 \\
-4.43\end{array}$ & $\begin{array}{l}.6514 \\
.6981 \\
.9113 \\
.8320 \\
.7329\end{array}$ & $\begin{array}{r}.9858 \\
.9935 \\
1.1915 \\
1.1558 \\
1.0309\end{array}$ & $\begin{array}{l}.3108 \\
.3215 \\
.6531 \\
.4912 \\
.4403\end{array}$ \\
\hline $\begin{array}{l}175 \\
176 \\
177 \\
178 \\
179\end{array}$ & $\begin{array}{l}29.40 \\
32.79 \\
35.18 \\
33.36 \\
30.78\end{array}$ & $\begin{array}{l}40.72 \\
41.19 \\
42.56 \\
41.91 \\
42.53\end{array}$ & $\begin{array}{l}11.65 \\
17.85 \\
28.17 \\
22.17 \\
18.19\end{array}$ & $\begin{array}{l}1.40 \\
2.61 \\
3.15 \\
5.10 \\
6.45\end{array}$ & $\begin{array}{r}8.53 \\
7.71 \\
7.53 \\
10.48 \\
13.67\end{array}$ & $\begin{array}{r}-8.42 \\
-5.50 \\
-.89 \\
-1.69 \\
-1.46\end{array}$ & $\begin{array}{r}.7308 \\
.7639 \\
.7823 \\
.9162 \\
1.0279\end{array}$ & $\begin{array}{l}1.1115 \\
1.0513 \\
1.0381 \\
1.2674 \\
1.5633\end{array}$ & $\begin{array}{l}.3242 \\
.4059 \\
.5725 \\
.5399 \\
.5490\end{array}$ \\
\hline $\begin{array}{l}180 \\
181 \\
182 \\
183 \\
184\end{array}$ & $\begin{array}{l}33.08 \\
33.17 \\
32.76 \\
32.21 \\
28.22\end{array}$ & $\begin{array}{l}42.87 \\
42.98 \\
38.28 \\
39.01 \\
36.99\end{array}$ & $\begin{array}{l}19.34 \\
19.59 \\
25.79 \\
22.49 \\
12.85\end{array}$ & $\begin{array}{l}7.19 \\
6.26 \\
6.77 \\
6.05 \\
2.83\end{array}$ & $\begin{array}{r}13.38 \\
12.02 \\
10.97 \\
11.11 \\
9.15\end{array}$ & $\begin{array}{r}.05 \\
-1.43 \\
1.70 \\
.24 \\
-7.03\end{array}$ & $\begin{array}{r}1.0606 \\
.9908 \\
1.0051 \\
.9634 \\
.7917\end{array}$ & $\begin{array}{l}1.5342 \\
1.4030 \\
1.3096 \\
1.3212 \\
1.1587\end{array}$ & $\begin{array}{l}.6129 \\
.5501 \\
.6905 \\
.6220 \\
.3610\end{array}$ \\
\hline $\begin{array}{l}185 \\
186 \\
187 \\
188 \\
189\end{array}$ & $\begin{array}{l}28.86 \\
28.40 \\
27.21 \\
29.18 \\
27.73\end{array}$ & $\begin{array}{l}36.08 \\
35.82 \\
37.76 \\
37.94 \\
40.18\end{array}$ & $\begin{array}{l}19.11 \\
17.89 \\
15.03 \\
18.46 \\
13.73\end{array}$ & $\begin{array}{l}5.71 \\
5.94 \\
3.61 \\
4.78 \\
5.30\end{array}$ & $\begin{array}{r}10.79 \\
11.13 \\
9.69 \\
10.86 \\
12.86\end{array}$ & $\begin{array}{r}-1.65 \\
-.62 \\
-3.40 \\
-3.79 \\
-4.55\end{array}$ & $\begin{array}{l}.9529 \\
.9642 \\
.8319 \\
.9025 \\
.9668\end{array}$ & $\begin{array}{l}1.2934 \\
1.3234 \\
1.2843 \\
1.2996 \\
1.4828\end{array}$ & $\begin{array}{l}.5414 \\
.5837 \\
.4758 \\
.4619 \\
.4361\end{array}$ \\
\hline $\begin{array}{l}190 \\
191 \\
192 \\
193 \\
194\end{array}$ & $\begin{array}{l}30.42 \\
31.66 \\
28.92 \\
29.39 \\
29.74\end{array}$ & $\begin{array}{l}39.83 \\
38.53 \\
38.44 \\
40.04 \\
39.47\end{array}$ & $\begin{array}{l}14.37 \\
21.38 \\
15.43 \\
13.91 \\
18.27\end{array}$ & $\begin{array}{l}6.56 \\
7.72 \\
6.86 \\
7.53 \\
7.20\end{array}$ & $\begin{array}{l}13.19 \\
12.52 \\
13.13 \\
14.39 \\
13.35\end{array}$ & $\begin{array}{r}-1.88 \\
.83 \\
-1.61 \\
-1.32 \\
.10\end{array}$ & $\begin{array}{l}1.0342 \\
1.0859 \\
1.0443 \\
1.1050 \\
1.0638\end{array}$ & $\begin{array}{l}1.5157 \\
1.4505 \\
1.5094 \\
1.6381 \\
1.5310\end{array}$ & $\begin{array}{l}.5325 \\
.6493 \\
.5433 \\
.5549 \\
.6153\end{array}$ \\
\hline
\end{tabular}


Table 5. Daily mean, maximum, and minimum air and dew-point temperatures and vapor pressure, measured by energy-budget station at Rogers Spring Site 2, 1994-Continued

\begin{tabular}{|c|c|c|c|c|c|c|c|c|c|}
\hline \multirow{2}{*}{$\begin{array}{l}\text { Calendar } \\
\text { day }\end{array}$} & \multicolumn{3}{|c|}{$\begin{array}{l}\text { Air temperature at } 1.25 \text { meters, } \\
\text { in degrees Celslus }\end{array}$} & \multicolumn{3}{|c|}{$\begin{array}{l}\text { Dew-point temperature at } 1.25 \\
\text { meters, In degrees Celsius }\end{array}$} & \multicolumn{3}{|c|}{$\begin{array}{c}\text { Vapor pressure at } 1.25 \text { meters, } \\
\text { in kilopascals }\end{array}$} \\
\hline & Mean & Maximum & Minimum & Mean & Maximum & Minimum & Mean & Maximum & Minimum \\
\hline $\begin{array}{l}195 \\
196 \\
197 \\
198 \\
199\end{array}$ & $\begin{array}{l}30.32 \\
30.47 \\
30.48 \\
31.30 \\
31.59\end{array}$ & $\begin{array}{l}39.18 \\
38.64 \\
38.20 \\
39.17 \\
37.12\end{array}$ & $\begin{array}{l}15.46 \\
18.99 \\
18.81 \\
22.95 \\
24.35\end{array}$ & $\begin{array}{r}6.99 \\
7.41 \\
6.68 \\
9.15 \\
11.10\end{array}$ & $\begin{array}{l}13.39 \\
13.39 \\
11.50 \\
14.42 \\
14.68\end{array}$ & $\begin{array}{r}-0.38 \\
-.27 \\
-.56 \\
4.14 \\
6.00\end{array}$ & $\begin{array}{l}1.0537 \\
1.0785 \\
1.0102 \\
1.1885 \\
1.3409\end{array}$ & $\begin{array}{l}1.5349 \\
1.5355 \\
1.3560 \\
1.6418 \\
1.6695\end{array}$ & $\begin{array}{r}0.5943 \\
.5990 \\
.5865 \\
.8209 \\
.9345\end{array}$ \\
\hline $\begin{array}{l}200 \\
201 \\
202 \\
203 \\
204\end{array}$ & $\begin{array}{l}29.06 \\
28.32 \\
29.48 \\
30.96 \\
30.43\end{array}$ & $\begin{array}{l}36.24 \\
36.62 \\
37.03 \\
38.04 \\
38.47\end{array}$ & $\begin{array}{l}21.68 \\
19.59 \\
18.48 \\
22.57 \\
17.69\end{array}$ & $\begin{array}{r}11.95 \\
13.36 \\
12.59 \\
11.11 \\
9.88\end{array}$ & $\begin{array}{l}16.53 \\
18.55 \\
18.10 \\
15.88 \\
15.17\end{array}$ & $\begin{array}{l}7.04 \\
8.29 \\
6.89 \\
6.81 \\
3.79\end{array}$ & $\begin{array}{l}1.4250 \\
1.5702 \\
1.5052 \\
1.3559 \\
1.2609\end{array}$ & $\begin{array}{l}1.8795 \\
2.1359 \\
2.0761 \\
1.8035 \\
1.7234\end{array}$ & $\begin{array}{r}1.0039 \\
1.0931 \\
.9937 \\
.9884 \\
.8014\end{array}$ \\
\hline $\begin{array}{l}205 \\
206 \\
207 \\
208 \\
209\end{array}$ & $\begin{array}{l}31.06 \\
30.27 \\
29.12 \\
30.71 \\
31.45\end{array}$ & $\begin{array}{l}38.60 \\
39.79 \\
40.35 \\
40.96 \\
40.88\end{array}$ & $\begin{array}{l}22.32 \\
16.17 \\
14.59 \\
15.26 \\
19.46\end{array}$ & $\begin{array}{l}8.84 \\
6.08 \\
5.60 \\
6.43 \\
7.35\end{array}$ & $\begin{array}{l}14.58 \\
12.62 \\
12.63 \\
12.93 \\
13.34\end{array}$ & $\begin{array}{r}1.85 \\
-1.47 \\
-2.57 \\
-1.65 \\
.83\end{array}$ & $\begin{array}{r}1.1807 \\
.9895 \\
.9641 \\
1.0174 \\
1.0703\end{array}$ & $\begin{array}{l}1.6588 \\
1.4598 \\
1.4610 \\
1.4903 \\
1.5302\end{array}$ & $\begin{array}{l}.6986 \\
.5490 \\
.5057 \\
.5414 \\
.6484\end{array}$ \\
\hline $\begin{array}{l}210 \\
211 \\
212 \\
213 \\
214\end{array}$ & $\begin{array}{l}32.37 \\
32.64 \\
31.39 \\
32.03 \\
30.92\end{array}$ & $\begin{array}{l}39.23 \\
38.61 \\
38.68 \\
39.03 \\
38.89\end{array}$ & $\begin{array}{l}21.55 \\
27.18 \\
22.68 \\
22.29 \\
19.33\end{array}$ & $\begin{array}{r}9.41 \\
9.75 \\
6.51 \\
5.26 \\
2.91\end{array}$ & $\begin{array}{r}13.64 \\
13.72 \\
11.46 \\
10.00 \\
8.18\end{array}$ & $\begin{array}{r}3.51 \\
5.00 \\
1.64 \\
.42 \\
-3.42\end{array}$ & $\begin{array}{r}1.2155 \\
1.2269 \\
.9969 \\
.9128 \\
.7785\end{array}$ & $\begin{array}{l}1.6987 \\
1.5688 \\
1.3525 \\
1.2268 \\
1.0853\end{array}$ & $\begin{array}{l}.7856 \\
.8722 \\
.6875 \\
.6296 \\
.4751\end{array}$ \\
\hline $\begin{array}{l}215 \\
216 \\
217 \\
218 \\
219\end{array}$ & $\begin{array}{l}27.84 \\
27.33 \\
30.78 \\
33.29 \\
32.51\end{array}$ & $\begin{array}{l}39.52 \\
39.36 \\
41.05 \\
42.58 \\
40.69\end{array}$ & $\begin{array}{l}12.47 \\
12.46 \\
14.67 \\
19.35 \\
18.25\end{array}$ & $\begin{array}{r}.45 \\
-.91 \\
.19 \\
4.25 \\
5.53\end{array}$ & $\begin{array}{r}7.78 \\
7.28 \\
6.64 \\
10.08 \\
10.69\end{array}$ & $\begin{array}{l}-6.63 \\
-9.57 \\
-8.74 \\
-3.35 \\
-1.99\end{array}$ & $\begin{array}{l}.6775 \\
.6260 \\
.6602 \\
.8596 \\
.9391\end{array}$ & $\begin{array}{r}1.1936 \\
1.0207 \\
.9765 \\
1.2333 \\
1.2852\end{array}$ & $\begin{array}{l}.3723 \\
.2963 \\
.3163 \\
.4774 \\
.5281\end{array}$ \\
\hline $\begin{array}{l}220 \\
221 \\
222 \\
223 \\
224\end{array}$ & $\begin{array}{l}32.03 \\
31.33 \\
30.65 \\
31.81 \\
32.86\end{array}$ & $\begin{array}{l}36.06 \\
36.85 \\
37.76 \\
39.77 \\
38.27\end{array}$ & $\begin{array}{l}27.84 \\
26.43 \\
22.63 \\
23.13 \\
25.88\end{array}$ & $\begin{array}{r}6.53 \\
10.66 \\
12.12 \\
11.82 \\
11.81\end{array}$ & $\begin{array}{l}10.19 \\
14.55 \\
16.88 \\
16.79 \\
15.92\end{array}$ & $\begin{array}{l}3.20 \\
6.57 \\
7.55 \\
6.20 \\
6.44\end{array}$ & $\begin{array}{r}.9817 \\
1.3023 \\
1.4429 \\
1.4201 \\
1.4052\end{array}$ & $\begin{array}{l}1.2430 \\
1.6558 \\
1.9222 \\
1.9102 \\
1.8079\end{array}$ & $\begin{array}{r}.7686 \\
.9723 \\
1.0394 \\
.9475 \\
.9632\end{array}$ \\
\hline $\begin{array}{l}225 \\
226 \\
227 \\
228 \\
229\end{array}$ & $\begin{array}{l}31.32 \\
29.92 \\
30.25 \\
33.33 \\
30.66\end{array}$ & $\begin{array}{l}39.01 \\
39.95 \\
41.38 \\
41.35 \\
37.12\end{array}$ & $\begin{array}{l}25.22 \\
19.83 \\
18.07 \\
22.20 \\
22.55\end{array}$ & $\begin{array}{r}12.55 \\
12.08 \\
9.99 \\
9.49 \\
9.76\end{array}$ & $\begin{array}{l}17.36 \\
17.72 \\
15.70 \\
14.18 \\
14.12\end{array}$ & $\begin{array}{l}8.92 \\
6.57 \\
4.55 \\
3.08 \\
3.98\end{array}$ & $\begin{array}{l}1.4794 \\
1.4602 \\
1.2697 \\
1.2220 \\
1.2346\end{array}$ & $\begin{array}{l}1.9816 \\
2.0266 \\
1.7846 \\
1.6161 \\
1.6101\end{array}$ & $\begin{array}{r}1.1409 \\
.9721 \\
.8450 \\
.7619 \\
.8121\end{array}$ \\
\hline $\begin{array}{l}230 \\
231 \\
232 \\
233 \\
234\end{array}$ & $\begin{array}{l}31.55 \\
31.02 \\
30.80 \\
30.26 \\
28.09\end{array}$ & $\begin{array}{l}36.99 \\
37.65 \\
38.47 \\
36.84 \\
35.12\end{array}$ & $\begin{array}{l}25.24 \\
21.69 \\
18.94 \\
21.85 \\
20.86\end{array}$ & $\begin{array}{r}11.38 \\
10.97 \\
10.22 \\
5.38 \\
2.67\end{array}$ & $\begin{array}{r}14.62 \\
15.50 \\
15.29 \\
10.37 \\
8.14\end{array}$ & $\begin{array}{r}7.99 \\
5.92 \\
4.94 \\
.73 \\
-5.55\end{array}$ & $\begin{array}{r}1.3600 \\
1.3368 \\
1.2828 \\
.9182 \\
.7740\end{array}$ & $\begin{array}{l}1.6626 \\
1.7598 \\
1.7357 \\
1.2581 \\
1.0819\end{array}$ & $\begin{array}{r}1.0716 \\
.9295 \\
.8685 \\
.6440 \\
.4044\end{array}$ \\
\hline $\begin{array}{l}235 \\
236 \\
237 \\
238 \\
239\end{array}$ & $\begin{array}{l}24.50 \\
26.75 \\
30.51 \\
29.98 \\
29.31\end{array}$ & $\begin{array}{l}36.44 \\
38.53 \\
38.40 \\
38.19 \\
36.43\end{array}$ & $\begin{array}{l}12.44 \\
12.02 \\
20.38 \\
18.25 \\
24.26\end{array}$ & $\begin{array}{r}-1.90 \\
-1.88 \\
.28 \\
2.12 \\
8.62\end{array}$ & $\begin{array}{r}5.94 \\
5.97 \\
5.71 \\
6.47 \\
14.64\end{array}$ & $\begin{array}{r}-10.13 \\
-10.14 \\
-6.02 \\
-6.26 \\
4.40\end{array}$ & $\begin{array}{r}.5790 \\
.5739 \\
.6456 \\
.7452 \\
1.1468\end{array}$ & $\begin{array}{r}.9305 \\
.9325 \\
.9160 \\
.9656 \\
1.6651\end{array}$ & $\begin{array}{l}.2833 \\
.2832 \\
.3902 \\
.3831 \\
.8366\end{array}$ \\
\hline $\begin{array}{l}240 \\
241 \\
242 \\
243 \\
244\end{array}$ & $\begin{array}{l}30.13 \\
27.36 \\
23.80 \\
25.57 \\
27.24\end{array}$ & $\begin{array}{l}37.88 \\
37.03 \\
36.74 \\
35.66 \\
35.07\end{array}$ & $\begin{array}{r}19.92 \\
13.72 \\
9.31 \\
11.01 \\
17.47\end{array}$ & $\begin{array}{r}6.47 \\
1.91 \\
-2.18 \\
-1.43 \\
-.49\end{array}$ & $\begin{array}{r}11.62 \\
8.30 \\
5.96 \\
6.28 \\
5.33\end{array}$ & $\begin{array}{r}1.84 \\
-8.20 \\
-11.03 \\
-10.34 \\
-6.80\end{array}$ & $\begin{array}{l}.9953 \\
.7483 \\
.5726 \\
.5925 \\
.6183\end{array}$ & $\begin{array}{r}1.3665 \\
1.2828 \\
.9320 \\
.9530 \\
.8920\end{array}$ & $\begin{array}{l}.6973 \\
.3299 \\
.2640 \\
.2789 \\
.3676\end{array}$ \\
\hline
\end{tabular}


Table 5. Daily mean, maximum, and minimum air and dew-point temperatures and vapor pressure, measured by energy-budget station at Rogers Spring Site 2, 1994-Continued

\begin{tabular}{|c|c|c|c|c|c|c|c|c|c|}
\hline \multirow{2}{*}{$\begin{array}{l}\text { Calendar } \\
\text { day }\end{array}$} & \multicolumn{3}{|c|}{$\begin{array}{l}\text { Air temperature at } 1.25 \text { meters, } \\
\text { in degrees Celsius }\end{array}$} & \multicolumn{3}{|c|}{$\begin{array}{l}\text { Dew-point temperature at } 1.25 \\
\text { meters, in degrees Celsius }\end{array}$} & \multicolumn{3}{|c|}{$\begin{array}{l}\text { Vapor pressure at } 1.25 \text { meters, } \\
\text { in kilopascals }\end{array}$} \\
\hline & Mean & Maximum & Minimum & Mean & Maximum & Minimum & Mean & Maximum & Minimum \\
\hline $\begin{array}{l}245 \\
246 \\
247 \\
248 \\
256\end{array}$ & $\begin{array}{l}27.33 \\
23.15 \\
23.32 \\
25.69 \\
16.74\end{array}$ & $\begin{array}{l}34.50 \\
33.62 \\
35.52 \\
36.74 \\
26.14\end{array}$ & $\begin{array}{r}15.79 \\
10.86 \\
9.73 \\
13.95 \\
7.36\end{array}$ & $\begin{array}{r}-1.35 \\
-3.03 \\
-.24 \\
4.51 \\
-3.30\end{array}$ & $\begin{array}{r}3.30 \\
4.08 \\
8.34 \\
11.89 \\
4.11\end{array}$ & $\begin{array}{r}-8.03 \\
-11.49 \\
-9.73 \\
-3.92 \\
-10.90\end{array}$ & $\begin{array}{r}0.5718 \\
.5233 \\
.6605 \\
.9027 \\
.5133\end{array}$ & $\begin{array}{r}0.7740 \\
.8175 \\
1.0967 \\
1.3913 \\
.8192\end{array}$ & $\begin{array}{r}0.3342 \\
.2544 \\
.2925 \\
.4573 \\
.2665\end{array}$ \\
\hline $\begin{array}{l}257 \\
258 \\
259 \\
260 \\
261\end{array}$ & $\begin{array}{l}18.87 \\
21.16 \\
21.24 \\
21.68 \\
22.00\end{array}$ & $\begin{array}{l}30.05 \\
33.25 \\
33.15 \\
33.47 \\
34.18\end{array}$ & $\begin{array}{r}9.56 \\
8.16 \\
7.52 \\
10.23 \\
11.76\end{array}$ & $\begin{array}{r}-1.75 \\
-1.71 \\
-2.30 \\
-2.01 \\
.01\end{array}$ & $\begin{array}{l}6.07 \\
6.51 \\
4.57 \\
5.91 \\
8.01\end{array}$ & $\begin{array}{r}-8.46 \\
-9.82 \\
-10.36 \\
-9.65 \\
-7.68\end{array}$ & $\begin{array}{l}.5798 \\
.5901 \\
.5578 \\
.5676 \\
.6536\end{array}$ & $\begin{array}{r}.9390 \\
.9678 \\
.9256 \\
.9291 \\
1.0727\end{array}$ & $\begin{array}{l}.3231 \\
.2905 \\
.2783 \\
.2943 \\
.3433\end{array}$ \\
\hline $\begin{array}{l}262 \\
263 \\
264 \\
265 \\
266\end{array}$ & $\begin{array}{l}22.00 \\
23.87 \\
23.01 \\
22.03 \\
22.49\end{array}$ & $\begin{array}{l}30.47 \\
32.40 \\
33.55 \\
34.69 \\
34.93\end{array}$ & $\begin{array}{r}13.68 \\
14.77 \\
12.71 \\
9.17 \\
10.28\end{array}$ & $\begin{array}{l}2.48 \\
5.55 \\
6.06 \\
6.35 \\
6.22\end{array}$ & $\begin{array}{r}9.42 \\
11.81 \\
14.66 \\
15.36 \\
15.18\end{array}$ & $\begin{array}{r}-3.77 \\
-.59 \\
-2.40 \\
-1.89 \\
-2.52\end{array}$ & $\begin{array}{r}.7652 \\
.9443 \\
1.0092 \\
1.0395 \\
1.0277\end{array}$ & $\begin{array}{l}1.1804 \\
1.3841 \\
1.6670 \\
1.7443 \\
1.7244\end{array}$ & $\begin{array}{l}.4626 \\
.5853 \\
.5122 \\
.5320 \\
.5076\end{array}$ \\
\hline $\begin{array}{l}267 \\
268 \\
269 \\
270 \\
271\end{array}$ & $\begin{array}{l}24.44 \\
21.33 \\
21.43 \\
23.39 \\
25.20\end{array}$ & $\begin{array}{l}32.35 \\
33.60 \\
35.18 \\
35.07 \\
32.35\end{array}$ & $\begin{array}{r}16.34 \\
10.58 \\
9.05 \\
9.05 \\
13.49\end{array}$ & $\begin{array}{l}9.33 \\
7.09: \\
3.75 \\
3.67 \\
6.85\end{array}$ & $\begin{array}{l}15.95 \\
16.31 \\
12.33 \\
11.20 \\
12.42\end{array}$ & $\begin{array}{r}3.77 \\
-.43 \\
-2.93 \\
-5.27 \\
-1.17\end{array}$ & $\begin{array}{r}1.2194 \\
1.0935 \\
.8554 \\
.8492 \\
1.0186\end{array}$ & $\begin{array}{l}1.8114 \\
1.8535 \\
1.4326 \\
1.3292 \\
1.4406\end{array}$ & $\begin{array}{l}.8000 \\
.5919 \\
.4924 \\
.4129 \\
.4684\end{array}$ \\
\hline $\begin{array}{l}272 \\
273 \\
274 \\
275 \\
276\end{array}$ & $\begin{array}{l}22.59 \\
18.77 \\
18.47 \\
21.38 \\
19.08\end{array}$ & $\begin{array}{l}30.35 \\
28.19 \\
29.59 \\
31.43 \\
23.51\end{array}$ & $\begin{array}{r}14.80 \\
9.31 \\
7.47 \\
9.18 \\
11.27\end{array}$ & $\begin{array}{r}6.91 \\
2.57 \\
2.23 \\
3.60 \\
.14\end{array}$ & $\begin{array}{r}12.89 \\
9.39 \\
10.68 \\
11.08 \\
3.78\end{array}$ & $\begin{array}{r}-.03 \\
-4.71 \\
-5.63 \\
-4.54 \\
-2.64\end{array}$ & $\begin{array}{r}1.0263 \\
.7743 \\
.7796 \\
.8395 \\
.6251\end{array}$ & $\begin{array}{r}1.4863 \\
1.1776 \\
1.2839 \\
1.3190 \\
.8005\end{array}$ & $\begin{array}{l}.6099 \\
.4308 \\
.4020 \\
.4364 \\
.5034\end{array}$ \\
\hline $\begin{array}{l}277 \\
278 \\
279 \\
280 \\
281\end{array}$ & $\begin{array}{l}18.81 \\
14.75 \\
14.57 \\
18.22 \\
19.21\end{array}$ & $\begin{array}{l}21.87 \\
21.49 \\
25.07 \\
27.82 \\
28.62\end{array}$ & $\begin{array}{r}16.04 \\
5.66 \\
2.65 \\
6.10 \\
7.66\end{array}$ & $\begin{array}{l}2.60 \\
1.52 \\
-.17 \\
-- \\
-.20\end{array}$ & $\begin{array}{l}5.56 \\
7.33 \\
8.52 \\
- \\
6.71\end{array}$ & $\begin{array}{r}-.01 \\
-6.01 \\
-8.89 \\
-7.49 \\
-8.37\end{array}$ & $\begin{array}{r}.7427 \\
.7076 \\
.6600 \\
-\quad .6379\end{array}$ & $\begin{array}{r}.9065 \\
1.0242 \\
1.1105 \\
-- \\
.9813\end{array}$ & $\begin{array}{l}.6103 \\
.3906 \\
.3151 \\
.3484 \\
.3256\end{array}$ \\
\hline $\begin{array}{l}282 \\
283 \\
284 \\
285 \\
286\end{array}$ & $\begin{array}{l}16.28 \\
17.16 \\
18.65 \\
19.66 \\
15.70\end{array}$ & $\begin{array}{l}29.63 \\
29.23 \\
28.43 \\
27.46 \\
23.29\end{array}$ & $\begin{array}{l}4.15 \\
5.97 \\
6.88 \\
6.50 \\
5.31\end{array}$ & $\begin{array}{l}-2.75 \\
-2.93 \\
-1.43 \\
-1.13 \\
-1.68\end{array}$ & $\begin{array}{l}6.80 \\
6.00 \\
6.05 \\
5.36 \\
5.24\end{array}$ & $\begin{array}{r}-11.55 \\
-11.22 \\
-9.16 \\
-9.27 \\
-10.45\end{array}$ & $\begin{array}{l}.5578 \\
.5420 \\
.5868 \\
.5965 \\
.5731\end{array}$ & $\begin{array}{l}.9878 \\
.9344 \\
.9377 \\
.8940 \\
.8865\end{array}$ & $\begin{array}{l}.2531 \\
.2599 \\
.3061 \\
.3035 \\
.2765\end{array}$ \\
\hline $\begin{array}{l}287 \\
288 \\
289 \\
290 \\
291\end{array}$ & $\begin{array}{l}17.07 \\
13.16 \\
11.52 \\
10.50 \\
11.48\end{array}$ & $\begin{array}{l}21.93 \\
18.52 \\
19.69 \\
20.37 \\
22.96\end{array}$ & $\begin{array}{r}12.06 \\
5.27 \\
2.63 \\
1.63 \\
.86\end{array}$ & $\begin{array}{r}.83 \\
-2.49 \\
-4.41 \\
-3.45 \\
-6.73\end{array}$ & $\begin{array}{l}5.74 \\
1.76 \\
2.02 \\
4.71 \\
-.41\end{array}$ & $\begin{array}{r}-3.20 \\
-9.40 \\
-10.14 \\
-11.27 \\
-10.62\end{array}$ & $\begin{array}{l}.6633 \\
.5220 \\
.4611 \\
.5120 \\
.3841\end{array}$ & $\begin{array}{l}.9177 \\
.6934 \\
.7064 \\
.8541 \\
.5932\end{array}$ & $\begin{array}{l}.4825 \\
.3047 \\
.2833 \\
.2590 \\
.2726\end{array}$ \\
\hline $\begin{array}{l}292 \\
293 \\
294 \\
295 \\
296\end{array}$ & $\begin{array}{l}11.90 \\
12.17 \\
12.17 \\
12.39 \\
14.27\end{array}$ & $\begin{array}{l}24.29 \\
24.83 \\
26.45 \\
25.59 \\
25.75\end{array}$ & $\begin{array}{r}2.08 \\
2.03 \\
-.60 \\
1.77 \\
.37\end{array}$ & $\begin{array}{l}-7.52 \\
-3.80 \\
-7.41 \\
-8.36 \\
-8.22\end{array}$ & $\begin{array}{r}-1.12 \\
3.90 \\
-.96 \\
-2.24 \\
-2.45\end{array}$ & $\begin{array}{l}-12.58 \\
-11.17 \\
-13.39 \\
-14.18 \\
-15.30\end{array}$ & $\begin{array}{l}.3651 \\
.4977 \\
.3732 \\
.3438 \\
.3471\end{array}$ & $\begin{array}{l}.5629 \\
.8675 \\
.5695 \\
.5182 \\
.5110\end{array}$ & $\begin{array}{l}.2330 \\
.2610 \\
.2182 \\
.2046 \\
.1865\end{array}$ \\
\hline $\begin{array}{l}297 \\
298 \\
299 \\
300 \\
301\end{array}$ & $\begin{array}{l}15.77 \\
14.57 \\
14.38 \\
14.47 \\
13.20\end{array}$ & $\begin{array}{l}26.70 \\
27.16 \\
27.16 \\
27.04 \\
26.26\end{array}$ & $\begin{array}{l}6.14 \\
4.95 \\
3.59 \\
3.92 \\
3.56\end{array}$ & $\begin{array}{l}-7.96 \\
-2.75 \\
-2.43 \\
-3.14 \\
-3.66\end{array}$ & $\begin{array}{r}-3.32 \\
7.15 \\
6.71 \\
6.03 \\
5.33\end{array}$ & $\begin{array}{r}-12.33 \\
-13.37 \\
-9.67 \\
-9.48 \\
-9.95\end{array}$ & $\begin{array}{l}.3437 \\
.5739 \\
.5598 \\
.5265 \\
.5083\end{array}$ & $\begin{array}{r}.4782 \\
1.0113 \\
.9816 \\
.9365 \\
.8920\end{array}$ & $\begin{array}{l}.2378 \\
.2186 \\
.2947 \\
.2984 \\
.2875\end{array}$ \\
\hline
\end{tabular}


Table 5. Daily mean, maximum, and minimum air and dew-point temperatures and vapor pressure, measured by energy-budget station at Rogers Spring Site 2, 1994-Continued

\begin{tabular}{|c|c|c|c|c|c|c|c|c|c|}
\hline \multirow{2}{*}{$\begin{array}{l}\text { Calendar } \\
\text { day }\end{array}$} & \multicolumn{3}{|c|}{$\begin{array}{l}\text { Air temperature at } 1.25 \text { meters, } \\
\text { in degrees Celslus }\end{array}$} & \multicolumn{3}{|c|}{$\begin{array}{l}\text { Dew-polnt temperature at } 1.25 \\
\text { meters, in degrees Celsius }\end{array}$} & \multicolumn{3}{|c|}{$\begin{array}{c}\text { Vapor pressure at } 1.25 \text { meters, } \\
\text { in kilopascals }\end{array}$} \\
\hline & Mean & Maximum & Minimum & Mean & Maximum & Minimum & Mean & Maximum & Minimum \\
\hline $\begin{array}{l}302 \\
303 \\
304 \\
305 \\
306\end{array}$ & $\begin{array}{l}14.18 \\
13.50 \\
10.72 \\
15.31 \\
14.13\end{array}$ & $\begin{array}{l}25.64 \\
23.18 \\
24.68 \\
25.82 \\
18.14\end{array}$ & $\begin{array}{l}2.60 \\
3.61 \\
-.90 \\
1.08 \\
8.19\end{array}$ & $\begin{array}{l}-3.92 \\
-5.88 \\
-9.54 \\
-7.63 \\
-4.03\end{array}$ & $\begin{array}{l}4.66 \\
1.37 \\
-.69 \\
-.87 \\
-.84\end{array}$ & $\begin{array}{r}-11.47 \\
-14.25 \\
-15.67 \\
-17.00 \\
-7.38\end{array}$ & $\begin{array}{r}0.4981 \\
.4207 \\
.3277 \\
.3772 \\
.4580\end{array}$ & $\begin{array}{r}0.8516 \\
.6742 \\
.5810 \\
.5735 \\
.5745\end{array}$ & $\begin{array}{r}0.2547 \\
.2034 \\
.1810 \\
.1618 \\
.3514\end{array}$ \\
\hline $\begin{array}{l}307 \\
308 \\
309 \\
310 \\
311\end{array}$ & $\begin{array}{r}7.36 \\
4.60 \\
8.26 \\
12.31 \\
10.32\end{array}$ & $\begin{array}{l}12.27 \\
12.94 \\
18.25 \\
22.85 \\
19.06\end{array}$ & $\begin{array}{r}1.79 \\
-2.06 \\
-.49 \\
1.76 \\
2.93\end{array}$ & $\begin{array}{r}-7.37 \\
-11.10 \\
-9.85 \\
-7.64 \\
-8.83\end{array}$ & $\begin{array}{l}-3.30 \\
-4.62 \\
-3.32 \\
-1.31 \\
-3.16\end{array}$ & $\begin{array}{l}-12.98 \\
-16.40 \\
-14.85 \\
-13.51 \\
-13.88\end{array}$ & $\begin{array}{l}.3594 \\
.2766 \\
.3050 \\
.3628 \\
.3248\end{array}$ & $\begin{array}{l}.4792 \\
.4338 \\
.4784 \\
.5551 \\
.4842\end{array}$ & $\begin{array}{l}.2256 \\
.1702 \\
.1937 \\
.2162 \\
.2097\end{array}$ \\
\hline $\begin{array}{l}312 \\
313 \\
314 \\
315 \\
316\end{array}$ & $\begin{array}{r}10.29 \\
12.64 \\
10.03 \\
5.44 \\
5.07\end{array}$ & $\begin{array}{l}18.87 \\
20.07 \\
15.11 \\
12.98 \\
14.42\end{array}$ & $\begin{array}{r}3.48 \\
2.69 \\
2.00 \\
-3.44 \\
-3.55\end{array}$ & $\begin{array}{l}-4.86 \\
-2.01 \\
-1.95 \\
-4.06 \\
-4.24\end{array}$ & $\begin{array}{l}2.73 \\
4.04 \\
1.93 \\
2.99 \\
3.73\end{array}$ & $\begin{array}{r}-12.12 \\
-9.05 \\
-6.62 \\
-11.04 \\
-10.63\end{array}$ & $\begin{array}{l}.4601 \\
.5516 \\
.5360 \\
.4745 \\
.4766\end{array}$ & $\begin{array}{l}.7432 \\
.8152 \\
.7021 \\
.7572 \\
.7974\end{array}$ & $\begin{array}{l}.2420 \\
.3085 \\
.3728 \\
.2637 \\
.2726\end{array}$ \\
\hline $\begin{array}{l}317 \\
318 \\
319 \\
320\end{array}$ & $\begin{array}{l}7.45 \\
5.17 \\
5.23 \\
9.31\end{array}$ & $\begin{array}{l}13.08 \\
13.50 \\
15.24 \\
14.18\end{array}$ & $\begin{array}{r}3.09 \\
-3.70 \\
-8.20 \\
-1.31\end{array}$ & $\begin{array}{r}-4.60 \\
-7.70 \\
2.58 \\
10.24\end{array}$ & $\begin{array}{r}.56 \\
-1.15 \\
18.16 \\
17.95\end{array}$ & $\begin{array}{r}-8.19 \\
-15.00 \\
-17.93 \\
-.71\end{array}$ & $\begin{array}{l}.4452 \\
.3603 \\
1.0106 \\
1.3057\end{array}$ & $\begin{array}{r}.6362 \\
.5617 \\
2.0846 \\
2.0559\end{array}$ & $\begin{array}{l}.3300 \\
.1912 \\
.1497 \\
.5815\end{array}$ \\
\hline
\end{tabular}


Table 6. Daily mean, maximum, and minimum micrometeorological data measured by micromet station at Rogers Spring Site 2, 1994

\begin{tabular}{|c|c|c|c|c|c|c|c|c|c|c|c|c|}
\hline & \multirow{2}{*}{$\begin{array}{l}\text { Calendar } \\
\text { day }\end{array}$} & \multicolumn{2}{|c|}{$\begin{array}{l}\text { Net radiation, in watts } \\
\text { per square meter }\end{array}$} & \multicolumn{3}{|c|}{$\begin{array}{c}\text { Air temperature at } 1.25 \text { meters, } \\
\text { in degrees Celsius }\end{array}$} & \multicolumn{3}{|c|}{$\begin{array}{l}\text { Humidity at } 1.25 \text { meters, } \\
\text { in degrees Celsius }\end{array}$} & \multicolumn{3}{|c|}{$\begin{array}{l}\text { Windspeed at } 1.25 \text { meters, } \\
\text { in meters per second }\end{array}$} \\
\hline & & Mean & Maximum & Mean & Maximum & Minimum & Mean & Maximum & Minimum & Mean & Maximum & Minimum \\
\hline & $\begin{array}{l}1 \\
2 \\
3 \\
4 \\
5\end{array}$ & $\begin{array}{l}15.8 \\
28.6 \\
33.3 \\
32.3 \\
23.9\end{array}$ & $\begin{array}{l}208.5 \\
280.9 \\
279.4 \\
243.9 \\
272.6\end{array}$ & $\begin{array}{l}3.85 \\
5.57 \\
5.32 \\
7.25 \\
9.75\end{array}$ & $\begin{array}{l}13.68 \\
18.19 \\
18.30 \\
16.28 \\
20.09\end{array}$ & $\begin{array}{r}-2.74 \\
-4.14 \\
-4.78 \\
.02 \\
-.19\end{array}$ & $\begin{array}{l}53.2 \\
51.6 \\
48.8 \\
46.4 \\
36.8\end{array}$ & $\begin{array}{l}72.6 \\
81.6 \\
76.4 \\
69.4 \\
75.6\end{array}$ & $\begin{array}{l}26.4 \\
21.1 \\
18.9 \\
22.6 \\
17.7\end{array}$ & $\begin{array}{r}0.64 \\
.79 \\
.63 \\
.62 \\
1.82\end{array}$ & $\begin{array}{l}1.03 \\
1.33 \\
1.09 \\
1.05 \\
4.69\end{array}$ & $\begin{array}{r}0.23 \\
.30 \\
.20 \\
.31 \\
.44\end{array}$ \\
\hline & $\begin{array}{r}6 \\
7 \\
8 \\
9 \\
10\end{array}$ & $\begin{array}{l}11.7 \\
21.9 \\
24.9 \\
13.5 \\
26.2\end{array}$ & $\begin{array}{l}275.4 \\
249.5 \\
289.6 \\
217.7 \\
300.6\end{array}$ & $\begin{array}{r}6.36 \\
2.82 \\
.66 \\
2.64 \\
6.26\end{array}$ & $\begin{array}{l}13.54 \\
11.49 \\
12.89 \\
15.07 \\
15.11\end{array}$ & $\begin{array}{r}.56 \\
-6.13 \\
-7.08 \\
-7.44 \\
-3.21\end{array}$ & $\begin{array}{l}33.7 \\
46.9 \\
48.7 \\
47.5 \\
47.3\end{array}$ & $\begin{array}{l}52.7 \\
79.5 \\
75.0 \\
67.9 \\
80.4\end{array}$ & $\begin{array}{l}19.0 \\
20.8 \\
18.0 \\
25.9 \\
23.8\end{array}$ & $\begin{array}{r}1.82 \\
.76 \\
.72 \\
1.07 \\
1.51\end{array}$ & $\begin{array}{l}3.43 \\
1.37 \\
1.33 \\
2.86 \\
3.23\end{array}$ & $\begin{array}{l}.81 \\
.31 \\
.29 \\
.44 \\
.54\end{array}$ \\
\hline & $\begin{array}{l}11 \\
12 \\
13 \\
14 \\
15\end{array}$ & $\begin{array}{l}24.9 \\
27.7 \\
33.6 \\
21.7 \\
34.5\end{array}$ & $\begin{array}{l}286.7 \\
293.0 \\
280.3 \\
245.1 \\
296.8\end{array}$ & $\begin{array}{r}4.64 \\
8.61 \\
10.15 \\
11.69 \\
8.14\end{array}$ & $\begin{array}{l}15.58 \\
18.52 \\
21.40 \\
20.46 \\
19.95\end{array}$ & $\begin{array}{r}-6.32 \\
-.53 \\
-1.29 \\
4.32 \\
.23\end{array}$ & $\begin{array}{l}48.7 \\
38.3 \\
42.6 \\
44.4 \\
52.2\end{array}$ & $\begin{array}{l}80.5 \\
66.7 \\
63.7 \\
63.3 \\
79.7\end{array}$ & $\begin{array}{l}21.4 \\
18.0 \\
23.2 \\
23.8 \\
24.4\end{array}$ & $\begin{array}{r}.97 \\
1.42 \\
1.51 \\
1.53 \\
.68\end{array}$ & $\begin{array}{l}2.27 \\
3.60 \\
3.16 \\
2.68 \\
1.24\end{array}$ & $\begin{array}{l}.31 \\
.46 \\
.46 \\
.44 \\
.30\end{array}$ \\
\hline & $\begin{array}{l}16 \\
17 \\
18 \\
19 \\
20\end{array}$ & $\begin{array}{l}27.0 \\
31.4 \\
28.2 \\
29.0 \\
31.1\end{array}$ & $\begin{array}{l}282.5 \\
281.2 \\
277.6 \\
274.2 \\
281.6\end{array}$ & $\begin{array}{c}7.98 \\
9.81 \\
10.38 \\
7.03 \\
6.48\end{array}$ & $\begin{array}{l}21.84 \\
22.42 \\
24.85 \\
22.33 \\
21.67\end{array}$ & $\begin{array}{r}-2.51 \\
2.24 \\
-.71 \\
-3.11 \\
-3.89\end{array}$ & $\begin{array}{l}40.8 \\
33.1 \\
34.3 \\
36.0 \\
37.1\end{array}$ & $\begin{array}{l}72.9 \\
53.6 \\
72.1 \\
65.8 \\
62.8\end{array}$ & $\begin{array}{r}12.2 \\
14.3 \\
9.4 \\
11.6 \\
12.7\end{array}$ & $\begin{array}{r}1.13 \\
1.24 \\
.96 \\
.69 \\
.62\end{array}$ & $\begin{array}{r}2.11 \\
2.68 \\
2.12 \\
1.45 \\
.96\end{array}$ & $\begin{array}{l}.57 \\
.41 \\
.23 \\
.22 \\
.22\end{array}$ \\
\hline & $\begin{array}{l}21 \\
22 \\
23 \\
24 \\
25\end{array}$ & $\begin{array}{l}37.8 \\
31.3 \\
-2.2 \\
30.1 \\
26.5\end{array}$ & $\begin{array}{l}295.8 \\
281.2 \\
114.0 \\
299.7 \\
326.3\end{array}$ & $\begin{array}{r}7.45 \\
8.00 \\
9.38 \\
11.62 \\
5.60\end{array}$ & $\begin{array}{r}21.71 \\
21.17 \\
17.22 \\
18.01 \\
9.62\end{array}$ & $\begin{array}{r}-3.50 \\
.24 \\
-1.59 \\
2.54 \\
-.27\end{array}$ & $\begin{array}{l}36.7 \\
36.9 \\
45.7 \\
50.8 \\
80.4\end{array}$ & $\begin{array}{l}60.2 \\
57.8 \\
68.6 \\
85.0 \\
94.1\end{array}$ & $\begin{array}{l}13.2 \\
14.2 \\
23.9 \\
23.9 \\
49.4\end{array}$ & $\begin{array}{r}.58 \\
.60 \\
2.03 \\
2.42 \\
1.85\end{array}$ & $\begin{array}{l}1.19 \\
1.09 \\
4.56 \\
4.16 \\
3.27\end{array}$ & $\begin{array}{l}.35 \\
.26 \\
.36 \\
.34 \\
.45\end{array}$ \\
\hline & $\begin{array}{l}26 \\
27 \\
28 \\
29 \\
30\end{array}$ & $\begin{array}{l}63.8 \\
45.4 \\
32.3 \\
33.5 \\
36.1\end{array}$ & $\begin{array}{l}352.7 \\
292.4 \\
325.0 \\
329.0 \\
379.0\end{array}$ & $\begin{array}{l}3.66 \\
5.42 \\
6.28 \\
7.34 \\
7.49\end{array}$ & $\begin{array}{l}14.27 \\
12.71 \\
14.20 \\
16.42 \\
17.09\end{array}$ & $\begin{array}{r}-3.59 \\
-.82 \\
-.21 \\
1.46 \\
-3.33\end{array}$ & $\begin{array}{l}70.0 \\
54.6 \\
48.7 \\
41.5 \\
37.6\end{array}$ & $\begin{array}{l}95.2 \\
85.7 \\
67.8 \\
60.0 \\
71.3\end{array}$ & $\begin{array}{l}27.9 \\
27.6 \\
28.9 \\
20.7 \\
20.9\end{array}$ & $\begin{array}{l}1.34 \\
1.49 \\
2.10 \\
2.29 \\
2.31\end{array}$ & $\begin{array}{l}3.87 \\
2.75 \\
2.87 \\
4.12 \\
4.70\end{array}$ & $\begin{array}{l}.41 \\
.39 \\
.91 \\
.39 \\
.67\end{array}$ \\
\hline & $\begin{array}{l}31 \\
32 \\
33 \\
34 \\
35\end{array}$ & $\begin{array}{l}25.9 \\
11.8 \\
44.2 \\
42.2 \\
34.5\end{array}$ & $\begin{array}{l}332.2 \\
235.1 \\
318.3 \\
303.0 \\
215.4\end{array}$ & $\begin{array}{l}3.98 \\
2.90 \\
2.62 \\
5.83 \\
4.83\end{array}$ & $\begin{array}{r}9.68 \\
10.00 \\
11.75 \\
14.10 \\
8.10\end{array}$ & $\begin{array}{r}-0.05 \\
-2.13 \\
-8.72 \\
-3.86 \\
.91\end{array}$ & $\begin{array}{l}29.4 \\
30.6 \\
40.4 \\
39.5 \\
86.6\end{array}$ & $\begin{array}{l}40.3 \\
50.2 \\
77.3 \\
92.2 \\
94.8\end{array}$ & $\begin{array}{l}16.1 \\
14.8 \\
13.3 \\
14.0 \\
71.9\end{array}$ & $\begin{array}{r}2.44 \\
1.46 \\
.72 \\
.97 \\
.87\end{array}$ & $\begin{array}{l}4.01 \\
2.97 \\
1.28 \\
2.45 \\
2.01\end{array}$ & $\begin{array}{l}.83 \\
.47 \\
.22 \\
.26 \\
.26\end{array}$ \\
\hline & $\begin{array}{l}36 \\
37 \\
38 \\
39 \\
40\end{array}$ & $\begin{array}{l}58.1 \\
54.2 \\
35.6 \\
47.8 \\
54.2\end{array}$ & $\begin{array}{l}340.2 \\
322.4 \\
217.1 \\
391.5 \\
375.7\end{array}$ & $\begin{array}{l}5.75 \\
8.95 \\
9.63 \\
9.39 \\
7.14\end{array}$ & $\begin{array}{l}11.51 \\
14.18 \\
12.57 \\
15.18 \\
16.14\end{array}$ & $\begin{array}{r}-1.24 \\
4.54 \\
7.13 \\
4.34 \\
.11\end{array}$ & $\begin{array}{l}76.9 \\
68.2 \\
79.7 \\
67.6 \\
54.9\end{array}$ & $\begin{array}{l}96.3 \\
90.7 \\
91.6 \\
88.4 \\
86.5\end{array}$ & $\begin{array}{l}49.2 \\
45.4 \\
58.0 \\
42.4 \\
17.9\end{array}$ & $\begin{array}{r}.62 \\
.98 \\
2.53 \\
2.46 \\
1.40\end{array}$ & $\begin{array}{l}1.30 \\
2.43 \\
4.67 \\
4.79 \\
2.64\end{array}$ & $\begin{array}{l}.22 \\
.23 \\
.44 \\
.55 \\
.43\end{array}$ \\
\hline
\end{tabular}




\begin{tabular}{|c|c|c|c|c|c|c|c|c|c|c|c|}
\hline \multirow{2}{*}{$\begin{array}{l}\text { Calendar } \\
\text { day }\end{array}$} & \multicolumn{2}{|c|}{$\begin{array}{l}\text { Net radlation, In watts } \\
\text { per square meter }\end{array}$} & \multicolumn{3}{|c|}{$\begin{array}{c}\text { Air temperature at } 1.25 \text { meters, } \\
\text { in degrees Celsius }\end{array}$} & \multicolumn{3}{|c|}{$\begin{array}{l}\text { Humidity at } 1.25 \text { meters, } \\
\text { in degrees Celsius }\end{array}$} & \multicolumn{3}{|c|}{$\begin{array}{l}\text { Windspeed at } 1.25 \text { meters, } \\
\text { in meters per second }\end{array}$} \\
\hline & Mean & Maximum & Mean & Maximum & Minimum & Mean & Maximum & Minimum & Mean & Maximum & Minimum \\
\hline $\begin{array}{l}41 \\
42 \\
43 \\
44 \\
45\end{array}$ & $\begin{array}{l}56.7 \\
50.8 \\
46.7 \\
47.5 \\
62.1\end{array}$ & $\begin{array}{l}386.0 \\
395.6 \\
388.1 \\
330.0 \\
384.8\end{array}$ & $\begin{array}{l}9.15 \\
7.22 \\
4.11 \\
2.64 \\
5.59\end{array}$ & $\begin{array}{l}18.59 \\
11.89 \\
13.26 \\
14.48 \\
18.47\end{array}$ & $\begin{array}{r}-1.31 \\
-.30 \\
-4.31 \\
-7.94 \\
-4.00\end{array}$ & $\begin{array}{l}51.6 \\
35.2 \\
34.1 \\
51.2 \\
45.8\end{array}$ & $\begin{array}{l}91.2 \\
58.6 \\
68.9 \\
88.9 \\
79.8\end{array}$ & $\begin{array}{r}18.9 \\
20.6 \\
13.9 \\
15.5 \\
9.9\end{array}$ & $\begin{array}{r}2.43 \\
4.15 \\
1.78 \\
.83 \\
.75\end{array}$ & $\begin{array}{l}4.59 \\
7.24 \\
3.23 \\
1.99 \\
1.22\end{array}$ & $\begin{array}{r}0.41 \\
1.27 \\
.52 \\
.31 \\
.42\end{array}$ \\
\hline $\begin{array}{l}46 \\
47 \\
48 \\
49 \\
50\end{array}$ & $\begin{array}{l}38.3 \\
41.6 \\
21.0 \\
56.9 \\
84.3\end{array}$ & $\begin{array}{l}352.8 \\
259.0 \\
261.3 \\
474.9 \\
461.7\end{array}$ & $\begin{array}{l}4.57 \\
9.84 \\
1.11 \\
7.91 \\
6.48\end{array}$ & $\begin{array}{l}16.64 \\
17.68 \\
14.44 \\
13.69 \\
12.45\end{array}$ & $\begin{array}{r}-6.32 \\
1.32 \\
6.27 \\
4.15 \\
-.86\end{array}$ & $\begin{array}{l}49.0 \\
41.0 \\
49.6 \\
43.6 \\
51.6\end{array}$ & $\begin{array}{l}81.6 \\
69.9 \\
79.0 \\
59.7 \\
81.9\end{array}$ & $\begin{array}{l}16.8 \\
19.8 \\
29.6 \\
22.3 \\
32.2\end{array}$ & $\begin{array}{r}.60 \\
1.03 \\
3.59 \\
.16 \\
1.81\end{array}$ & $\begin{array}{l}1.11 \\
2.09 \\
6.16 \\
4.22 \\
3.18\end{array}$ & $\begin{array}{r}.27 \\
.30 \\
.63 \\
1.81 \\
.54\end{array}$ \\
\hline $\begin{array}{l}51 \\
52 \\
53 \\
54 \\
55\end{array}$ & $\begin{array}{l}43.2 \\
79.2 \\
90.4 \\
80.2 \\
80.4\end{array}$ & $\begin{array}{l}377.1 \\
476.7 \\
479.8 \\
450.7 \\
414.6\end{array}$ & $\begin{array}{l}7.42 \\
4.72 \\
5.37 \\
5.53 \\
6.83\end{array}$ & $\begin{array}{l}10.81 \\
13.95 \\
14.79 \\
15.14 \\
19.79\end{array}$ & $\begin{array}{r}.17 \\
-3.78 \\
-4.42 \\
-3.77 \\
-4.07\end{array}$ & $\begin{array}{l}55.4 \\
64.9 \\
51.0 \\
41.0 \\
44.6\end{array}$ & $\begin{array}{r}84.8 \\
92.6 \\
90.6 \\
68.7 \\
\cdot 76.9\end{array}$ & $\begin{array}{l}44.6 \\
32.2 \\
22.1 \\
19.0 \\
15.4\end{array}$ & $\begin{array}{r}2.58 \\
.92 \\
2.11 \\
1.00 \\
.74\end{array}$ & $\begin{array}{l}5.30 \\
1.88 \\
5.29 \\
1.51 \\
1.11\end{array}$ & $\begin{array}{l}.26 \\
.45 \\
.36 \\
.35 \\
.22\end{array}$ \\
\hline $\begin{array}{l}56 \\
57 \\
58 \\
59 \\
60\end{array}$ & $\begin{array}{l}79.4 \\
55.8 \\
73.5 \\
67.0 \\
76.6\end{array}$ & $\begin{array}{l}415.2 \\
380.0 \\
415.2 \\
429.1 \\
428.5\end{array}$ & $\begin{array}{l}9.66 \\
4.65 \\
2.66 \\
3.95 \\
4.76\end{array}$ & $\begin{array}{l}22.70 \\
21.87 \\
21.86 \\
21.94 \\
24.21\end{array}$ & $\begin{array}{r}-2.50 \\
6.16 \\
1.03 \\
3.52 \\
4.46\end{array}$ & $\begin{array}{l}39.7 \\
30.6 \\
44.4 \\
30.8 \\
33.5\end{array}$ & $\begin{array}{l}71.3 \\
45.7 \\
83.0 \\
61.6 \\
62.8\end{array}$ & $\begin{array}{l}13.6 \\
22.9 \\
24.0 \\
14.4 \\
16.7\end{array}$ & $\begin{array}{r}.95 \\
1.93 \\
1.43 \\
1.93 \\
2.04\end{array}$ & $\begin{array}{l}2.54 \\
4.71 \\
3.37 \\
3.16 \\
4.49\end{array}$ & $\begin{array}{l}.43 \\
.49 \\
.34 \\
.56 \\
.36\end{array}$ \\
\hline $\begin{array}{l}61 \\
62 \\
63 \\
64 \\
65\end{array}$ & $\begin{array}{l}85.4 \\
83.9 \\
83.5 \\
52.7 \\
49.7\end{array}$ & $\begin{array}{l}422.8 \\
421.3 \\
419.5 \\
378.2 \\
442.1\end{array}$ & $\begin{array}{l}12.89 \\
13.61 \\
13.97 \\
14.61 \\
15.56\end{array}$ & $\begin{array}{l}26.43 \\
26.99 \\
26.66 \\
22.37 \\
23.43\end{array}$ & $\begin{array}{r}.76 \\
2.33 \\
2.24 \\
3.52 \\
8.68\end{array}$ & $\begin{array}{l}43.5 \\
39.4 \\
38.0 \\
38.6 \\
44.6\end{array}$ & $\begin{array}{l}78.1 \\
66.6 \\
65.7 \\
67.8 \\
64.6\end{array}$ & $\begin{array}{l}15.8 \\
14.4 \\
14.3 \\
22.7 \\
25.3\end{array}$ & $\begin{array}{r}.67 \\
.80 \\
.83 \\
1.63 \\
1.54\end{array}$ & $\begin{array}{l}1.50 \\
1.27 \\
1.46 \\
3.26 \\
4.23\end{array}$ & $\begin{array}{l}.38 \\
.29 \\
.48 \\
.41 \\
.43\end{array}$ \\
\hline $\begin{array}{l}66 \\
67 \\
68 \\
69 \\
70\end{array}$ & $\begin{array}{l}66.0 \\
83.8 \\
84.1 \\
72.9 \\
31.6\end{array}$ & $\begin{array}{l}452.6 \\
438.6 \\
418.1 \\
406.7 \\
285.5\end{array}$ & $\begin{array}{l}15.71 \\
13.29 \\
12.48 \\
16.28 \\
13.97\end{array}$ & $\begin{array}{l}24.39 \\
25.03 \\
24.76 \\
24.99 \\
19.45\end{array}$ & $\begin{array}{r}7.79 \\
2.09 \\
.73 \\
4.72 \\
7.72\end{array}$ & $\begin{array}{l}43.2 \\
37.6 \\
32.7 \\
27.7 \\
35.8\end{array}$ & $\begin{array}{l}63.9 \\
76.8 \\
65.5 \\
51.8 \\
51.0\end{array}$ & $\begin{array}{r}19.6 \\
9.8 \\
8.9 \\
11.9 \\
23.0\end{array}$ & $\begin{array}{r}1.74 \\
1.19 \\
.81 \\
2.11 \\
2.67\end{array}$ & $\begin{array}{l}3.24 \\
2.40 \\
1.25 \\
4.34 \\
5.02\end{array}$ & $\begin{array}{l}.39 \\
.64 \\
.27 \\
.51 \\
.76\end{array}$ \\
\hline $\begin{array}{l}71 \\
72 \\
73 \\
74 \\
75\end{array}$ & $\begin{array}{l}89.4 \\
85.3 \\
95.0 \\
65.7 \\
46.1\end{array}$ & $\begin{array}{l}470.2 \\
463.6 \\
449.7 \\
425.0 \\
472.7\end{array}$ & $\begin{array}{l}16.44 \\
17.10 \\
15.52 \\
19.91 \\
22.07\end{array}$ & $\begin{array}{l}24.51 \\
27.83 \\
29.73 \\
30.40 \\
27.82\end{array}$ & $\begin{array}{r}8.81 \\
5.93 \\
1.44 \\
4.25 \\
18.76\end{array}$ & $\begin{array}{l}26.1 \\
20.0 \\
27.0 \\
20.3 \\
14.2\end{array}$ & $\begin{array}{l}40.4 \\
36.8 \\
56.4 \\
53.3 \\
19.6\end{array}$ & $\begin{array}{r}13.7 \\
6.0 \\
8.4 \\
8.6 \\
7.8\end{array}$ & $\begin{array}{r}2.96 \\
1.89 \\
.72 \\
1.80 \\
3.87\end{array}$ & $\begin{array}{l}4.00 \\
3.34 \\
1.22 \\
4.22 \\
5.15\end{array}$ & $\begin{array}{r}1.46 \\
.56 \\
.20 \\
.50 \\
2.07\end{array}$ \\
\hline $\begin{array}{l}76 \\
77 \\
78 \\
79 \\
80\end{array}$ & $\begin{array}{r}92.8 \\
102.4 \\
69.8 \\
117.8 \\
111.9\end{array}$ & $\begin{array}{l}456.0 \\
492.0 \\
480.4 \\
511.0 \\
515.7\end{array}$ & $\begin{array}{l}19.50 \\
19.75 \\
15.86 \\
13.63 \\
15.60\end{array}$ & $\begin{array}{l}26.19 \\
24.81 \\
20.27 \\
22.16 \\
23.37\end{array}$ & $\begin{array}{r}10.71 \\
15.00 \\
10.06 \\
6.58 \\
3.73\end{array}$ & $\begin{array}{l}20.1 \\
28.5 \\
50.8 \\
53.5 \\
44.0\end{array}$ & $\begin{array}{l}47.3 \\
50.5 \\
74.2 \\
91.2 \\
71.6\end{array}$ & $\begin{array}{r}9.3 \\
16.8 \\
40.5 \\
22.3 \\
29.0\end{array}$ & $\begin{array}{l}1.33 \\
4.04 \\
3.74 \\
1.01 \\
2.44\end{array}$ & $\begin{array}{l}3.45 \\
6.53 \\
6.24 \\
1.89 \\
4.66\end{array}$ & $\begin{array}{l}.34 \\
.94 \\
.67 \\
.20 \\
.47\end{array}$ \\
\hline
\end{tabular}


Table 6. Daily mean, maximum, and minimum micrometeorological data measured by micromet station at Rogers Spring Site 2, 1994-Continued

\begin{tabular}{|c|c|c|c|c|c|c|c|c|c|c|c|}
\hline \multirow{2}{*}{$\begin{array}{l}\text { Calendar } \\
\text { day }\end{array}$} & \multicolumn{2}{|c|}{$\begin{array}{l}\text { Net radiation, in watts } \\
\text { per square meter }\end{array}$} & \multicolumn{3}{|c|}{$\begin{array}{l}\text { Air temperature at } 1.25 \text { meters, } \\
\text { in degrees Celsius }\end{array}$} & \multicolumn{3}{|c|}{$\begin{array}{l}\text { Humidity at } 1.25 \text { meters, } \\
\text { in degrees Celsius }\end{array}$} & \multicolumn{3}{|c|}{$\begin{array}{l}\text { Windspeed at } 1.25 \text { meters, } \\
\text { in meters per second }\end{array}$} \\
\hline & Mean & Maximum & Mean & Maximum & Minimum & Mean & Maximum & Minimum & Mean & Maximum & Minimum \\
\hline $\begin{array}{l}81 \\
82 \\
83 \\
84 \\
85\end{array}$ & $\begin{array}{r}101.6 \\
107.1 \\
80.5 \\
116.0 \\
111.8\end{array}$ & $\begin{array}{l}522.4 \\
514.8 \\
445.3 \\
586.7 \\
522.5\end{array}$ & $\begin{array}{l}13.65 \\
10.44 \\
13.10 \\
9.90 \\
12.66\end{array}$ & $\begin{array}{l}18.21 \\
17.68 \\
18.98 \\
16.01 \\
22.36\end{array}$ & $\begin{array}{r}2.79 \\
.39 \\
8.95 \\
5.25 \\
1.03\end{array}$ & $\begin{array}{l}30.7 \\
24.6 \\
36.6 \\
57.7 \\
44.9\end{array}$ & $\begin{array}{l}48.9 \\
55.0 \\
52.1 \\
91.1 \\
85.4\end{array}$ & $\begin{array}{l}12.9 \\
11.0 \\
19.9 \\
25.3 \\
17.0\end{array}$ & $\begin{array}{l}4.42 \\
1.57 \\
1.92 \\
2.25 \\
1.74\end{array}$ & $\begin{array}{l}8.20 \\
2.49 \\
4.62 \\
4.07 \\
3.57\end{array}$ & $\begin{array}{r}0.60 \\
.50 \\
.54 \\
.65 \\
.50\end{array}$ \\
\hline $\begin{array}{l}86 \\
87 \\
88 \\
89 \\
90\end{array}$ & $\begin{array}{l}108.7 \\
100.4 \\
112.1 \\
111.1 \\
118.1\end{array}$ & $\begin{array}{l}505.6 \\
502.5 \\
483.3 \\
494.1 \\
521.4\end{array}$ & $\begin{array}{l}15.47 \\
16.17 \\
16.59 \\
18.73 \\
18.07\end{array}$ & $\begin{array}{l}25.94 \\
26.10 \\
27.45 \\
28.36 \\
28.69\end{array}$ & $\begin{array}{l}5.17 \\
1.92 \\
4.46 \\
8.35 \\
5.26\end{array}$ & $\begin{array}{l}24.8 \\
26.6 \\
28.4 \\
25.5 \\
27.5\end{array}$ & $\begin{array}{l}54.0 \\
60.3 \\
64.8 \\
54.8 \\
61.9\end{array}$ & $\begin{array}{r}7.0 \\
12.2 \\
10.7 \\
10.6 \\
11.2\end{array}$ & $\begin{array}{l}1.59 \\
1.75 \\
0.97 \\
1.08 \\
1.74\end{array}$ & $\begin{array}{l}3.09 \\
4.10 \\
1.69 \\
1.99 \\
4.06\end{array}$ & $\begin{array}{l}.56 \\
.48 \\
.20 \\
.48 \\
.43\end{array}$ \\
\hline $\begin{array}{l}91 \\
92 \\
93 \\
94 \\
95\end{array}$ & $\begin{array}{r}122.2 \\
105.2 \\
69.3 \\
118.0 \\
121.9\end{array}$ & $\begin{array}{l}530.4 \\
488.9 \\
443.6 \\
560.7 \\
539.0\end{array}$ & $\begin{array}{l}16.70 \\
18.42 \\
20.18 \\
16.01 \\
14.21\end{array}$ & $\begin{array}{l}27.07 \\
27.54 \\
25.67 \\
24.20 \\
23.11\end{array}$ & $\begin{array}{r}2.53 \\
6.17 \\
12.38 \\
5.22 \\
3.94\end{array}$ & $\begin{array}{l}30.2 \\
24.7 \\
26.5 \\
26.8 \\
22.7\end{array}$ & $\begin{array}{r}76.5 \\
58.7 \\
48.6 \\
67.1 \\
-40.3\end{array}$ & $\begin{array}{r}9.6 \\
10.2 \\
16.7 \\
6.5 \\
9.5\end{array}$ & $\begin{array}{l}2.09 \\
1.05 \\
3.30 \\
2.99 \\
1.19\end{array}$ & $\begin{array}{l}5.33 \\
2.30 \\
5.58 \\
6.05 \\
2.48\end{array}$ & $\begin{array}{r}.35 \\
.42 \\
1.02 \\
.69 \\
.44\end{array}$ \\
\hline $\begin{array}{r}96 \\
97 \\
98 \\
99 \\
100\end{array}$ & $\begin{array}{r}83.3 \\
119.2 \\
88.1 \\
93.7 \\
115.0\end{array}$ & $\begin{array}{l}536.2 \\
507.0 \\
499.9 \\
511.5 \\
564.2\end{array}$ & $\begin{array}{l}15.85 \\
16.21 \\
15.86 \\
12.67 \\
14.96\end{array}$ & $\begin{array}{l}24.01 \\
24.15 \\
21.33 \\
18.91 \\
22.82\end{array}$ & $\begin{array}{r}4.77 \\
3.13 \\
10.79 \\
6.50 \\
6.63\end{array}$ & $\begin{array}{l}25.5 \\
31.3 \\
34.0 \\
42.8 \\
30.8\end{array}$ & $\begin{array}{l}49.5 \\
78.1 \\
48.7 \\
60.0 \\
54.9\end{array}$ & $\begin{array}{l}15.2 \\
13.2 \\
23.4 \\
27.7 \\
12.6\end{array}$ & $\begin{array}{l}1.65 \\
1.15 \\
2.36 \\
1.57 \\
3.32\end{array}$ & $\begin{array}{l}3.34 \\
1.86 \\
4.14 \\
3.14 \\
5.14\end{array}$ & $\begin{array}{r}.28 \\
.39 \\
.89 \\
.56 \\
1.76\end{array}$ \\
\hline $\begin{array}{l}101 \\
102 \\
103 \\
104 \\
105\end{array}$ & $\begin{array}{l}127.9 \\
119.3 \\
125.9 \\
105.8 \\
123.2\end{array}$ & $\begin{array}{l}524.7 \\
477.1 \\
521.1 \\
464.4 \\
518.9\end{array}$ & $\begin{array}{l}15.79 \\
17.17 \\
18.96 \\
20.10 \\
22.37\end{array}$ & $\begin{array}{l}25.92 \\
27.96 \\
29.81 \\
31.15 \\
31.47\end{array}$ & $\begin{array}{r}4.95 \\
4.84 \\
4.58 \\
7.26 \\
12.43\end{array}$ & $\begin{array}{l}34.8 \\
30.0 \\
23.5 \\
29.1 \\
21.7\end{array}$ & $\begin{array}{l}61.3 \\
56.0 \\
58.2 \\
68.9 \\
49.5\end{array}$ & $\begin{array}{r}16.8 \\
13.2 \\
10.4 \\
9.8 \\
10.6\end{array}$ & $\begin{array}{r}1.28 \\
.96 \\
1.25 \\
1.38 \\
1.69\end{array}$ & $\begin{array}{l}2.80 \\
1.79 \\
2.34 \\
3.54 \\
3.64\end{array}$ & $\begin{array}{l}.24 \\
.46 \\
.35 \\
.33 \\
.20\end{array}$ \\
\hline $\begin{array}{l}106 \\
107 \\
108 \\
109 \\
110\end{array}$ & $\begin{array}{l}108.7 \\
106.7 \\
122.4 \\
134.2 \\
138.1\end{array}$ & $\begin{array}{l}455.3 \\
477.6 \\
529.1 \\
517.5 \\
531.6\end{array}$ & $\begin{array}{l}21.94 \\
23.59 \\
22.69 \\
22.82 \\
23.87\end{array}$ & $\begin{array}{l}33.09 \\
33.88 \\
34.22 \\
34.88 \\
35.29\end{array}$ & $\begin{array}{r}9.04 \\
13.36 \\
12.01 \\
9.80 \\
11.28\end{array}$ & $\begin{array}{l}24.8 \\
20.2 \\
24.6 \\
27.3 \\
24.5\end{array}$ & $\begin{array}{l}47.3 \\
39.6 \\
43.7 \\
51.8 \\
49.1\end{array}$ & $\begin{array}{r}9.4 \\
9.6 \\
10.1 \\
11.6 \\
9.2\end{array}$ & $\begin{array}{r}.88 \\
1.40 \\
.95 \\
.85 \\
1.30\end{array}$ & $\begin{array}{l}1.85 \\
3.76 \\
2.14 \\
1.98 \\
3.62\end{array}$ & $\begin{array}{l}.26 \\
.23 \\
.22 \\
.20 \\
.22\end{array}$ \\
\hline $\begin{array}{l}111 \\
112 \\
113 \\
114 \\
115\end{array}$ & $\begin{array}{l}129.7 \\
128.7 \\
110.2 \\
113.1 \\
110.6\end{array}$ & $\begin{array}{l}553.1 \\
545.8 \\
512.3 \\
529.6 \\
572.7\end{array}$ & $\begin{array}{l}24.87 \\
21.42 \\
18.26 \\
11.40 \\
12.37\end{array}$ & $\begin{array}{l}31.76 \\
27.61 \\
23.66 \\
16.87 \\
19.60\end{array}$ & $\begin{array}{r}17.51 \\
13.21 \\
11.64 \\
5.29 \\
6.23\end{array}$ & $\begin{array}{l}19.1 \\
21.6 \\
25.8 \\
43.9 \\
41.0\end{array}$ & $\begin{array}{l}28.8 \\
40.7 \\
44.5 \\
75.4 \\
58.5\end{array}$ & $\begin{array}{r}8.0 \\
12.2 \\
11.8 \\
25.1 \\
20.5\end{array}$ & $\begin{array}{l}3.13 \\
2.94 \\
5.70 \\
2.89 \\
3.25\end{array}$ & $\begin{array}{l}5.66 \\
5.36 \\
7.54 \\
5.80 \\
5.27\end{array}$ & $\begin{array}{l}.80 \\
.91 \\
.78 \\
.27 \\
.98\end{array}$ \\
\hline $\begin{array}{l}116 \\
117 \\
118 \\
119 \\
120\end{array}$ & $\begin{array}{l}106.3 \\
111.4 \\
130.1 \\
161.5 \\
110.5\end{array}$ & $\begin{array}{l}582.3 \\
605.4 \\
583.5 \\
590.4 \\
512.6\end{array}$ & $\begin{array}{l}10.28 \\
11.16 \\
12.68 \\
14.59 \\
17.63\end{array}$ & $\begin{array}{l}18.56 \\
17.28 \\
18.88 \\
24.27 \\
24.51\end{array}$ & $\begin{array}{l}3.93 \\
6.41 \\
6.92 \\
3.18 \\
9.77\end{array}$ & $\begin{array}{l}58.0 \\
74.6 \\
64.3 \\
48.6 \\
40.4\end{array}$ & $\begin{array}{l}91.5 \\
97.1 \\
96.0 \\
92.6 \\
72.7\end{array}$ & $\begin{array}{l}24.3 \\
44.3 \\
32.7 \\
17.0 \\
24.0\end{array}$ & $\begin{array}{r}1.36 \\
1.18 \\
1.13 \\
.93 \\
1.61\end{array}$ & $\begin{array}{l}3.97 \\
2.22 \\
3.44 \\
2.21 \\
3.67\end{array}$ & $\begin{array}{l}.32 \\
.26 \\
.28 \\
.33 \\
.37\end{array}$ \\
\hline
\end{tabular}




\begin{tabular}{|c|c|c|c|c|c|c|c|c|c|c|c|}
\hline \multirow{2}{*}{$\begin{array}{l}\text { Calendar } \\
\text { day }\end{array}$} & \multicolumn{2}{|c|}{$\begin{array}{l}\text { Net radiation, in watts } \\
\text { per square meter }\end{array}$} & \multicolumn{3}{|c|}{$\begin{array}{c}\text { Air temperature at } 1.25 \text { meters, } \\
\text { in degrees Celslus }\end{array}$} & \multicolumn{3}{|c|}{$\begin{array}{l}\text { Humidity at } 1.25 \text { meters, } \\
\text { in degrees Celsius }\end{array}$} & \multicolumn{3}{|c|}{$\begin{array}{l}\text { Windspeed at } 1.25 \text { meters, } \\
\text { In meters per second }\end{array}$} \\
\hline & Mean & Maximum & Mean & Maximum & Minimum & Mean & Maximum & Minimum & Mean & Maximum & Minimum \\
\hline $\begin{array}{l}121 \\
122 \\
123 \\
124 \\
125\end{array}$ & $\begin{array}{l}146.6 \\
141.2 \\
131.5 \\
157.1 \\
133.6\end{array}$ & $\begin{array}{l}566.9 \\
523.0 \\
557.8 \\
585.3 \\
561.8\end{array}$ & $\begin{array}{l}17.76 \\
19.75 \\
20.41 \\
23.63 \\
24.32\end{array}$ & $\begin{array}{l}27.51 \\
29.10 \\
29.40 \\
31.94 \\
30.67\end{array}$ & $\begin{array}{r}8.40 \\
7.52 \\
8.91 \\
11.74 \\
18.52\end{array}$ & $\begin{array}{l}41.3 \\
32.7 \\
29.8 \\
26.9 \\
21.7\end{array}$ & $\begin{array}{l}72.9 \\
71.8 \\
65.1 \\
44.5 \\
32.8\end{array}$ & $\begin{array}{l}16.5 \\
14.5 \\
13.9 \\
15.6 \\
12.4\end{array}$ & $\begin{array}{l}0.97 \\
1.24 \\
1.12 \\
2.25 \\
3.11\end{array}$ & $\begin{array}{l}2.23 \\
2.99 \\
1.94 \\
4.60 \\
4.30\end{array}$ & $\begin{array}{r}0.47 \\
.21 \\
.33 \\
.29 \\
.59\end{array}$ \\
\hline $\begin{array}{l}126 \\
127 \\
128 \\
129 \\
130\end{array}$ & $\begin{array}{r}162.5 \\
127.0 \\
93.8 \\
97.1 \\
145.7\end{array}$ & $\begin{array}{l}598.8 \\
564.7 \\
427.3 \\
424.7 \\
571.8\end{array}$ & $\begin{array}{l}17.85 \\
15.61 \\
16.76 \\
19.72 \\
22.49\end{array}$ & $\begin{array}{l}23.39 \\
21.14 \\
22.23 \\
29.43 \\
31.98\end{array}$ & $\begin{array}{r}12.67 \\
8.81 \\
11.06 \\
8.65 \\
8.94\end{array}$ & $\begin{array}{l}34.7 \\
43.4 \\
47.2 \\
40.9 \\
36.5\end{array}$ & $\begin{array}{l}52.7 \\
65.7 \\
75.0 \\
75.9 \\
73.7\end{array}$ & $\begin{array}{l}20.6 \\
27.0 \\
27.9 \\
18.4 \\
18.6\end{array}$ & $\begin{array}{r}3.36 \\
2.05 \\
1.97 \\
1.42 \\
.99\end{array}$ & $\begin{array}{l}5.14 \\
2.98 \\
3.23 \\
3.78 \\
1.92\end{array}$ & $\begin{array}{r}1.50 \\
.90 \\
.22 \\
.71 \\
.20\end{array}$ \\
\hline $\begin{array}{l}131 \\
132 \\
133 \\
134 \\
135\end{array}$ & $\begin{array}{l}144.1 \\
157.3 \\
160.6 \\
152.1 \\
141.6\end{array}$ & $\begin{array}{l}564.2 \\
555.0 \\
560.3 \\
557.4 \\
595.8\end{array}$ & $\begin{array}{l}26.51 \\
25.53 \\
24.77 \\
26.03 \\
26.46\end{array}$ & $\begin{array}{l}35.03 \\
33.24 \\
34.31 \\
34.98 \\
31.84\end{array}$ & $\begin{array}{l}16.07 \\
12.47 \\
12.80 \\
11.44 \\
21.15\end{array}$ & $\begin{array}{l}32.3 \\
35.6 \\
42.6 \\
29.6 \\
17.2\end{array}$ & $\begin{array}{l}60.1 \\
68.0 \\
87.0 \\
69.9 \\
26.3\end{array}$ & $\begin{array}{r}17.3 \\
22.4 \\
19.8 \\
13.7 \\
7.5\end{array}$ & $\begin{array}{r}1.30 \\
1.82 \\
.71 \\
1.96 \\
5.42\end{array}$ & $\begin{array}{l}3.45 \\
4.18 \\
1.53 \\
4.42 \\
7.17\end{array}$ & $\begin{array}{r}.20 \\
.22 \\
.23 \\
.20 \\
2.51\end{array}$ \\
\hline $\begin{array}{l}136 \\
137 \\
138 \\
139 \\
140\end{array}$ & $\begin{array}{l}156.6 \\
143.0 \\
161.5 \\
151.4 \\
156.7\end{array}$ & $\begin{array}{l}597.9 \\
589.4 \\
592.9 \\
558.8 \\
566.3\end{array}$ & $\begin{array}{l}19.08 \\
15.87 \\
15.04 \\
15.03 \\
16.24\end{array}$ & $\begin{array}{l}24.70 \\
21.61 \\
21.13 \\
23.40 \\
26.53\end{array}$ & $\begin{array}{r}9.61 \\
10.42 \\
8.70 \\
7.52 \\
4.35\end{array}$ & $\begin{array}{l}33.1 \\
33.9 \\
39.4 \\
44.6 \\
36.7\end{array}$ & $\begin{array}{l}60.4 \\
49.1 \\
57.7 \\
71.1 \\
72.7\end{array}$ & $\begin{array}{l}14.5 \\
22.7 \\
21.7 \\
20.8 \\
12.6\end{array}$ & $\begin{array}{r}4.62 \\
3.29 \\
2.89 \\
1.31 \\
.75\end{array}$ & $\begin{array}{l}6.54 \\
4.44 \\
4.26 \\
2.34 \\
1.68\end{array}$ & $\begin{array}{r}.59 \\
1.59 \\
1.07 \\
.29 \\
.21\end{array}$ \\
\hline $\begin{array}{l}141 \\
142 \\
143 \\
144 \\
145\end{array}$ & $\begin{array}{l}156.7 \\
158.1 \\
162.5 \\
127.9 \\
140.1\end{array}$ & $\begin{array}{l}564.1 \\
567.0 \\
573.7 \\
521.1 \\
610.2\end{array}$ & $\begin{array}{l}18.84 \\
21.00 \\
22.93 \\
24.70 \\
26.07\end{array}$ & $\begin{array}{l}30.14 \\
31.99 \\
34.22 \\
34.54 \\
33.32\end{array}$ & $\begin{array}{r}4.56 \\
7.12 \\
9.84 \\
11.16 \\
17.46\end{array}$ & $\begin{array}{l}22.7 \\
22.1 \\
28.0 \\
25.3 \\
34.4\end{array}$ & $\begin{array}{l}49.2 \\
46.9 \\
57.8 \\
49.3 \\
58.3\end{array}$ & $\begin{array}{r}8.2 \\
9.0 \\
10.9 \\
10.2 \\
18.8\end{array}$ & $\begin{array}{r}1.12 \\
1.20 \\
.73 \\
1.10 \\
1.95\end{array}$ & $\begin{array}{l}2.81 \\
2.87 \\
1.92 \\
2.98 \\
3.25\end{array}$ & $\begin{array}{l}.23 \\
.21 \\
.27 \\
.20 \\
.25\end{array}$ \\
\hline $\begin{array}{l}146 \\
147 \\
148 \\
149 \\
150\end{array}$ & $\begin{array}{l}158.6 \\
105.9 \\
157.8 \\
158.8 \\
109.1\end{array}$ & $\begin{array}{l}568.6 \\
497.9 \\
560.6 \\
564.6 \\
597.4\end{array}$ & $\begin{array}{l}25.79 \\
26.70 \\
26.43 \\
26.13 \\
26.92\end{array}$ & $\begin{array}{l}32.62 \\
31.66 \\
34.24 \\
36.02 \\
35.57\end{array}$ & $\begin{array}{l}14.42 \\
20.42 \\
17.73 \\
11.06 \\
15.82\end{array}$ & $\begin{array}{l}39.2 \\
21.1 \\
19.6 \\
17.1 \\
26.3\end{array}$ & $\begin{array}{l}75.3 \\
30.7 \\
37.4 \\
55.4 \\
78.0\end{array}$ & $\begin{array}{r}23.0 \\
13.5 \\
9.6 \\
5.0 \\
9.0\end{array}$ & $\begin{array}{l}1.98 \\
3.10 \\
1.35 \\
1.48 \\
1.76\end{array}$ & $\begin{array}{l}3.86 \\
4.42 \\
2.02 \\
2.99 \\
3.62\end{array}$ & $\begin{array}{r}.24 \\
1.20 \\
.25 \\
.26 \\
.29\end{array}$ \\
\hline $\begin{array}{l}151 \\
152 \\
153 \\
154 \\
155\end{array}$ & $\begin{array}{l}175.8 \\
168.2 \\
163.4 \\
129.4 \\
157.3\end{array}$ & $\begin{array}{l}578.1 \\
568.2 \\
574.4 \\
481.3 \\
568.2\end{array}$ & $\begin{array}{l}23.22 \\
24.74 \\
27.10 \\
26.97 \\
25.02\end{array}$ & $\begin{array}{l}32.21 \\
35.81 \\
37.14 \\
35.17 \\
34.55\end{array}$ & $\begin{array}{r}15.39 \\
12.47 \\
12.32 \\
13.55 \\
9.93\end{array}$ & $\begin{array}{l}50.3 \\
36.9 \\
24.6 \\
18.4 \\
20.4\end{array}$ & $\begin{array}{l}88.5 \\
65.8 \\
62.7 \\
50.0 \\
53.7\end{array}$ & $\begin{array}{r}20.4 \\
13.0 \\
11.9 \\
4.1 \\
10.2\end{array}$ & $\begin{array}{r}.90 \\
.51 \\
1.13 \\
2.41 \\
1.03\end{array}$ & $\begin{array}{l}1.75 \\
1.30 \\
2.78 \\
3.65 \\
1.65\end{array}$ & $\begin{array}{l}.20 \\
.20 \\
.20 \\
.46 \\
.40\end{array}$ \\
\hline $\begin{array}{l}156 \\
157 \\
158 \\
159 \\
160\end{array}$ & $\begin{array}{l}166.3 \\
137.7 \\
161.8 \\
163.4 \\
159.8\end{array}$ & $\begin{array}{l}592.2 \\
526.2 \\
561.4 \\
558.7 \\
558.8\end{array}$ & $\begin{array}{l}26.60 \\
24.52 \\
22.45 \\
25.19 \\
25.64\end{array}$ & $\begin{array}{l}34.33 \\
31.58 \\
32.72 \\
35.46 \\
39.02\end{array}$ & $\begin{array}{r}15.74 \\
18.68 \\
9.29 \\
14.54 \\
9.72\end{array}$ & $\begin{array}{l}20.3 \\
25.6 \\
28.8 \\
20.5 \\
25.0\end{array}$ & $\begin{array}{l}37.0 \\
46.7 \\
65.4 \\
59.2 \\
65.6\end{array}$ & $\begin{array}{l}9.6 \\
9.8 \\
9.3 \\
8.3 \\
6.1\end{array}$ & $\begin{array}{r}2.38 \\
3.39 \\
.85 \\
.99 \\
.45\end{array}$ & $\begin{array}{l}4.24 \\
5.19 \\
1.82 \\
2.91 \\
1.01\end{array}$ & $\begin{array}{r}.54 \\
1.00 \\
.20 \\
.20 \\
.20\end{array}$ \\
\hline
\end{tabular}


Table 6. Daily mean, maximum, and minimum micrometeorological data measured by micromet station at Rogers Spring Site 2, 1994—Continued

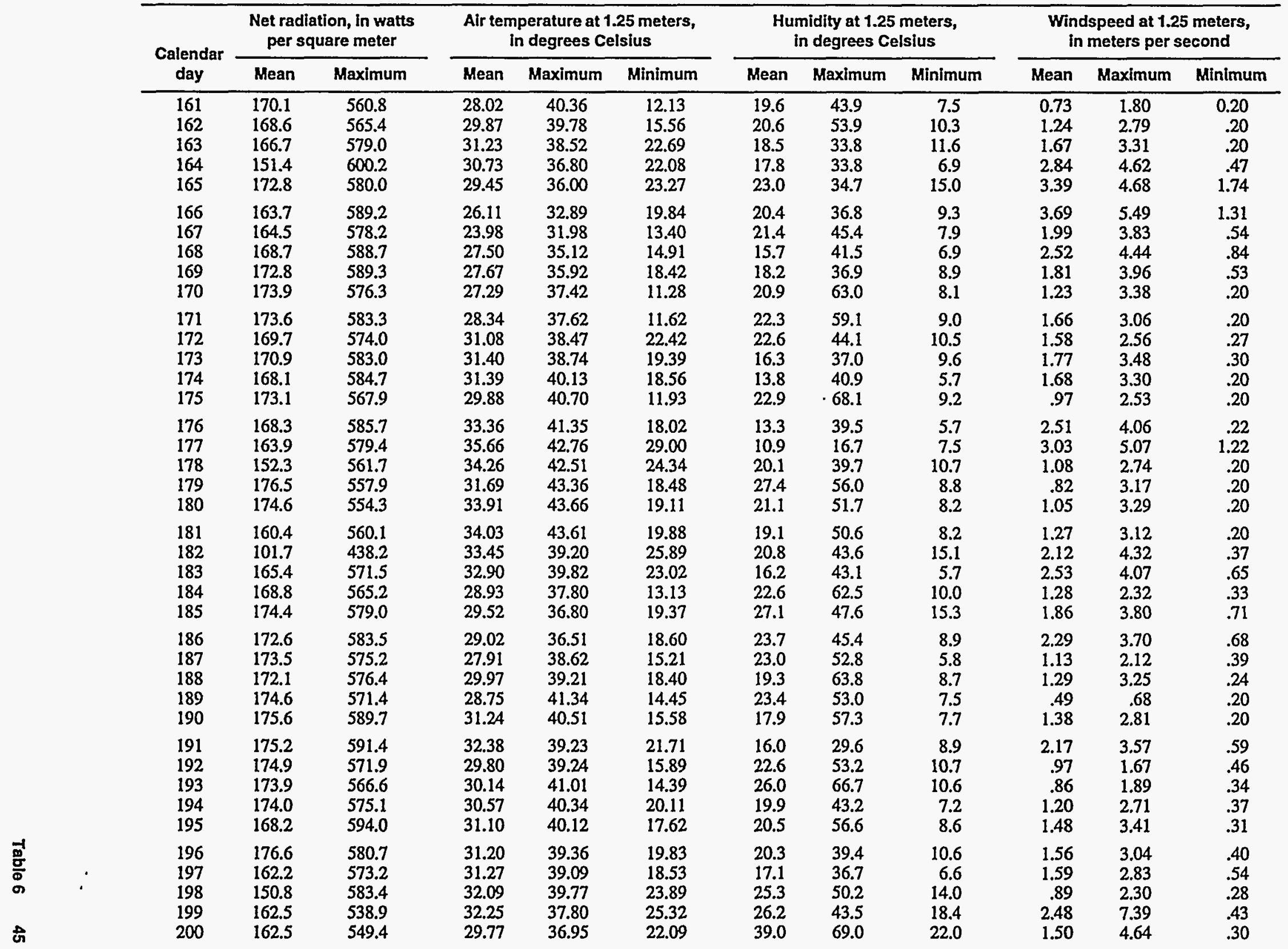


Table 6. Daily mean, maximum, and minimum micrometeorological data measured by micromet station at Rogers Spring Site 2, 1994-Continued

政

\begin{tabular}{|c|c|c|c|c|c|c|c|c|c|c|c|}
\hline \multirow{2}{*}{$\begin{array}{l}\text { Calendar } \\
\text { day }\end{array}$} & \multicolumn{2}{|c|}{$\begin{array}{l}\text { Net radiation, In watts } \\
\text { per square meter }\end{array}$} & \multicolumn{3}{|c|}{$\begin{array}{l}\text { Alr temperature at } 1.25 \text { meters, } \\
\text { in degrees Celsius }\end{array}$} & \multicolumn{3}{|c|}{$\begin{array}{l}\text { Humidity at } 1.25 \text { meters, } \\
\text { in degrees Celsius }\end{array}$} & \multicolumn{3}{|c|}{$\begin{array}{l}\text { Windspeed at } 1.25 \text { meters, } \\
\text { in meters per second }\end{array}$} \\
\hline & Mean & Maximum & Mean & Maximum & Minimum & Mean & Maximum & Minimum & Mean & Maximum & Minimum \\
\hline $\begin{array}{l}201 \\
202 \\
203 \\
204 \\
205\end{array}$ & $\begin{array}{l}156.5 \\
167.5 \\
182.3 \\
186.3 \\
178.0\end{array}$ & $\begin{array}{l}531.2 \\
558.9 \\
593.2 \\
597.0 \\
590.7\end{array}$ & $\begin{array}{l}29.06 \\
30.25 \\
31.67 \\
31.23 \\
31.78\end{array}$ & $\begin{array}{l}37.42 \\
37.65 \\
38.73 \\
39.17 \\
39.32\end{array}$ & $\begin{array}{l}20.03 \\
19.21 \\
23.29 \\
19.21 \\
22.97\end{array}$ & $\begin{array}{l}44.8 \\
36.1 \\
25.2 \\
26.7 \\
20.3\end{array}$ & $\begin{array}{l}83.1 \\
71.1 \\
40.4 \\
63.7 \\
40.1\end{array}$ & $\begin{array}{r}22.8 \\
20.9 \\
15.0 \\
14.2 \\
9.6\end{array}$ & $\begin{array}{l}1.20 \\
1.26 \\
1.43 \\
1.31 \\
1.39\end{array}$ & $\begin{array}{l}2.55 \\
3.01 \\
2.62 \\
3.28 \\
2.40\end{array}$ & $\begin{array}{r}0.61 \\
.20 \\
.65 \\
.35 \\
.47\end{array}$ \\
\hline $\begin{array}{l}206 \\
207 \\
208 \\
209 \\
210\end{array}$ & $\begin{array}{l}173.1 \\
167.2 \\
178.6 \\
175.9 \\
153.9\end{array}$ & $\begin{array}{l}582.8 \\
588.5 \\
584.4 \\
584.3 \\
549.1\end{array}$ & $\begin{array}{l}31.02 \\
29.99 \\
31.44 \\
32.36 \\
33.09\end{array}$ & $\begin{array}{l}40.85 \\
41.53 \\
41.64 \\
41.66 \\
39.96\end{array}$ & $\begin{array}{l}16.83 \\
15.08 \\
15.61 \\
20.33 \\
22.36\end{array}$ & $\begin{array}{l}18.1 \\
23.9 \\
23.2 \\
23.1 \\
27.2\end{array}$ & $\begin{array}{l}49.6 \\
58.9 \\
64.9 \\
48.0 \\
47.8\end{array}$ & $\begin{array}{r}8.3 \\
9.4 \\
10.0 \\
12.1 \\
18.3\end{array}$ & $\begin{array}{r}.99 \\
.78 \\
1.01 \\
1.05 \\
1.62\end{array}$ & $\begin{array}{l}2.07 \\
1.80 \\
2.30 \\
2.94 \\
2.94\end{array}$ & $\begin{array}{l}.50 \\
.31 \\
.37 \\
.20 \\
.41\end{array}$ \\
\hline $\begin{array}{l}211 \\
212 \\
213 \\
214 \\
215\end{array}$ & $\begin{array}{l}174.7 \\
174.1 \\
173.1 \\
169.6 \\
173.7\end{array}$ & $\begin{array}{l}578.7 \\
586.3 \\
587.1 \\
585.4 \\
572.3\end{array}$ & $\begin{array}{l}33.32 \\
32.10 \\
32.74 \\
31.67 \\
28.63\end{array}$ & $\begin{array}{l}39.31 \\
39.34 \\
39.69 \\
39.60 \\
40.13\end{array}$ & $\begin{array}{l}27.81 \\
23.17 \\
22.51 \\
19.68 \\
13.05\end{array}$ & $\begin{array}{l}24.4 \\
20.0 \\
16.7 \\
14.4 \\
20.9\end{array}$ & $\begin{array}{l}38.8 \\
34.7 \\
32.4 \\
29.4 \\
62.7\end{array}$ & $\begin{array}{r}16.1 \\
11.3 \\
10.2 \\
7.1 \\
5.6\end{array}$ & $\begin{array}{l}2.02 \\
1.71 \\
1.94 \\
1.47 \\
1.02\end{array}$ & $\begin{array}{l}3.49 \\
3.25 \\
3.20 \\
2.62 \\
3.24\end{array}$ & $\begin{array}{l}.80 \\
.60 \\
.67 \\
.68 \\
.25\end{array}$ \\
\hline $\begin{array}{l}216 \\
217 \\
218 \\
219 \\
220\end{array}$ & $\begin{array}{l}173.1 \\
168.0 \\
168.7 \\
161.3 \\
115.3\end{array}$ & $\begin{array}{l}580.6 \\
589.7 \\
567.7 \\
571.3 \\
493.9\end{array}$ & $\begin{array}{l}28.04 \\
31.38 \\
34.07 \\
33.27 \\
32.62\end{array}$ & $\begin{array}{l}39.97 \\
41.64 \\
43.10 \\
41.37 \\
36.75\end{array}$ & $\begin{array}{l}12.01 \\
15.64 \\
19.68 \\
18.25 \\
28.77\end{array}$ & $\begin{array}{l}17.6 \\
17.6 \\
19.1 \\
22.8 \\
23.7\end{array}$ & $\begin{array}{r}-49.0 \\
41.2 \\
46.9 \\
60.5 \\
34.6\end{array}$ & $\begin{array}{r}5.9 \\
8.4 \\
10.7 \\
11.8 \\
18.7\end{array}$ & $\begin{array}{l}1.20 \\
1.87 \\
1.05 \\
1.72 \\
3.45\end{array}$ & $\begin{array}{l}2.93 \\
4.28 \\
2.40 \\
3.95 \\
5.43\end{array}$ & $\begin{array}{r}.44 \\
.45 \\
.32 \\
.20 \\
1.58\end{array}$ \\
\hline $\begin{array}{l}221 \\
222 \\
223 \\
224 \\
225\end{array}$ & $\begin{array}{l}152.9 \\
151.4 \\
172.2 \\
156.6 \\
157.8\end{array}$ & $\begin{array}{l}576.6 \\
586.2 \\
575.5 \\
568.8 \\
561.4\end{array}$ & $\begin{array}{l}31.91 \\
31.42 \\
32.65 \\
33.52 \\
32.07\end{array}$ & $\begin{array}{l}37.61 \\
38.48 \\
40.56 \\
39.08 \\
40.00\end{array}$ & $\begin{array}{l}27.17 \\
23.34 \\
24.32 \\
27.39 \\
26.27\end{array}$ & $\begin{array}{l}34.4 \\
38.3 \\
31.9 \\
28.9 \\
35.7\end{array}$ & $\begin{array}{l}44.8 \\
65.4 \\
49.4 \\
46.9 \\
47.3\end{array}$ & $\begin{array}{l}24.7 \\
21.3 \\
19.2 \\
21.4 \\
22.4\end{array}$ & $\begin{array}{l}2.83 \\
1.19 \\
1.00 \\
2.22 \\
1.29\end{array}$ & $\begin{array}{l}3.92 \\
2.69 \\
2.47 \\
4.41 \\
3.31\end{array}$ & $\begin{array}{r}1.68 \\
.50 \\
.22 \\
.65 \\
.47\end{array}$ \\
\hline $\begin{array}{l}226 \\
227 \\
228 \\
229 \\
230\end{array}$ & $\begin{array}{l}171.9 \\
171.6 \\
166.3 \\
142.8 \\
148.9\end{array}$ & $\begin{array}{l}557.6 \\
555.2 \\
566.7 \\
580.0 \\
544.6\end{array}$ & $\begin{array}{l}30.78 \\
31.28 \\
34.11 \\
31.31 \\
32.20\end{array}$ & $\begin{array}{l}40.97 \\
42.43 \\
42.18 \\
37.71 \\
37.72\end{array}$ & $\begin{array}{l}20.14 \\
18.41 \\
22.19 \\
22.98 \\
25.77\end{array}$ & $\begin{array}{l}40.3 \\
34.8 \\
23.8 \\
31.5 \\
31.3\end{array}$ & $\begin{array}{l}72.4 \\
72.3 \\
44.9 \\
45.3 \\
50.1\end{array}$ & $\begin{array}{l}18.4 \\
14.0 \\
12.7 \\
21.5 \\
19.8\end{array}$ & $\begin{array}{r}.53 \\
.53 \\
1.79 \\
2.59 \\
1.94\end{array}$ & $\begin{array}{r}.79 \\
.77 \\
3.51 \\
5.66 \\
3.15\end{array}$ & $\begin{array}{l}.20 \\
.20 \\
.37 \\
.61 \\
.46\end{array}$ \\
\hline $\begin{array}{l}231 \\
232 \\
233 \\
234 \\
235\end{array}$ & $\begin{array}{l}159.8 \\
161.3 \\
155.7 \\
151.8 \\
158.2\end{array}$ & $\begin{array}{l}548.1 \\
557.9 \\
564.6 \\
560.9 \\
551.1\end{array}$ & $\begin{array}{l}31.75 \\
31.55 \\
30.89 \\
28.74 \\
25.22\end{array}$ & $\begin{array}{l}38.40 \\
39.11 \\
37.48 \\
35.66 \\
36.99\end{array}$ & $\begin{array}{l}22.28 \\
19.78 \\
22.68 \\
22.00 \\
12.59\end{array}$ & $\begin{array}{l}32.9 \\
30.5 \\
15.5 \\
15.1 \\
17.2\end{array}$ & $\begin{array}{l}58.0 \\
66.5 \\
30.2 \\
27.9 \\
36.8\end{array}$ & $\begin{array}{r}21.3 \\
16.9 \\
5.0 \\
5.4 \\
5.7\end{array}$ & $\begin{array}{l}1.59 \\
1.64 \\
3.09 \\
2.40 \\
1.35\end{array}$ & $\begin{array}{l}3.26 \\
3.56 \\
4.85 \\
3.71 \\
2.97\end{array}$ & $\begin{array}{l}.38 \\
.21 \\
.94 \\
.71 \\
.36\end{array}$ \\
\hline $\begin{array}{l}236 \\
237 \\
238 \\
239 \\
240\end{array}$ & $\begin{array}{l}159.2 \\
154.9 \\
145.8 \\
135.8 \\
154.7\end{array}$ & $\begin{array}{l}552.2 \\
559.8 \\
546.4 \\
535.4 \\
545.6\end{array}$ & $\begin{array}{l}27.31 \\
31.17 \\
30.52 \\
29.94 \\
30.79\end{array}$ & $\begin{array}{l}39.04 \\
38.95 \\
38.72 \\
37.04 \\
38.58\end{array}$ & $\begin{array}{l}12.15 \\
20.58 \\
19.03 \\
24.67 \\
19.90\end{array}$ & $\begin{array}{l}18.1 \\
12.6 \\
20.8 \\
34.4 \\
21.7\end{array}$ & $\begin{array}{l}45.7 \\
30.3 \\
35.9 \\
61.6 \\
42.1\end{array}$ & $\begin{array}{r}6.0 \\
7.5 \\
9.2 \\
16.2 \\
12.5\end{array}$ & $\begin{array}{l}1.46 \\
2.24 \\
2.19 \\
1.75 \\
1.81\end{array}$ & $\begin{array}{l}3.07 \\
4.26 \\
4.26 \\
3.15 \\
3.44\end{array}$ & $\begin{array}{l}.47 \\
.38 \\
.52 \\
.70 \\
.68\end{array}$ \\
\hline
\end{tabular}


Table 6. Daily mean, maximum, and minimum micrometeorological data measured by micromet station at Rogers Spring Site 2, 1994—Continued

\begin{tabular}{|c|c|c|c|c|c|c|c|c|c|c|c|}
\hline \multirow{2}{*}{$\begin{array}{l}\text { Calendar } \\
\text { day }\end{array}$} & \multicolumn{2}{|c|}{$\begin{array}{l}\text { Net radiation, in watts } \\
\text { per square meter }\end{array}$} & \multicolumn{3}{|c|}{$\begin{array}{c}\text { Air temperature at } 1.25 \text { meters, } \\
\text { in degrees Celsius }\end{array}$} & \multicolumn{3}{|c|}{$\begin{array}{l}\text { Humidity at } 1.25 \text { meters, } \\
\text { in degrees Celsius }\end{array}$} & \multicolumn{3}{|c|}{$\begin{array}{l}\text { Windspeed at } 1.25 \text { meters, } \\
\text { in meters per second }\end{array}$} \\
\hline & Mean & Maximum & Mean & Maximum & Minimum & Mean & Maximum & Minimum & Mean & Maximum & Minimum \\
\hline $\begin{array}{l}241 \\
242 \\
243 \\
244 \\
245\end{array}$ & $\begin{array}{l}152.5 \\
152.9 \\
137.6 \\
147.5 \\
134.0\end{array}$ & $\begin{array}{l}543.9 \\
538.6 \\
546.0 \\
539.8 \\
511.5\end{array}$ & $\begin{array}{l}28.16 \\
24.42 \\
26.22 \\
27.76 \\
27.85\end{array}$ & $\begin{array}{l}37.66 \\
37.19 \\
36.16 \\
35.59 \\
35.16\end{array}$ & $\begin{array}{r}14.40 \\
9.49 \\
11.24 \\
18.29 \\
16.34\end{array}$ & $\begin{array}{l}18.2 \\
23.4 \\
18.5 \\
16.8 \\
14.5\end{array}$ & $\begin{array}{l}48.3 \\
64.6 \\
53.2 \\
32.7 \\
31.9\end{array}$ & $\begin{array}{l}5.9 \\
5.8 \\
7.1 \\
8.1 \\
4.9\end{array}$ & $\begin{array}{l}1.50 \\
1.18 \\
1.74 \\
2.37 \\
3.02\end{array}$ & $\begin{array}{l}2.50 \\
3.04 \\
3.65 \\
3.97 \\
5.12\end{array}$ & $\begin{array}{r}0.41 \\
.41 \\
.36 \\
.66 \\
.60\end{array}$ \\
\hline $\begin{array}{l}246 \\
247 \\
248 \\
249 \\
255\end{array}$ & $\begin{array}{r}146.1 \\
149.2 \\
150.6 \\
-9.0 \\
190.3\end{array}$ & $\begin{array}{r}527.8 \\
518.6 \\
519.5 \\
-7.9 \\
525.7\end{array}$ & $\begin{array}{l}23.88 \\
24.04 \\
26.36 \\
20.21 \\
23.22\end{array}$ & $\begin{array}{l}34.16 \\
36.05 \\
37.35 \\
21.37 \\
27.44\end{array}$ & $\begin{array}{r}11.68 \\
9.95 \\
14.09 \\
17.70 \\
16.48\end{array}$ & $\begin{array}{l}22.2 \\
31.6 \\
38.0 \\
51.3 \\
23.0\end{array}$ & $\begin{array}{l}48.1 \\
61.7 \\
68.7 \\
62.9 \\
50.7\end{array}$ & $\begin{array}{r}9.5 \\
14.9 \\
18.5 \\
43.3 \\
11.8\end{array}$ & $\begin{array}{r}1.28 \\
.91 \\
.93 \\
.53 \\
3.76\end{array}$ & $\begin{array}{r}2.72 \\
2.02 \\
2.19 \\
.65 \\
5.67\end{array}$ & $\begin{array}{r}.46 \\
.30 \\
.30 \\
.43 \\
1.63\end{array}$ \\
\hline $\begin{array}{l}256 \\
257 \\
258 \\
259 \\
260\end{array}$ & $\begin{array}{l}131.2 \\
132.7 \\
134.5 \\
112.8 \\
109.4\end{array}$ & $\begin{array}{l}497.0 \\
493.1 \\
494.5 \\
489.8 \\
437.1\end{array}$ & $\begin{array}{l}17.34 \\
19.54 \\
21.82 \\
21.80 \\
22.20\end{array}$ & $\begin{array}{l}27.17 \\
31.08 \\
33.99 \\
33.84 \\
34.25\end{array}$ & $\begin{array}{r}7.90 \\
9.18 \\
8.33 \\
7.81 \\
10.93\end{array}$ & $\begin{array}{l}39.7 \\
33.6 \\
26.6 \\
30.6 \\
24.5\end{array}$ & $\begin{array}{r}63.2 \\
59.6 \\
53.6 \\
60.6 \\
.46 .5\end{array}$ & $\begin{array}{r}16.7 \\
13.4 \\
9.8 \\
10.7 \\
10.9\end{array}$ & $\begin{array}{r}1.17 \\
.94 \\
1.05 \\
.88 \\
1.11\end{array}$ & $\begin{array}{l}2.02 \\
1.70 \\
3.09 \\
2.13 \\
3.58\end{array}$ & $\begin{array}{l}.41 \\
.38 \\
.32 \\
.31 \\
.37\end{array}$ \\
\hline $\begin{array}{l}261 \\
262 \\
263 \\
264 \\
265\end{array}$ & $\begin{array}{r}104.1 \\
89.5 \\
122.9 \\
129.4 \\
123.2\end{array}$ & $\begin{array}{l}435.5 \\
495.8 \\
519.6 \\
480.1 \\
472.0\end{array}$ & $\begin{array}{l}22.69 \\
22.41 \\
24.46 \\
23.74 \\
22.80\end{array}$ & $\begin{array}{l}35.07 \\
30.78 \\
33.02 \\
34.34 \\
35.44\end{array}$ & $\begin{array}{r}11.81 \\
13.55 \\
15.02 \\
13.53 \\
9.61\end{array}$ & $\begin{array}{l}32.4 \\
40.1 \\
37.9 \\
35.4 \\
33.2\end{array}$ & $\begin{array}{l}58.3 \\
62.8 \\
62.8 \\
61.6 \\
68.1\end{array}$ & $\begin{array}{l}13.9 \\
25.0 \\
22.7 \\
13.2 \\
13.7\end{array}$ & $\begin{array}{r}.70 \\
1.69 \\
1.53 \\
.98 \\
.74\end{array}$ & $\begin{array}{l}1.35 \\
3.06 \\
2.45 \\
2.31 \\
1.38\end{array}$ & $\begin{array}{l}.36 \\
.45 \\
.56 \\
.30 \\
.41\end{array}$ \\
\hline $\begin{array}{l}266 \\
267 \\
268 \\
269 \\
270\end{array}$ & $\begin{array}{r}125.7 \\
98.7 \\
124.9 \\
117.7 \\
113.6\end{array}$ & $\begin{array}{l}466.4 \\
442.8 \\
467.3 \\
460.6 \\
453.2\end{array}$ & $\begin{array}{l}23.21 \\
25.16 \\
22.03 \\
22.11 \\
23.94\end{array}$ & $\begin{array}{l}35.79 \\
32.79 \\
34.33 \\
35.96 \\
35.63\end{array}$ & $\begin{array}{r}10.55 \\
17.36 \\
11.18 \\
9.24 \\
9.14\end{array}$ & $\begin{array}{l}27.9 \\
35.5 \\
35.8 \\
29.9 \\
31.0\end{array}$ & $\begin{array}{l}46.0 \\
57.0 \\
64.8 \\
55.2 \\
64.4\end{array}$ & $\begin{array}{l}12.0 \\
20.7 \\
14.4 \\
12.9 \\
14.0\end{array}$ & $\begin{array}{r}.79 \\
1.27 \\
.96 \\
.74 \\
1.27\end{array}$ & $\begin{array}{l}1.38 \\
2.95 \\
2.25 \\
1.25 \\
3.41\end{array}$ & $\begin{array}{l}.27 \\
.37 \\
.36 \\
.39 \\
.37\end{array}$ \\
\hline $\begin{array}{l}271 \\
272 \\
273 \\
274 \\
275\end{array}$ & $\begin{array}{l}73.7 \\
57.2 \\
86.2 \\
79.9 \\
69.7\end{array}$ & $\begin{array}{l}320.7 \\
279.7 \\
407.7 \\
394.4 \\
392.7\end{array}$ & $\begin{array}{l}25.80 \\
23.16 \\
19.33 \\
18.98 \\
22.01\end{array}$ & $\begin{array}{l}33.30 \\
30.99 \\
28.95 \\
30.54 \\
32.15\end{array}$ & $\begin{array}{r}14.04 \\
15.91 \\
9.99 \\
7.55 \\
9.35\end{array}$ & $\begin{array}{l}37.3 \\
47.0 \\
41.1 \\
43.5 \\
33.8\end{array}$ & $\begin{array}{l}61.9 \\
84.3 \\
61.0 \\
70.7 \\
69.6\end{array}$ & $\begin{array}{l}24.4 \\
13.0 \\
18.6 \\
19.3 \\
17.1\end{array}$ & $\begin{array}{r}1.35 \\
2.46 \\
1.56 \\
.84 \\
1.21\end{array}$ & $\begin{array}{l}2.88 \\
5.62 \\
2.89 \\
1.73 \\
3.38\end{array}$ & $\begin{array}{l}.45 \\
.76 \\
.51 \\
.27 \\
.40\end{array}$ \\
\hline $\begin{array}{l}276 \\
277 \\
278 \\
279 \\
280\end{array}$ & $\begin{array}{r}1.5 \\
43.8 \\
66.6 \\
74.0 \\
63.9\end{array}$ & $\begin{array}{l}159.2 \\
297.4 \\
409.6 \\
391.9 \\
393.8\end{array}$ & $\begin{array}{l}19.57 \\
19.20 \\
15.26 \\
15.12 \\
18.65\end{array}$ & $\begin{array}{l}24.17 \\
22.30 \\
22.19 \\
25.79 \\
28.31\end{array}$ & $\begin{array}{r}11.77 \\
16.36 \\
6.31 \\
3.10 \\
7.04\end{array}$ & $\begin{array}{l}36.0 \\
43.3 \\
53.4 \\
45.2 \\
31.5\end{array}$ & $\begin{array}{l}60.5 \\
54.6 \\
79.8 \\
79.4 \\
63.2\end{array}$ & $\begin{array}{l}15.3 \\
31.4 \\
31.0 \\
20.5 \\
13.6\end{array}$ & $\begin{array}{r}1.56 \\
3.68 \\
2.21 \\
.93 \\
1.49\end{array}$ & $\begin{array}{l}3.52 \\
4.89 \\
4.69 \\
1.60 \\
2.61\end{array}$ & $\begin{array}{r}.35 \\
2.10 \\
.33 \\
.39 \\
.56\end{array}$ \\
\hline $\begin{array}{l}281 \\
282 \\
283 \\
284 \\
285\end{array}$ & $\begin{array}{l}57.5 \\
66.4 \\
65.0 \\
56.3 \\
55.4\end{array}$ & $\begin{array}{l}379.0 \\
374.2 \\
372.1 \\
365.2 \\
385.1\end{array}$ & $\begin{array}{l}19.83 \\
16.92 \\
17.75 \\
19.42 \\
20.05\end{array}$ & $\begin{array}{l}29.36 \\
30.53 \\
29.69 \\
29.66 \\
27.93\end{array}$ & $\begin{array}{l}8.61 \\
4.20 \\
6.04 \\
7.27 \\
6.89\end{array}$ & $\begin{array}{l}29.4 \\
33.7 \\
30.1 \\
29.9 \\
27.1\end{array}$ & $\begin{array}{l}59.6 \\
62.2 \\
50.1 \\
61.0 \\
55.2\end{array}$ & $\begin{array}{l}13.9 \\
12.0 \\
14.3 \\
13.6 \\
13.7\end{array}$ & $\begin{array}{r}1.64 \\
.81 \\
1.32 \\
1.10 \\
3.17\end{array}$ & $\begin{array}{l}3.51 \\
1.94 \\
3.82 \\
2.07 \\
5.28\end{array}$ & $\begin{array}{l}.46 \\
.41 \\
.39 \\
.35 \\
.46\end{array}$ \\
\hline
\end{tabular}




\begin{tabular}{|c|c|c|c|c|c|c|c|c|c|c|c|}
\hline \multirow{2}{*}{$\begin{array}{l}\text { Calendar } \\
\text { day }\end{array}$} & \multicolumn{2}{|c|}{$\begin{array}{l}\text { Net radiation, in watts } \\
\text { per square meter }\end{array}$} & \multicolumn{3}{|c|}{$\begin{array}{c}\text { Air temperature at } 1.25 \text { meters, } \\
\text { in degrees Celsius }\end{array}$} & \multicolumn{3}{|c|}{$\begin{array}{l}\text { Humidity at } 1.25 \text { meters, } \\
\text { in degrees Celsius }\end{array}$} & \multicolumn{3}{|c|}{$\begin{array}{l}\text { Windspeed at } 1.25 \text { meters, } \\
\text { in meters per second }\end{array}$} \\
\hline & Mean & Maximum & Mean & Maximum & Minimum & Mean & Maximum & Minimum & Mean & Maximum & Minimum \\
\hline $\begin{array}{l}286 \\
287 \\
288 \\
289 \\
290\end{array}$ & $\begin{array}{l}52.1 \\
35.5 \\
48.9 \\
50.8 \\
56.0\end{array}$ & $\begin{array}{l}370.8 \\
313.2 \\
343.4 \\
358.3 \\
354.6\end{array}$ & $\begin{array}{l}16.16 \\
17.55 \\
13.49 \\
11.87 \\
11.03\end{array}$ & $\begin{array}{l}24.11 \\
22.51 \\
18.73 \\
20.28 \\
21.23\end{array}$ & $\begin{array}{r}5.76 \\
12.49 \\
5.83 \\
2.97 \\
1.94\end{array}$ & $\begin{array}{l}37.0 \\
41.2 \\
37.7 \\
36.4 \\
42.8\end{array}$ & $\begin{array}{l}69.8 \\
53.7 \\
60.5 \\
55.2 \\
64.1\end{array}$ & $\begin{array}{l}17.3 \\
32.4 \\
20.8 \\
20.2 \\
22.2\end{array}$ & $\begin{array}{r}1.56 \\
1.84 \\
2.72 \\
1.71 \\
.93\end{array}$ & $\begin{array}{l}2.69 \\
3.11 \\
4.79 \\
2.89 \\
2.19\end{array}$ & $\begin{array}{r}0.52 \\
.71 \\
.77 \\
.44 \\
.23\end{array}$ \\
\hline $\begin{array}{l}291 \\
292 \\
293 \\
294 \\
295\end{array}$ & $\begin{array}{l}56.7 \\
53.7 \\
51.2 \\
51.5 \\
36.0\end{array}$ & $\begin{array}{l}352.8 \\
343.7 \\
334.9 \\
334.0 \\
261.0\end{array}$ & $\begin{array}{l}12.02 \\
12.48 \\
12.80 \\
12.73 \\
12.99\end{array}$ & $\begin{array}{l}23.51 \\
25.03 \\
25.67 \\
27.17 \\
26.60\end{array}$ & $\begin{array}{r}1.68 \\
2.66 \\
2.17 \\
.27 \\
2.10\end{array}$ & $\begin{array}{l}38.0 \\
35.4 \\
33.6 \\
34.7 \\
33.8\end{array}$ & $\begin{array}{l}63.5 \\
58.1 \\
50.3 \\
65.6 \\
62.3\end{array}$ & $\begin{array}{l}19.0 \\
15.9 \\
16.3 \\
13.9 \\
13.6\end{array}$ & $\begin{array}{l}.94 \\
.87 \\
.78 \\
.75 \\
.66\end{array}$ & $\begin{array}{l}1.84 \\
1.70 \\
1.36 \\
1.41 \\
1.35\end{array}$ & $\begin{array}{l}.45 \\
.35 \\
.39 \\
.33 \\
.31\end{array}$ \\
\hline $\begin{array}{l}296 \\
297 \\
298 \\
299 \\
300\end{array}$ & $\begin{array}{l}48.0 \\
51.9 \\
50.7 \\
46.8 \\
42.8\end{array}$ & $\begin{array}{l}281.2 \\
322.7 \\
324.3 \\
319.0 \\
348.1\end{array}$ & $\begin{array}{l}14.72 \\
16.28 \\
15.28 \\
14.99 \\
15.12\end{array}$ & $\begin{array}{l}26.26 \\
27.30 \\
28.20 \\
28.15 \\
27.56\end{array}$ & $\begin{array}{l}1.25 \\
6.30 \\
4.96 \\
4.66 \\
4.78\end{array}$ & $\begin{array}{l}30.5 \\
33.5 \\
39.2 \\
37.4 \\
35.9\end{array}$ & $\begin{array}{l}56.1 \\
52.5 \\
59.9 \\
55.8 \\
52.4\end{array}$ & $\begin{array}{l}14.7 \\
16.2 \\
17.2 \\
17.2 \\
18.3\end{array}$ & $\begin{array}{l}.87 \\
.87 \\
.77 \\
.79 \\
.74\end{array}$ & $\begin{array}{l}1.73 \\
1.56 \\
1.43 \\
1.31 \\
1.78\end{array}$ & $\begin{array}{l}.30 \\
.35 \\
.28 \\
.32 \\
.36\end{array}$ \\
\hline $\begin{array}{l}301 \\
302 \\
303 \\
304 \\
305\end{array}$ & $\begin{array}{l}45.3 \\
40.9 \\
30.7 \\
37.2 \\
23.1\end{array}$ & $\begin{array}{l}319.1 \\
316.7 \\
317.7 \\
306.0 \\
288.0\end{array}$ & $\begin{array}{l}13.85 \\
14.61 \\
14.04 \\
11.33 \\
15.63\end{array}$ & $\begin{array}{l}27.16 \\
26.38 \\
24.15 \\
25.76 \\
26.14\end{array}$ & $\begin{array}{l}3.90 \\
2.78 \\
4.23 \\
-.07 \\
1.43\end{array}$ & $\begin{array}{l}39.3 \\
32.9 \\
23.9 \\
24.2 \\
19.0\end{array}$ & $\begin{array}{l}-69.2 \\
57.0 \\
44.9 \\
62.3 \\
32.3\end{array}$ & $\begin{array}{r}16.0 \\
18.0 \\
11.9 \\
4.7 \\
10.4\end{array}$ & $\begin{array}{r}.78 \\
1.37 \\
1.53 \\
.75 \\
2.43\end{array}$ & $\begin{array}{l}1.45 \\
2.71 \\
2.67 \\
1.65 \\
5.01\end{array}$ & $\begin{array}{l}.31 \\
.48 \\
.47 \\
.37 \\
.54\end{array}$ \\
\hline $\begin{array}{l}306 \\
307 \\
308 \\
309 \\
310\end{array}$ & $\begin{array}{r}8.5 \\
20.2 \\
28.0 \\
40.3 \\
31.1\end{array}$ & $\begin{array}{l}167.1 \\
296.5 \\
307.3 \\
297.6 \\
291.8\end{array}$ & $\begin{array}{r}14.43 \\
7.60 \\
5.04 \\
8.68 \\
12.7\end{array}$ & $\begin{array}{l}18.70 \\
12.52 \\
13.62 \\
18.53 \\
23.26\end{array}$ & $\begin{array}{r}8.31 \\
2.47 \\
-1.91 \\
-.22 \\
1.51\end{array}$ & $\begin{array}{l}40.4 \\
37.8 \\
31.7 \\
26.4 \\
28.7\end{array}$ & $\begin{array}{l}56.5 \\
69.2 \\
45.9 \\
59.8 \\
53.6\end{array}$ & $\begin{array}{l}26.4 \\
18.2 \\
15.1 \\
11.8 \\
13.3\end{array}$ & $\begin{array}{l}3.12 \\
2.77 \\
1.20 \\
1.15 \\
1.37\end{array}$ & $\begin{array}{l}5.15 \\
4.69 \\
2.55 \\
3.35 \\
3.37\end{array}$ & $\begin{array}{r}.38 \\
1.49 \\
.37 \\
.33 \\
.35\end{array}$ \\
\hline $\begin{array}{l}311 \\
312 \\
313 \\
314 \\
315\end{array}$ & $\begin{array}{r}9.2 \\
41.4 \\
30.1 \\
4.4 \\
30.7\end{array}$ & $\begin{array}{l}200.8 \\
286.5 \\
305.3 \\
194.7 \\
261.9\end{array}$ & $\begin{array}{r}10.79 \\
10.73 \\
12.95 \\
10.36 \\
5.78\end{array}$ & $\begin{array}{l}19.65 \\
19.46 \\
20.58 \\
15.19 \\
13.57\end{array}$ & $\begin{array}{r}3.46 \\
3.27 \\
2.85 \\
3.50 \\
-3.23\end{array}$ & $\begin{array}{l}29.5 \\
45.4 \\
44.4 \\
61.8 \\
70.2\end{array}$ & $\begin{array}{l}48.0 \\
66.4 \\
68.0 \\
83.1 \\
92.9\end{array}$ & $\begin{array}{l}14.1 \\
27.5 \\
28.4 \\
39.9 \\
37.3\end{array}$ & $\begin{array}{r}1.18 \\
.99 \\
2.48 \\
2.89 \\
.89\end{array}$ & $\begin{array}{l}2.69 \\
1.99 \\
5.66 \\
5.09 \\
1.79\end{array}$ & $\begin{array}{l}.39 \\
.44 \\
.40 \\
.46 \\
.34\end{array}$ \\
\hline $\begin{array}{l}316 \\
317 \\
318 \\
319 \\
320\end{array}$ & $\begin{array}{l}46.2 \\
12.2 \\
15.3 \\
28.4 \\
27.6\end{array}$ & $\begin{array}{l}294.2 \\
284.0 \\
280.9 \\
284.3 \\
276.4\end{array}$ & $\begin{array}{l}5.40^{\circ} \\
7.73 \\
5.52 \\
5.45 \\
9.67\end{array}$ & $\begin{array}{l}14.74 \\
13.47 \\
13.64 \\
15.64 \\
14.54\end{array}$ & $\begin{array}{r}-3.25 \\
3.44 \\
-3.50 \\
-8.20 \\
.02\end{array}$ & $\begin{array}{l}66.2 \\
33.0 \\
34.3 \\
37.5 \\
33.7\end{array}$ & $\begin{array}{l}95.1 \\
61.0 \\
62.0 \\
75.7 \\
60.0\end{array}$ & $\begin{array}{l}33.1 \\
15.3 \\
17.2 \\
15.0 \\
21.2\end{array}$ & $\begin{array}{l}1.29 \\
2.52 \\
1.91 \\
1.24 \\
2.32\end{array}$ & $\begin{array}{l}3.02 \\
4.01 \\
3.03 \\
2.90 \\
4.08\end{array}$ & $\begin{array}{r}.26 \\
1.24 \\
.43 \\
.29 \\
.65\end{array}$ \\
\hline $\begin{array}{l}321 \\
322 \\
323 \\
324 \\
325\end{array}$ & $\begin{array}{l}23.6 \\
11.2 \\
14.4 \\
22.5 \\
18.9\end{array}$ & $\begin{array}{l}286.7 \\
208.1 \\
286.4 \\
290.1 \\
249.3\end{array}$ & $\begin{array}{l}5.46 \\
2.07 \\
3.54 \\
1.68 \\
3.22\end{array}$ & $\begin{array}{r}13.73 \\
6.54 \\
11.50 \\
12.27 \\
13.99\end{array}$ & $\begin{array}{r}-5.34 \\
-.75 \\
-1.08 \\
-6.46 \\
-6.45\end{array}$ & $\begin{array}{l}49.8 \\
62.8 \\
34.2 \\
57.6 \\
56.5\end{array}$ & $\begin{array}{l}75.5 \\
97.0 \\
53.9 \\
84.8 \\
89.0\end{array}$ & $\begin{array}{l}33.0 \\
32.7 \\
18.0 \\
20.1 \\
21.6\end{array}$ & $\begin{array}{r}2.63 \\
2.25 \\
3.01 \\
.74 \\
.95\end{array}$ & $\begin{array}{l}4.90 \\
4.73 \\
5.03 \\
1.18 \\
2.85\end{array}$ & $\begin{array}{l}.48 \\
.74 \\
.69 \\
.24 \\
.24\end{array}$ \\
\hline
\end{tabular}


Table 6. Daily mean, maximum, and minimum micrometeorological data measured by micromet station at Rogers Spring Site 2, 1994-Continued

\begin{tabular}{|c|c|c|c|c|c|c|c|c|c|c|c|}
\hline \multirow{2}{*}{$\begin{array}{c}\text { Calendar } \\
\text { day }\end{array}$} & \multicolumn{2}{|c|}{$\begin{array}{l}\text { Net radiation, in watts } \\
\text { per square meter }\end{array}$} & \multicolumn{3}{|c|}{$\begin{array}{l}\text { Air temperature at } 1.25 \text { meters, } \\
\text { in degrees Celsius }\end{array}$} & \multicolumn{3}{|c|}{$\begin{array}{l}\text { Humidity at } 1.25 \text { meters, } \\
\text { in degrees Celsius }\end{array}$} & \multicolumn{3}{|c|}{$\begin{array}{l}\text { Windspeed at } 1.25 \text { meters, } \\
\text { in meters per second }\end{array}$} \\
\hline & Mean & Maximum & Mean & Maximum & Minimum & Mean & Maximum & Minimum & Mean & Maximum & Minimum \\
\hline $\begin{array}{l}326 \\
327 \\
328 \\
329 \\
330\end{array}$ & $\begin{array}{r}12.2 \\
18.5 \\
22.0 \\
14.3 \\
-12.1\end{array}$ & $\begin{array}{l}278.3 \\
259.9 \\
217.4 \\
267.6 \\
262.3\end{array}$ & $\begin{array}{l}7.49 \\
3.25 \\
2.72 \\
8.07 \\
6.58\end{array}$ & $\begin{array}{l}14.72 \\
15.72 \\
14.88 \\
19.03 \\
10.83\end{array}$ & $\begin{array}{r}1.76 \\
-6.20 \\
-7.66 \\
-4.86 \\
.29\end{array}$ & $\begin{array}{l}41.4 \\
48.2 \\
51.7 \\
48.1 \\
43.8\end{array}$ & $\begin{array}{l}60.0 \\
82.3 \\
84.0 \\
76.6 \\
68.0\end{array}$ & $\begin{array}{l}23.8 \\
17.5 \\
20.1 \\
20.7 \\
20.8\end{array}$ & $\begin{array}{r}2.68 \\
.82 \\
.63 \\
3.84 \\
4.00\end{array}$ & $\begin{array}{l}4.18 \\
2.02 \\
1.07 \\
6.85 \\
5.81\end{array}$ & $\begin{array}{r}0.83 \\
.39 \\
.31 \\
.50 \\
2.05\end{array}$ \\
\hline $\begin{array}{l}331 \\
332 \\
333 \\
334 \\
335\end{array}$ & $\begin{array}{l}12.6 \\
14.4 \\
19.3 \\
22.8 \\
20.5\end{array}$ & $\begin{array}{l}250.1 \\
248.7 \\
245.4 \\
243.5 \\
241.2\end{array}$ & $\begin{array}{r}.80 \\
1.99 \\
3.51 \\
4.51 \\
4.54\end{array}$ & $\begin{array}{r}9.08 \\
13.23 \\
14.88 \\
18.83 \\
18.03\end{array}$ & $\begin{array}{l}-5.80 \\
-8.50 \\
-5.16 \\
-5.35 \\
-5.57\end{array}$ & $\begin{array}{l}39.8 \\
39.3 \\
39.4 \\
43.7 \\
45.8\end{array}$ & $\begin{array}{l}57.8 \\
59.1 \\
70.4 \\
72.3 \\
72.7\end{array}$ & $\begin{array}{l}16.7 \\
19.7 \\
13.9 \\
17.4 \\
19.1\end{array}$ & $\begin{array}{r}1.18 \\
1.18 \\
.86 \\
.72 \\
.77\end{array}$ & $\begin{array}{l}2.03 \\
2.48 \\
1.85 \\
1.24 \\
1.37\end{array}$ & $\begin{array}{l}.46 \\
.60 \\
.31 \\
.30 \\
.35\end{array}$ \\
\hline $\begin{array}{l}336 \\
337 \\
338 \\
339 \\
340\end{array}$ & $\begin{array}{r}19.6 \\
.5 \\
-4.1 \\
21.0 \\
11.7\end{array}$ & $\begin{array}{r}236.9 \\
103.6 \\
87.1 \\
231.6 \\
241.1\end{array}$ & $\begin{array}{r}5.30 \\
8.78 \\
10.55 \\
8.69 \\
7.24\end{array}$ & $\begin{array}{l}17.40 \\
15.16 \\
16.56 \\
17.39 \\
15.64\end{array}$ & $\begin{array}{r}-5.01 \\
-0.96 \\
5.32 \\
1.93 \\
-1.29\end{array}$ & $\begin{array}{l}45.2 \\
41.3 \\
36.0 \\
47.6 \\
59.1\end{array}$ & $\begin{array}{r}67.8 \\
71.2 \\
49.9 \\
72.1 \\
\times 82.4\end{array}$ & $\begin{array}{l}18.4 \\
26.7 \\
25.9 \\
27.3 \\
33.7\end{array}$ & $\begin{array}{r}.82 \\
1.20 \\
1.45 \\
.96 \\
1.38\end{array}$ & $\begin{array}{l}1.52 \\
2.90 \\
3.60 \\
1.59 \\
3.48\end{array}$ & $\begin{array}{l}.41 \\
.45 \\
.45 \\
.49 \\
.39\end{array}$ \\
\hline $\begin{array}{l}341 \\
342 \\
343 \\
344 \\
345\end{array}$ & $\begin{array}{r}8.2 \\
.9 \\
8.0 \\
-1.6 \\
7.4\end{array}$ & $\begin{array}{l}240.6 \\
234.6 \\
228.4 \\
165.3 \\
167.8\end{array}$ & $\begin{array}{r}5.96 \\
3.83 \\
-.10 \\
-1.40 \\
-1.37\end{array}$ & $\begin{array}{r}14.68 \\
10.55 \\
12.89 \\
9.46 \\
9.16\end{array}$ & $\begin{array}{r}-1.83 \\
-5.99 \\
-9.71 \\
-9.50 \\
-10.52\end{array}$ & $\begin{array}{l}47.5 \\
28.2 \\
35.0 \\
41.6 \\
40.8\end{array}$ & $\begin{array}{l}90.1 \\
56.1 \\
59.6 \\
72.2 \\
69.1\end{array}$ & $\begin{array}{r}18.8 \\
11.8 \\
9.7 \\
19.1 \\
18.0\end{array}$ & $\begin{array}{r}1.76 \\
2.36 \\
1.17 \\
.80 \\
.75\end{array}$ & $\begin{array}{l}3.39 \\
4.12 \\
2.61 \\
1.45 \\
1.29\end{array}$ & $\begin{array}{l}.44 \\
.34 \\
.45 \\
.44 \\
.42\end{array}$ \\
\hline $\begin{array}{l}346 \\
347 \\
348 \\
349 \\
350\end{array}$ & $\begin{array}{r}20.2 \\
-4.2 \\
25.1 \\
16.1 \\
18.9\end{array}$ & $\begin{array}{l}231.4 \\
164.2 \\
214.3 \\
228.4 \\
231.7\end{array}$ & $\begin{array}{l}6.76 \\
6.11 \\
1.72 \\
1.16 \\
2.07\end{array}$ & $\begin{array}{l}15.72 \\
11.85 \\
10.14 \\
12.64 \\
15.36\end{array}$ & $\begin{array}{l}-4.21 \\
-3.50 \\
-7.34 \\
-6.89 \\
-7.61\end{array}$ & $\begin{array}{l}32.0 \\
65.1 \\
61.6 \\
57.7 \\
52.9\end{array}$ & $\begin{array}{l}61.3 \\
91.9 \\
91.5 \\
83.6 \\
76.8\end{array}$ & $\begin{array}{l}16.4 \\
38.5 \\
36.4 \\
29.2 \\
24.2\end{array}$ & $\begin{array}{r}2.58 \\
2.01 \\
.86 \\
.71 \\
.74\end{array}$ & $\begin{array}{l}4.82 \\
4.71 \\
1.47 \\
1.28 \\
1.43\end{array}$ & $\begin{array}{l}.44 \\
.40 \\
.48 \\
.33 \\
.29\end{array}$ \\
\hline $\begin{array}{l}351 \\
352 \\
353 \\
354 \\
355\end{array}$ & $\begin{array}{l}19.2 \\
17.8 \\
14.9 \\
13.7 \\
21.3\end{array}$ & $\begin{array}{l}233.9 \\
229.7 \\
226.8 \\
225.4 \\
225.1\end{array}$ & $\begin{array}{l}3.95 \\
3.34 \\
5.17 \\
4.60 \\
5.14\end{array}$ & $\begin{array}{l}18.10 \\
17.04 \\
18.66 \\
19.71 \\
20.26\end{array}$ & $\begin{array}{l}-5.77 \\
-6.02 \\
-4.74 \\
-6.38 \\
-6.36\end{array}$ & $\begin{array}{l}49.4 \\
50.5 \\
43.8 \\
41.4 \\
39.1\end{array}$ & $\begin{array}{l}74.6 \\
73.5 \\
76.5 \\
71.0 \\
71.0\end{array}$ & $\begin{array}{l}23.4 \\
22.7 \\
14.7 \\
14.8 \\
12.6\end{array}$ & $\begin{array}{l}.79 \\
.81 \\
.83 \\
.76 \\
.69\end{array}$ & $\begin{array}{l}1.22 \\
1.28 \\
1.38 \\
1.18 \\
1.10\end{array}$ & $\begin{array}{l}.34 \\
.37 \\
.38 \\
.47 \\
.35\end{array}$ \\
\hline $\begin{array}{l}356 \\
357 \\
358 \\
359 \\
360\end{array}$ & $\begin{array}{r}6.4 \\
19.8 \\
9.0 \\
37.2 \\
16.8\end{array}$ & $\begin{array}{r}101.4 \\
163.0 \\
78.8 \\
261.3 \\
272.4\end{array}$ & $\begin{array}{r}9.84 \\
12.37 \\
10.37 \\
10.10 \\
10.20\end{array}$ & $\begin{array}{l}17.84 \\
15.73 \\
12.10 \\
14.43 \\
17.68\end{array}$ & $\begin{array}{r}.26 \\
10.30 \\
8.83 \\
7.29 \\
5.85\end{array}$ & $\begin{array}{l}32.5 \\
54.0 \\
87.6 \\
79.9 \\
60.6\end{array}$ & $\begin{array}{l}54.2 \\
68.8 \\
99.0 \\
99.9 \\
78.9\end{array}$ & $\begin{array}{l}15.6 \\
34.7 \\
70.1 \\
61.2 \\
36.1\end{array}$ & $\begin{array}{l}1.25 \\
1.36 \\
1.82 \\
2.05 \\
2.43\end{array}$ & $\begin{array}{l}2.86 \\
3.26 \\
3.90 \\
3.62 \\
3.87\end{array}$ & $\begin{array}{l}.35 \\
.32 \\
.56 \\
.76 \\
.66\end{array}$ \\
\hline $\begin{array}{l}361 \\
362 \\
363 \\
364 \\
365\end{array}$ & $\begin{array}{l}23.2 \\
34.3 \\
13.2 \\
15.9 \\
36.5\end{array}$ & $\begin{array}{l}257.6 \\
257.0 \\
151.0 \\
265.7 \\
259.1\end{array}$ & $\begin{array}{l}7.57 \\
9.21 \\
6.59 \\
5.56 \\
2.69\end{array}$ & $\begin{array}{l}16.35 \\
16.48 \\
10.10 \\
13.08 \\
11.52\end{array}$ & $\begin{array}{r}.11 \\
3.58 \\
4.55 \\
-2.28 \\
-4.23\end{array}$ & $\begin{array}{l}72.6 \\
70.4 \\
83.2 \\
62.9 \\
76.7\end{array}$ & $\begin{array}{l}94.5 \\
94.1 \\
95.8 \\
94.1 \\
94.2\end{array}$ & $\begin{array}{l}40.2 \\
40.2 \\
69.0 \\
28.7 \\
44.0\end{array}$ & $\begin{array}{r}.82 \\
2.35 \\
1.22 \\
1.87 \\
.57\end{array}$ & $\begin{array}{r}1.67 \\
4.97 \\
2.62 \\
3.33 \\
.85\end{array}$ & $\begin{array}{l}.30 \\
.45 \\
.37 \\
.30 \\
.22\end{array}$ \\
\hline
\end{tabular}


NATIONAL RESOURCES LIBRARY US DEPT OF THE INTERIOR

WASHINGTON DC 20240

JACK RACHLIN

MILITARY GEOLOGY UNIT

US GEOLOGICAL SURVEY

928 NATIONAL CENTER

RESTON VA 22092

OSTI

US DEPT OF ENERGY

PO BOX 62

OAK RIDGE

TN 37831

MARTHA GARCIA

USGS GEOLOGIC DIVISION

BOX 25046 MS 913

DENVER FEDERAL CENTER

DENVER

CO 80225

JOHN R BANNISTER DIV 7111

SANDIA NATL LABS

PO BOX 5800

ALBUQUERQUE NM 87185

JERRY MERCER

SANDIA NATL LABS

PO BOX 5800

ALBUQUERQUE

NM 87185

BRUCE M CROWE

LOS ALAMOS NATIONAL LAB

PO BOX 1663

LOS ALAMOS

NM 87545

W M BRUNISH

LOS ALAMOS NATL LAB

PO BOX 1663

MS 5000

LOS ALAMOS

NM 87545

RICK WARREN

LOS ALAMOS NATL LAB

PO BOX 1663

LOS ALAMOS NM 87545

MS BL HARRIS-WEST NVCG DEFENSE NUCLEAR AGENCY

PO BOX 208 MS 645

MERCURY

NV 89023
TE KENNEDY SPTD

DEFENSE NUCLEAR AGENCY

WASHINGTON

DC 20305-1000

SERIAL RECORDS UNIT LIBRARY

US GEOLOGICAL SURVEY

950 NATIONAL CENTER

RESTON

VA 22092

LIBRARY

WATERWAYS EXPERIMENT STATION

US ARMY CORPS OF ENGINEERS

PO BOX 631

VICKSBURG

MS $39180-0631$

LIBRARY

US GEOLOGICAL SURVEY

2255 N GEMINI DR

FLAGSTAFF

AZ 86001

TO HUNTER

SANDIA NATIONAL LABORATORY PO BOX 5800 ORG 6300

ALBUQUERQUE NM 87185

REPORT COLLECTION

RESEARCH LIBRARY

PO BOX 7113

MS P362

LOS ALAMOS

NM $87544-7113$

NANCY MARUSAK ESS 5

LOS ALAMOS NATL LAB

PO BOX 1663 MS F665

LOS ALAMOS

NM 87545

JN PETERSON J 6

LOS ALAMOS NATL LAB

PO BOX 1663

MS C925

LOS ALAMOS

NM 87545

WARD HAWKINS

LOS ALAMOS NATIONAL LAB

PO BOX 1663

LOS ALAMOS

NM 87545

SLDRELLACK JR

RAYTHEON SERVICES NEVADA PO BOX 328

MERCURY

NV 89023
DIRECTOR

ATTN DDST T

DEFENSE NUCLEAR AGENCY

WASHINGTON

DC 20305-100

$M$ W REYNOLDS CHIEF

OFFICE OF REGIONAL GEOLOGY

US GEOLOGICAL SURVEY

908 NATIONAL CENTER

RESTON

VA 22092

JIM COLE

USGS GEOLOGIC DIVISION

BOX 25046 MS 913

DENVER FEDERAL CENTER

DENVER

CO 80225

ATTN CONTAINMENT SCIEN FCTK

FIELD COMMAND

DEFENSE NUCLEAR AGENCY

KIRTLAND AFB

NM $87115-5001$

C W SMITH DIV 7112

SANDIA NATL LABS

PO BOX 5800

ALBUQUERQUE NM 87185

A H COGBILL

LOS ALAMOS NATL LAB

PO BOX 1663

MS F659

LOS ALAMOS NM 87545

T A WEAVER G 5

LOS ALAMOS NATL LAB

PO BOX 1663 MS 586

LOS ALAMOS

NM 87545

JOE THOMPSON

LOS ALAMOS NATIONAL LAB

PO BOX 1663

LOS ALAMOS

NM 87545

GEOLOGIC DATA CENTER

US GEOLOGICAL SURVEY

PO BOX 327

MERCURY

NV 89023

DIRECTOR

NTS SUPPORT OFFICE

US DEPT OF ENERGY

PO BOX 435 MS 701

MERCURY

NV 89023 
GRANT T BRUESCH MANAGER

TECH SUPPORT OFFICE

RAYTHEON SERVICES NEVADA

PO BOX 328

MERCURY

NV 89023

JOHN EBERLIN

IT CORP

4330 S VALLEY VIEW BLVD

STE 112

LAS VEGAS

NV 89103

\section{A TRUDEAU}

US GEOLOGICAL SURVEY

6770 S PARADISE RD

LAS VEGAS

NV 89119

LIBRARY

US GEOLOGICAL SURVEY

6770 S PARADISE RD

LAS VEGAS

NV 89119

ROGER L JACOBSEN

DESERT RESEARCH INSTITUTE

755 E FLAMINGO RD

LAS VEGAS NV 89119

JM SOESBE

NEVADA OPERATIONS OFFICE

US DEPT OF ENERGY

PO BOX 98518

LAS VEGAS

NV $89193-8518$

KENNETH W POWERS

DIRECTOR CONTRACTS DIV

US DEPT OF ENERGY

PO BOX 98518

LAS VEGAS

NV $89193-8518$

CHARLES MCWILLIAM

NEVADA OPERATIONS OFFICE

US DEPT OF ENERGY

PO BOX 98518

LAS VEGAS

NV $89193-8518$

D R ELLE

NEVADA OPERATIONS OFFICE

US DEPT OF ENERGY

PO BOX 98518

LAS VEGAS

NV $89193-8518$

JC BURROWS

TECH PROJ BRANCH-DOE

NEVADA OPERATIONS OFFICE

PO BOX 98518

LAS VEGAS

NV $89193-8518$
CETO LIBRARY

PO BOX 495

MERCURY

NV $89023-0495$

RKWADDELL

GEOTRANS

4330 S VALLEY VIEW BLVD

STE 112

LAS VEGAS NV 89103

GARY RUSSELL

US GEOLOGICAL SURVEY

6770 S PARADISE RD

LAS VEGAS

NV 89119

PAUL SEABER

DESERT RESEARCH INSTITUTE

755 E FLAMINGO RD

LAS VEGAS . NV 89119

GLDIXON

US GEOLOGICAL SURVEY

GEOLOGIC DIVISION

6770 S PARADISE RD

LAS VEGAS NV 89119

\section{J S LEDBETTER}

CONTRACTS DIV

US DEPT OF ENERGY

PO BOX 98518

LAS VEGAS

NV $89193-8518$

$M G$ PINON

RESOURCE MANAGEMENT DIV

US DEPT OF ENERGY

PO BOX 98518

LAS VEGAS

NV $89193-8518$

$S$ H LEEDOM TEST TECH

NEVADA OPERATIONS OFFICE

US DEPT OF ENERGY

PO BOX 98518

LAS VEGAS

NV $89193-8518$

D W DUNCAN

ENV RES AND WASTE MGT DIV

US DEPT OF ENERGY

PO BOX 98518

LAS VEGAS

NV $89193-8518$

JAMES O'DONNELL

NEVADA OPERATIONS OFFICE

US DEPT OF ENERGY

PO BOX 98518

LAS VEGAS
MIKE O'HAGAN

4330 S VALLEY VIEW BLVD

STE 112

LAS VEGAS

NV 89103

CHUCK RUSSELL

DESERT RESEARCH INSTITUTE 755 E FLAMINGO RD

LAS VEGAS

NV 89119

JOE YEASTED

IT CORP C/O DRI

755 E FLAMINGO RD

LAS VEGAS

NV 89119

RANDY LACZNIAK

US GEOLOGICAL SURVEY

6770 S PARADISE RD

LAS VEGAS

NV 89119

DAVID L MARKS DIRECTOR

RESOURCE MANAGEMENT DIV

US DEPT OF ENERGY

PO BOX 98518

LAS VEGAS

NV $89193-8518$

JANET FROST

RESOURCE MANAGEMENT DIV

US DEPT OF ENERGY

PO BOX 98518

LAS VEGAS

NV $89193-8518$

R J BLAKLEY

NEVADA OPERATIONS OFFICE

US DEPT OF ENERGY

PO BOX 98518

LAS VEGAS

NV $89193-8518$

STEPHEN A MELLINGTON

ENV RES AND WASTE MGT DIV

US DEPT OF ENERGY

PO BOX 98518

LAS VEGAS

NV $89193-8518$

E WAYNE ADAMS

SAFEGUARDS AND SECURITY DIV US DEPT OF ENERGY

PO BOX 98518

LAS VEGAS

NV $89193-8518$

KK GOETZ

RESOURCE MANAGEMENT DIV

US DEPT OF ENERGY

PO BOX 98518

LAS VEGAS

NV $89193-8518$ 
LEAH DEVER

NEVADA OPERATIONS OFFICE

US DEPT OF ENERGY

PO BOX 98518

LAS VEGAS

NV $89193-8518$

PAUL KUNICHIKA

NEVADA OPERATIONS OFFICE

US DEPT OF ENERGY

PO BOX 98518

LAS VEGAS

NV $89193-8518$

STEVE LAWRENCE

ENV RES AND WASTE MGT DIV

US DEPT OF ENERGY

PO BOX 98518

LAS VEGAS

NV $89193-8518$

RON HESS

GEOL INF SPECIALIST

NEVADA BUR OF MINES AND GEOL

UNIV OF NEVADA

RENO

NV $89557-0088$

DAVE SMITH

LAWRENCE LIVERMORE NTL LAB

PO BOX 808

LIVERMORE

CA 94551

J L WAGONER L 221

LAWRENCE LIVERMORE NTL LAB

PO BOX 808

LIVERMORE

CA 94551
NEVADA TECHNICAL LIBRARY

NEVADA OPERATIONS OFFICE

US DEPT OF ENERGY

PO BOX 98518

LAS VEGAS

NV $89193-8518$

RICHARD NAVARRO

NEVADA OPERATIONS OFFICE

US DEPT OF ENERGY

PO BOX 98518

LAS VEGAS

NV $89193-8518$

TECHNICAL INFO OFFICER PIB

NEVADA OPERATIONS OFFICE

US DEPT OF ENERGY

PO BOX 98518

LAS VEGAS

NV $89193-8518$

H L MCKAGUE L 221

LAWRENCE LIVERMORE NTL LAB PO BOX 808

LIVERMORE

CA 94551

DR NORMAN R BURKHARD L 221 LAWRENCE LIVERMORE NTL LAB

PO BOX 808

LIVERMORE

CA 94551

MS GA PAWLOSKI L 221

LAWRENCE LIVERMORE NTL LAB

PO BOX 808

LIVERMORE
NEWS TEST TECH BRANCH

NEVADA OPERATIONS OFFICE

US DEPT OF ENERGY

PO BOX 98518

LAS VEGAS

NV $89193-851$

RICHARD PEARL

NEVADA OPERATIONS OFFICE

US DEPT OF ENERGY

PO BOX 89158

LAS VEGAS

NV $89193-851$

DESERT RESEARCH INSTITUTE

UNIV OF NEVADA

PO BOX 8086 UNIV STA

RENO

NV 89507

SEISMIC LIBRARY L 205

LAWRENCE LIVERMORE NTL LAB

PO BOX 808

LIVERMORE

CA 94551

GREG NIMZ

LAWRENCE LIVERMORE NTL LAB

PO BOX 808

LIVERMORE

CA 94551

TECH INF LBRARY DIV

LAWRENCE LIVERMORE NTL LAB

PO BOX 808 L 53

LIVERMORE

CA 94551 